\title{
The Mirrortron Experiment: A Proof of Principle Test for a Method of Generating $\operatorname{AD} 15$ High Transient Potentials 007
}

Scott Roger Douglass

(Ph.D. Thesis)

September 1993

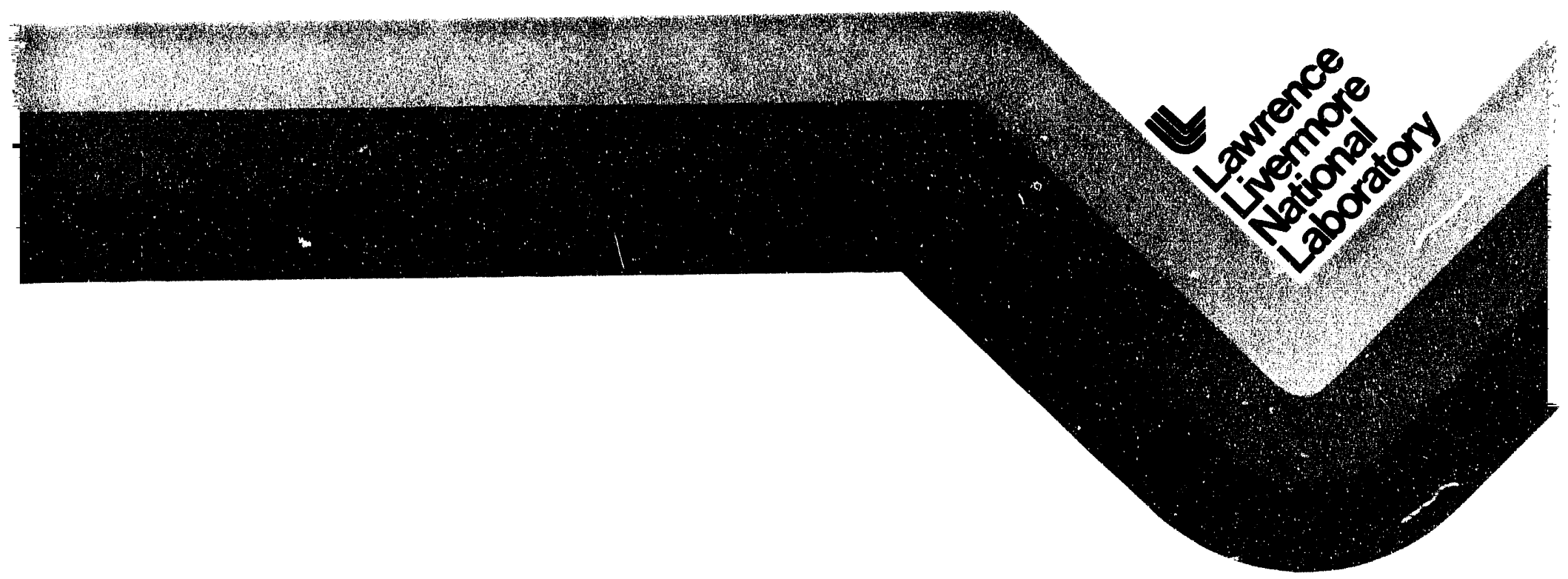




\section{DISCLAIMER}

This document was prepared as an account of work sponsored by an agency of the United States Government. Neither the United States Government nor the University of California nor any of their employees, makes any warranty, express or implied, or assumes any legal liability or responsibility for the accuracy, completeness, or usefulness of any information, apparatus, product, or process disclosed, or represents that its use would not infringe privately owned rights. Reference herein to any specific commercial products, process, or service by trade name, trademark, manufacturer, or otherwise, does not necessarily constitute or imply its endorsement, recommendation, or fa voring by the United States Government or the University of California. The views and opinions of authors expressed herein do not necessarily state or reflect those of the United States Government or the University of California, and shall not be used for advertising or product endorsement purposes.

This report has been reproduced directly from the best available copy.

Available to DOE and DOE contractors from the Omice of Scientific and Technical Information

P.O. Box 62, Oak Ridge, TN 37831

Prices available from (615) 576-8401, FTS 626-8401

Available to the public from the

National Technical Information Service

C.S. Department of Commerce 5285 Port Royal Rd., Springfield, VA 22161

Work performed under the auspices of the U.S. Department of Energy by Lawrence Livermore National Laboratory under Contract W-7405-Eng-48. 


\section{The Mirrortron Experiment: A Proof of Principle Test for a Method of Generating High Transient Potentials}

Scott Roger Douglass

(Ph.D. Thesis)

Manuscript date: September 1993

LAWRENCE LIVERMORE NATIONAL LABORATORY University of California - Livermore, California • 94551 
The Mirrortron Experiment:

A Proof of Principle Test for a Method of Generating High Transient Potentials

By

Scott Roger Douglass

B.S (University of Maryland) 1986

M.S. (University of California, Davis) 1987

DISSERTATION

Submitted in partial satisfaction of the requirements for the degree of

DOCTOR OF PHILOSOPHY

in

Applied Science

in the

GRADUATE DIVISION

of the

UNIVERSITY OF CALIFORNIA

DAVIS

Approved:

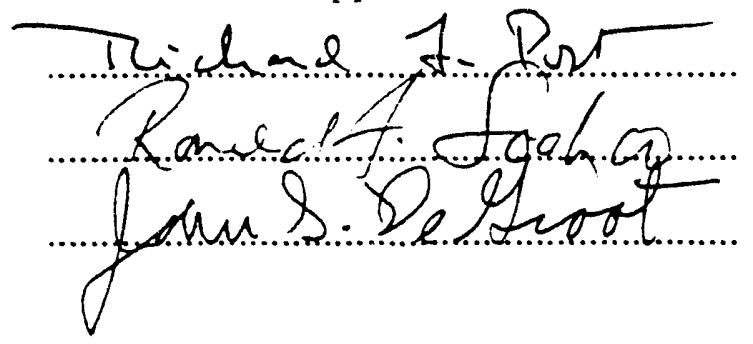

Committee in Charge

1993 
Scott Roger Douglass

September 1993

Applied Science

The Mirrortron Experiment:

A Proof of Principle Test for a Method of Generating High Transient Potentials

\begin{abstract}
The Mirrortron is a concept in which heavy ions are accelerated by a large local transient space potential that is produced in a hot electron plasma. The purpose of this experiment is to begin a proof of principle experiment to investigate the feasibility of producing this space potential and its associated electric field. If a large magnetic field is suddenly generated in a hot electron plasma with a loss-cone distribution, then potentials on the order of the electron temperature are expected. This potential lasts a few tens of nanoseconds.

The investigation begins with a theoretical analysis of this phenomenon giving the space potential as a function of the applied magnetic field. The theory is further extended to cases of reiativistic electron distributions. This is then followed by design work on a mirror confinement system for hot electrons. In this experiment a 50-100 keV electron temperature plasma is created with electron cyclotron resonance heating using two frequencies of realatively low microwave power. The microwaves are coupled to resonant frequencies of the vacuum chamber. The volume averaged plasma density is measured to be in the $10^{9} \mathrm{~cm}^{-3}$ range.

A strap coil and a flat Blumlein transmission line pulse generator were developed to produce a 150 gauss field within $70 \mathrm{~ns}$. The strap coil was placed at the midplane of the mirror field, where the field is 700 gauss. Based on theoretical estimates and computer simulations a $20 \mathrm{kV}$ potential is expected. Measurement of this potential is derived from
\end{abstract}


the modulation of the current of a monoenergetic electron beam after it passes through the high potential region. The variation in the beam energy allows bunching to occur in transit to the decector.

Unexpected limitations were imposed by electrostatic fields that were produced by the feedthroughs to the strap coil. This effect masked the fluctuations from the Mirrortron potential. However, the idea that transient potentials can be measiured by a passing electron beam is demonstrated. 


\section{Acknowledgments}

I would first like to thank Dr. Richard F. Post, who served as my thesis advisor as well as my academic advisor. He has been very helpful in our discussions, and I am grateful for his guidance. The Mirrortron concept was his idea, and I am happy to have been able to extend it to the laboratory. I would also like to thank the other members of my dissertation committee. Dr. John DeGroot was very helpful in getting this manuscript into acceptable shape. Dr. Soohoo's comments helped me to convey a better understanding of the project in the opening chapter.

I would also like to thank the other students who worked briefly on this experiment. The first two were summer students, Kevin Junck and Todd Weisgraber. Then there were two Department of Applied Science students, Mike Lambert and Brian Jones, and a post graduate, Lu Ann Schwager, who joined the project briefly before moving on to other projects. I had many helpful discussions with them, especially Lu Ann's assistance during bench testing of the pulser coil.

I would like to thank the technicians who worked on the experiment at one time or another. Dick Morgret was instrumental in the early stages in getting the Mirrortron running. Thanks to Jeff $V$ an Lue, especially for his design and construction of the tack switch. Thanks to Don Hathaway, the in-house technician, for helping out with the various odd jobs I had for him. Thanks to Bob Leber for his contributing design work on the various Mirrortron parts.

Last, but not least, I would like to thank my family. My wife, Patty Lopez, was encouraging and supportive in the thesis writing stages. Thanks for her efforts in proofreading the manuscript, and her assistance during the last few months of Mirrortron operation. I thank my parents for their love and support through the years; my dad has always been interested in my work, and my mom has always been one of my best friends. May the search for the truth continue... 


\section{Table of Contents}

Chapter I Introduction ............................................................ 1

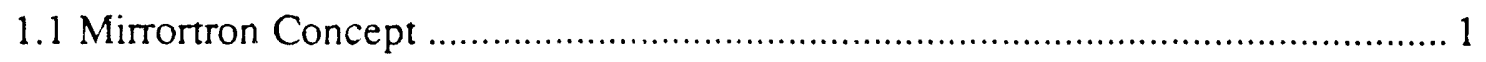

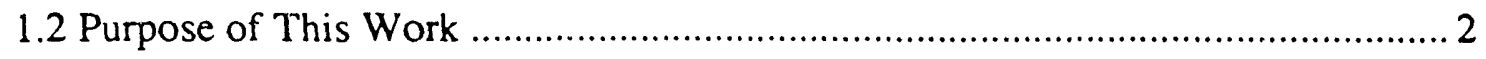

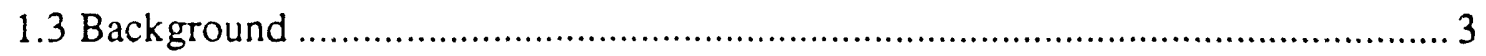

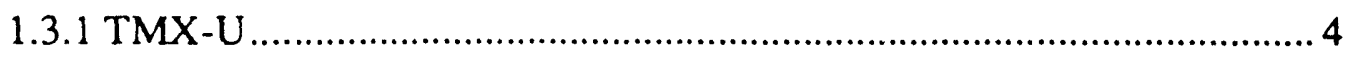

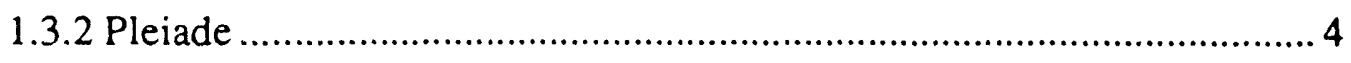

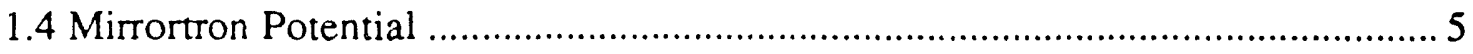

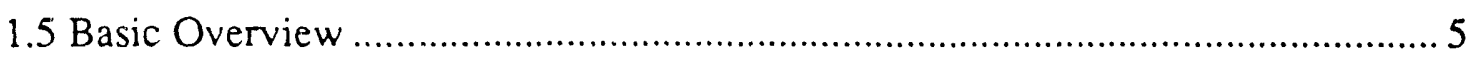

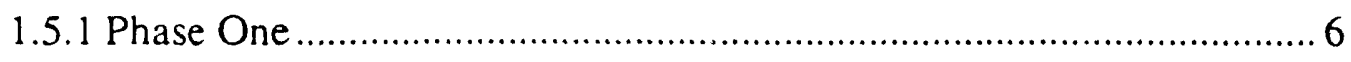

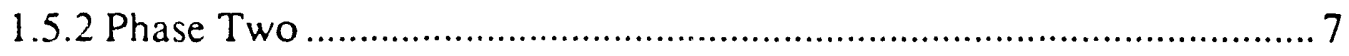

Chapter II Theory of Generating a Transient Potential .......................... 9

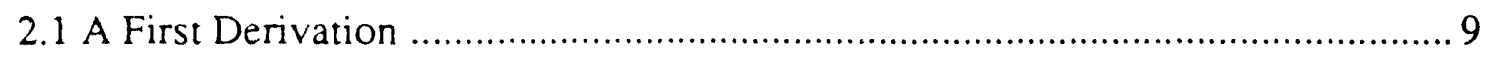

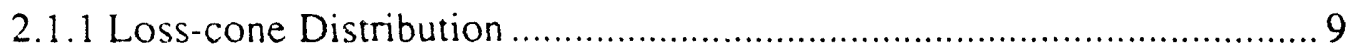

2.1.2 Cold Ions and Hot Electrons ....................................................... 11 


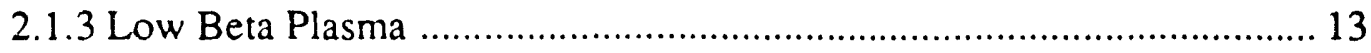

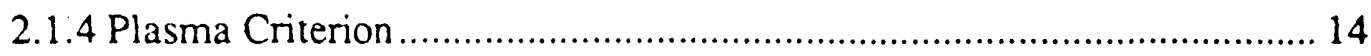

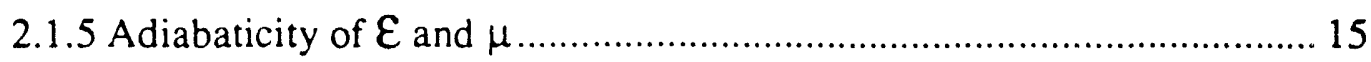

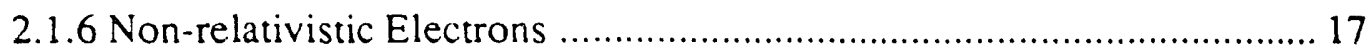

2.1.7 Equation For Transient Potential ....................................................... 17

2.2 A More Detailed Derivation of the Transient Potential .......................................... 23

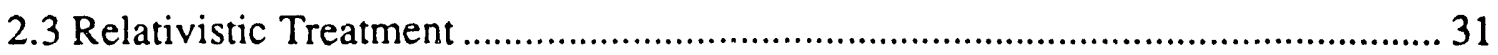

Chapter III Design of the Experiment ................................................. 40

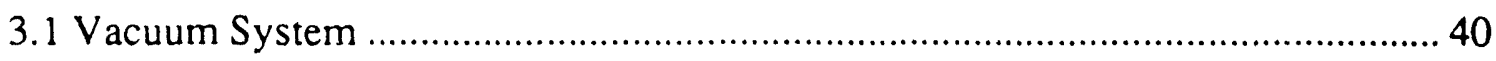

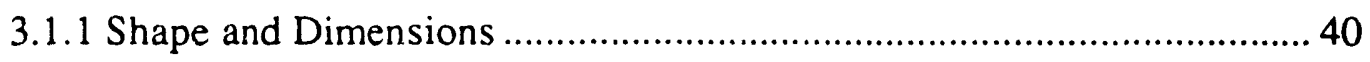

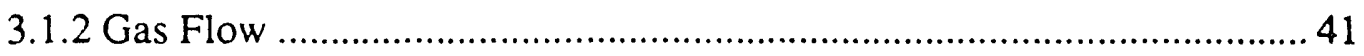

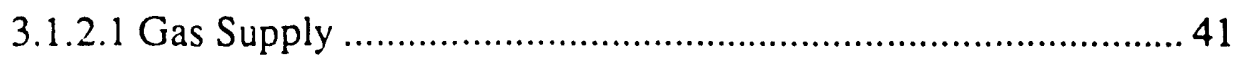

3.1.2.2 Scattering Off Neutrals ........................................................... 43

3.1.2.3 Background Ionization ....................................................... 45

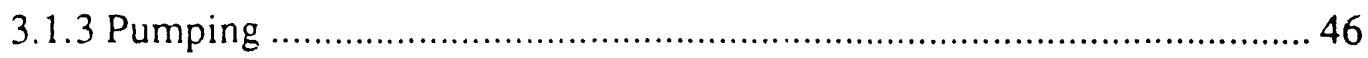

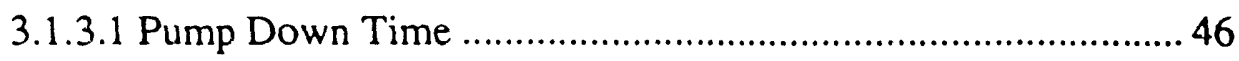

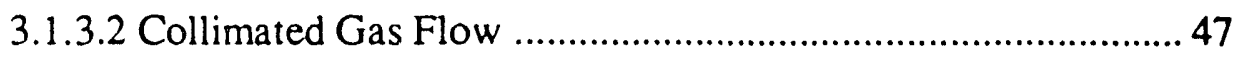

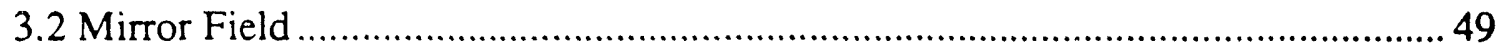

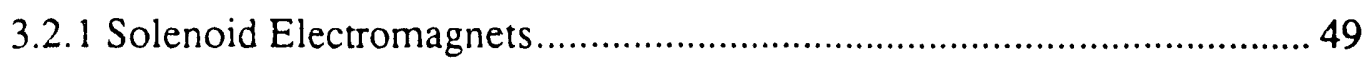

3.2.2 Energy Confinement Calculation ......................................................... 50

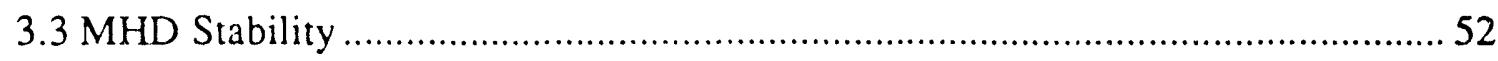

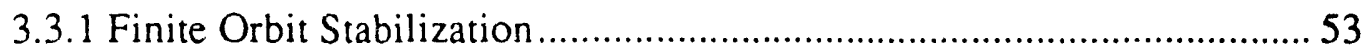

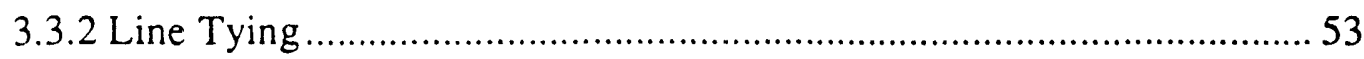

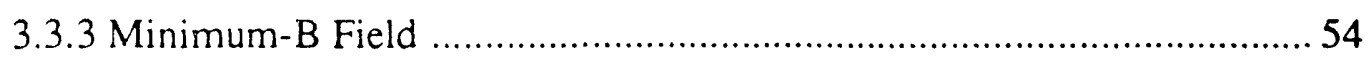

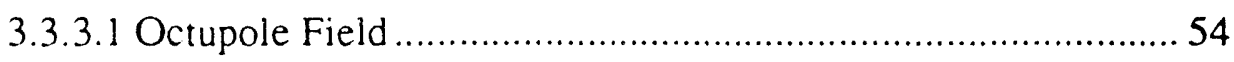

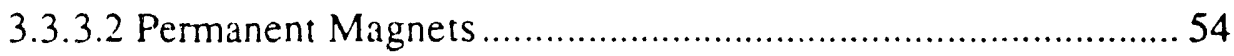


3.3.3.3 Design and Construction of Permanent Magnet Octupole....... 55

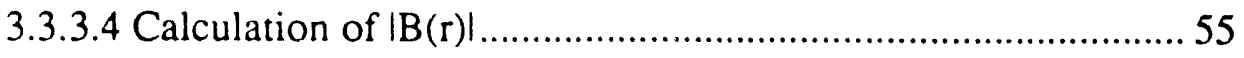

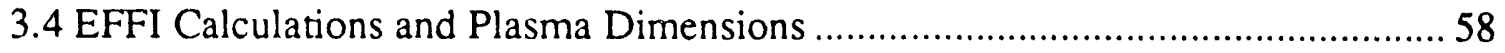

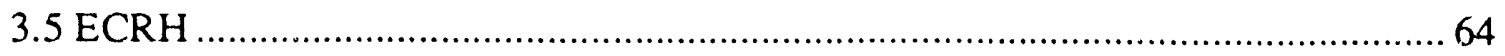

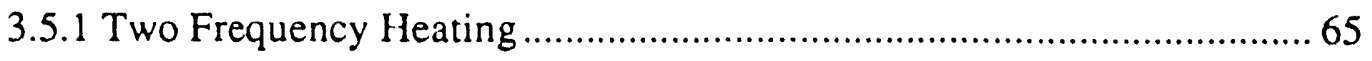

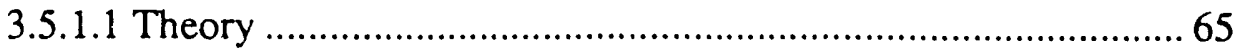

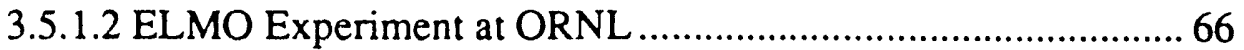

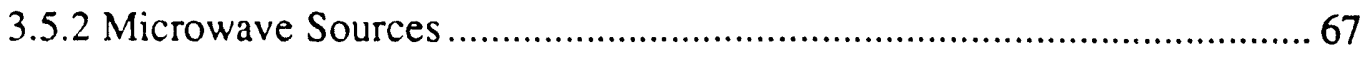

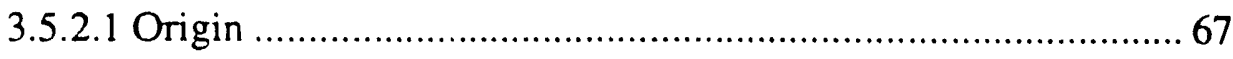

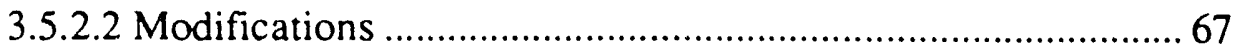

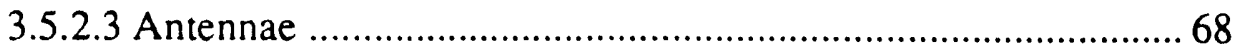

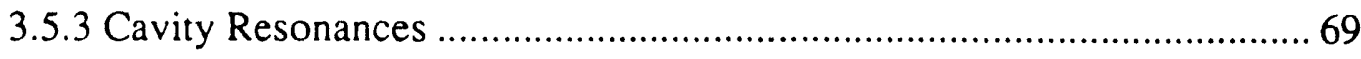

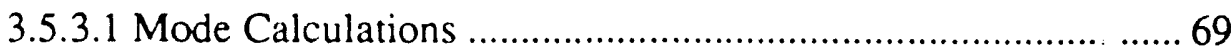

3.5.3.2 Mode Selection ................................................................ 70

3.5.3.3 Measurement of Cavity Modes ................................................ 70

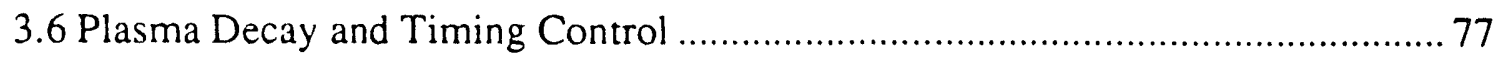

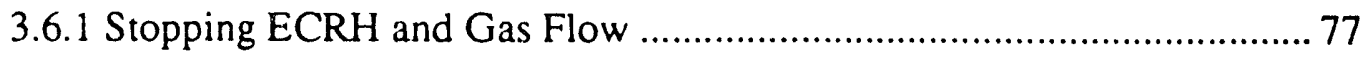

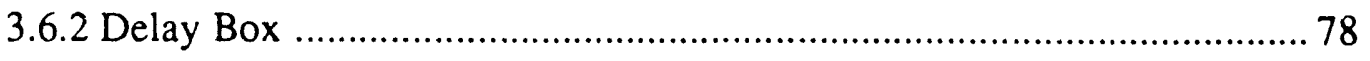

Chapter IV The Hot Electron Plasma ..................................................... 80

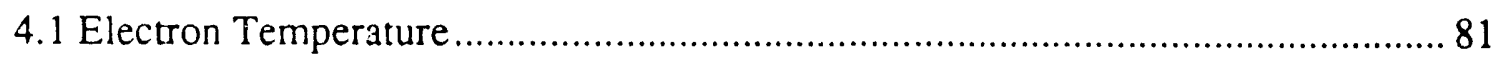

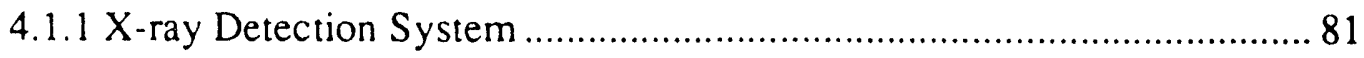

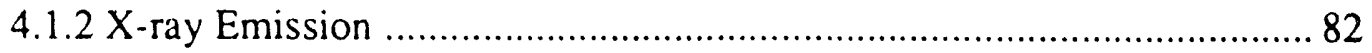

4.1.2.1 Free Electron-Ion Bremsstrahlung …………......................... 82

4.1.2.2 Electron-Wall Bremsstrahlung ………………..................... 83

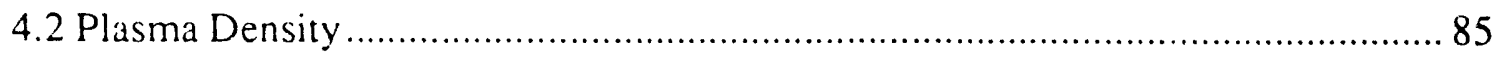

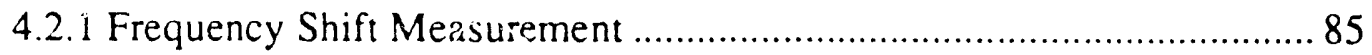




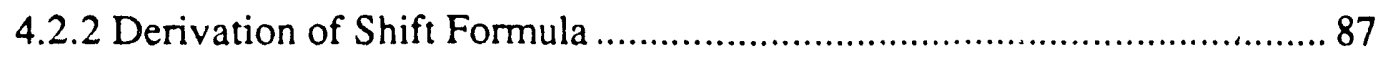

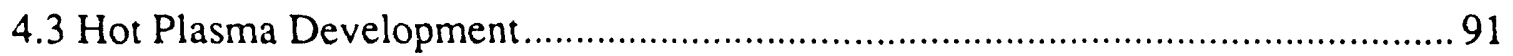

4.3.1 Magnetron Performance ................................................................... 91

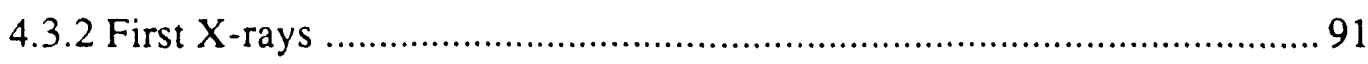

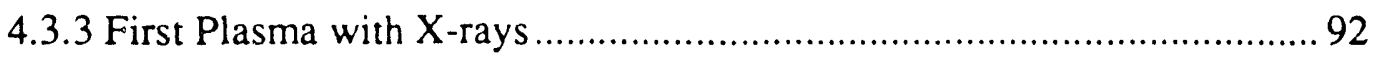

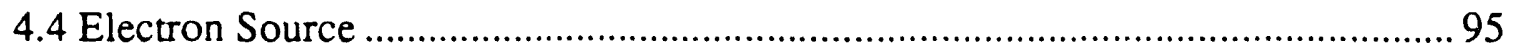

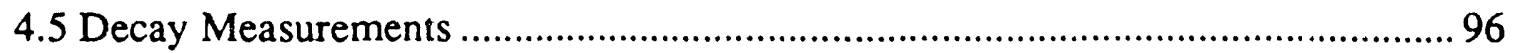

4.5.1 Measurement Using Electron Source ..................................................... 97

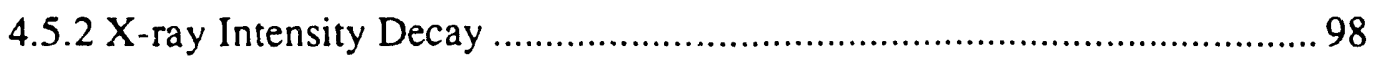

4.5.3 Growth of Oscillator Signal ............................................................... 99

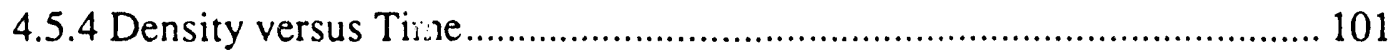

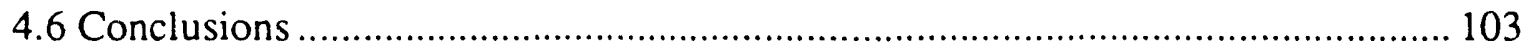

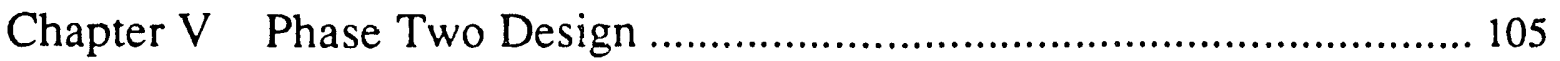

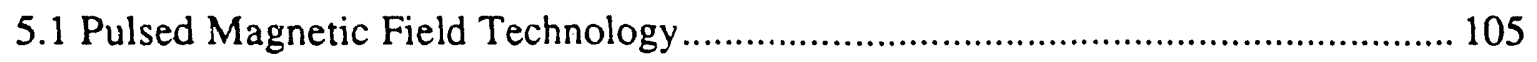

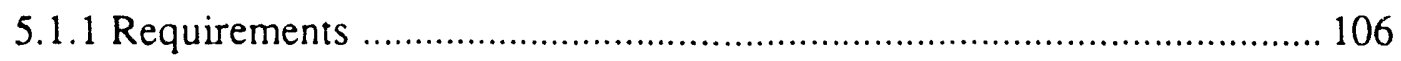

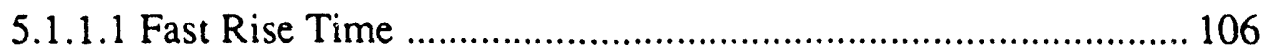

5.1.1.2 Steep B Field Gradient ............................................................ 107

5.1.2 Design of Pulser Coil .......................................................................... 108

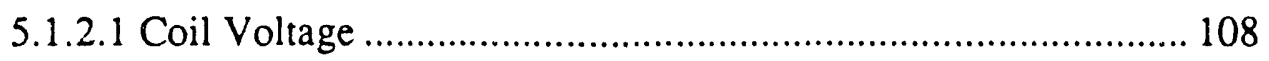

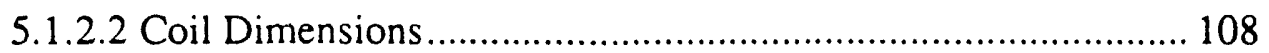

5.1.2.3 Feedthrough Design ............................................................... 112

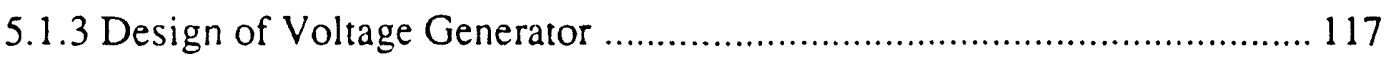

5.1.3.1 Blumlein Transmission Line ................................................. 118

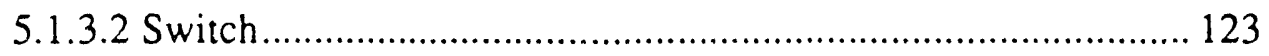

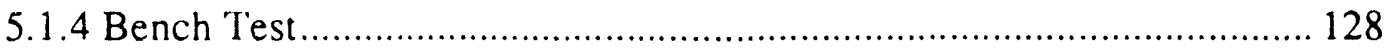

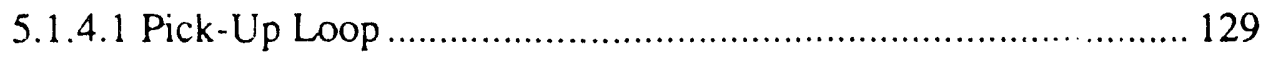


5.2 Electron Beam Diagnostic

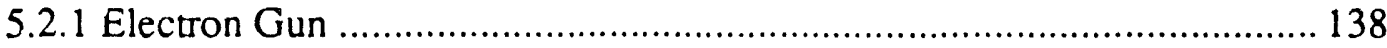

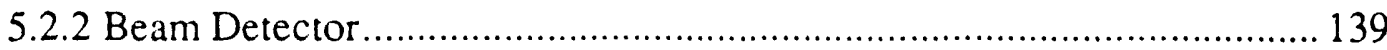

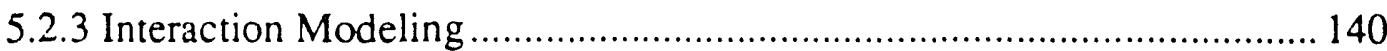

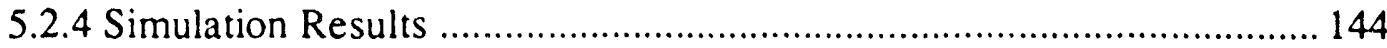

Chapter VI Results of Phase Two ...................................................... 150

6.1 Machine Performance in Second Phase …............................................................ 151

6.1.1 Comparison of X-rays Levels .............................................................. 151

6.2.2 Frequency Shift Measurement Problem................................................ 152

6.2.3 Plasma Particle Collecting ................................................................. 153

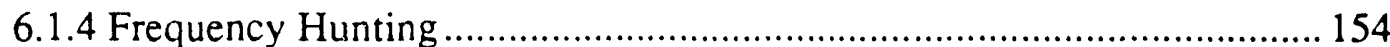

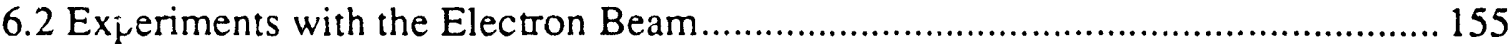

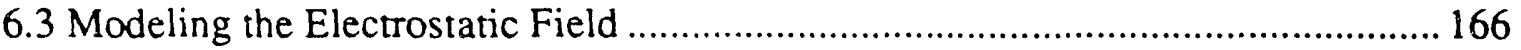

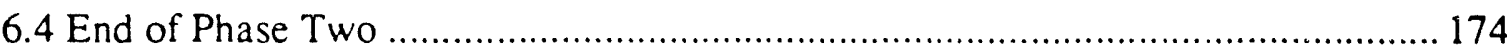

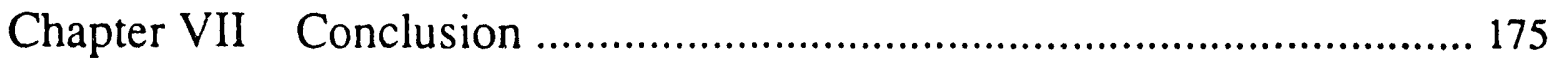

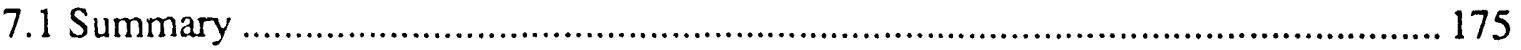

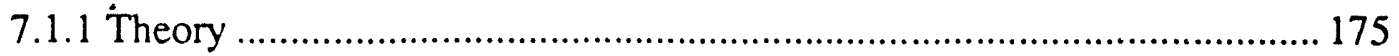

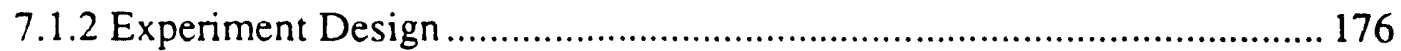

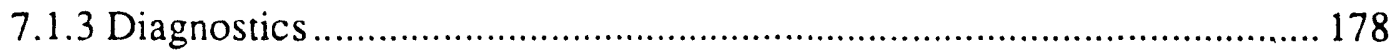

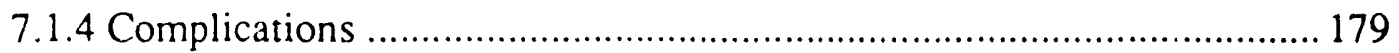

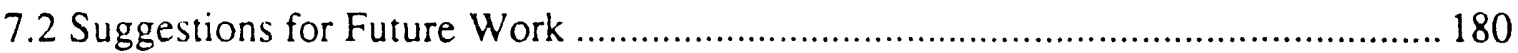

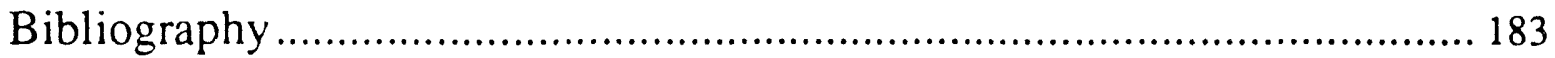




\section{List of Figures}

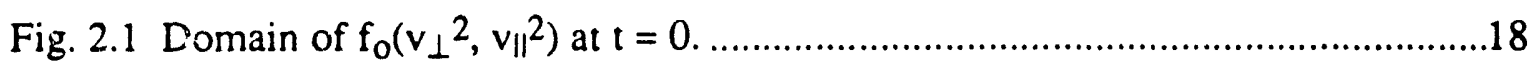

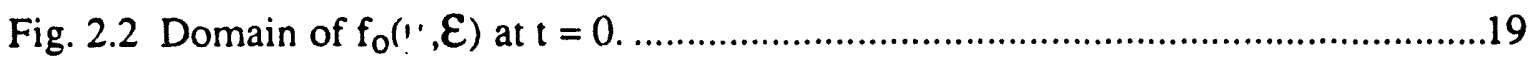

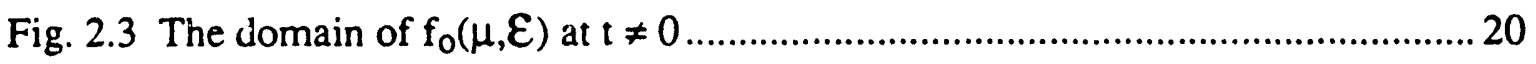

Fig. 2.4 Analytic calculation of the Mirrortron space potential ................................ 24

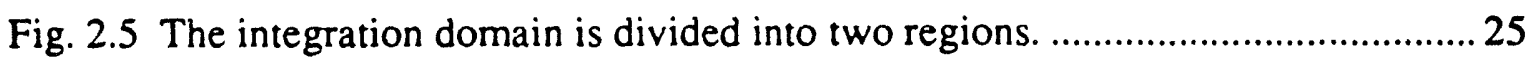

Fig. 2.6 Numerical solution of the space potential using the Gaussian quadrature

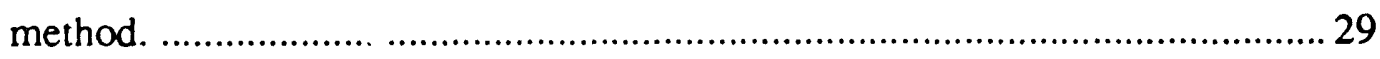

Fig. 2.7 The residual absolute value of eq. 2.35 after the best choice for $\mu_{1}{ }^{\prime}$ has

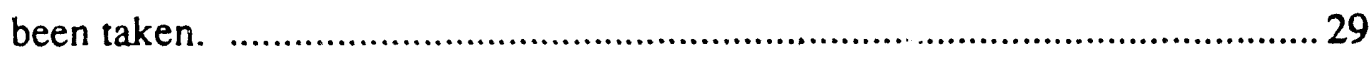

Fig. 2.8 Numerical solution of the space potential using Simpson's rule. .................... 30

Fig. 2.9 The residual absolute value of eq. 2.35 after the best choice for $\mu_{1}^{\prime}$ has

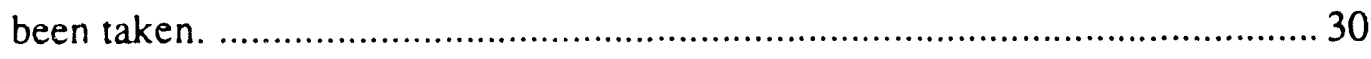

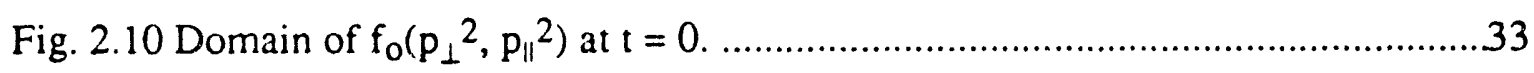

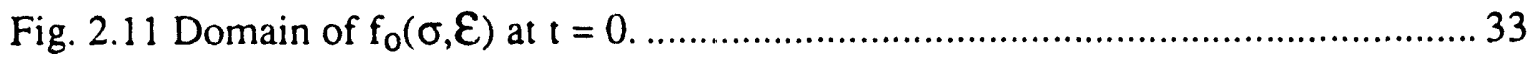

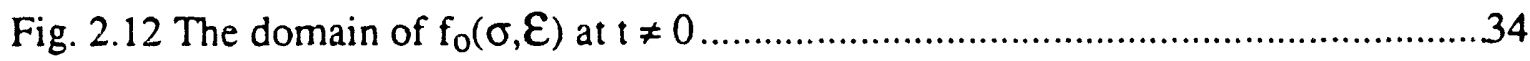


Fig. 2.13 Domain of $f\left(\sigma, p_{\|} c\right)$ at $t \neq 0$.

Fig. 2.14 Numerical relativistic calculation of the space potential for $R_{m}=1.5$........... 38

Fig. 2.15 Numerical solution of the relativistic space potential for $R_{m}=2$ …............. 39

Fig. 2.16 Numerical solution of the relativistic space potential for $R_{m}=1.1$.............. 39

Fig. 3.1 Layout of the Mirtortron Experiment ............................................................ 41

Fig. 3.2 East end of the experiment showing the plenum chamber ............................. 43

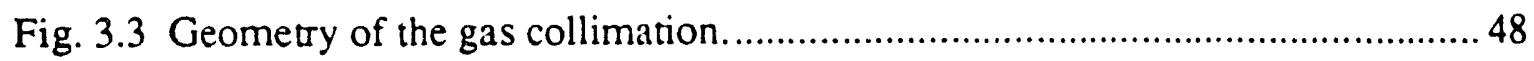

Fig. 3.4 A calculation of the Mirrortron mirror field. ................................................. 51

Fig. 3.5 Orientation of the octupole magnets ...............................................................5 56

Fig. 3.6 Results of a calculation to find the radial position of the minimum in the

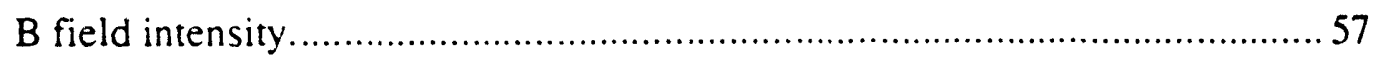

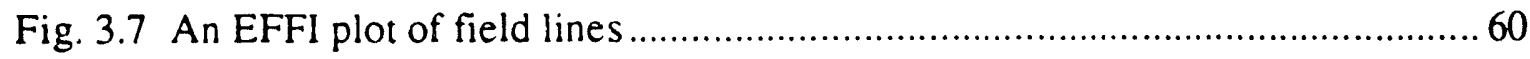

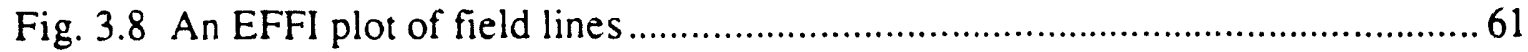

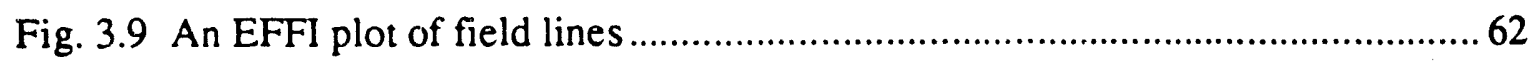

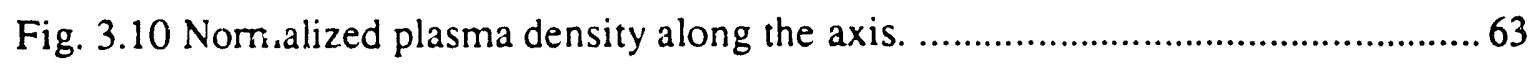

Fig. 3.11 Normalized radial plasma density profile .........................................................64

Fig. 3.12 Antenna port locations on the end flange. .................................................. 72

Fig. 3.13 The first TM resonances of the cavity. ……............................................... 73

Fig. 3.14 A resonance plot of the cavity containing the TM1,1,20 mode......................75

Fig. 3.15 Reflected power of the TM1,1,20 resonance................................................. 76

Fig. 3.16 A resonance plot of the cavity ....................................................................77

Fig. 3.17 A schematic diagram for the delay box....................................................... 79

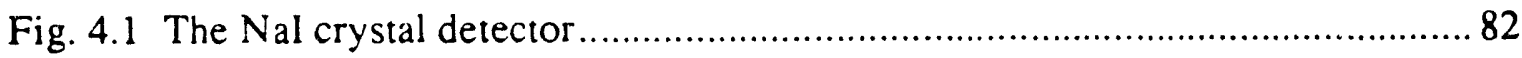

Fig. 4.2 This is the set-up to measure the frequency shift ........................................... 86

Fig. 4.3 The $\mathrm{x}$-ray spectrum measured by the Nal scintillator. ...................................... 94 


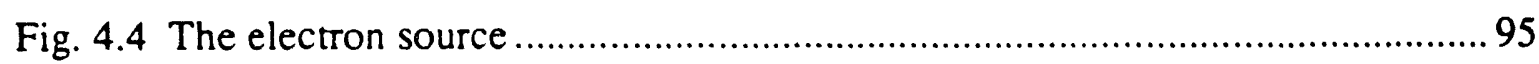

Fig. 4.5 Two voltage-current characteristics measured by the electron source ............. 97

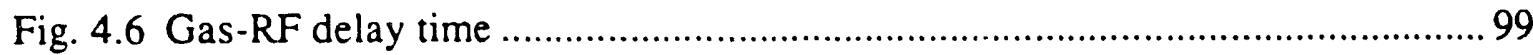

Fig. 4.7 The correlation between the gas-RF delay and the characteristic decay time

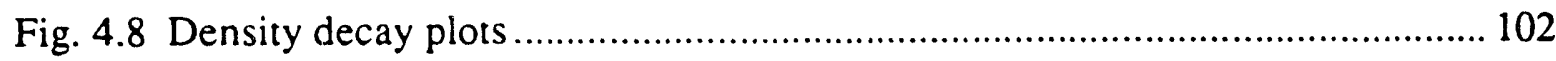

Fig. 5.1 Calculation of the linearly rising magnetic field gradient ............................. 111

Fig. 5.2 A feedthrough port for the pulser coil ........................................................ 112

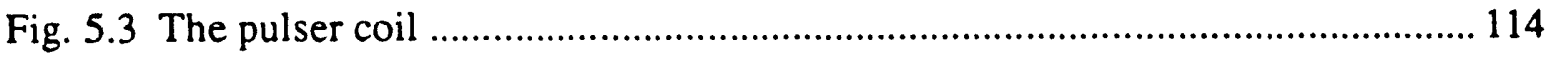

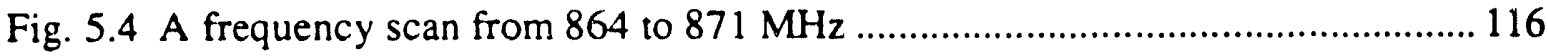

Fig. 5.5 A frequency scan of the first fundamental TM modes .................................... 116

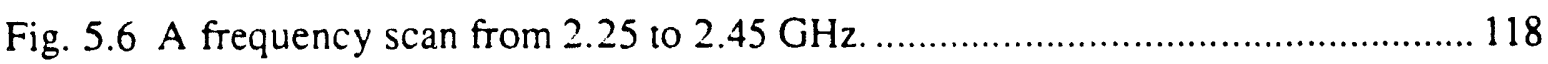

Fig. 5.7 Dimensions of a standard strip transmission line. ......................................... 119

Fig. 5.8 Detail of the cross section of the Blumlein transmission line. ......................... 122

Fig. 5.9 Schematic view of the pulser coil and the Blumlein generator. ....................... 123

Fig. 5.10 The tack switch and the radial transmission line ........................................... 125

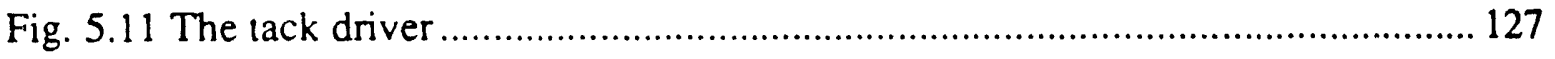

Fig. 5.12 A computer simulation of the voltage front from the radial line ..................... 129

Fig. 5.13 a. Pick-up loop signal ............................................................................. 132

Fig. 5.14 Field rise times for the first switch ....................................................... 134

Fig. $5.15 \mathrm{~B}(\mathrm{t})$ for different radial switch line designs. ................................................. 136

Fig. 5.16 Normalized $\mathrm{B}(\mathrm{t})$ for different charges ..................................................... 137

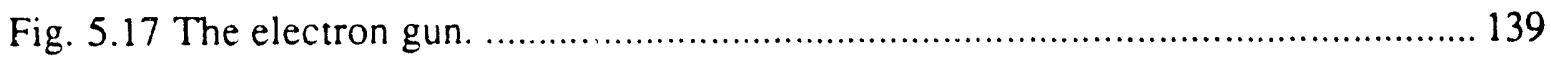

Fig. 5.18 The electron beam detector .................................................................... 140

Fig. 5.19 The theoretical Mirrortron potential .......................................................... 144

Fig. 5.20 Analysis of a $500 \mathrm{eV}$ electron beam …………......................................... 146 


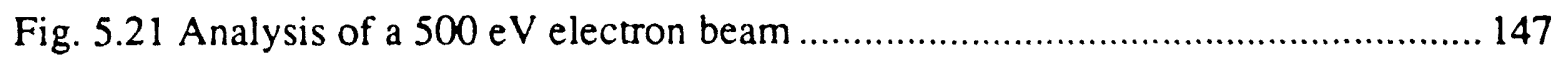

Fig. 5.22 Analysis of a $2 \mathrm{keV}$ electron beam ..................................................... 149

Fig. 6.1 A calibration shot showing fluctuation of the electron beam ........................ 157

Fig. 6.2 The current fluctuation of an electron beam in a plasma .............................. 158

Fig. 6.3 Variation in the current fluctuation as a function of beam energy ................. 160

Fig. 6.4 The effect of the magnetic pulse on the mirror trapped plasma. .................... 161

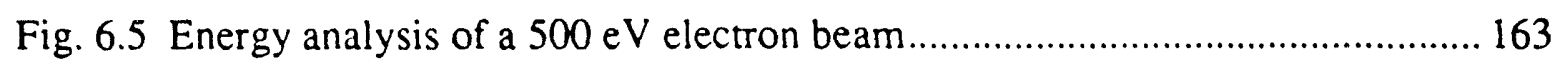

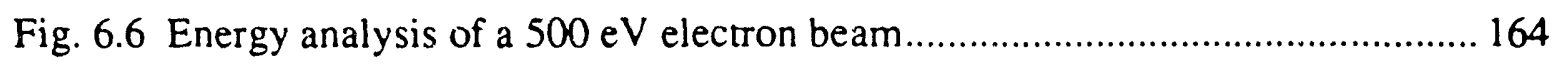

Fig. 6.7 A sketch of the electrostatic potential from the pulser coil ........................... 165

Fig. 6.8 Result of reversing the Blumlein generator polarity ................................. 166

Fig. 6.9 Mathematical functions used to model the pulser coil ................................. 167

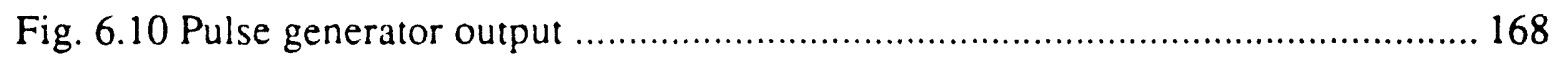

Fig. 6.11 Simulated energy and collected current ................................................ 169

Fig. 6.12 Simulated energy and current signal.................................................. 170

Fig. 6.13 Simulated energy and current signal............................................... 171

Fig. 6.14 Simulation of a $500 \mathrm{keV}$ electron beam ............................................... 173 


\section{List of Tables}

Table 3.1 Characteristic Time for $90^{\circ}$ Coulomb Scattering of Electrons ...................... 44

Table 3.2 Characteristic Time for Scattering Off Neutrals ....................................... 45

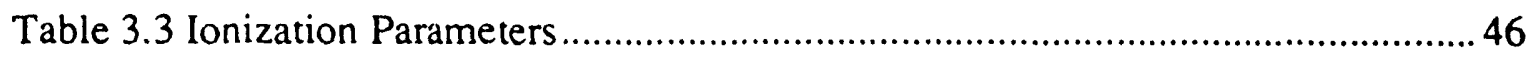

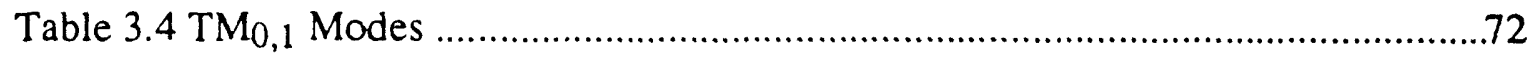

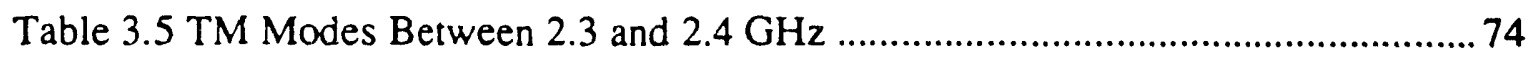

Table 5.1 Effect of Modifications on Resonances ............................................ 117 


\section{Chapter I Introduction}

\subsection{Mirrortron Concept}

The Mirrortron is a name given to an idea for heavy ion acceleration in a magnetic mirror confined plasma. The scheme involves sudden application of a local magnetic field to a hot anisotropic plasma to generate a large localized transient potential. The theory of such a potential indicates that there will be very large electric fields, possibly greater than can be achieved in conventional linear accelerators, if the Mirrortron is designed with the proper parameters. Because of the nature of the acceleration process, this works only for ions, ideally for heavy ions. It is envisioned that the Mirrortron acceleration scheme will be used as a heavy ion driver for inertial confinement fusion (ICF). [1]

In ICF a pellet of fusion fuel is bombarded with high energy, typically in the form of massive, energetic ions. The pellet is compressed and fusion reactions take place. The requirements on the heavy ion driver are that it provide a high current of $\mathrm{GeV}$ ions.

Heavy ion accelerators currently being considered, such as the induction linac, consist of an ion bunch traveling down an evacuated beam pipe, being accelerated as it goes. This 
bunch is accelerated by applying an electric field across a gap between conductors, just as the bunch traverses the gap. Limitations to this scheme result from electrical breakdown across the gap and space charge effects that cause defocusing of the beam. Typical accelerating gradients are a few hundred $\mathrm{keV} / \mathrm{cm}$. [2]

In a Mirrortron accelerator the beam pipe would be filled with a magnetic mirror confined plasma, concentrated near the axis. The accelerating electric field would be contained inside the plasma and be produced by a fast rising magnetic field. This magnetic field is created by a current pulsed strap loop surrounding the plasma. Each strap loop, together wit the plasma, can be thought of as an accelerating element, much like the conductor gap in the vacuum linear accelerator.

The advantages of the Mirrortron for use in ICF are that it is a plasma based accelerator. The space charge of the beam will be reduced and its defocusing effect will be alleviated. As a result, higher ion beam currents will be possible. Also, it is expected that the maximum electric field should exceed that of conventional vacuum linear accelerators, thereby making a more efficient accelerator. Electric fields of about $1 \mathrm{MeV} / \mathrm{cm}$ are theoretically possible. Another advantage is the control over the beam that one still has but would not expect from a plasma accelerator. Because the accelerating field arises from an externally applied magnetic field, the electric fields can be controlled by proper design of the coil used to apply the fast rising magnetic field.

\subsection{Purpose of This Work}

The intent of this work was to begin the investigation of Mirrortron potentials. This means that the first effort should be directed at a proof-of-principle experiment to demonstrate that large electric fields can be generated in a plasma by using pulsed magnetic fields. Details of the acceleration scheme are considerations for later stages of Mirrortron development. No attempt was made to accelerate heavy ions here. 
I began this task to develop a proof-of-principle experiment by first extending the initial theory for a deeper understanding of the physical processes involved in creating the Mirrortron porential. Especially important in this regard is the effect of a relativistic electron distribution.

My next step in this work was to build a mirror confinement device to create a hot anisotropic electron plasma in which to conduct the experiment. This included basic design of the machine and relevant diagnostics. Since this was intended to be a small laboratory experiment, it was important to concentrate on measuring just the fundamental properties of the plasma with the most basic diagnostics. The electron temperature and the plasma density are the two most important parameters of the plasma that were focused on. Also important in the development of the experiment was creating a way to produce a fast rising magnetic field. This includes designing a strap coil and a voltage generator to drive current through it.

I then considered how the transient potential could be detected in the most uncomplicated and easiest fashion. My goal was toward determining the existence of the potential. Characterization of the potential would be for a future experiment. My diagnostic took advantage of the interaction of an electron beam with the potential. The simplest diagnostic would be to measure the current of the beam and then look for fluctuations. Interpretation of the data becomes a bit more complicated when a multichannel energy analysis is forgone. However, I developed a simulation program to help determine what to expect in terms of the current signal.

\subsection{Background}

The origin of the concept for the Mirrortron potential came from other experiments in plasma physics which show that it is possible to generate a large space potential within a plasma. Two examples of such experiments are the thermal barrier used in the TMX-U mirror device and the Pleiade experiment of some years ago. 


\subsubsection{TMX-U}

The TMX-U experiment was an attempt to confine the hot ions of a fusion plasma by creating a large space potential, called the plug potential, in the end cells of a tandem mirror.[3] This was done by exploiting the mirror force resulting from Lorentz forces on a charge moving in a magnetic gradient

$$
F=-\mu \partial B / \partial z
$$

where $\mu$ is the magnetic moment of the electron. This equation is derived in chapter two.

Also important to this effect is the difference in masses of the electrons and ions, which result in different responses to the same force. The mirror force was created locally on a magnetic gradient in a cyclotron resonance zone. At this particular location in the magnetic field, the cyclotron frequency was equal to the frequency of a beam of high power microwaves $(\approx 100 \mathrm{~kW})$ aimed at this spot. This sudden application of electron cyclotron resonance heating $(E C R H)$ increased the electron perpendicular energy on average, and hence the average magnetic moment. This allowed the electrons to experience the mirror force to a much greater degree than the ions; hence the electrons try to leave the region and the local ions behind. Large potential gradients are generated by the requirement for quasineutral density. Quasi-neutrality states that in a sufficiently dense plasma, if the positive and negative charge densities are not exactly equal, then very large electrical restoring forces are created to bring back this equality. Potentials on the order of $1 \mathrm{kV}$ were obtained in the TMX-U. The space potential was maintained essentially for as long as the ECRH was applied.

\subsubsection{Pleiade}

Pleiade was an experiment about 20 years ago in France.[4] A space potential was created in Pleiade in much the same way as in TMX-U. Continuous ECRH was applied to 
a gas stream in an open-ended magnetic gradient. A plasma column formed in the weaker field regions behind the cyclotron resonance zone. The ECRH ionized the gas as well as created a local potential at the resonance zone. There was also a peak in the energy distribution of the ions that left the plasma column, indicating the accelerating possibilities of this potential.

\subsection{Mirrortron Potential}

There is another possible way to generate this potential, since the principles are the same. First, a hot electron plasma is assumed to exist in a uniform magnetic field with mirror fields at either end of the uniform region. The electrons are heated to high temperatures by ECRH. Then the ECRH is stopped and the untrapped particles escape so that the plasma forms a loss-cone distribution in which the electrons have a high average magnetic moment, $\mu$. If a local magnetic field is suddenly applied then the mirror force (eq. 1.1) will appear, strongest on either side of the local magnetic peak. Then a space potential should be produced as in the above mentioned experiments.

Une possible advantage of producing a high transient space potential this way is that it is more efficient because expensive high power microwave generators are not necessary. The energy to produce the localized potential is stored in the electron particle energy. In the Mirrortron this energy can be built up slowly with lower power $(\approx 1 \mathrm{~kW})$ microwaves. The sacrifice comes in having to produce a fast-rising magnetic field. However, the technology involved in producing pulsed magnetic fields is not expected to be more limiting in terms of cost or efficiency than high power microwave generators.

\subsection{Basic Overview}

The experimental effort was divided into two phases. The first part was a check to see that the type of initial conditions necessary prior to pulsing the strap coil can be created. 
This includes generating a stable hot electron plasma with a minimum cold electron component.

The second phase of the experiment involves designing the pulser coil technology to be used to generate the potential, as well as the technology for the electron beam diagnostic used to measure the space potential.

\subsubsection{Phase One}

A mirror plasma is ideal for this application, not only because of the axial access for a heavy ion beam, but, most importantly, because the loss-cone distribution of a mirror confined plasma is required for the generation of the high potential. This will be explained in the theory section.

To start this experiment off we needed to design a mirror confinement system and to decide key dimensions and paramc ters. We chose a solenoidal mirror field with a high order multipole field for stability. A somewhat shallow mirror was desired so we opted for a low mirror ratio of 1.5 with a central field of 700 gauss. It was thought that this field was adequate for trapping high energy electrons. The next important thing to consider was a means of creating high energy electrons.

Electron cyclotron resonance heating is the most obvious means of heating electrons. In the decision between brute force heating and efficiency, we favored efficiency. This meant coupling to a resonant frequency of a high $\mathrm{Q}$ containment vessel. For this purpose a tunable source is very valuable. High power tunable microwave sources are not available so we settled for two 300 watt, tunable, RF sources. Previous work on hot electron plasmas[5] suggested using two RF frequencies to heat the plasma.

Once the design and construction phase was over the first thing I wanted to do was check the operating parameters. The quantities studied were overall stability, volume averaged plasma density, electron temperature, and confinement time. The plasma was 
maintained in steady-state with continuous microwave power. In this mode the density was measured to be on the order of $10^{9} \mathrm{~cm}^{-3}$.

To see if the plasma was being effectively heated, the $\mathrm{x}$-ray spectrum radiating from the plasma was examined. I could only detect high energy $x$ rays of $100 \mathrm{keV}$ or more, hence only the temperature of the high energy electron component could be determined. Radiation of an exponential character $\left(e^{-\varepsilon / k T}\right)$ was observed. From this it was determined that electron temperatures of up to $100 \mathrm{keV}$ were obtained.

Another point to consider is whether the plasma is stable if there is no continuous source for the plasma. The best that can be hoped for is that the plasma will decay according to collisional time scales. This will be particularly long for the hot electron component, on the order of seconds. Decay measurements showed that plasma densities dropped two orders of magnitude very quickly, indicating the quick escape of the cold bulk of plasma. The remaining hot electrons decayed on a time scale of hundreds of milliseconds. This is quicker than anticipated and is probably due to scattering off a relatively high neutral density. Nevertheless, it was still expected that this decay process would provide adequate conditions in which to apply a pulsed magnetic field. There does not seem to be any evidence of a plasma instability that causes an unexpected plasma loss.

\subsubsection{Phase Two}

Once it was determined that I could create adequate plasma conditions, I then concentrated on developing the additional equipment necessary to carry out the experiment. The most important of this equipment is the pulser coil and the electron beam that will be used to measure the potential.

As will be discovered in the next chapter, the requirements for this pulsed coil are stringent. A current rise time of hundreds of amps per nanosecond is necessary. Clearly this coil must be placed inside the containment vessel in order for the desired magnetic field to reach the plasma. One important consideration will be the effect on the current rise time 
as a result of induced eddy currents in the metal containment wall. Another consideration is how the pulser coil will affect the resonant modes of the vacuum cavity, which relates to microwave heating efficiency.

An electron beam is a nonperturbative means of measuring the space potential. It is nonperturbative in the sense that its low charge density and small volume will not affect the physics in the bulk of the mirror trapped in which the transient potential is generated. For this to be effective the electrons must be initially monoenergetic and they must see a net change in the space potential as it traverses the potential region. The electrons then change energy. As the electrons travel to the detector cup, there will be bunching effects resulting in a fluctuating current signal.

Once all the equipment was assembled I devised an experimental procedure to generate a high transient potential. From what is known so far we realize that a hot electron, losscone plasma is needed. So after ECR heating, the plasma must decay long enough for the cold electron bulk to escape, but not so long as to let most of the hot electrons out. At this point the pulser coil must be fired.

The results showed that the electron beam diagnostic will be able to measure the Mirrortron potential when it is produced. The current fluctuations that were at first thought to be produced by the Mirrortron potential, in fact were the result of an artifact of the design of the strap coil that produced the pulsed magnetic field. The vacuum feedthroughs to the coil introduced perturbing electric fields. Electrostatic fields between the feedthroughs interacted with the electron beam and masked plasma based effects.

These fields were external to the plasma but were not screened out, indicating that higher plasma density will be required. High electron temperatures were easily created with our microwave sources, using second harmonic heating. However, greater power will be necessary to increase the electron density. 


\section{Chapter II Theory of Generating a Transient Potential}

Before I begin a description of the experiment and the design work that went into it, I would like to discuss the theory behind the Mirrortron principle. A quantitative description of the transient space potential phenomenon will certainly be necessary to properly design an experiment. The first step is to examine the limits of plasma parameter space in which such a phenomenon can occur. From there a model can be devised for the plasma, then it can be used to derive an equation that describes the behavior of the space charge potential.

\subsection{A First Derivation}

The first derivation will be nonrelativistic. In addition, simplified boundary conditions will be used so that the density distribution integral may be solved analytically.

\subsubsection{Loss-cone Distribution}

First a loss-cone distribution is assumed. From a qualitative understanding of this potential generating effect, we know that it is based on the mirror force: 


$$
F_{z \alpha}=-\mu_{\alpha} \frac{\partial B}{\partial z}
$$

with the magnetic moment $\mu_{\alpha}$

$$
\mu_{\alpha}=\frac{m_{\alpha} v_{\perp}^{2}}{2 B}
$$

where $\alpha$ refers to the charge species. Eq. 2.1 can be derived from conservation principles. For a charged particle traveling along a magnetic gradient $\partial B / \partial z$ with velccity $v_{z}$, no work is actually done and the total energy is conserved. The total energy is, using eq. 2.2

$$
\begin{aligned}
E & =\frac{1}{2} m v_{z}^{2}+\frac{1}{2} m v_{\perp}^{2} \\
& =\frac{1}{2} m v_{z}^{2}+\mu B
\end{aligned}
$$

Conservation of energy means that $\mathrm{d} \varepsilon / \mathrm{dt}=0$. In addition, $\mu$ is an adiabatic constant also, so that $d \mu / d t=0$ as well. This is explained further in section 2.1.5. Applying this to eq. 2.3 gives

$$
\frac{d \mathcal{E}}{\mathrm{dt}}=0=m v_{z} \frac{d v_{z}}{d t}+\mu \frac{d B}{d t}
$$

In the refertince frame of the particle the changing magnetic field is given by $\mathrm{dB} / \mathrm{dt}=\partial \mathrm{B} / \partial \mathrm{t}$ $+v_{z} \partial B / \partial z$. The mirror field is constant so $\partial B / \partial t=0$. Hence eq. 2.4 becomes

$$
\begin{gathered}
0=v_{z}\left(m \frac{d v_{z}}{d t}\right)+\mu v_{z} \frac{\partial B}{\partial z} \\
0=F_{z}+\mu \frac{\partial B}{\partial z}
\end{gathered}
$$


Eq. 2.5 is equivalent to eq. 2.1 , the mirror force.

The force acts most strongly on electrons with a large magnetic moment, $\mu$. For a high average force on the electron distribution, the plasma should have a loss-cone distribution. This is because the electrons that would be present in the loss-cone phase space do not respond well to $F_{z}$. The smaller the mirror ratio is, the stronger the average force will be.

\subsubsection{Cold Ions and Hot Electrons}

Second, a large difference in electron and ion temperatures is assurned. To produce a local positive space potential in a plasma, some of the electrons must be forced out of the local region. This means the miror force must preferentially move the electrons. Some advantage is gained due to the greater inertia of the ions. However, to achieve a greater average force on the electrons, the condition $\left\langle\mu_{e}\right\rangle »\left\langle\mu_{\mathrm{i}}\right\rangle$ must be satisfied. This calls for electrons with high perpendicular energy, and the ions should be as cold as possible, i.e., ionization energies. These conditions are typical of ECRH plasmas.

Limits on the cold ion assumption are determined by the length of the magnetic pulse. The mirror force is not charge dependent, so that both species move in the same direction. If the pulse is too long the ions will begin to move into a charge balance with the electrons, and the potential will begin to disappear. Hence, the transient nature of the potential is apparent. An upper limit to the pulse length can be estimated based on the time it takes an ion to move. The maximum magnetic pulse length should be much less than the time it takes an ion to move an appreciable distance. If we assume motionless ions, then this simplifits the calculation by only considering the electron behavior during the magnetic pulse.

Consider the time it takes for the mirror force to move a cold ion across a distance $z$ of the magnetic gradient. Take the extent of the magnetic gradient to be $\mathrm{L}$ and the gradient to be $\Delta B / L$. Assume the force to be constant over the distance $z$. 


$$
F=\mu \frac{\Delta B}{L}
$$

Here $\mu=W_{\perp} / B_{0}$, and $B_{0}$ is the background field. Position as a function of time using $F=$ ma can now easily be found

$$
z=\frac{1}{2} \frac{F}{m_{i}} t^{2}
$$

and the time to move a distance $\mathrm{z}$ is

$$
t=\sqrt{\frac{2 m_{i} z L}{\mu \Delta B}}
$$

Since the potential magnitude will depend on $\Delta \mathrm{B}$, this equation shows that the greater the applied magnetic field, the less time the transient potential can last. Consider an example magnetic field of $\Delta B=B_{0} / 2$, and typical values of $W_{\perp}=20 \mathrm{eV}$ for cold hydrogen ions, and $L=10 \mathrm{~cm}$. For fixed ions the maximum ion motion should be a distance small compared to $\mathrm{L}$. Hence, if $z=1 \mathrm{~mm}$ then it likes $460 \mathrm{~ns}$ for the ion to move this distance. The potential might last this long, but it assumes that the transient magnetic field is already applied. Therefore, the field should rise to $B_{0} / 2$ in a time short compared to $460 \mathrm{~ns}$. The field rise time should be a few hundred gauss in just a few tens of nanoseconds.

Now if the conditions are right an electric field will result from ions lagging in response to the magnetic force. But how will the ions respond to this electric field? The time it tikes the ion lo move acruss the electric field due to the force from the electric field can be determined in the same way as for the magnetic force by using eq. 2.8 , but by replacing $\mu \Delta B$ with the peak potential energy, le $\mid \Phi_{0}$. We will soon discover that the potential will be proportional to the electron temperature. Hence, for $\mathrm{kT}_{\mathrm{e}}=100 \mathrm{keV}, \Phi_{0}=$ $2.5 \mathrm{kV}$ (see fig. 5.19 ). Again, if $\mathrm{L}$ is $10 \mathrm{~cm}$, then the time to move across $1 \mathrm{~mm}$ is $\mathrm{t}=9 \mathrm{~ns}$ 
for hydrogen ions. This may strain the fixed ion assumption, but the time has been estimated on the short side. Nevertheless, a heavy ion species such as argon could be used, and it would slow the ion motion by a factor of six.

\subsubsection{Low Beta Plasma}

A third assumption is a low beta plasma, i.e., the particle pressure, $n k T_{e}$, is small in comparison to the magnetic pressure, $\mathrm{B}^{2} / 2 \mu_{0}$. Here we are concerned with a magnetic pulse being applied to a plasma, and one characteristic of plasmas is that they are very conductive. The possibility exists that the plasma might prevent this fast rising pulse from penetrating the plasma to the axis. For a low beta plasma this should not present a problem, but it does put an upper limit on the plasma density.

The plasma density will play a key role in magnetic shielding. To examine the magnetic shielding consider a plasma column with a boundary at $r=a$. The boundary condition as applied to the tangential $\mathbf{H}$ field at the surface of the medium is

$$
\mathbf{n} \times\left(\mathbf{H}_{2}-\mathbf{H}_{1}\right)=\mathbf{J}
$$

where $\mathbf{n}$ is the unit surface normal pointed outward from the plasma, and $\mathbf{H}_{2}$ is outside, and $\mathbf{H}_{1}$ is inside the plasma boundary, and $\mathbf{J}$ is surface current density (amps $/ \mathrm{m}$ ) on the boundary. If the plasma conductivity were infinite, then the field inside the plasma, i.e., $\mathbf{H}_{1}$, would be zero, and the magnitude of $\mathbf{J}$ would be equal to the magnitude of $\mathbf{H}_{2}$, that is if the plasma can supply the high current density needed. The volume current density is determined by

$$
\mathbf{j}=\mathrm{en}_{\mathrm{e}} \mathbf{v}
$$


The current density (amps $/ \mathrm{m}^{2}$ ) is electric charge multiplied by electron density multiplied by average electron velocity. The ion contribution is negligible. To obtain a surface current $j$ is multiplied by some effective surface layer of thickness $d$. Consider the maximum J possible (by letting the effective thickness be the entire radius of the column, $d=a$, and letting the electrons travel at maximum speed, speed of light, $v=c$ ); this will give the maximum field, $\mathbf{H}_{2}$, that the plasma can effectively screen out.

$$
B_{2 \max }=\frac{H_{2 \max }}{\mu_{0}}=\frac{\text { eca }}{\mu_{\mathrm{o}}} n_{\mathrm{e}}
$$

From this equation it can be calculated that an electron density of $10^{9} \mathrm{~cm}^{-3}$ would correspond to a magnetic field of about 60 gauss given that the plasma radius is $10 \mathrm{~cm}$. If a few hundred gauss will be applied, this density seems appropriate. Perhaps $10^{10} \mathrm{~cm}^{-3}$ might even be possible given the extreme way in which $\mathrm{J}$ was calculated.

\subsubsection{Plasma Criterion}

The fourth assumption is the basic plasma criterion. The transient potential effect is dependent on the collective behavior of the plasma. Therefore, it is important that the plasma satisfy the criteria for a plasma. This states that the Debye length of the plasma is substantially less than the dimensions of the plasma

$$
L>\lambda_{D}=\sqrt{\frac{\varepsilon_{\mathrm{o}} k T_{e}}{n \mathrm{e}^{2}}}
$$

Most importantly, eq. 2.12 states that the processes in the bulk of the plasma are not influenced by the potential of the surrounding containment vessel. If the grounded wall were not shielded out, then the high potential on axis would be reduced significantly. 
To ensure that the plasma is unaffected by the wall, a lower limit must be set on the electron density. The radius of the vacuum vessel is $13 \mathrm{~cm}$, hence, $\mathrm{L}=13 \mathrm{~cm}$. Since it is expected that the electron temperature will be about $50-100 \mathrm{keV}$, then for an electron density of $10^{9} \mathrm{~cm}^{-3}, \lambda_{\mathrm{D}}$ will be $5-7 \mathrm{~cm}$. A density of $10^{8} \mathrm{~cm}^{-3}$ gives Debye lengths of 17 $23 \mathrm{~cm}$. Clearly a plasma of this density would be too thin. In this experiment there is a narrow range of densities that is acceptable, but in general a bigger vacuum tank is a simple solution to this problem.

If the plasma criterion 2.9 is satisfied, then it is possible to make the statement that the plasma is quasi-neutral. This basically means that the electron and ion densities are equal, but not so equal that the electromagnetic effects caused by these free charges are reduced to zero. The Mirrortron potential depends on a separation of charge, but it can be easily looked at from another point of view. There is an electric field associated with the Mirrortron potential that is acting on the electrons. This electric force is equal and opposite to the mirror force applied to the plasma. Hence there is a balance of forces that allows the electrons to remain in the same local concentrations as before, equal to that of the ions. This fact will be invoked later in the derivation of the Mirrortron potential.

\subsubsection{Adiabaticity of $\varepsilon$ and $\mu$}

Another element of the theory is that the magnetic moment of the electrons, $\mu_{e}$, as well as their total energy, $\mathcal{E}$, are constants of their motion. This is not hard to believe when the electrons are just bouncing back and forth in the mirror field, but we will also assume that it is true during the rapid rise of the locally pulsed magnetic field. Doubts arise since Faraday induction associated with a fast rising magnetic field can create an azimuthal electric field that can increase the perpendicular energy of the electrons. However, this energy gain will be small compared to its total perpendicular energy if the increase in the magnetic field, $\delta \mathrm{B}$, during one cyclotron period is much less than the magnetic field itself. That is, if 


$$
\frac{\delta \mathrm{B}}{\mathrm{B}} \ll 1
$$

For example the midplane field, $\mathrm{B}_{0}$, might be 600 gauss and so the gyration period would be $0.6 \mathrm{~ns}$. It is expected that there will be a pulsed field rise time of $300 \mathrm{~g}$ in $60 \mathrm{~ns}$, in which case, the increase in the magnetic field over a gyration period is $\delta \mathrm{B}=3 \mathrm{~g}$. The fraction in eq. 2.13 is then 0.005 which satisfies the criteria.

A general proof of adiabatic invariants shows that $\mu$ is indeed very nearly a constant, since any variances are exponentially small.

$$
\frac{\Delta \mu}{\mu} \sim e^{-B / \delta B}
$$

The dependence of $\mathrm{W}_{\perp}$ on $\delta \mathrm{B} / \mathrm{B}$ is not quite so small.

$$
\frac{\delta \mathrm{W}_{\perp}}{\mathrm{W}_{\perp}}=\frac{\delta \mathrm{B}}{\mathrm{B}}
$$

Most electrons will move into lower field regions as the local field rises, either under the influence of the mirror force or by their own momentum. Hence, the $\delta \mathrm{B}$ seen by the electron will be smaller, if not zero, than what it would be for an electron fixed in space. Therefore, $\delta W=0$. Other electrons will experience some energy gain. These electrons are at the midplane, where there is no magnetic gradient, and have no parallel velocity. However, this energy gain will be small, and the fraction of these electrons of the whole population will be small. Thus, it can be approximately stated that the average energy of the electrons is constant. 


\subsubsection{Non-relativistic Electrons}

The last assumption to be made is probably the biggest one. Assume a non-relativistic plasma. In our experiment electron temperatures are 50-100 keV, which is borderline relativistic. However, it is possible to form $\mathrm{MeV}$ plasmas, and for such cases nonrelativistic treatments are not possible. The theory will be much easier to treat for the nonrelativistic case, and it is, of course, a starting point. Once this is done we will have a better idea of how to begin a relativistic treatment.

\subsubsection{Equation For Transient Potential}

We are now ready to begin the derivation of the transient potential equation. Since this effect will occur in a mirror plasma, the first thing to do is to arrive at a model for a losscone distribution. Such a model is given in eq. 2.16 [6].

$$
\begin{aligned}
f_{0} & =A \sqrt{v_{\perp}^{2}-K^{2} v_{\|}^{2} v_{\perp}^{2} \exp \left(-v_{\perp}^{2} / v_{\perp 0}^{2}\right)} & v_{\perp}^{2}>K^{2} v_{\|}^{2} \\
& =0 & v_{\perp}^{2}<K^{2} v_{\|}^{2}
\end{aligned}
$$

with $\mathrm{K}$ and $\mathrm{v}_{\perp 0}$ defined by

$$
\mathrm{K}^{2}=\frac{1}{\mathrm{R}_{\mathrm{m}}-1} \quad \frac{1}{2} \mathrm{mv}_{\perp 0}^{2}=\mathrm{kT}_{\perp}
$$

This distribution has the feature that it is continuous at the loss-cone boundary.

Now if eq. 2.16 is expressed in terms of adiabatic invariants $\mu$ and $\varepsilon$, then we have

$$
\begin{aligned}
f_{o} & =A \sqrt{R_{m} \mu B_{o}-\varepsilon} \mu \exp \left(-2 \mu B_{o} / v_{\perp o}^{2}\right) & & \varepsilon<R_{m} \mu B_{o} \\
& =0 & & \varepsilon>R_{m} \mu B_{o}
\end{aligned}
$$

Here $\mu$ and $\varepsilon$ are mass normalized for convenience, and are generally given by 


$$
\mu=\frac{1}{2} \frac{v_{\perp}^{2}}{B} \quad \varepsilon=\frac{1}{2}\left(v_{\perp}^{2}+v_{\|}^{2}\right)+\phi
$$

The mass normalized potential energy is given by

$$
\phi=\frac{\mathrm{e} \Phi}{\mathrm{m}}
$$

and $\Phi$ is the potential. Other constants in eq. 2.18 have been absorbed into the normal factor $\mathrm{A}$.

Now eq. 2.16 describes the electron distribution at $t=0$, just before the pulsed magnetic field is applied. Eq. 2.18 is for $\mathrm{t}=0$ as well, i.e., when $\mathrm{B}=\mathrm{B}_{\mathrm{o}}$ and $\phi=0$. But the advantage to expressing $f_{o}$ in terms of invariants is that $f_{o}(\mu, \mathcal{E})$ is time independent and so it is also valid even when $B \neq B_{0}$ and $\phi \neq 0$.

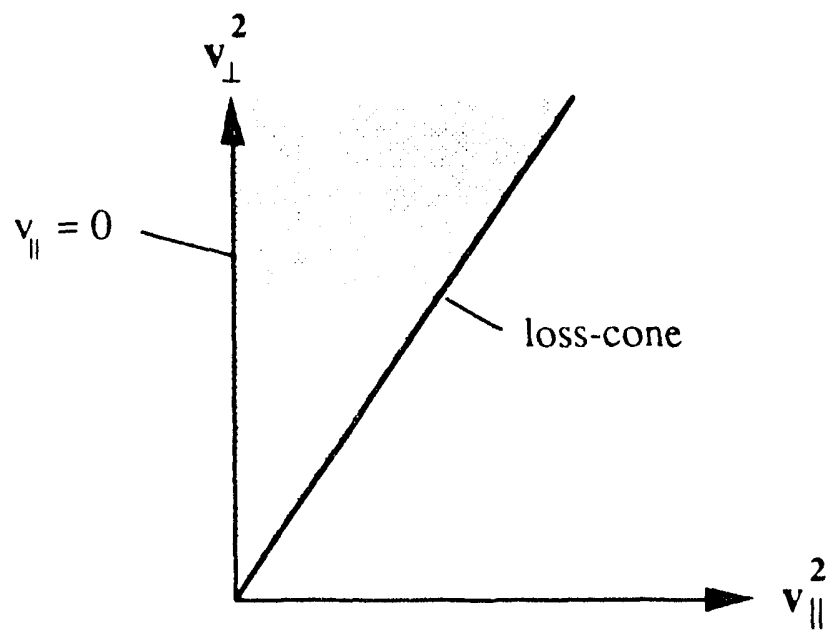

Fig. 2.1 Domain of $f_{o}\left(v_{\perp}^{2}, v_{\|}^{2}\right)$ at $t=0$. 


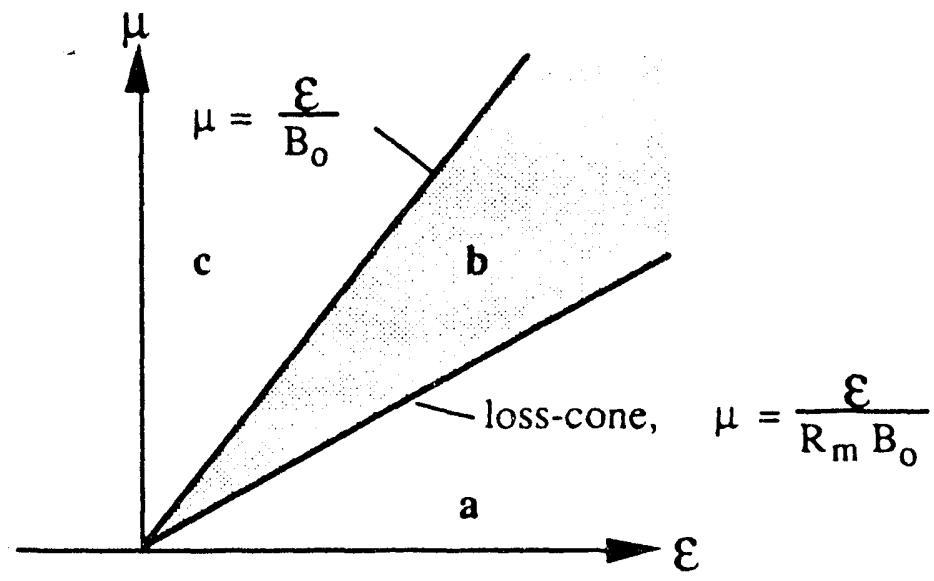

Fig. 2.2 Domain of $f_{o}(\mu, \mathcal{E})$ at $t=0$.

Now integrate over invariant space to find an expression for the electron density that involves $B$ and $\phi$. These terms enter into the equation by means of the boundary conditions and the Jacobian of the transformation. The loss-cone boundary in eq. 2.16 is defined by $v_{\perp}^{2}=K^{2} v_{\|}^{2}$, which translates to $\mu=\varepsilon /\left(R_{m} B_{0}\right)$. However, the other boundary in velocity space must not be forgotten: $v_{\|}^{2}=0$. This boundary becomes $\mu=\varepsilon / B_{0}$. Figs. 2.1-2 demonstrate this transformation.

Regions $\mathbf{a}$ and $\mathbf{b}$ in fig. 2.2 are easily understood as the areas of phase space for untrapped and trapped electrons respectively. Region $\mathbf{c}$ is forbidden space because it describes electrons with perpendicular energy greater than its total energy. For any time after $\mathrm{t}=0$ these boundary conditions can be expressed generally in terms of $B$ and $\phi$.

$$
\begin{array}{cc}
\mathrm{R}_{\mathrm{m}} \mu \mathrm{B}=\mathcal{E}-\phi & \text { loss-cone } \\
\mu \mathrm{B}=\varepsilon-\phi & \mathrm{v}_{\| 1}^{2}=0
\end{array}
$$




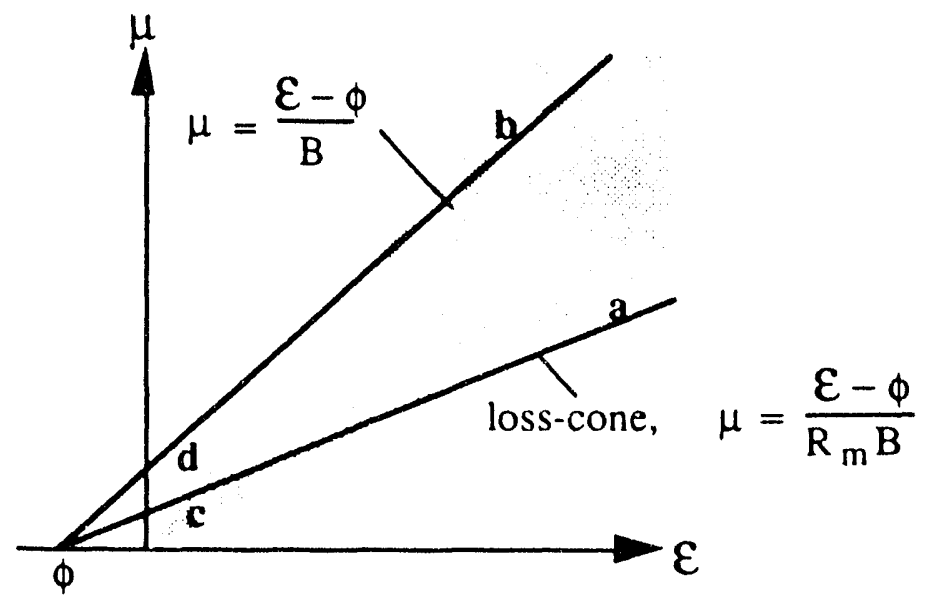

Fig. 2.3 The domain of $f_{o}(\mu, \mathcal{E})$ at $t \neq 0$ is between the solid lines. The shaded area is where $f_{0}$ is still defined.

Fig. 2.3 shows the boundaries at later times. The boundaries have moved and have created new regions of phase space with interesting physical interpretations. Region a corresponds to a section of velocity space that describes barely trapped electrons with a minimum pitch angle. Because of the increasing magnetic field the pitch angle increases, leaving the area near the loss-cone vacant. Region $\mathbf{b}$ describes electrons with near zero parallel velocity for which the increasing $B$ field demanded a corresponding increase in perpendicular velocity greater than the electron's total energy would allow. Most of these electrons leave the local area, and a few will actually gain energy, thus disobeying our assumption of constant energy. In this second case we ignore the contribution of these electrons to the density integral for reasons stated previously. Region $\mathbf{c}$ includes low energy electrons that have crossed over the loss-cone boundary and have become untrapped, magnetically speaking. However, they are still held in by the existence of a positive potential.

Region d describes low energy electrons that have gained parallel kinetic energy because their potential energy has decreased due to the presence of the space potential. Because of this gain the electrons (shaded area) appear shifted to the right with respect to 
the boundary. This is only true of the electrons that move in and out of the potential region in a time fast compared with the potential rise time. The parallel kinetic energy gain is then equivalent to the magnitude of the potential. Slower electrons are more like electrons in a metal box as the potential of the box rises. There are no electric fields inside the box to act on the electron and change its velocity. These electrons do not travel across the entire potential gradient as it rises, hence, its parallel kinetic energy is not equivalent to the potential. For these electrons the conservation of total energy is not true.

Consider a 50-100 keV plasma and lower energy electrons of $10 \mathrm{keV}$ or less. If their parallel energy is $1 \mathrm{keV}$ (near the $\mathrm{v}_{\|}=0$ boundary) then the transit time across a $10 \mathrm{~cm}$ potential scale length is about $5 \mathrm{~ns}$. This is reasonably less than a potential rise time of tens of nanoseconds, hence we can say energy is conserved for these electrons. The percentage of electrons less than $10 \mathrm{keV}$ in a hot electron plasma should be small, therefore, they will not contribute much to the integral. Hence, region $\mathbf{d}$ will be described as vacant.

From this discussion we can see what the boundaries of the integration region should be. The right hand boundary should be the unchanged loss-cone boundary, $\varepsilon=R_{m} \mu B_{0}$, since the distribution is not defined beyond it. Also the low energy electrons of region $\mathbf{c}$ will be included that otherwise would not be if the loss-cone boundary of fig 2.3 were chosen. The left hand boundary should be the unchanged boundary, $\varepsilon=\mu \mathrm{B}_{0}$, up to where region $b$ begins, since $f_{0}$ is not defined to the left of it. Where area $b$ starts, the boundary should be $\mathcal{E}-\phi=\mu \mathrm{B}$. So that $\mathrm{f}_{\mathrm{o}}$ may be integrated analytically, it is claimed that region $\mathrm{d}$ is small and has a negligible effect on the integral. So now $\mathrm{d}$ may be included and the left boundary condition in fig. 2.3 may be used for the entire left side.

Electron density comes from integrating eq. 2.16 over velocity space

$$
n_{e}=\int_{-\infty}^{\infty} d v_{\|} \int_{K v_{\|}}^{\infty} v_{\perp} d v_{\perp} f_{o}\left(v_{\perp}, v_{\|}\right)
$$


Conversion to invariant space gives

$$
\mathrm{n}_{\mathrm{e}}=\mathrm{B} \int_{0}^{\infty} \mathrm{d} \mu \int_{\mu \mathrm{B}+\phi}^{\mathrm{R}_{\mathrm{m}}^{\mu \mathrm{B}_{o}}} \mathrm{~d} \boldsymbol{\varepsilon} \frac{\mathrm{f}_{\mathrm{o}}(\mu, \varepsilon)}{\sqrt{\varepsilon-\mu \mathrm{B}-\phi}}
$$

This can be done analytically to give the following result

$$
n_{e}=A B\left[2\left(R_{m} B_{o}-B\right)-\left[\frac{e \Phi B_{0}}{2 k T_{e}}\right]\right]
$$

At this point the quasi-neutrality principle comes in. The electron density is equal to the ion density at all times. Since the ion distribution is constant over the time scale of interest, $n_{e}$ is constant and it is equivalent to the electron density at $t=0$. Hence we have

$$
\mathrm{n}_{\mathrm{e}}(\mathrm{B}, \Phi)=\mathrm{n}_{\mathrm{e}}\left(\mathrm{B}=\mathrm{B}_{\mathrm{o}}, \Phi=0\right)=2 \mathrm{AB}_{0}^{2}\left(\mathrm{R}_{\mathrm{m}}-1\right)
$$

Eqs. 2.24 and 2.25 can be combined and rearranged to give an expression for $\Phi$. This is the equation first derived by Post.[5]

$$
\Phi=\frac{2 k T_{e}}{|e|}\left[\left[\frac{B}{B_{0}}-\frac{B_{0}}{B}\right]-R_{m}\left[1-\frac{B_{0}}{B}\right]\right]
$$

Analyzing eq. 2.26 can give insight into the behavior of this phenomenon. The first thing that is noticed is the potential's dependence on the electron temperature. $\Phi$ is linearly proportional to $\mathrm{T}_{\mathrm{e}}$. Hot electrons mean high potentials, so the importance of electron heating prior to pulsing a magnetic field is obvious. 
Fig. 2.4 shows the dependence of $\Phi$ on $\mathrm{B} / \mathrm{B}_{\mathrm{o}}$ for three different mirror ratios. When the applied field has approximately doubled, then the potential will be one to two times the equivalent electron temperature. As expected, the potential rises faster for a smaller mirror ratio. The point is that the average applied mirror force is greater for plasmas with higher concentrations of electrons with large pitch angles. The only difficulty in using a low mirror ratio is the problem of maintaining a high enough plasma density such that the plasma criteria is satisfied. For $R_{m} \geq 2.0$ an initial increase in $B$ does not cause any increase in $\Phi$. Hence, $R_{m}=1.5$ seems like a practical value.

One of the most important things about the functional form of $\Phi$ that really makes the Mirrortion practical is that it only depends on the local value of $B$. This means that surfaces of constant B are equivalent to equipotential surfaces. The magnetic field from a simple single turm loop can create equipotentials with a focusing character. Precise control and beam shaping can be accomplished by careful design of the pulser coil. This is a desirable feature in any particle accelerator.

\subsection{A More Detailed Derivation of the Transient Potential}

At this point it would be interesting to see what kind of results we get when region $\mathbf{d}$ is taken into account. What should be done is to not include this area in the domain of integration. It seems that the best way to do this is to break the domain up into two areas as shown in fig. 2.5. The dividing line is at $\mu_{1}$, which is the intersection of the left boundary lines $\mu B_{0}=\varepsilon($ for $t=0)$ and $\mu \mathrm{B}=\mathcal{E}-\phi($ for $t \neq 0)$. The result is

$$
\mu_{1}=\frac{-\phi}{B-B_{0}}
$$




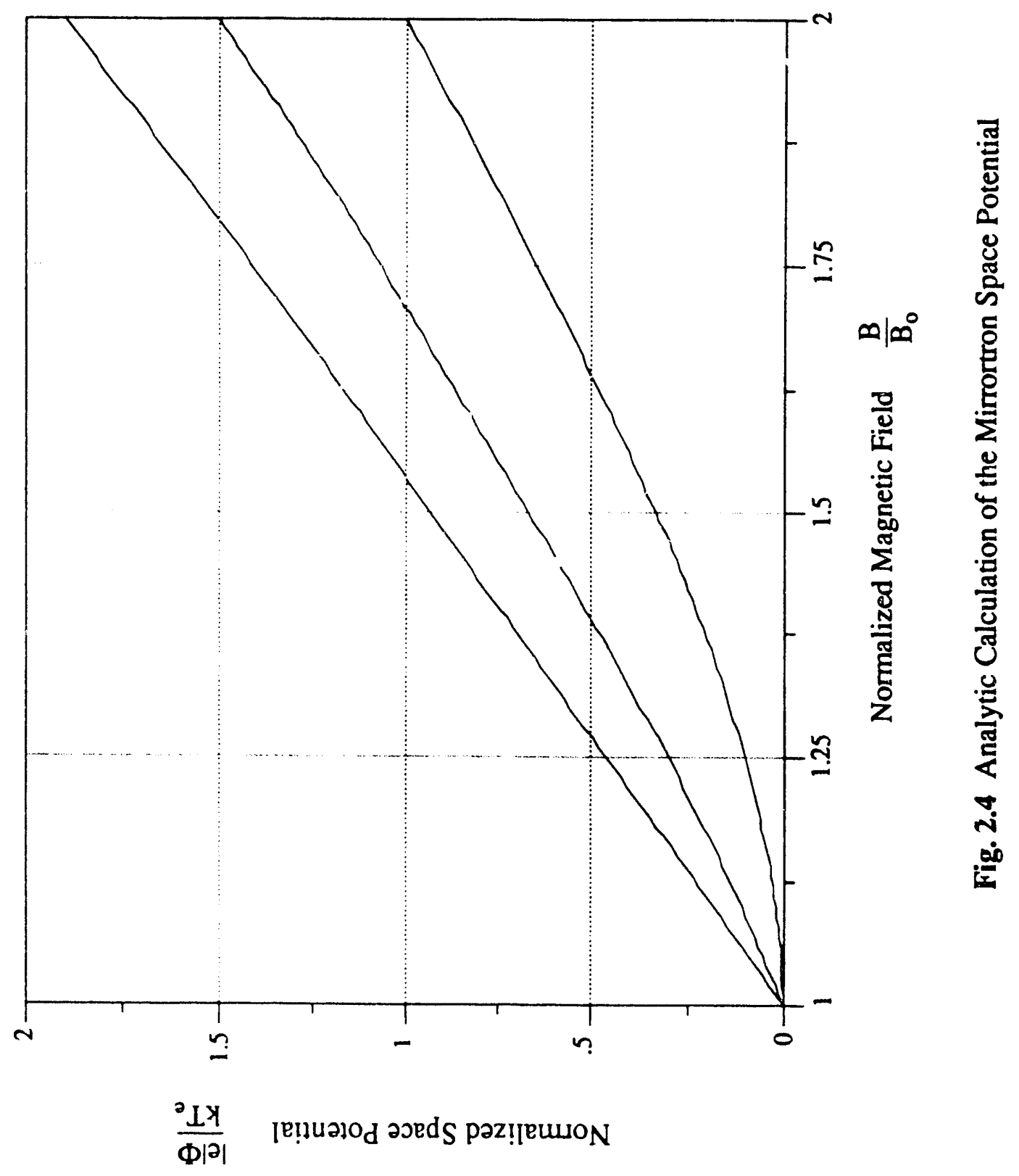




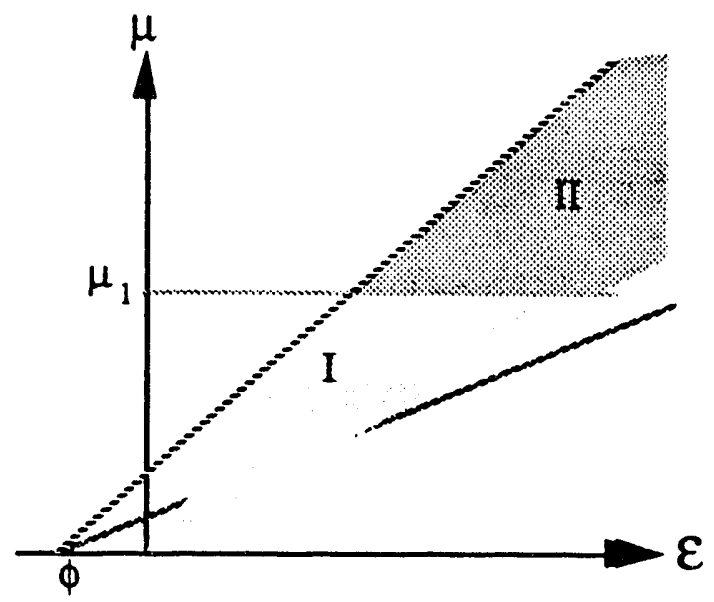

Fig. 2.5 The integration domain is divided into two regions.

The integral for electron density now becomes

$$
\begin{aligned}
& \mathrm{n}_{\mathrm{e}}=\mathrm{I}_{1}+\mathrm{I}_{2} \\
& I_{1}=B \int_{0}^{\mu_{1}} d \mu \int_{\mu B_{0}}^{R_{m}^{\mu B_{o}}} d \varepsilon \frac{f_{0}(\mu, \varepsilon)}{\sqrt{\varepsilon-\mu B-\phi}} \\
& I_{2}=B \quad \int_{\mu_{1}}^{\infty} d \mu \int_{\mu B+\phi}^{R_{m}^{\mu} B_{0}} d \varepsilon \frac{f_{o}(\mu, \mathcal{E})}{\sqrt{\varepsilon-\mu B-\phi}}
\end{aligned}
$$

$I_{1}$ covers region I in fig 2.5 , and cannot be done analytically. $I_{2}$ is over region II, and can be done analytically but results in a transient equation for $\phi$. With the following definitions 


$$
\mu_{0}=\frac{v_{\perp 0}^{2}}{2 B_{0}}=\frac{k T_{e}}{\mu B_{0}} \quad \mu_{1}^{\prime}=\frac{\mu_{1}}{\mu_{0}}=\frac{l e \mid \Phi}{k T_{\perp}\left(\frac{B}{B_{0}}-1\right)}
$$

the expression for $I_{2}$ can be given as

$$
I_{2}=A B B_{0} \frac{\pi}{2} \mu_{o}^{3} e^{-\mu_{1}}\left\{\left(R_{m}-\frac{B}{B_{0}}\right)\left[\mu_{1}^{2}+2 \mu_{1}^{\prime}+2\right]+\left(\frac{B}{B_{0}}-1\right)\left[\mu_{1}{ }^{2}+\mu_{1}^{\prime}\right]\right\}
$$

Concentrating on $I_{1}$ we see that the integral over energy, $\varepsilon$, can be done, but this leaves an integrand for the $\mu$ integral that is too complicated to do analytically.

$$
\begin{gathered}
I_{1}=A B B_{0} \mu_{0}^{3} \int_{0}^{\mu_{1}^{\prime}} d \mu^{\prime} \mu^{\prime} e^{-\mu^{\prime}}\left\{g\left(\mu^{\prime}\right) \sin ^{-1} \sqrt{\frac{\left(R_{m}-1\right) \mu^{\prime}}{g\left(\mu^{\prime}\right)}}-h\left(\mu^{\prime}\right)\right\} \\
g\left(\mu^{\prime}\right)=\left(R_{m}-\frac{B}{B_{0}}\right) \mu^{\prime}+\left(\frac{B}{B_{0}}-1\right) \mu_{1}^{\prime} \\
h\left(\mu^{\prime}\right)=\sqrt{\left(R_{m}-1\right) \mu^{\prime}\left(\frac{B}{B_{0}}-1\right)\left(\mu_{1}^{\prime}-\mu^{\prime}\right)}
\end{gathered}
$$

The next thing to do is to apply the quasi-neutrality constraint to the problem to get a working equation. Letting $\phi=0$ and $B=B_{0}, I_{1}$ and $I_{2}$ merge to become one integral that gives the following result.

$$
\mathrm{n}_{\mathrm{e}}=A B_{0}^{2} \pi \mu_{0}^{3}\left(R_{m}-1\right)
$$

Combining it all together we get 


$$
\begin{gathered}
\int_{0}^{\mu_{1}^{\prime}} d \mu^{\prime} \mu^{\prime} e^{-\mu^{\prime}}\left\{g\left(\mu^{\prime}\right) \sin ^{-1} \sqrt{\frac{\left(R_{m}-1\right) \mu^{\prime}}{g\left(\mu^{\prime}\right)}-h^{\prime}\left(\mu^{\prime}\right)}\right\}+ \\
\frac{\pi}{2} e^{-\mu_{1}^{\prime}}\left\{\left(R_{m}-\frac{B}{B_{0}}\right)\left[\mu_{1}{ }^{2}+2 \mu_{1}{ }^{\prime}+2\right]+\left(\frac{B}{B_{0}}-1\right)\left[\mu_{1}{ }^{2}+\mu_{1}^{\prime}\right]\right\}-\frac{B}{B_{0}}\left(R_{m}-1\right) \pi=0
\end{gathered}
$$

To solve this problem I used a procedure whereby trial values of $\mu_{1}{ }^{\prime}$ were chosen for a given $\mathrm{B} / \mathrm{B}_{0}$. I kept track of the right hand side of eq. 2.35 and chose the $\mu_{1}{ }^{\prime}$ that gave the smallest value. I started at $\mu_{1}{ }^{\prime}=0$ for $\mathrm{B} / \mathrm{B}_{0}=1$. Then $\mathrm{B} / \mathrm{B}_{0}$ was increased by intervals of .01 . Trial values of $\mu_{1}{ }^{\prime}$ also varied by .01 . The quantity lel$\Phi / k T_{\perp}$ is calculated from $\mu_{1}{ }^{\prime}$ and $\mathrm{B} / \mathrm{B}_{\mathrm{o}}$ using eq. 2.31 .

Once $\mathrm{B} / \mathrm{B}_{0}$ and a trial value of $\mu_{1}{ }^{\prime}$ were chosen, $\mathrm{I}_{1}$ could then be numerically integrated. I tried two different methods. The first was Gaussian quadrature. In this method we have a problem integral in the form of

$$
\int_{0}^{\mu_{1}^{\prime}} d \mu^{\prime} f\left(\mu^{\prime}\right) w\left(\mu^{\prime}\right)
$$

and we want to approximate this by a finite sum

$$
\sum_{i=1}^{n} A_{i} f\left(\mu_{i}\right)
$$

where $\mu_{i}$ 's are the zeroes of an nth-degree polynomial with respect to the weighting function and on an interval specific to that polynomial. The $A_{j}$ 's are coefficients that depend on the type of polynomial used and the order $n$. This method essentially approximates $f(\mu$ ') with an orthogonal polynomial of order $2 n-1$ and this method is exact if $f\left(\mu^{\prime}\right)$ is a 
polynomial of order $\leq 2 n-1$. Our question is do we use coefficients and zeroes determined from Legendre or Laguerre polynomials?

The integral is on a finite interval and is easily transformed to a Legendre interval, $(-1,1)$. However, the exponential factor $\mathrm{e}^{-\mu^{\prime}}$ is a perfect Laguerre weighting function and is eliminated from the function to be approximated by the Gauss-Laguerre method.

Unfortunately the integrand must be defined on a $(0, \infty)$ domain. Exponentials are not easy to approximate by polynomials, but since only the values of $\mu^{\prime}$ between zero and $\mu_{1}{ }^{\prime}$ are of concern, it will be approximated this way together with the rest of the integrand. In the numerical integration 21 points were used, hence, simulating the integrand with a 41 st order polynomial.

The results of this computation are presented in fig 2.6. To get an idea of the accuracy of these results, I plotted the absolute value of the minimum value of the right hand side of eq. 2.35 as a function of $B / B_{o}$ in fig. 2.7. When this error value oscillates it indicates that eq. 2.35 is solved exactly; elsewhere, a real value for $\mu_{1}^{\prime}$ can not be found that gives an exact solution. For example, when $R_{m}=2$, eq. 2.35 is solved for all values of $B / B_{0}$, but for $R_{m}=1.1$ there is no solution for any value of $B / B_{0}$. I suspect that the problem might be the accuracy of the evaluation of $I_{l}$.

As a secorid independent approach to the problem I decided to integrate $I_{1}$ using Simpson's rule. It is not as elegant as the previous method but it is easy to use as many points as necessary to get the value of $I_{1}$ to converge. Surprisingly it takes only 50 to 100 points. Figs. 2.8 and 2.9 contain the results of this calculation and its error.

In both cases the potential produced is much higher than the analytic solution for a given applied magnetic field, as shown in fig. 2.4. This is because the additional cold electrons of region $\mathbf{d}$ that reduce the potential, were not considered. They are more attracted by the electric field than repelled by the magnetic field. However, it is possible that energy is not conserved for a non-negligible number of electrons and that region $\mathbf{d}$ will have some electrons in it. The curves in fig 2.6 and 2.8 should be considered an upper limit. 


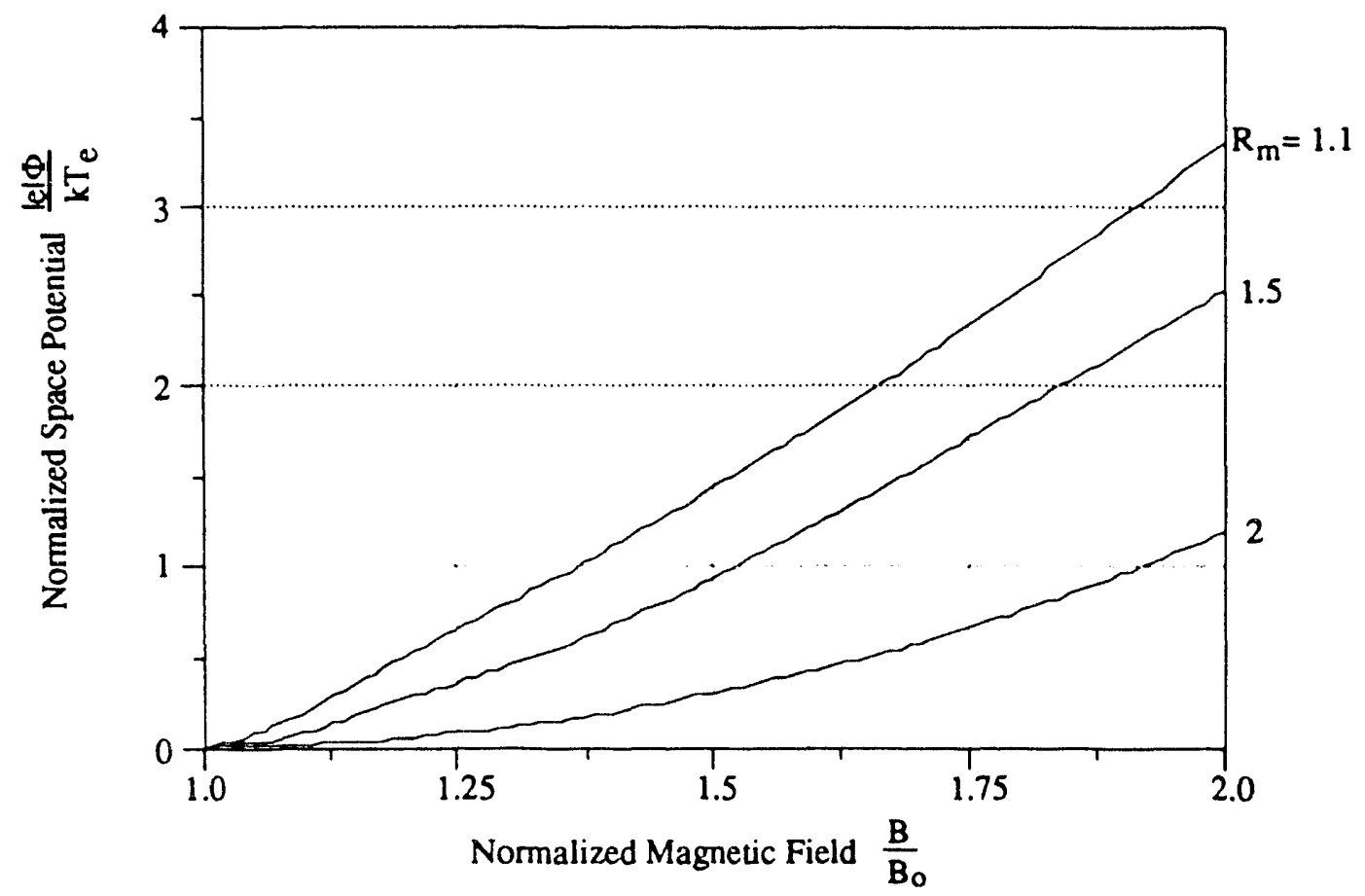

Fig. 2.6 Numerical solution for the more complicated problem of the space potential using the Gaussian quadrature method.

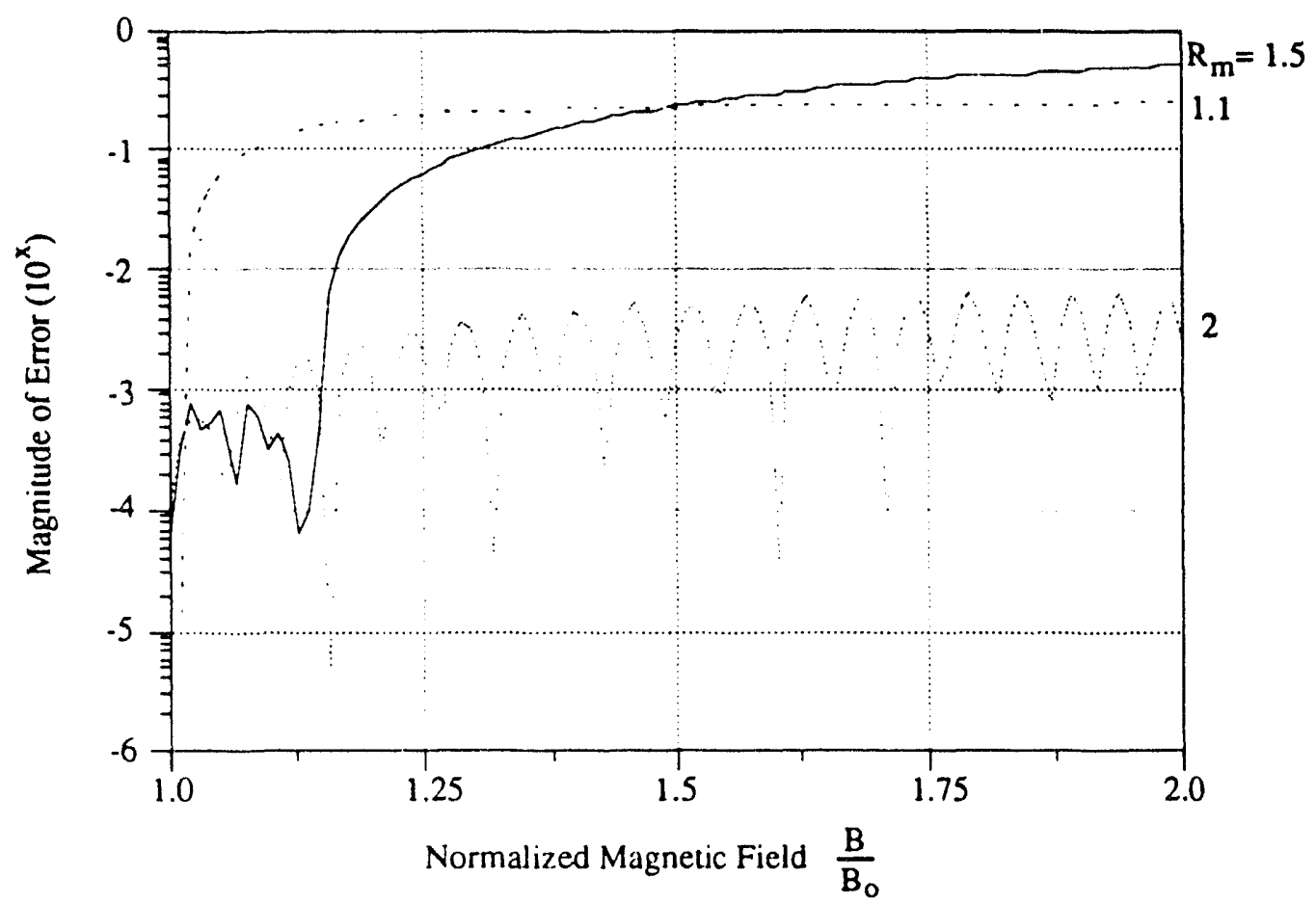

Fig. 2.7 The residual absolute value of eq. 2.35 after the best choice for $\mu_{1}$ ' has been taken. This gives an idea of the accuracy of curves in fig. 2.6 


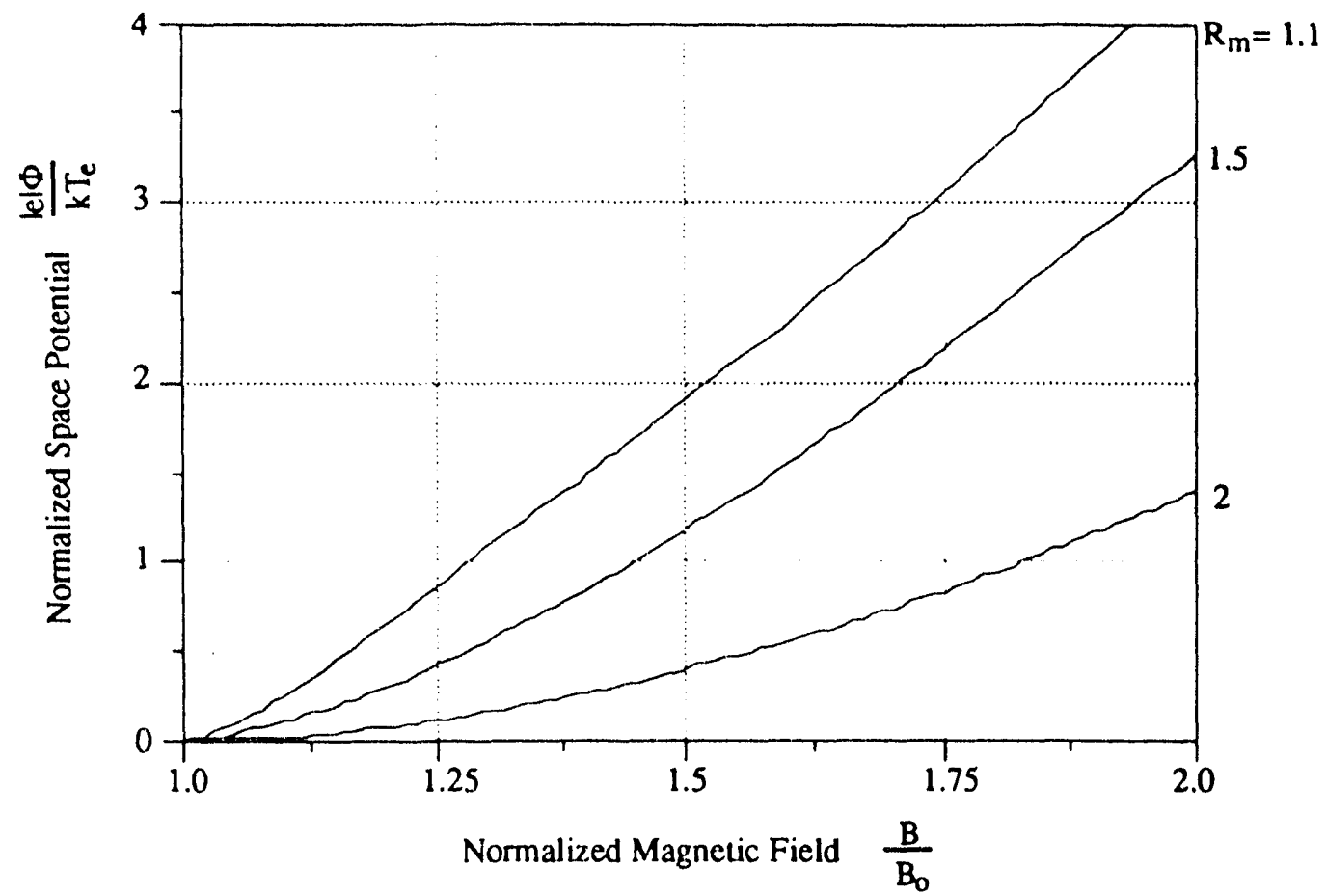

Fig. 2.8 Numerical solution for the more complicated problem of the space potential using Simpson's rule.

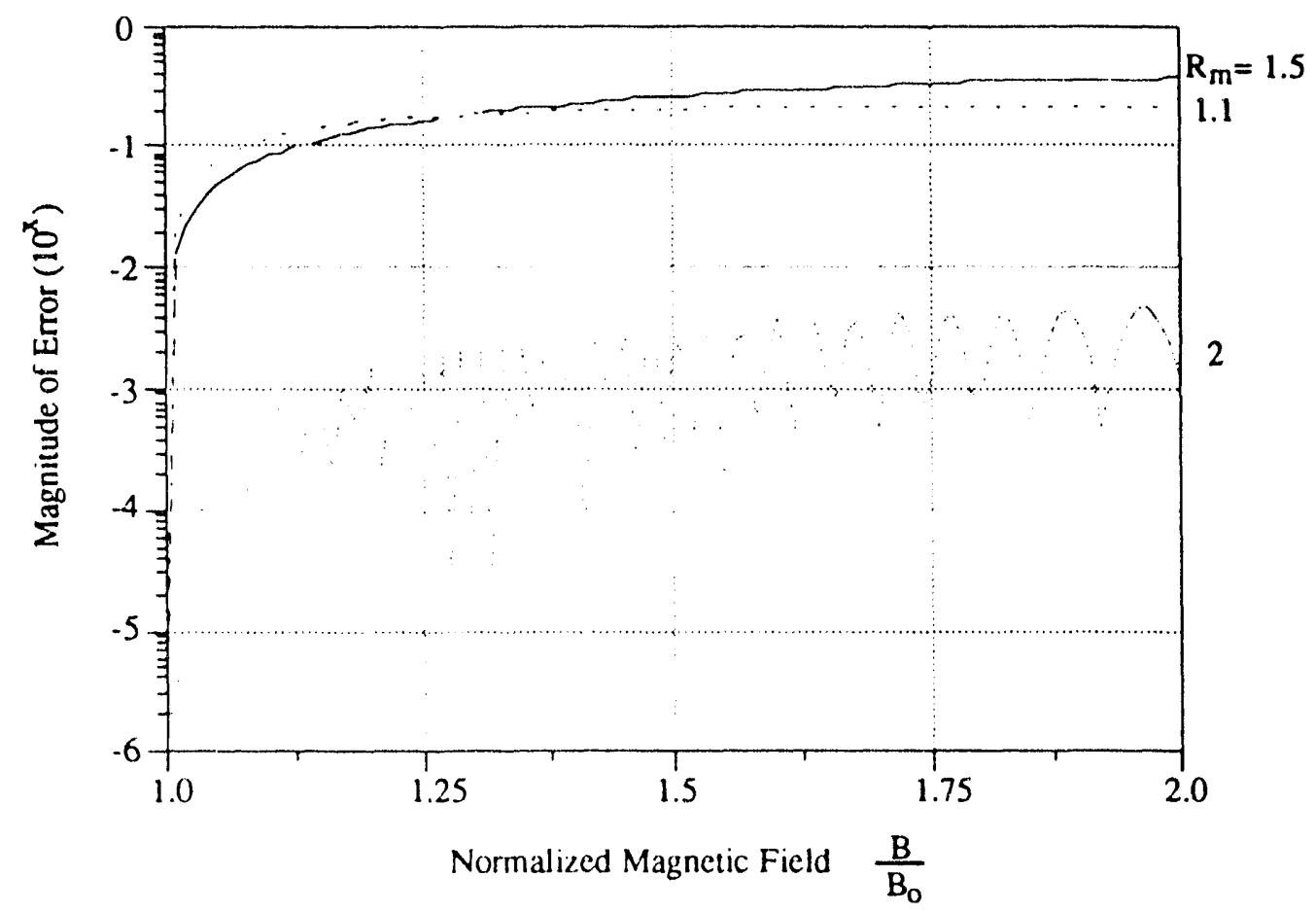

Fig. 2.9 The residual absolute value of eq. 2.35 after the best choice for $\mu_{1}^{\prime}$ has been taken. This gives an idea of the accuracy of curves in fig. 2.8 


\subsection{Relativistic Treatment}

This next section will treat the problem of a transient space potential in a relativistic electron plasma. In this experiment the electron temperatures will only be $50-100 \mathrm{keV}$, hence, not very relativistic. However, it is possible to create plasmas with electron temperatures of a few $\mathrm{MeV}$. In such a plasma it will be important to know if relativistic temperatures places any constraints on the transient potential.

The first step is to pick a relativistic version of the previous model distribution function. The electron velocity, $v$, is replaced with the electron momentum, p. [6]

$$
\begin{gathered}
f_{0}=A \sqrt{p_{\perp}^{2}-K^{2} p_{\|}^{2}} p_{\perp}^{2} \exp \left(-p_{\perp}^{2} / p_{\perp 0}^{2}\right) \\
p_{\perp 0}^{2}=2 m k T_{e}
\end{gathered}
$$

where $f_{0}$ reduces to the nonrelativistic $f_{0}$ except for the factor $A$. This is just a normalization factor that is eventually eliminated from the derivation. The electron density is found by integrating eq. 2.38

$$
n_{e}(B, \phi)=\int_{\delta R} f_{o}\left(p_{\perp}, p_{\|} ; B, \phi\right) p_{\perp} d p_{\perp} d p_{\|}
$$

Here $B$ and $\phi$ are parameters that have not yet been expressed in $f_{0}$. To do so the same procedure is followed: rewrite $f_{0}$ in terms of adiabatic invariants.

$$
\begin{array}{cl}
\sigma=\frac{\mathrm{p}_{\perp}^{2}}{\mathrm{~B}} & \text { magnetic moment } \\
(\mathcal{E}-\phi)^{2}=\mathrm{p}^{2} \mathrm{c}^{2}+\varepsilon_{\mathrm{o}}^{2} & \text { total energy } \\
\mathrm{p}^{2}=\mathrm{p}_{\perp}^{2}+\mathrm{p}_{\|}^{2} \quad \varepsilon_{\mathrm{o}}=\mathrm{m}_{\mathrm{o}} \mathrm{c}^{2} &
\end{array}
$$


Substitute these definitions into $f_{0}$, remembering that $\phi=0$ and $B=B_{o}$ when $t=0$, to get the following

$$
f_{0}=A\left(R_{m} \sigma B_{0} c^{2}+\varepsilon_{o}^{2}+\varepsilon^{2}\right)^{1 / 2} \sigma \exp \left(-\frac{1}{2 k T} \frac{\sigma B_{0} c^{2}}{\varepsilon}\right)
$$

The Jacobian of the transformation is

$$
\mathrm{p}_{\perp} \mathrm{dp}_{\perp} \mathrm{dp}_{\|}=\frac{\mathrm{B}}{2}(\varepsilon-\phi)\left((\varepsilon-\phi)^{2}-\left(\sigma \mathrm{B}_{\mathrm{o}} \mathrm{c}^{2}-\varepsilon_{\mathrm{o}}^{2}\right)\right)^{-1 / 2} \mathrm{~d} \sigma \mathrm{d} \varepsilon
$$

$\sigma \mathrm{B}_{0} \mathrm{c}^{2}$ is a natural grouping of variables and for clarification it will be defined as $\sigma$. Some more common groupings of variables are given abbreviations.

$$
\begin{gathered}
\sigma \equiv \sigma B_{0} c^{2}=\left(p_{\perp} c\right)^{2} \frac{B_{0}}{B} \\
u_{1}=R_{m} \sigma+\varepsilon_{0}^{2} \\
u_{2}=\frac{B}{B_{0}} \sigma+\varepsilon_{o}^{2} \\
u_{3}=\sigma+\varepsilon_{0}^{2}
\end{gathered}
$$

The integration region is similar to before, only now the boundaries are defined by shifted parabolas instead of straight lines. Figs 2.10-12 illustrate this.

The electron density equation, eq. 2.39, can now can be expressed in invariant space, and is transformed to

$$
\mathrm{n}_{\mathrm{e}}(\mathrm{B}, \phi)=\mathrm{AB} \int_{\delta \mathrm{R}} \sqrt{\mathrm{u}_{1}-\varepsilon^{2}} \sigma \exp \left(-\frac{1}{2 \mathrm{k} T} \frac{\sigma}{\varepsilon}\right) \frac{\varepsilon-\phi}{\sqrt{(\varepsilon-\phi)^{2}-\mathrm{u}_{2}}} \mathrm{~d} \varepsilon \mathrm{d} \sigma
$$




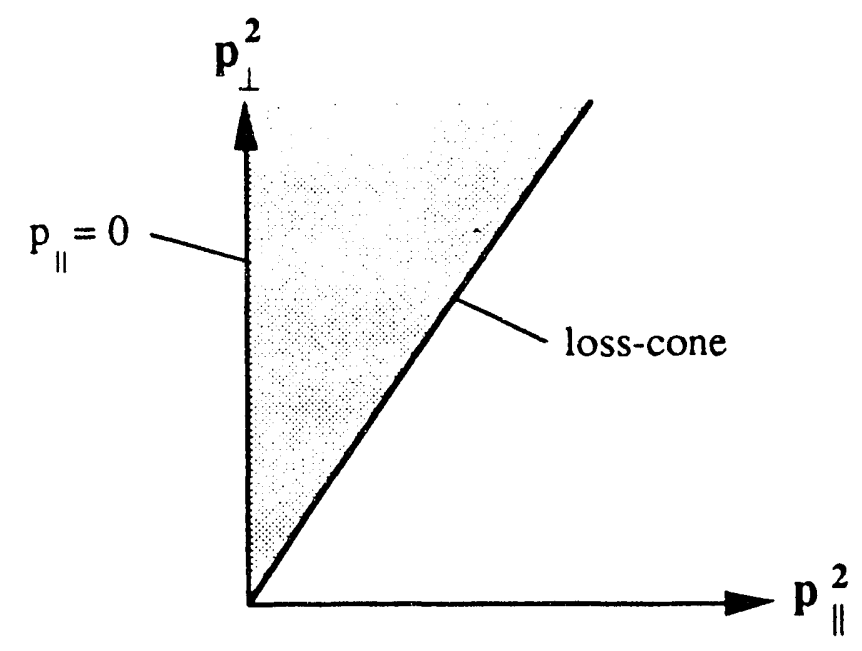

Fig. 2.10 Domain of $\mathrm{f}_{0}\left(\mathrm{p}_{\perp}{ }^{2}, \mathrm{p}_{\|}{ }^{2}\right)$ at $\mathrm{t}=0$.

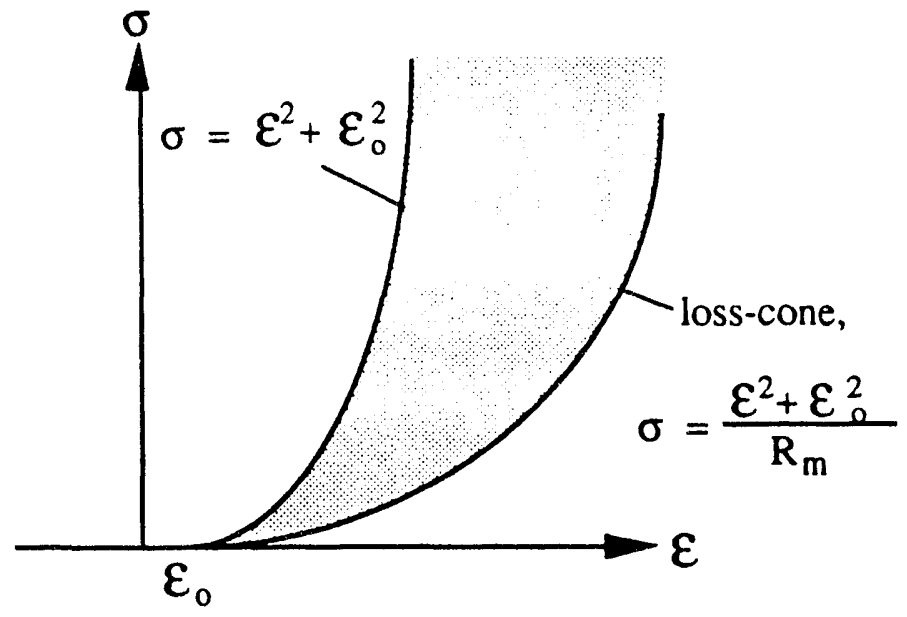

Fig. 2.11 Domain of $\mathrm{f}_{\mathrm{o}}(\sigma, \mathcal{E})$ at $\mathrm{t}=0$.

With the help of figs. 2.11-2.12 the integration region can be seen to be divided into two areas and defined by

$$
\int_{d R}=\int_{0}^{\sigma_{1}} d \sigma \int_{\sqrt{u_{3}}}^{\sqrt{u_{1}}} d \varepsilon+\int_{\sigma_{1}}^{\infty} d \sigma \int_{\sqrt{u_{2}+\phi}}^{\sqrt{u_{1}}} d \varepsilon
$$




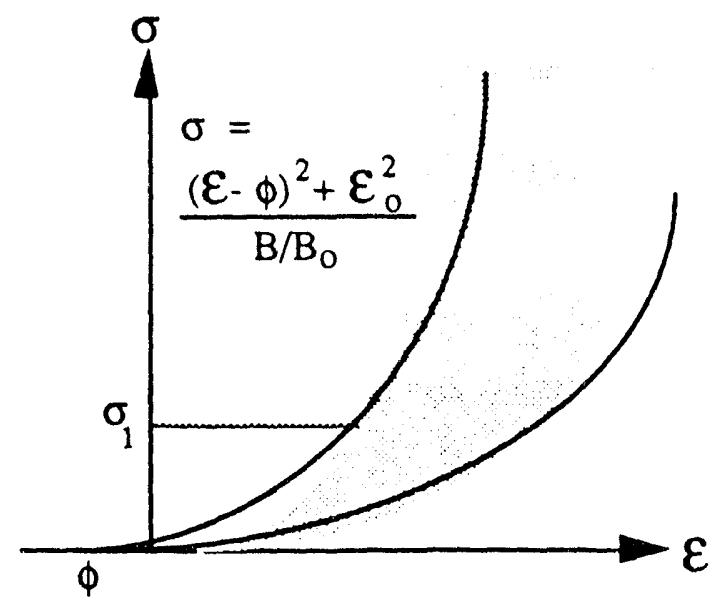

Fig. 2.12 The domain of $f_{0}(\sigma, \varepsilon)$ at $t \neq 0$ is between the solid lines. The shaded region is where $f_{0}$ is still defined.

$\sigma_{1}$ is at the intersection of the left boundaries as shown in fig. 2.12. and is given by

$$
\sigma_{1}=\frac{\left(1+\frac{\mathrm{B}}{\mathrm{B}_{0}}\right) \phi^{2}+|\phi| \sqrt{\frac{\mathrm{B}}{\mathrm{B}_{0}} \phi^{2}+\varepsilon_{0}^{2}\left(1-\frac{\mathrm{B}}{\mathrm{B}_{0}}\right)^{2}}}{\left(1-\mathrm{B} / \mathrm{B}_{0}\right)^{2}}
$$

Unfortunately this integral (eq. 2.44) is too difficult to solve analytically, even when reduced to its initial state, $t=0$, by setting $\phi=0$ and $B / B_{0}=1$. This, of course, is the next step to obtaining a working equation; application of the quasi-neutrality principle. Essentially we take $n(B, \phi)=n\left(B_{0}, 0\right)$. The procedure is to integrate eq. 2.44 numerically with $\phi=0$ and $B=B_{0}$, then use this value to determine the correctness of future values of $n(B, \phi)$ using trial values of $B$ and $\phi$. Now the next thing to think about is numerical methods.

The first thing to notice when considering a numerical integration is the singularity at the lower limit in the second integral. Since numerical methods do not handle singularities well, it is best to make a transformation that amounts to moving back to momentum space. Using the following transformation 


$$
\varepsilon-\phi=\sqrt{\left(p_{\|} c\right)^{2}+u_{2}}
$$

the electron density equation, eq. 2.44 becomes

$$
\begin{gathered}
\mathrm{n}_{\mathrm{e}}(\mathrm{B}, \phi)=A B \int_{\mathrm{R}} \sqrt{\mathrm{u}_{1}-\left(\sqrt{\left(\mathrm{p}_{\|} \mathrm{c}\right)^{2}+\mathrm{u}_{2}}+\phi\right)^{2}} \sigma \exp \left(\frac{1}{2 \mathrm{kT} \sqrt{\left(\mathrm{p}_{\|} \mathrm{c}\right)^{2}+\mathrm{u}_{2}}+\phi}\right) \mathrm{d}\left(\mathrm{p}_{\|} \mathrm{c}\right) \mathrm{d} \sigma \\
\int_{\mathrm{dR}}=\int_{0}^{\sigma_{1}} \mathrm{~d} \sigma \int_{\mathrm{c}_{1}}^{c_{\mathrm{u}}} \mathrm{d} \varepsilon+\int_{\sigma_{1}}^{\infty} \mathrm{d} \sigma \int_{0}^{c_{\mathrm{u}}} \mathrm{d} \varepsilon \\
\mathrm{c}_{\mathrm{u}}=\sqrt{\left(\sqrt{\mathrm{u}_{1}}-\phi\right)^{2}-\mathrm{u}_{2}} \\
\mathrm{c}_{\mathrm{l}}=\sqrt{\left(\sqrt{\mathrm{u}_{3}}-\phi\right)^{2}-\mathrm{u}_{2}}
\end{gathered}
$$

Fig. 2.13 shows what the boundary region might look like at later times during the pulse.

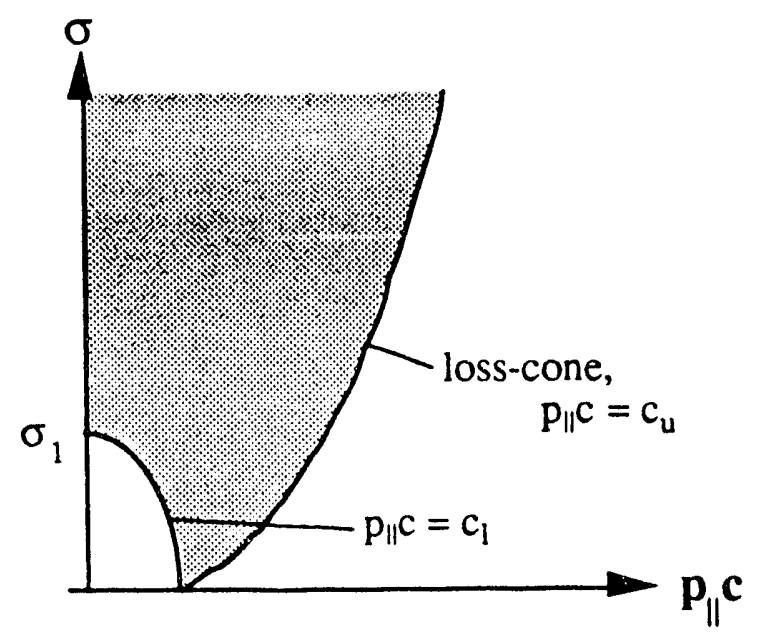

Fig. 2.13 Domain of $f\left(\sigma, p_{\|} c\right)$ at $t \neq 0$.

Since $\sigma$ is proportional to $\left(\mathrm{p}_{\perp} \mathrm{c}\right)^{2}$, it becomes apparent that phase space in fig. 2.13 is just a distorted version of that shown in fig. 2.10 but at later times. The void area in the low energy comer is region $\mathbf{d}$ discussed earlier. The electrons here have left because they are 
accelerated to higher energies by the potential gradients. To the right of the lower boundary curve, $c_{l}$, are the electrons of region $\mathbf{a}$. They are low energy and small pitch angle electrons which are not constrained by the increased magnetic field. They are trapped by the increased local space potential, even though they are beyond the initial loss-cone boundary.

Eq. 2.48 has no singularities in the integrand, but it is still complicated enough that Gaussian Quadrature is not suitable as a numerical method because of the exponential and its complicated argument. This time I only used Simpson's rule. This method has only one basic problem and that is that it only works on finite intervals. To solve this problem I arbitrarily chose a value that was large compared to $\mathrm{kT}^{2}$ to approximate infinity for the $\sigma$ integration. This is because $k T^{2}$ and $\sigma$ are dimensionally equivalent and $k T^{2}$ defines the scale for $\sigma$. For $\mathrm{p}_{\|} \mathrm{c}$ the scale is defined by $\mathrm{kT}$. I chose the sampling interval for each integration proportional to the relevant power of $\mathrm{kT}$ as well.

Because of the factor $\varepsilon_{0}^{2}$, the temperature could not be completely factored out of the problem. Therefore, I picked several values of $k T$ and solved for $\phi(B)$ for each one and normalized it. Ideally for small enough values of $\mathrm{kT}$, the solution should agree with the non-relativistic approach given earlier.

Fig. 2.14 gives the normalized solutions for four different electron temperatures as well as the non-relativistic solution. The $10 \mathrm{keV}$ curve and the non-relativistic curve are a little different, but this might be expected from looking at their fractional errors (error = $\left.\left[n(B, \phi)-n\left(B_{0}, 0\right)\right] / n\left(B_{0}, 0\right)=\Delta n / n_{0}\right)$. The error for the $10 \mathrm{keV}$ solution is $\leq 10 \%$ compared to $40 \%$ for the nonrelativistic solution, but the error is of the same non-oscillating character as shown in fig. 2.9. Exact values of $\phi$ could not be found to solve the electron density equation (eq. 2.48), but the closest real values of $\phi$ were found. These $\Delta n / n_{0}$ errors for the other three relativistic solutions are all less than $0.4 \%$ and oscillatory, indicating that they are exact solutions. Since the curves for $\mathrm{kT}=1 \mathrm{MeV}$ and $10 \mathrm{MeV}$ overlap, it suggests that these solutions represent the extreme relativistic limit. 
The most interesting feature of the relativistic behavior is the negative space potential for low applied fields. This might have something to do with the choice of electron distribution model. In any event these variations in the normalized space potential do not effectively reduce the gains to be made in the net space potential by simply raising the electron temperature, so long as the applied field is high enough to produce a positive potential.

Figs. 2.15-16 give the same relativistic solutions for two other mirror ratios of 1.1 and 2. None of the solutions given for $R_{m}=1.1$ are exact. The $\Delta n / n_{0}$ errors are about $50 \%$. The solutions for $R_{m}=2$ are exact with fractional errors less than $0.4 \%$. The negative space potential behavior becomes very extreme in this case. 


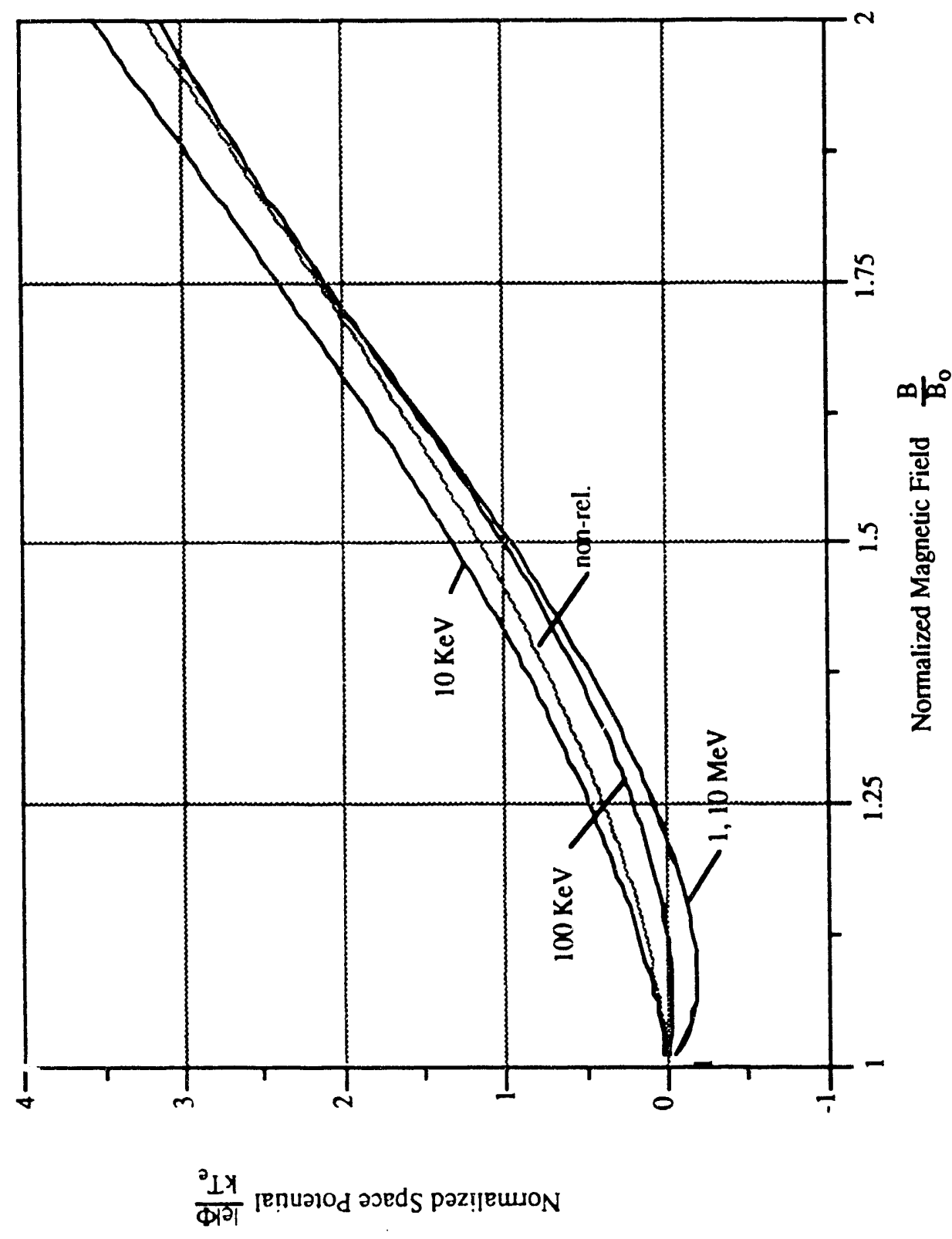

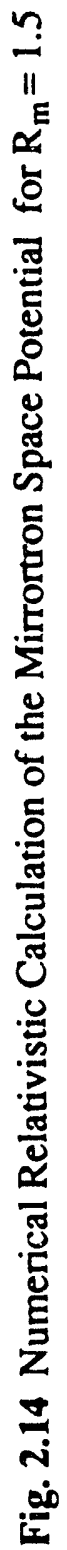




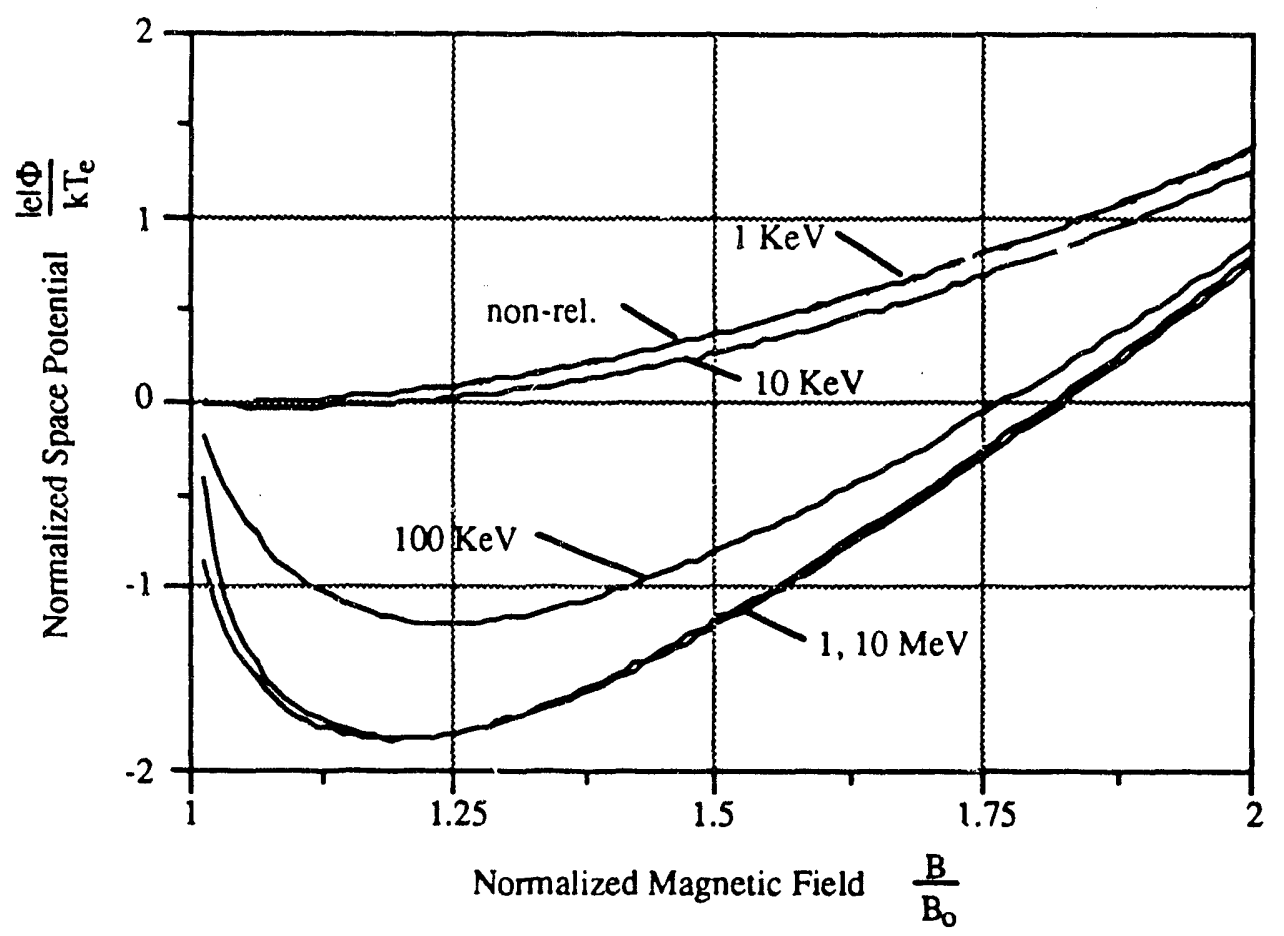

Fig. 2.15 Numerical solution of the relativistic space potential for $R_{m}=2$

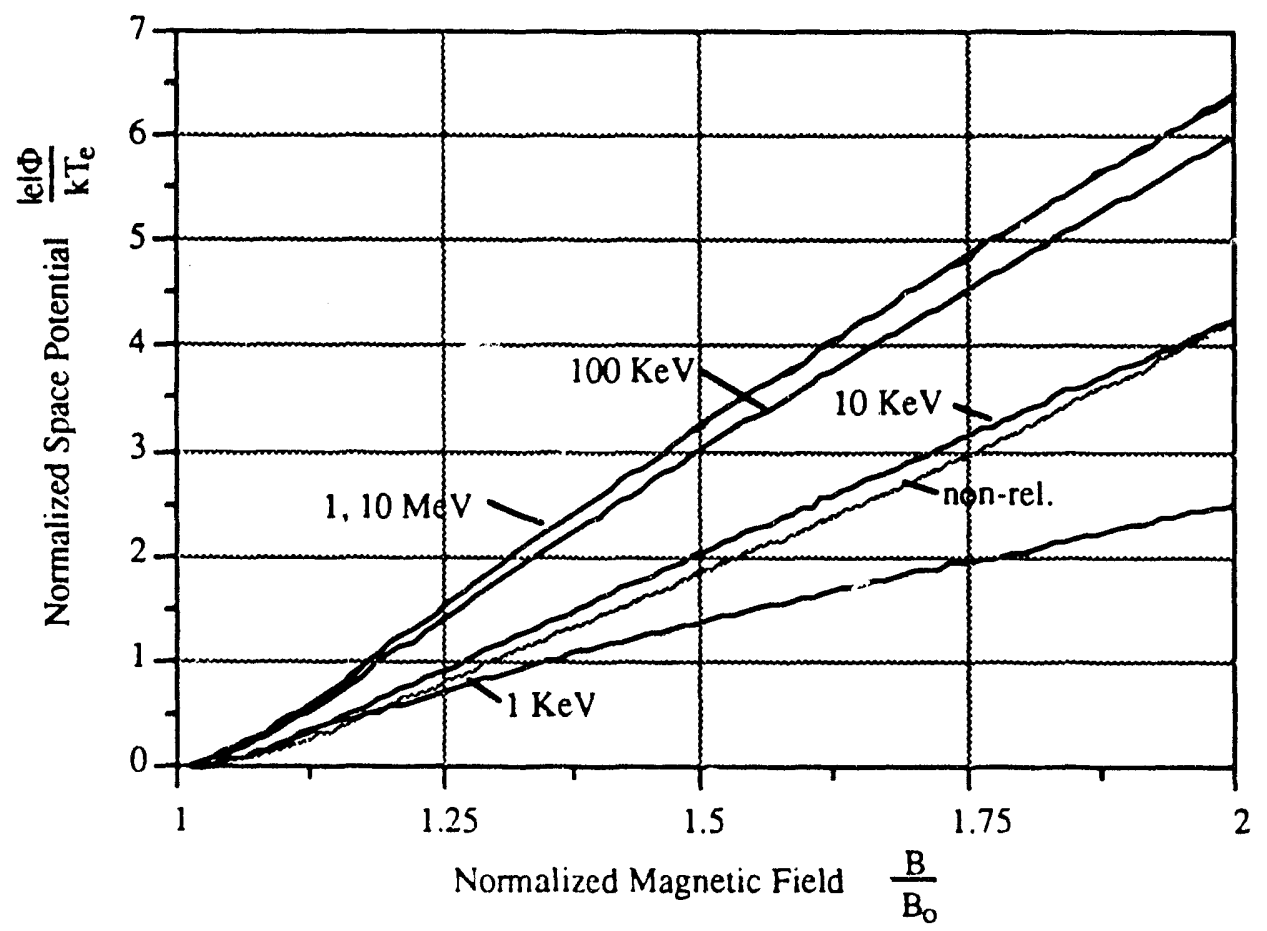

Fig. 2.16 Numerical solution of the relativistic space potential for $R_{m}=1.1$ 


\section{Chapter III Design of the Experiment}

The initial design of the Mirrortron experiment is discussed in this chapter. The purpose of the Mirrortron experiment is to create, heat, and trap hot electrons in a mirror confinement system. This contains a description of the vacuum and gas feed systems, the magnetic fields, and the electron cyclotron resonance heating (ECRH) system. The eventual goal is to create a loss-cone distribution which will be achieved during the decay of the plasma from the steady-state. For this reason a timing control box is used to control the shutoff of the ECRH and gas flow, and it is described at the end of the chapter.

\subsection{Vacuum System}

\subsubsection{Shape and Dimensions}

To create a mirror confinement system the basics were collected: a vacuum vessel consisting of three cylinders $50.8 \mathrm{~cm}$ (20 in.) long and $26.7 \mathrm{~cm}$ (10.5 in.) in diameter. The plasma chamber is a $152.4 \mathrm{~cm}(60 \mathrm{in}$.) long cylinder. The chamber is oriented in an eastwest direction. On the west side a one inch thick solid flange is mounted containing 91 
holes for vacuum pumping. Each hole is $1.9 \mathrm{~cm}(0.75 \mathrm{in}$.$) in diameter. The purpose of this$ flange is to provide a surface that defines the end of the microwave resonant cavity. An additional $50.8 \mathrm{~cm}$ (20 in.) long cylinder connects the chamber to a vacuum pump. Fig. 3.1 is a diagram of the vacuum vessel in relation to the electromagnets.

An adapter flange is mounted on the east end of the vessel that connects to a smaller solid flange with $19,1.3 \mathrm{~cm}(0.5 \mathrm{in}$.) diameter holes drilled in it. Tubes that are $22.9 \mathrm{~cm}(9$ in.) long are press fit into the holes, and these tubes are used for collimation of the gas into a molecular stream. These tubes are placed inside a $30.5 \mathrm{~cm}$ (12 in.) long plenum chamber that is $10.1 \mathrm{~cm}$ (4 in.) in diameter. A blank flange capped off the end in the first phase, but in the second phase the electron gun was mounted at this position. A $0.6 \mathrm{~cm}(0.25 \mathrm{in}$.) diameter tube feeds into the side of the plenum chamber and carries the supply gas. Located on the east end adapter flange are four vacuum sealed ceramic inserts. It is through these inserts that microwave radiation will couple into the cavity. Fig. 3.2 is a close-up picture of the east end of the vacuum vessel.

\subsubsection{Gas Flow}

\subsubsection{Gas Supply}

Gas flows into the plenum chamber through tubing from a gas feed can. Argon and hydrogen were the optional gasses used. The can has a 3.8 liter volume. Gas is fed from a source bottle into this can in controlled amounts so that the working pressure in the can is in the millitorr or low torr range. In line between the gas feed can and the plenum chamber is a needle type, hand valve. The purpose of the can is to maintain a reservoir of gas with a low pressure differential across the needle valve so that the gas flow rate may be more easily controlled with the valve. Downstream from the hand valve is an electrically controlled, solenoid valve. This valve is used with the timing control box to stop the gas flow and initiate the plasma decay process. 


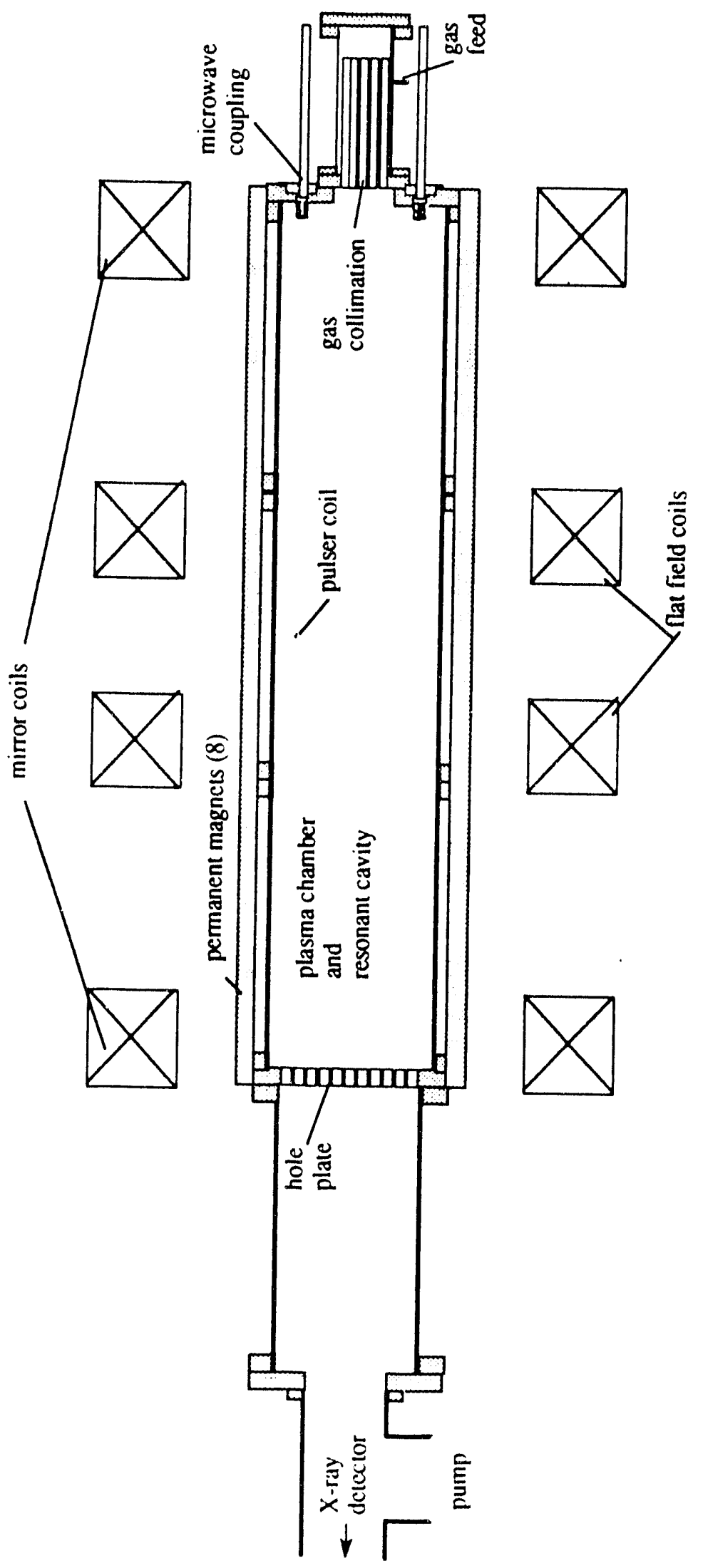

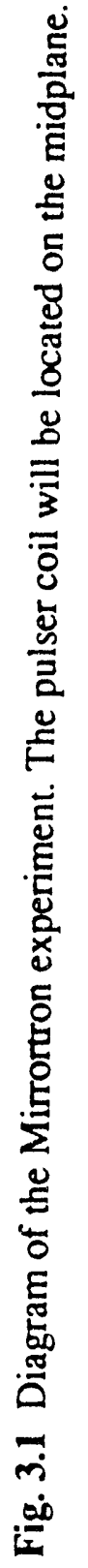




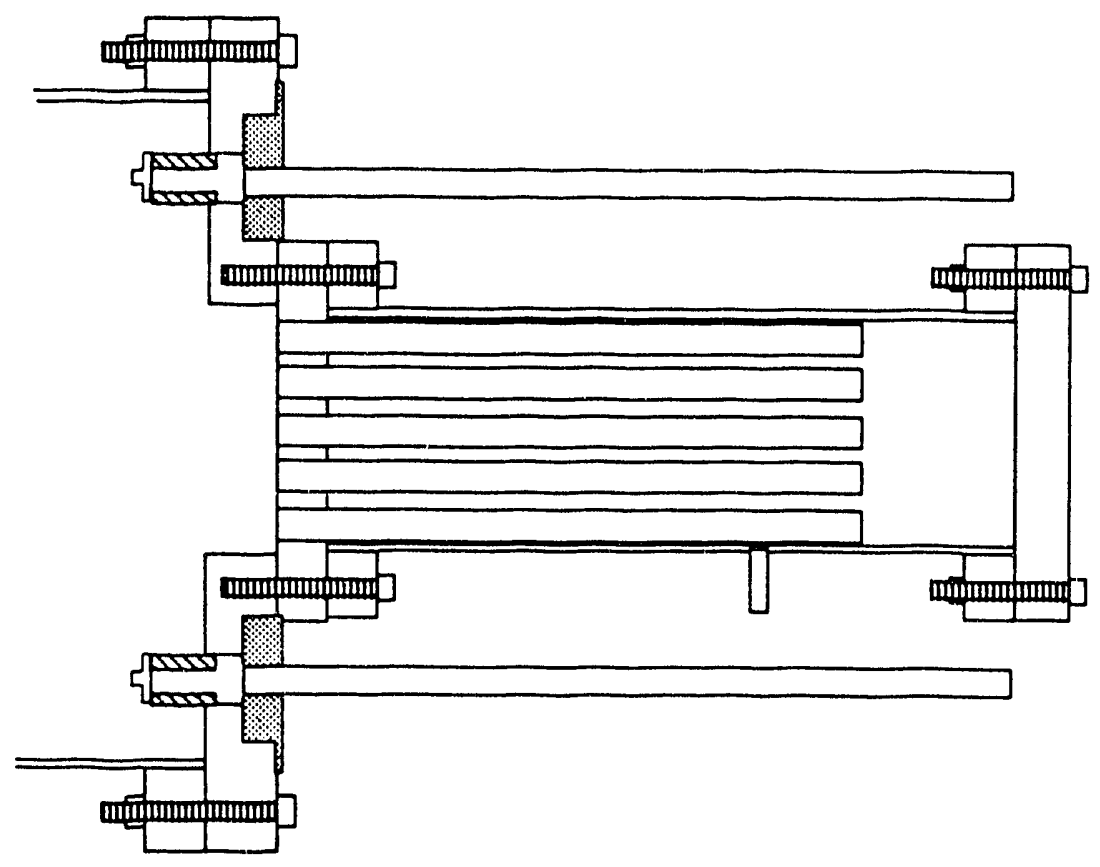

Fig. 3.2 East end of the experiment showing the plenum chamber with collimation tubes. Also shown are the ceramic feedthroughs allowing microwave radiation to pass.

\subsubsection{Scattering Off Neutrals}

During the plasma decay phase there are two concerns about high background gas pressure. Since the neutral gas density is expected to be at least ten times higher than the plasma density, there exists the possibility of increased decay of the hot component from scattering off the neutrals. The other concern is of a continuing cold electron population. Even after the cold electrons leave the plasma, there will be ionization of background gas by the hot electrons.

Decay from the scattering off neutrals is not negligible because of their high density. The neutral density is expected to be $10^{11} \mathrm{~cm}^{-3}$, based on the hydrogen gas pressure in the vacuum chamber, perhaps about 10 times greater than the cold electron density and about 100 times the hot electron density. To examine the scattering, the characteristic times for an 
electron to scatter $90^{\circ}$ are listed in Tables 3.1, which has coulomb scattering times, and Table 3.2, which has neutral scattering times.

Equation 3.1 is derived from $\tau=\frac{1}{n_{c} \sigma v}$ where $\sigma$ is the coulomb cross-section, and $v$ is the electron velocity. [8]

$$
\tau_{e}=1.1410^{10} \frac{T^{3 / 2}}{n_{e} \ln \Lambda}
$$

This equation is used to calculate the values in Table 3.1 for a range of electron temperatures and densities.

\section{Table 3.1}

Characteristic Time for $90^{\circ}$ Coulomb Scattering of Electrons

\begin{tabular}{||c|c|c|c|c|}
\multicolumn{1}{c}{$T_{\mathrm{e}}$} & \multicolumn{4}{c|}{$\mathrm{n}_{\mathrm{c}}\left(\mathrm{cm}^{-3}\right)$} \\
\cline { 2 - 5 } \multicolumn{1}{c|}{$(\mathrm{keV})$} & $10^{8}$ & $10^{9}$ & $10^{10}$ & $10^{11}$ \\
\hline 0.01 & $3.2 \mathrm{~ms}$ & $0.36 \mathrm{~ms}$ & $38 \mu \mathrm{s}$ & $6 \mu \mathrm{s}$ \\
\hline 0.1 & $85 \mathrm{~ms}$ & $9.0 \mathrm{~ms}$ & $0.95 \mathrm{~ms}$ & $0.10 \mathrm{~ms}$ \\
\hline 1 & $2.3 \mathrm{~s}$ & $0.25 \mathrm{~s}$ & $26 \mathrm{~ms}$ & $2.7 \mathrm{~ms}$ \\
\hline 50 & $800 \mathrm{~s}$ & $90 \mathrm{~s}$ & $9.0 \mathrm{~s}$ & $1.9 \mathrm{~s}$ \\
\hline
\end{tabular}

In the case of an electron scattering off a neutral atom, a slightly different form of eq. 3.1 could be used. For large angle scattering, the impact parameter is much smaller than the atomic size and the shell electrons are outside the interaction region. Hence large angle scattering can be described by coulomb forces. The difference is that $\ln \Lambda$ in eq. 3.1 is ignored because it accounts for multiple long distance interactions that do not occur in neutral scattering. The distance of closest approach for $90^{\circ}$ scattering will be smaller than 
the Bohr radius for energies greater than $10 \mathrm{eV}$. Hence all the values in Table 3.2 are calculated using eq. 3.1 without $\ln \Lambda$ and using the neutral density for $n_{e}$. [9]

Table 3.2

Characteristic Time for Scattering Off Neutrals

\begin{tabular}{||c|c|c|c|}
\multicolumn{1}{c}{$T_{\mathrm{e}}$} & \multicolumn{3}{c|}{$\mathrm{n}_{\mathrm{n}}\left(\mathrm{cm}^{-3}\right)$} \\
\cline { 2 - 4 }$(\mathrm{keV})$ & $10^{9}$ & $10^{10}$ & $10^{11}$ \\
\hline 0.1 & $360 \mathrm{~ms}$ & $36 \mathrm{~ms}$ & $3.6 \mathrm{~ms}$ \\
\hline 1 & $11 \mathrm{~s}$ & $1.1 \mathrm{~s}$ & $0.11 \mathrm{~s}$ \\
\hline 50 & $4000 \mathrm{~s}$ & $400 \mathrm{~s}$ & $40 \mathrm{~s}$ \\
\hline
\end{tabular}

These values show that the hot electron component should remain trapped for many seconds and that the neutral gas should not result in a faster decay of the plasma than the natural coulomb collisional process. This also demonstrates that a few hundred ms after the shutoff of the gas and ECRH would be a good time to apply the pulsed magnetic field because most of the cold electrons up to $1 \mathrm{keV}$ will have escaped, leaving most of the hot component behind.

\subsubsection{Background Ionization}

In the decay phase the cold electrons escape quickly, but the ionization of background gas by the lingering warm electrons will still continue. The ionization cross section, $\langle\sigma v\rangle_{i}$, peaks at $2.010^{-8} \mathrm{~cm}^{3} / \mathrm{s}$ for 100 to $400 \mathrm{eV}$ electrons but decreases slowly for higher energy electrons. The ionization rate also depends, of course, on the density of the background gas. The characteristic ionization time $\tau_{\text {ionize }}$ is $\frac{1}{\left.n_{n}<\sigma v\right\rangle_{i}}$. The equation that estimates the evolution of the cold electron density is 


$$
\frac{d n_{e(\text { cold })}}{d \tau}=-\frac{n_{e(\text { cold })}}{\tau_{e(\text { cold })}}+\frac{n_{e(\text { hol })}}{\tau_{\text {ionize }}}
$$

In general $n_{e(h o t)}$ will change slowly during a characteristic decay time $\tau_{e(\text { cold })}$ such that the second term in eq. 3.2 can remain constant. Then the steady solution after the decay of the initial cold component is $n_{\mathrm{e}(\text { cold })}=\left(\tau_{\mathrm{e}(\text { cold })} / \tau_{\text {ionize }}\right) \mathrm{n}_{\mathrm{e}(\mathrm{hot})}$. Table 3.3 gives the cold electron density for two temperature components of the plasma while the background gas density is still high. $\tau_{\text {ionize }}$ is calculated for a density of $10^{11}$ hydrogen atoms. An estimate of $n_{e(h o t)}$ is given in the table, and $\tau_{\mathrm{e} \text { (cold) }}$ is determined using values from Table 3.1.

From Table 3.3 it is apparent that $1 \mathrm{keV}$ electrons can produce a cold electron density equal to the density of hot electrons $\left(10^{9} \mathrm{~cm}^{-3}\right)$. However, even these cold electrons last only as long as the $1 \mathrm{keV}$ electrons do, which is about $25 \mathrm{~ms}$. The hot electrons can produce cold electrons that are a significant fraction of its own density for as long as they last and as long as the high background gas pressure lasts. Background ionization has not clearly been eliminated as a potential problem, hence the necessity for fast pumping is shown.

Table 3.3

\begin{tabular}{c|c|c|c} 
& \multicolumn{4}{|c}{ Ionization Parameters } \\
$\mathrm{Te}(\mathrm{keV})$ & $\tau_{\text {ionize }}(\mathrm{ms})$ & $\mathrm{n}_{\mathrm{e}(\text { hol })}\left(\mathrm{cm}^{-3}\right)$ & $\mathrm{n}_{\mathrm{e}(\mathrm{cold})}\left(\mathrm{cm}^{-3}\right)$ \\
\hline 1 & 0.59 & $10^{10}$ & $510^{9}$ \\
50 & 3.3 & $10^{9}$ & $510^{8}$
\end{tabular}

\subsubsection{Pumping}

\subsubsection{Pump Down Time}

To calculate the pump down time of the plasma chamber it will be necessary to know the pumping speed and relevant conductances. The vacuum pump on the Mirrortron is a 
very fast pump rated at 2000 liter per sec for air. For $\mathrm{H}_{2}$ it is only $1500 \mathrm{~V} / \mathrm{s}$. Typical operating pressures are about $1.010^{-6}$ torr as measured by an ion gauge just over the pump. The gauge is calibrated for air, so an approximate correction factor of 2 for hydrogen gives $2.010^{-6}$ torr. The product of the pumping speed and pressure gives the throughput. Knowing the throughput and the conductance to any region in the vessel, the pressure can be calculated for that region. The conductance to the chamber, including through the 91 hole plate, is calculated to be approximately $1000 \mathrm{l} / \mathrm{s}$. Hence, the average typical background pressure in the plasma is $3.210^{-6}$ torr. The base pressure at the gauge is $210^{-7}$ torr of air or $610^{-7}$ torr in the chamber.

The effective pumping speed on the chamber is $S=600 \mathrm{~V} / \mathrm{s}$. If there were no gas source, the chamber pressure would drop exponentially with a characteristic time of $\tau_{p}=$ $S / N$ where $V=85.1$ liter is the chamber volume, so that $\tau_{p}=140 \mathrm{~ms}$.

There is a concern about gas continually flowing into the plasma chamber from the higher pressure plenum chamber and the gas feed tube after the solenoid switch has been closed. However, it turns out that these volumes are small enough that if all the confined gas were instantly transferred to the main chamber that it would only increase the pressure by $510^{-7}$ torr. Since this gas is pumped out through the small conductance of the collimating tubes, the peak pressure increase is even less than this. The characteristic pump down time of the plenum chamber is about $20 \mathrm{~ms}$.

\subsubsection{Collimated Gas Flow}

A pump down time of $140 \mathrm{~ms}$ maybe adequate for our purposes. However, a simple method to improve this time further can be employed without extra effort. This was thought of as a general way of improving the Mirrortron operation. It involves collimating the gas flow so that the molecules stay concentrated near the axis.

A molecular stream can be created by a tube with a length $L$ and a radius a if the ratio $\mathrm{L} / \mathrm{a}$ " 1 and if the pressure is low enough that molecular flow is allowed. In other words, 
the mean free path of the molecules, $\lambda_{\mathrm{mfp}}$ must be much greater than the tube dimensions. The pressure in the plenum chamber is calculated to be $310^{-5}$ torr, so that $\lambda_{\operatorname{mfp}}$ for $\mathrm{H}_{2}$ is $650 \mathrm{~m}$. The half angle for divergence of the molecular beam is given by [10]

$$
\frac{\theta}{2}=1.68 \frac{\mathrm{a}}{\mathrm{L}}
$$

The angle of divergence gives the full width of the half maximum of molecular flow (molecules through a unit area per second).

The collimation tubes are $22.9 \mathrm{~cm}$ ( $9 \mathrm{in}$.) long and have a radius of $0.56 \mathrm{~cm}(0.22 \mathrm{in})$. For this geometry, $\theta=7^{\circ}$. This describes a cone that casts a circle of radius $6.22 \mathrm{~cm}(2.45$ in.) on the 91 hole pump out plate. The collimation tubes are in an array of 19 . This means that the total half angle pattern on the hole plate is a circle of radius $10.0 \mathrm{~cm}$ (3.95 in.). Fig. 3.3 shows the geometry.

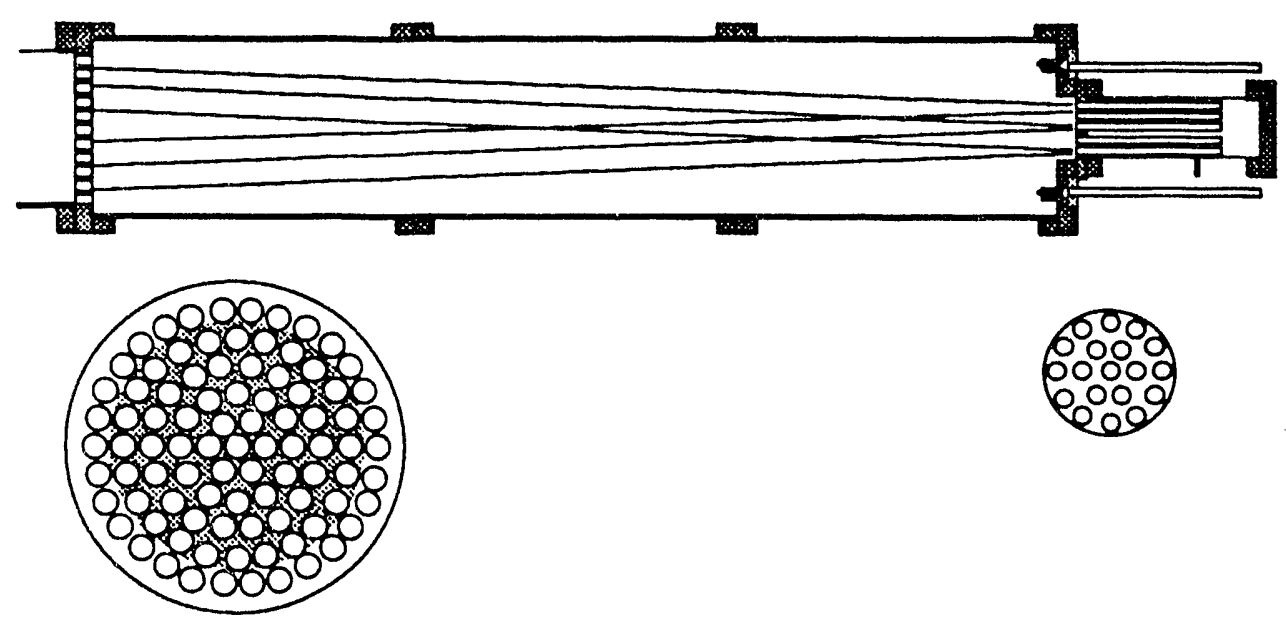

Fig. 3.3 Geometry of the gas collimation. On the right is the hole pattern for the collimation tubes. On the left is the hole pattern in the ventilation flange for the gas pump-out. The shaded area indicates where the halfmaximum gas flux lands. 
Of course, not all the molecules will pass through the holes on the first shot; $45 \%$ will be reflected back into the main chamber and contribute to the vessel background pressure. However, it can be seen that $55 \%$ of the gas in the beam will leave the chamber in a single transit time of $1 \mathrm{~ms}$ for $\mathrm{H}_{2}$ gas at room temperature. By use of collimation the pressure would quickly drop by one half, and the remaining pressure would then decay in the standard pump out time.

\subsection{Mirror Field}

At this point the shape and magnitude of the magnetic field has yet to be decided upon. The basic requirements are that the mirror field fit inside the vacuum vessel, and it be of a configuration that assures MHD stability. A mirror ratio of about 1.5 seems appropriate, as suggested by theory.

Another desirable feature could also be designed into the field, i.e., a uniform magnetic field at the midplane. This would be helpful because it more closely matches the conditions assumed in the theory. The transient potential derivation assumed a uniform background field where a localized pulsed field was applied. The axial magnetic field should be designed as a long uniform field with short confining mirror fields at the ends.

\subsubsection{Solenoid Electromagnets}

The main magnetic field is produced by four large electromagnets. Each electromagnet has a cross section of 6 by 6.5 inches with a 15 inch mean radius. Each one consists of 144 turns of water cooled copper conductor. The coils are rated to produce 2.3 gauss of field at their centers for each ampere of current in the coil.

One pair of electromagnets is used to produce the mirror fields, and the coils are placed so that the field peaks are a little inside the end wall of the main vacuum chamber. The chamber is $152 \mathrm{~cm}$ (60 in.) long and the centers of the coils are $140 \mathrm{~cm}(55 \mathrm{in}$.) apart. The second pair of coils is to be used like Helmholtz coils to create a flat field. The separation 
between the Helmholtz coils is equal to the coil radius, i.e., $38 \mathrm{~cm}$ (15 in.). Using the EFFI computer code to analyze the magnetic field produced by the electromagnets, it was decided that a separation of $36 \mathrm{~cm}$ (14 in.) was best.

Two power supplies were obtained. One was rated for $1 \mathrm{kA}, 100 \mathrm{~V}$. Another was rated for $500 \mathrm{~A}, 16 \mathrm{~V}$. It was planned to use these power supplies to run $150 \mathrm{~A}$ in the Helmholtz coils and $450 \mathrm{~A}$ in the mirror field coils. These values were used in an EFFI calculation and the result is plotted in fig. 3.4. It shows the $\mathbf{B}$ field on axis with a midplane field of $700 \mathrm{~g}$ and a mirror field of $1085 \mathrm{~g}$. This mirror field has a mirror ratio of 1.55 , which is very close to what was intended. A flat field effect is achieved out to $30 \mathrm{~cm}$ from the midplane where the maximum variation is under $4 \%$.

The resonance zone for $2.4 \mathrm{GHz}$ microwaves occurs at a field strength of $860 \mathrm{~g}$. This point is about $47 \mathrm{~cm}$ from the midplane. It is expected that the plasma will uniformly fill the region between the two resonance zones, and spatially localized plasma distributions will be avoided. Such plasmas might be formed if the resonance zone was too near the midplane, and these distributions might lead to instabilities, for example, Whistler modes.

In the experiment the maximum current provided by the supplies was only 440 and 120 amps. This might be partly explained by the added resistance of the welding cable connecting the supplies to the coils. Later on, different power supplies were acquired that could provide the full current required.

\subsubsection{Energy Confinement Calculation}

One particular concern in the design of the mirror field is the issue of energy confinement. Since the electrons of the plasma will be very energetic, relative bounce frequencies will be very short. There exists a possibility that the magnetic field in the electron reference frame will change fast enough compared to cyclotron periods, as the electron bounces from reflection point to reflection point, that non-adiabatic effects could occur. This is quantified by the parameter $\alpha=v / \omega_{c} L$, where $v$ is the electron velocity and $L$ 


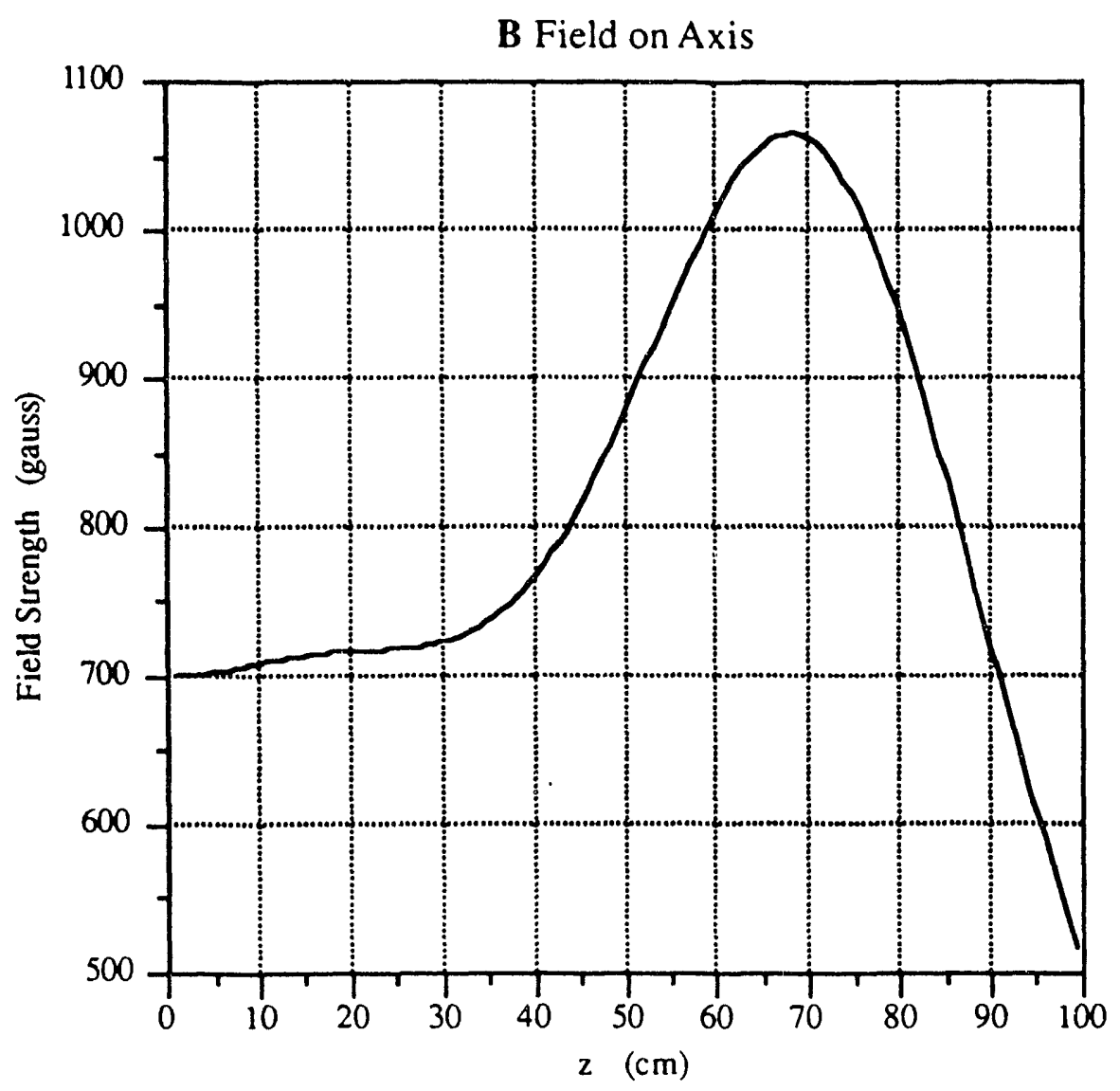

Fig. 3.4 A calculation of the Mirrortron mirror field. The midplane field is $700 \mathrm{~g}$. The peak field is $1085 \mathrm{~g}$ at $\mathrm{z}=69 \mathrm{~cm}$. The mirror ratio is 1.55 .

is the scale length over which the magnetic field varies. If it is not true that $\alpha \ll 1$ then these effects can lead to enhanced particle losses. If these lusses dominate over collisional losses then the optimum plasma energy confinement is compromised.

A calculation was done to find what the maximum energy the electrons could have in this mirror field before they would begin to escape by non-collisional processes. The first step in this calculation will be a comparison of relaxation times for the various loss processes. The characteristic time for coulomb scattering of plasma particles, $\tau_{\mathrm{e}}$, is given in eq. 3.1. In this case the temperature represents the energy of the electron.

The characteristic time for non-adiabatic processes is[11] 


$$
\tau_{\mu}=\frac{\pi \lambda \mathrm{L}}{\mathrm{A}^{2} \mathrm{v}} \mathrm{e}^{(2 \mathrm{~K} / \alpha)}
$$

where $\lambda$ is the ratio of the electron's perpendicular velocity at the midplane to its total velocity. The requirement for electrons trapped in a mirror with $R_{m}=1.5$ is $\lambda \geq 0.8$. The quantities $A$ and $K$ depend on the spatial variation of the magnetic field. $K$ is actually a function of $\lambda$. Assuming an azimuthally symmetric field with a parabolic axial variation, $B(z)=B_{0}\left(1+z^{2} / L^{2}\right)$, then $A=4$ and $K=1$ if $\lambda=0.8$

Collisional losses dominate when $\tau_{e}<\tau_{\mu}$. An upper energy limit corresponds to $\tau_{e}=$ $\tau_{j \mu}$. This leads to a transcendental equation for the electron energy. To solve this, some parameters are chosen. The greatest restriction is on a barely trapped electron, hence, $\lambda=$ 0.8. For the parabolic approximation to the magnetic field, the parameters are $B_{0}=700 \mathrm{~g}$ ? i $\mathrm{L}=93 \mathrm{~cm}$. An electron density of $10^{10} \mathrm{~cm}^{-3}$ is used, and $\ln \Lambda$ is 30 for these low densities and high temperatures.

The result: is that the upper energy limit is $950 \mathrm{keV}$, and the characteristic loss time for both processes at this energy is $10^{3} \mathrm{sec}$. For lower electron densities the energy limit is lower, how cver, the typical loss times become even longer. So even for energetic electrons in the tail of the disticution, additional non-coilisional losses are not expected to be a problem in terms of maintaining a hot electron distribution.

\subsection{MHD Stability}

Having come up with a means c: producing an axisymmetric mirror field, attention must now be paid to the magnetohydrodynamic stability of the plasma confinement. The major concem here is stopping the flute-instability. Other instabilities can occur for high $\beta$ plasmas. This is not a concern in this experiment. The maximum $\beta$ her: is expected to be about $4 \%$. 
The flute-instability is characterized by charge separation in an azimuthal direction on the surface of the plasma. This is due in part to the flux lines curving around the center of the plasma. The field lines near the mirrors curve in toward the axis, and as plasma travels along these lines, the accumulated charge is dispersed. In general, the overall curvature seen by the average plasma particle is negative, thus, the instability is allowed to grow. Two major steps can be employed to prevent charge accumulation and the flute-instability. One is flux line tying, and the other is a minimum-B field configuration. Another stabilizing effect may result from the nature of the hot electron plasma. This effect is the result of a non-negligible gyruradius.

\subsubsection{Finite Orbit Stabilization}

In the review article by Post [12], the condition for finite orbit stabilization is given as follows

$$
\frac{r_{g}}{r_{p}}>\frac{\sqrt{m}}{m-1} \frac{r_{p}}{L}
$$

where $r_{g}$ is the electron gyroradius and $r_{p}$ is the plasma radius and $L$ is the plasma length. The mode number $m$ describes the number of lobes in the flute. Inserting typical plasma parameters $\left(50 \mathrm{keV}, 700 \mathrm{~g}, \mathrm{r}_{\mathrm{g}} \approx 1 \mathrm{~cm}, \mathrm{r}_{\mathrm{p}} \approx 10 \mathrm{~cm}, \mathrm{~L} \approx 100 \mathrm{~cm}\right)$ in eq. 3.5 suggest that the condition for stabilization can be satisfied for modes $m>3$. The lowest two modes correspond to bulk movement of the plasma. Line tying should be effective in preventing this kind of behavior.

\subsubsection{Line Tying}

An often used method for stabilization of the flute-instability is line tying. This is when magnetic flux lines intercept conducting plates at or near the mirror throats. Conductivity along the field lines is important, so that charge collecting on the plasma surface can be 
essentially shorted out. Although the purpose of magnetic mirrors is to isolate the plasma from the wall, effective electrical contact can be achieved if its beta factor is not too high.

In the Mirrortron experiment the end flanges of the vacuum chamber and microwave cavity serves as conducting plates intersecting the flux lines. The mirror peaks are located $6.4 \mathrm{~cm}$ (2.5 in.) inside these end walls. In the event that the hot electron plasma is still electrically isolated, the addition of a multipole field will cause the field lines to diverge outward and meet the cylindrical walls of the vacuum vessel before the mirror point of the axisymmetric mirrors. Hence the electrical conduction will improve.

\subsubsection{Minimum-B Field}

\subsubsection{Octupole Field}

In general, a transverse multipole field is required so that the field magnitude increases radially, i.e., a minimum-B configuration. This is because plasma tends to collect in regions of weaker field intensity. For multipoles of order $n$ the radial dependence is $r^{n-1}$ near the axis. At the midplane the axial field decreases in the radial direction. The combination of these two fields results in a minimum in the magnetic energy density somewhere between the axis and the wall.

Early on we decided to use an octupole, as opposed to a hexapole field, because a higher order multipole would provide higher field energy densities near the vacuum wall, and this seemed as high an order as was practical. Another reason was that this fit well with the pulser coil design. The pulser required eight sets of feedthroughs spaced azimuthally around the vacuum vessel. The octupole elements could fit in between the feedthroughs.

\subsubsection{Permanent Magnets}

In generating the multipole field it was realized that using permanent magnets was the simplest way to proceed. They are convenient and economical. For certain types of magnetic material and dimensions, effective pole currents of a few $\mathrm{kA}$ can be created. 
Another advantage is that these effective currents are very constant, contributing to quiescent plasma behavior.

Permanent magnets can create fields similar to multipole fields deriving from current elements, but there are differences. A current conductor produces a field that drops off inversely with distance from the conductor. In a permanent magnet multipole the conductor is replaced by a magnet which behaves as a dipole so that the field decreases as $1 / \mathrm{r}^{2}$. Nevertheless, Goede and Brakenhoff [9] demonstrated that permanent magnets can work quite effectively.

\subsubsection{Design and Construction of Permanent Magnet Octupole}

The magnet octupole consisted of eight rows of permanent magnets. Each row was made up of several bricks of magnetic ceramic material. The bricks were placed in an aluminum rack to hold them in place. Each rack was as long as the main chamber $(152 \mathrm{~cm}$, $60 \mathrm{in}$ ) and rested on the flanges of the vacuum sections. This put the magnets at a radius of $16.8 \mathrm{~cm}(6.625 \mathrm{in}$.). Each brick has the dimensions $15.2 \times 5.1 \times 1.3 \mathrm{~cm}(6 \times 2 \times 0.5 \mathrm{in}$.$) .$ The magnetization is along the short dimension. The bricks were laid in the rack in two levels, staggered, just as in a brick wall. This helped smooth out variations in the field produced by each brick. The magnet rows then had a cross-section of 2 in $\times 1$ in. The overall cross-section of the octupole is shown in fig. 3.5.

The field produced by the magnet bricks, as measured at the center of the broad face, varied from $350 \mathrm{~g}$ to $600 \mathrm{~g}$. The bricks were carefully arranged so that the average field of two bricks stacked together would be approximately the same everywhere.

\subsubsection{Calculation of $|\mathrm{B}(\mathbf{r})|$}

A calculation of the radial dependence of the magnitude of the $B$ field must be done to be certain that the minimum lies not too far from the axis. The following derivation is for field dependence along a radial line to the center of a magnet row. It can be deduced from 


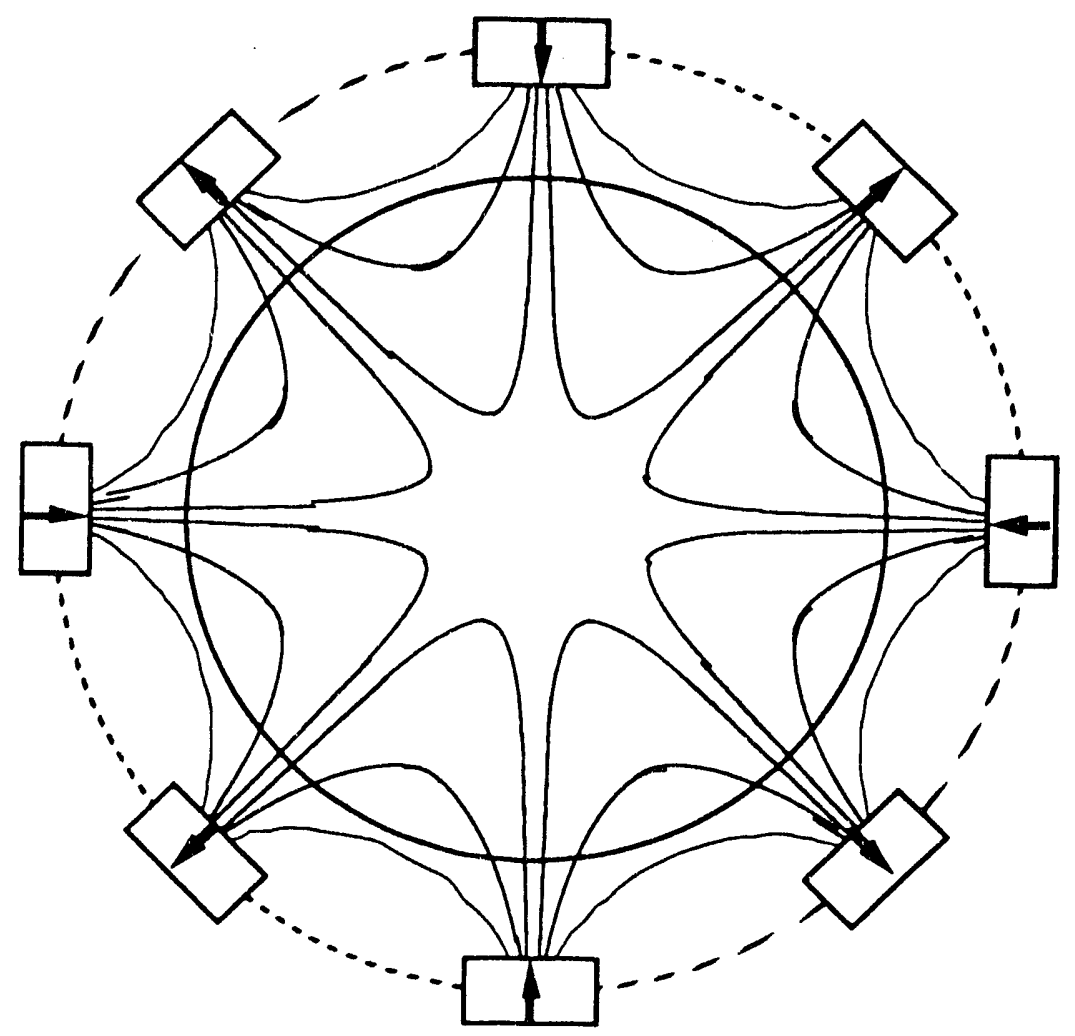

Fig. 3.5 This diagram shows the orientation of the octupole magnets along with some of the flux lines. The view is along the axis of the mirror field. The arrows show the $\mathbf{M}$ vector. The solid inner circle marks the inner wall.

symmetry that the transverse field along this line is purely radial. We can then proceed to calculate $B^{2}(r)=B_{z}^{2}(r)+B_{r}^{2}(r)$.

The radial behavior of the main axial field can be derived by approximating $B_{z}$ at $z=0$ (more accurately than what was done for the energy confinement calculation)

$$
\mathrm{B}_{\mathrm{z}}=\mathrm{B}_{\mathrm{o}}\left(1+\left(\frac{\mathrm{z}}{\mathrm{L}_{2}}\right)^{2}-\left(\frac{\mathrm{z}}{\mathrm{L}_{4}}\right)^{4}\right)
$$

where $L_{2}$ and $L_{4}$ are characteristic scale lengths. $A$ proper value for $L_{2}$ and $L_{4}$ as well as $B_{0}$ is determined by expanding the field from the electromagnets about $z=0$. Thus $L_{2}=93$ 
$\mathrm{cm}, \mathrm{L}_{4}=57 \mathrm{~cm}$, and $\mathrm{B}_{0}=700 \mathrm{~g}$. Then $\nabla \cdot \mathbf{B}=0$ is used to find $\mathrm{B}_{2}(\mathrm{r})$. This function is plotted in fig. 3.6.

$$
\mathrm{B}_{\mathrm{z}}(\mathrm{r})=\mathrm{B}_{\mathrm{O}}\left(1-\frac{1}{2}\left(\frac{\mathrm{r}}{\mathrm{L}_{2}}\right)^{2}-\frac{3}{8}\left(\frac{\mathrm{r}}{\mathrm{L}_{4}}\right)^{4}\right)
$$

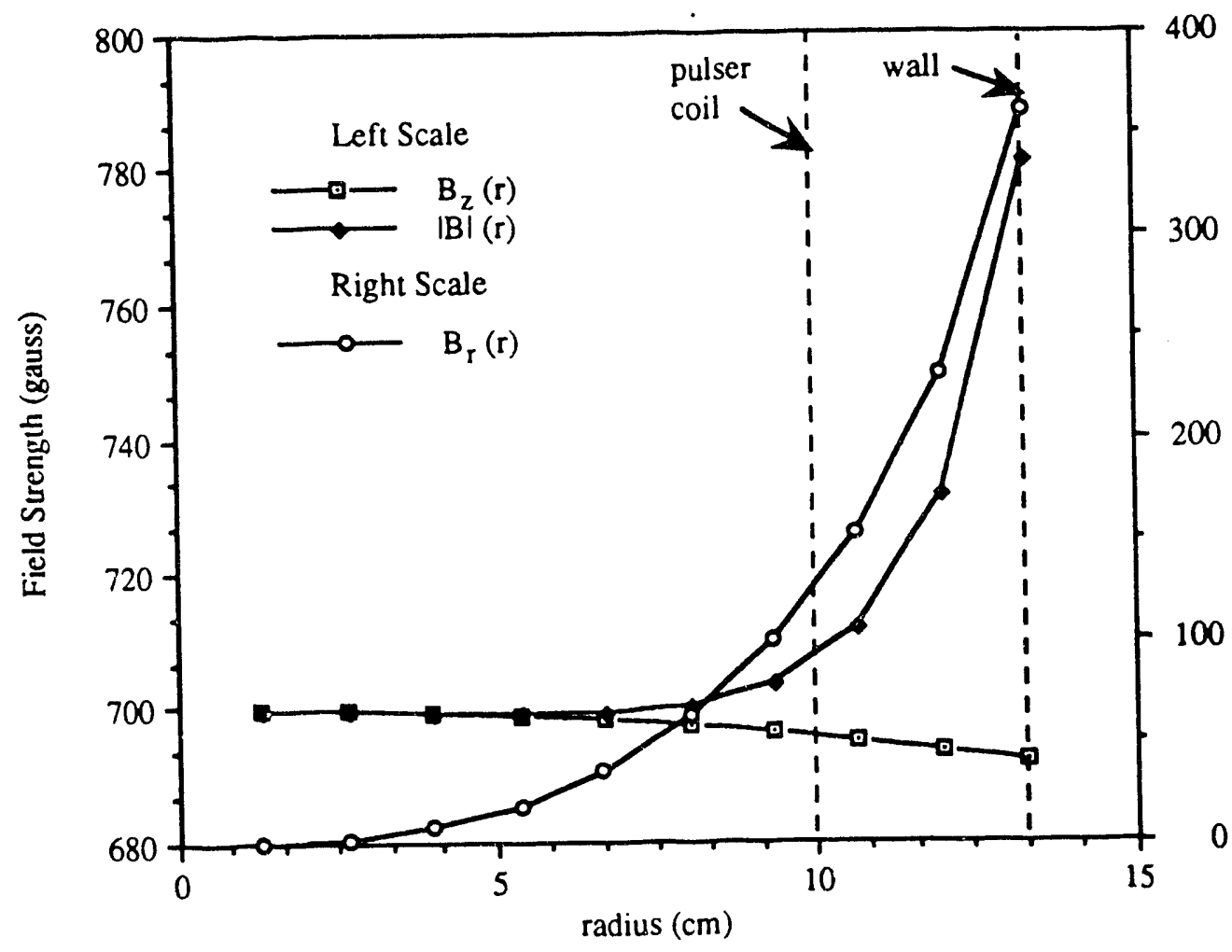

Fig. 3.6 Results of a calculation to find the radial position of the minimum in the $B$ field intensity. The minimum is at approximately $5.5 \mathrm{~cm}$.

To calculate $B_{r}(r)$ each magnet row is approximated by a pair of line currents spaced a distance equal to the magnet width. The line currents will be the effective magnetization current, $I_{m}$, flowing in the sides of the magnets. This current is $I_{m}=a J_{m}$, where a is the side dimension where the magnetization current density, $\mathrm{J}_{\mathrm{m}}$, flows. The current density, $\mathrm{J}_{\mathrm{m}}$, is determined by the magnetization vector $\mathbf{M}$, the magnitude of which is equivalent to 
$B_{r e m}$, the remnant $B$ field inside the magnet when there is no internal $H$ field. $B_{\text {rem }}$ is characteristic of the particular material that was used, Ceramic 8 . For this material $B_{\text {rem }}=$ 3900 gauss. Hence, the effective magnetization current is $I_{m}=7.9 \mathrm{kA}$.

Given $I_{m}$ the field of each magnet can be approximated by

$$
\begin{gathered}
\mathrm{B}_{\mathrm{r}^{\prime}}=\frac{\mu_{0} \mathrm{I}_{\mathrm{m}} \mathrm{w}}{\pi} \frac{\sin \phi^{\prime}}{\mathrm{r}^{\prime 2}} \\
\mathrm{~B}_{\phi^{\prime}}=\frac{\mu_{0} \mathrm{I}_{\mathrm{m}} \mathrm{w}}{\pi} \frac{\cos \phi^{\prime}}{\mathrm{r}^{\prime 2}}
\end{gathered}
$$

Here $w$ is the width of the magnet. The coordinates $r$ and $\phi^{\prime}$ are referenced from the center of the magnet. The magnetic induction along any radial line is now just a superposition of these fields. This calculation is most conveniently done by computer. Fig. 3.6 shows a calculation of $B_{r}(r)$ as well as $|B(r)|$ for a radial line from the axis to the center of a magnet. Values were only calculated for $\mathrm{r}<\mathrm{r}_{\text {wall }}$.

The result of the calculation shows that the B field minimum is at about $5.5 \mathrm{~cm}$. However, this is a very shallow minimum. For the most part we can say that the plasma profile will be of constant density out to $5.5 \mathrm{~cm}$, where it will begin to decrease to zero at the wall. When the pulser coil is installed the outer plasma boundary will be reduced to 10 $\mathrm{cm}$. However, the pulser coil will not seriously affect the plasma dimensions, since it is in a relatively high field region.

\subsection{EFFI Calculations and Plasma Dimensions}

More detailed calculations of the magnetic field can be done with the EFFI computer code. Current elements are specified as input to this program. The output gives the magnitude of the B field and its components as well as flux lines wherever requested. An advantage of EFFI is that the current can be represented more accurately as a uniform 
current density over the cross-section of the conductor. For the permanent magnets, the actual surface magnetization current density, $\mathrm{J}_{\mathrm{m}}$, can be given as input.

Fig. 3.4 shows the field magnitude along the axis. The peak field at the mirror is 1085 gauss. Hence the mirror ratio is 1.55 . Figs. 3.7 and 3.8 show flux lines that originate at the midplane on circles of $1.5(3.8 \mathrm{~cm}), 3(7.6 \mathrm{~cm})$, and $4(10.1 \mathrm{~cm})$ inches in radius. Also shown are mod-B contours. Fig. 3.9 shows the same flux lines (starting at a 3 in. radius) in the $\mathrm{r}-\mathrm{z}$ plane.

These field lines show well the curvature of the B field. However, they can also be used to find the plasma dimensions. To find the plasma density at a particular point in space, the distribution function, $f(v)$, is integrated at that point. A Maxwellian-loss-cone with different characteristic temperatures is assumed for $f(v)$.

$$
\begin{gathered}
f(v)=n_{0} \frac{m}{2 \pi T_{\perp}} \sqrt{\frac{m}{2 \pi T_{\|}}} \exp \left(-\frac{m}{2}\left(\frac{v_{\perp}^{2}}{T_{\perp}}+\frac{v_{\|}^{2}}{T_{\|}}\right)\right) \quad \theta>\theta_{m} \\
\tan \theta=\frac{v_{\perp}}{v_{\|}}
\end{gathered}
$$

For $\theta$ less than the loss-cone angle, $\theta_{m}, f(v)$ is zero. The local loss-cone angle depends on the mirror ratio at that point, $\sin \theta_{m}=1 / \sqrt{R_{m}}$. To find $R_{m}$, the field line that passes through that point is considered. The ratio of the maximum $B$ along the field line to $B$ at that point is $R_{m}$. If the field line strikes the wall, then $B$ at the wall defines $R_{m}$. Gyroradius effects are neglected here. 


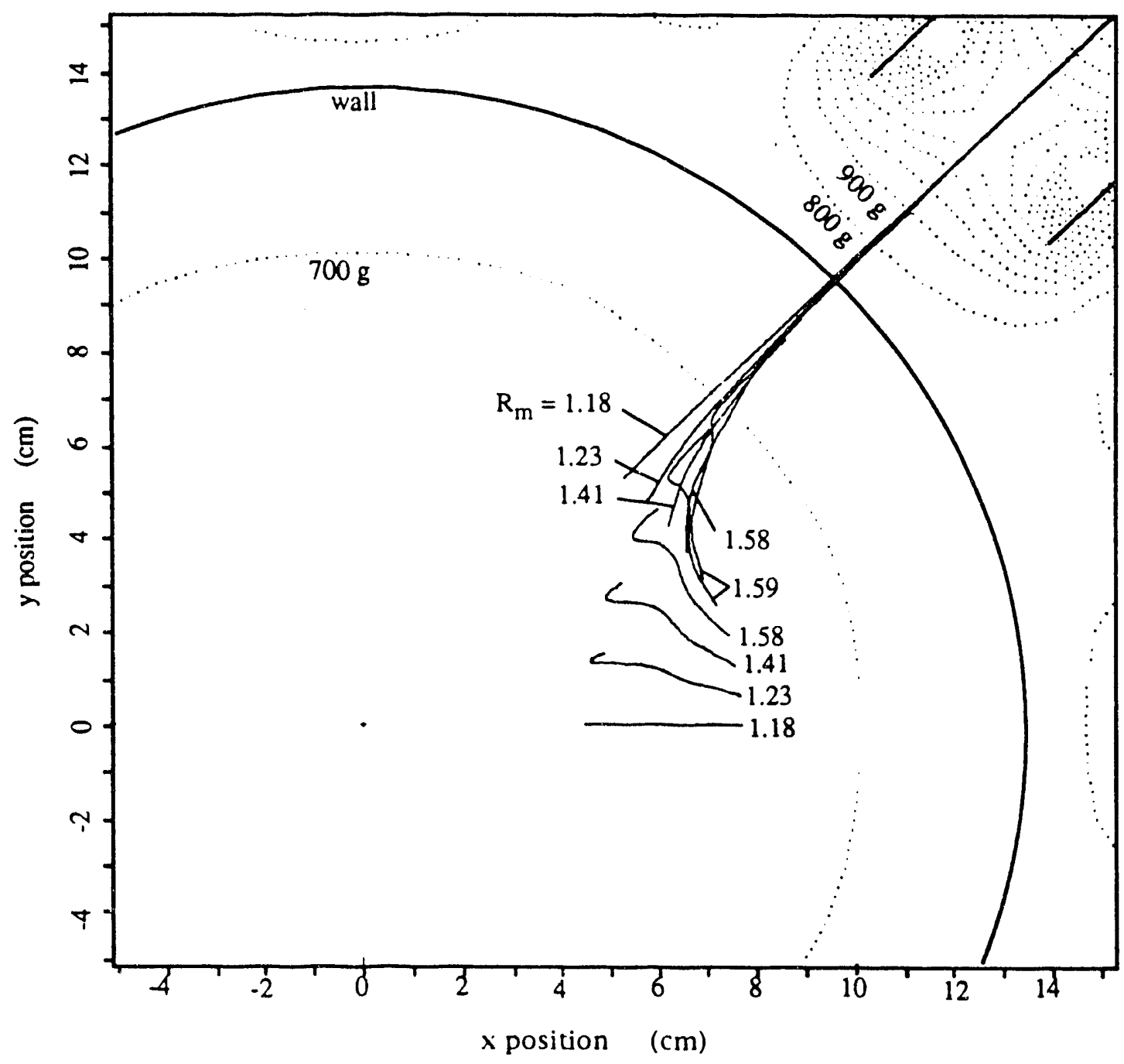

Fig. 3.7 An EFFI plot of field lines originating at the midplane on a $45^{\circ}$ arc of $7.6 \mathrm{~cm}$ ( 3 in.) radius. The mirror ratio for each field line is shown. Plots such as this help to determine the plasma dimensions. 


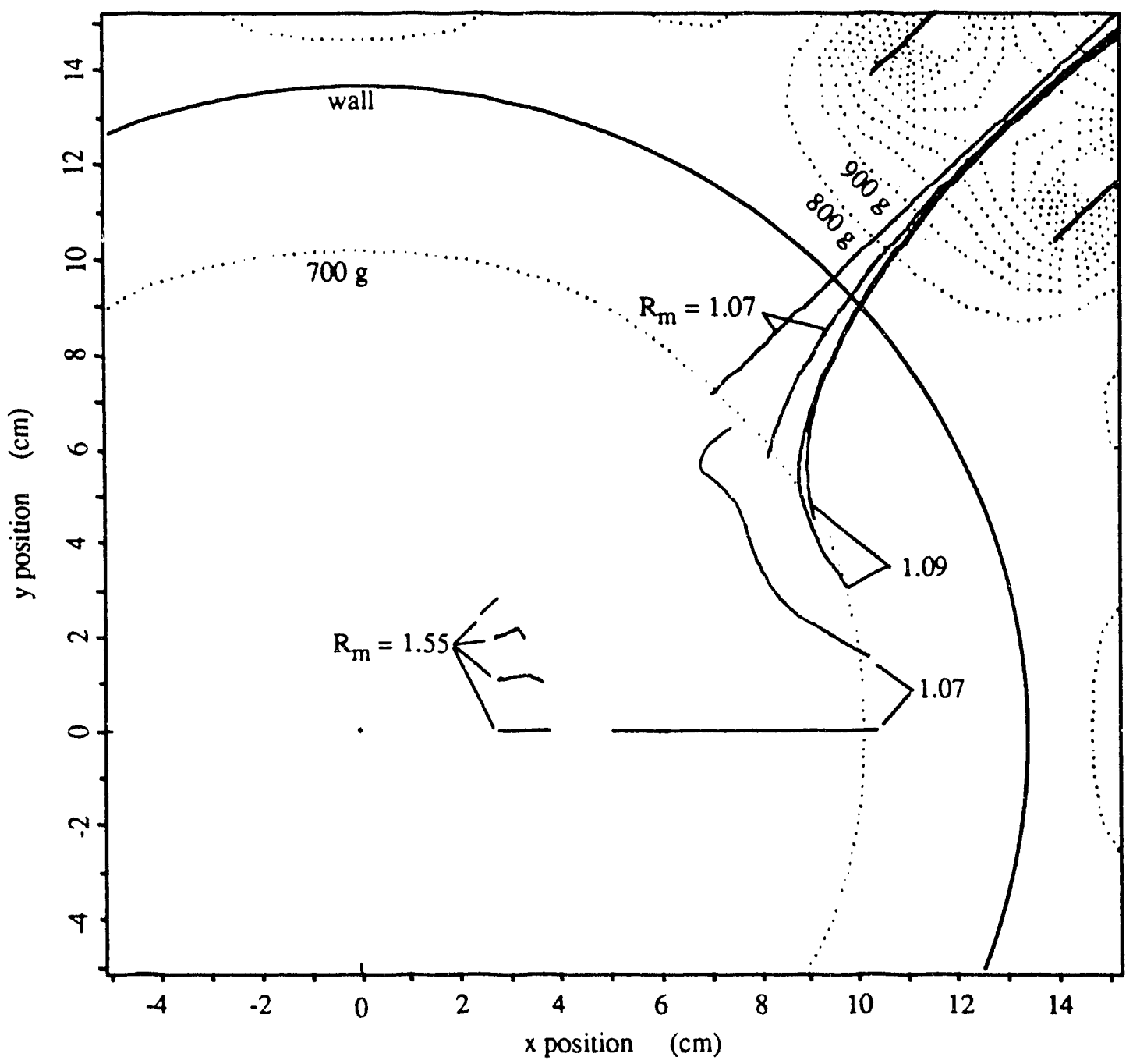

Fig. 3.8 An EFFI plot of field lines originating at the midplane on a $45^{\circ}$ arc of 3.8 and $10.1 \mathrm{~cm}(1.5,4 \mathrm{in}$.) radius. The mirror ratio along each line is given. Plots such as this help to determine the plasma dimensions. 


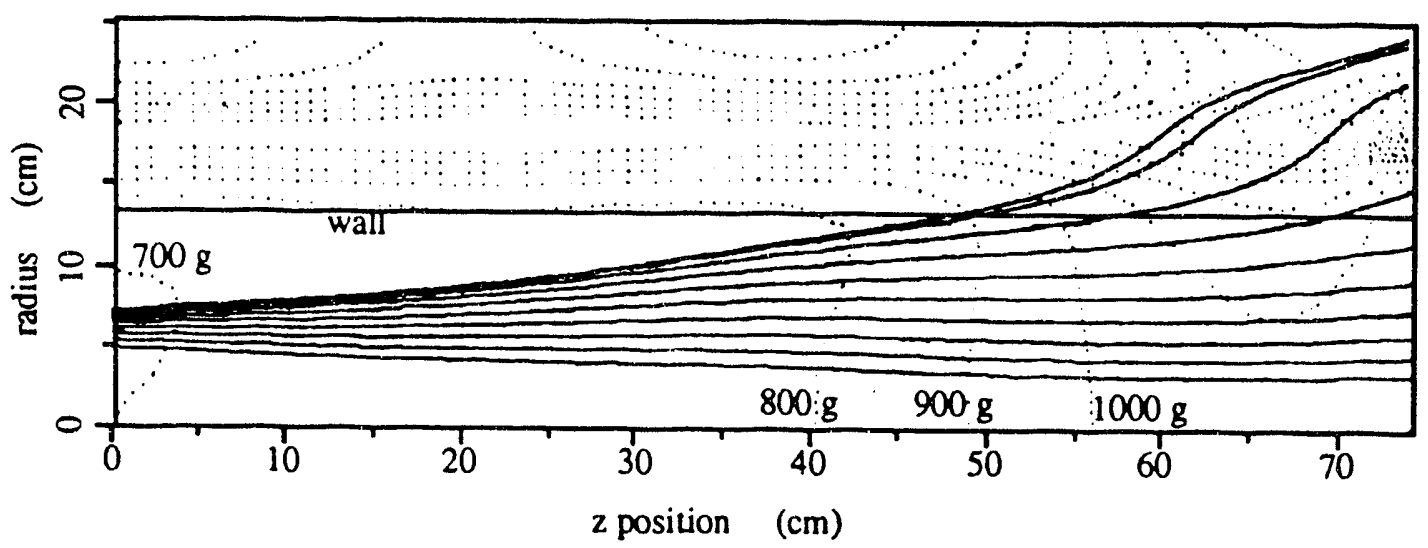

Fig. 3.9 An EFFI plot of field lines originating on a $45^{\circ}$ arc of a $7.6 \mathrm{~cm}(3$ in.) circle. The azimuthal plane is oriented in the direction of a magnet with magnetization pointed radially outward. Hence the top most field line is in the plane of the plot. The others are projected onto the plane of the plot.

Integrating $f(v)$ over $v$ gives

$$
n(r)=\frac{n_{Q}}{\sqrt{1+\alpha \tan ^{2} \theta_{m}}}
$$

where $n_{0}$ is just the density in a uniform Maxwellian plasma and $\alpha=T_{\mathbb{V}} / T_{\perp}$. The spatial dependence is contained in $\theta_{\mathrm{m}}$. It is expected that the density will be greatest at the midplane, on axis, so the local density should be normalized to this. The loss-cone angle here is $\theta_{m o}$ and the mirror ratio is $R_{m o}=1.55$. The center density is $n\left(\theta_{m o}\right)$. The normalized density is $\underline{\underline{n}}=\mathrm{n}\left(\theta_{\mathrm{m}}\right) / \mathrm{n}\left(\theta_{\mathrm{mo}}\right)$.

$$
\begin{aligned}
\underline{\mathrm{n}} & =\sqrt{\frac{1+\alpha \tan ^{2} \theta_{\mathrm{mo}}}{1+\alpha \tan ^{2} \theta_{\mathrm{m}}}} \\
& =\sqrt{\frac{1+\alpha /\left(\mathrm{R}_{\mathrm{mo}}-1\right)}{1+\alpha /\left(\mathrm{R}_{\mathrm{m}}-1\right)}}
\end{aligned}
$$


The spatial dependence is now in $R_{m}=B_{\max } / B(r)$, where $B_{\max }$ is the maximum $B$ along a field line that passes through the point defined by $\mathbf{r}$.

Now by looking at $B(z)$ on axis in fig $3.4, R_{m}(z)$ and hence $n(z)$ can be calculated.

Fig. 3.10 shows the density along the axis for different temperature ratios. The plasma can be very uniform out to the mirrors especially for electrons with high perpendicular energy. This is the kind of distribution that is typical for an ECRH plasma.

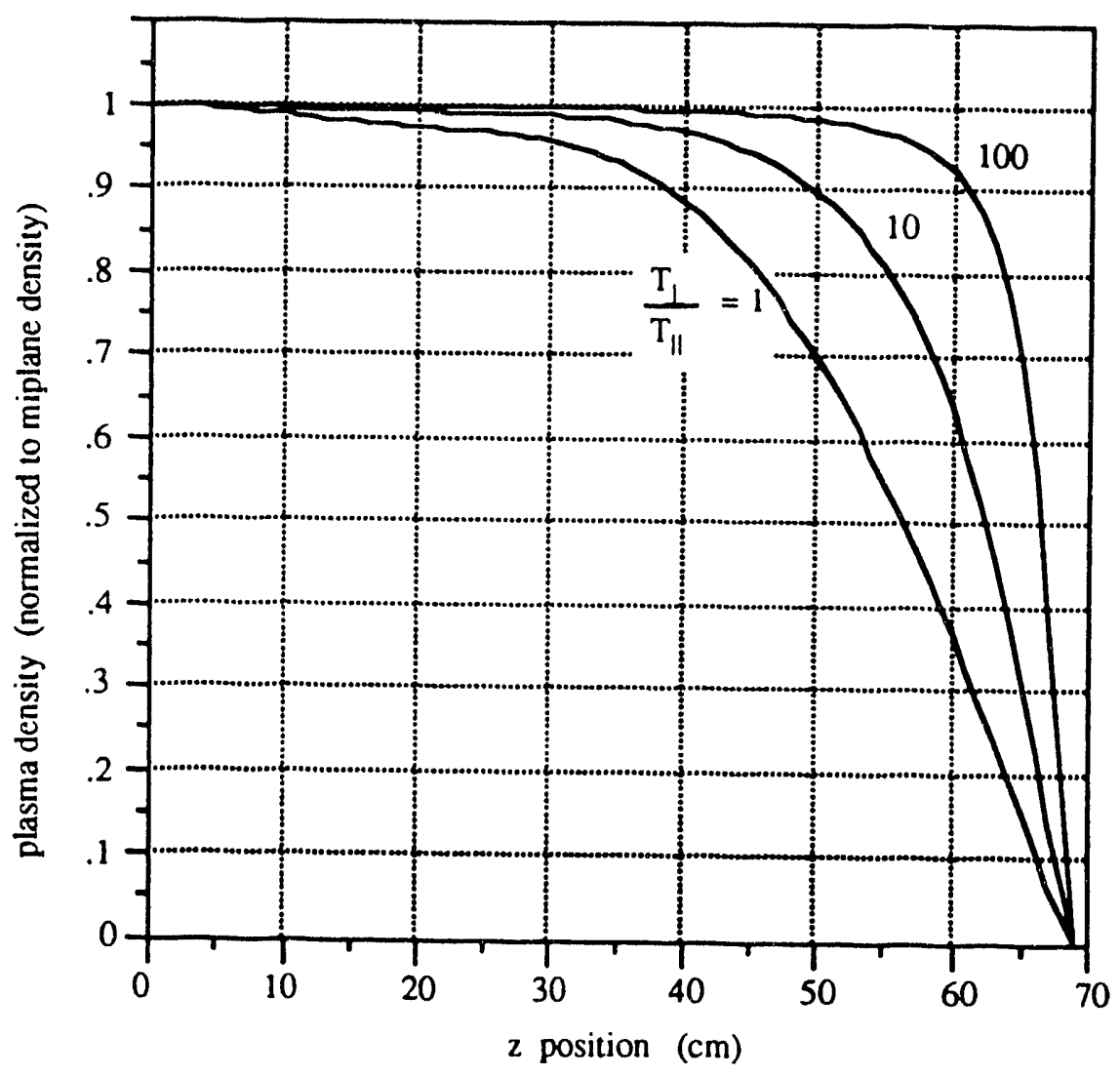

Fig. 3.10 Normalized plasma density along the axis. Density on axis is calculated from field line analysis. The higher the perpendicular temperature is, the more uniform the plasma column.

Radial density profiles can be obtained the same way by analyzing the field lines in figs. 3.7 and 3.8. The local mirror ratios in the midplane are sampled at these field line locations, and the density profile is calculated in fig. 3.11. 
In an azimuthal direction between the magnets the density peaks off axis just as expected from the minimum-B calculation. The density in a direction azimuthally aligned with the magnets drops off more quickly. This is because the field lines here swing out to the walls quickly so that only deeply trapped electrons remain.

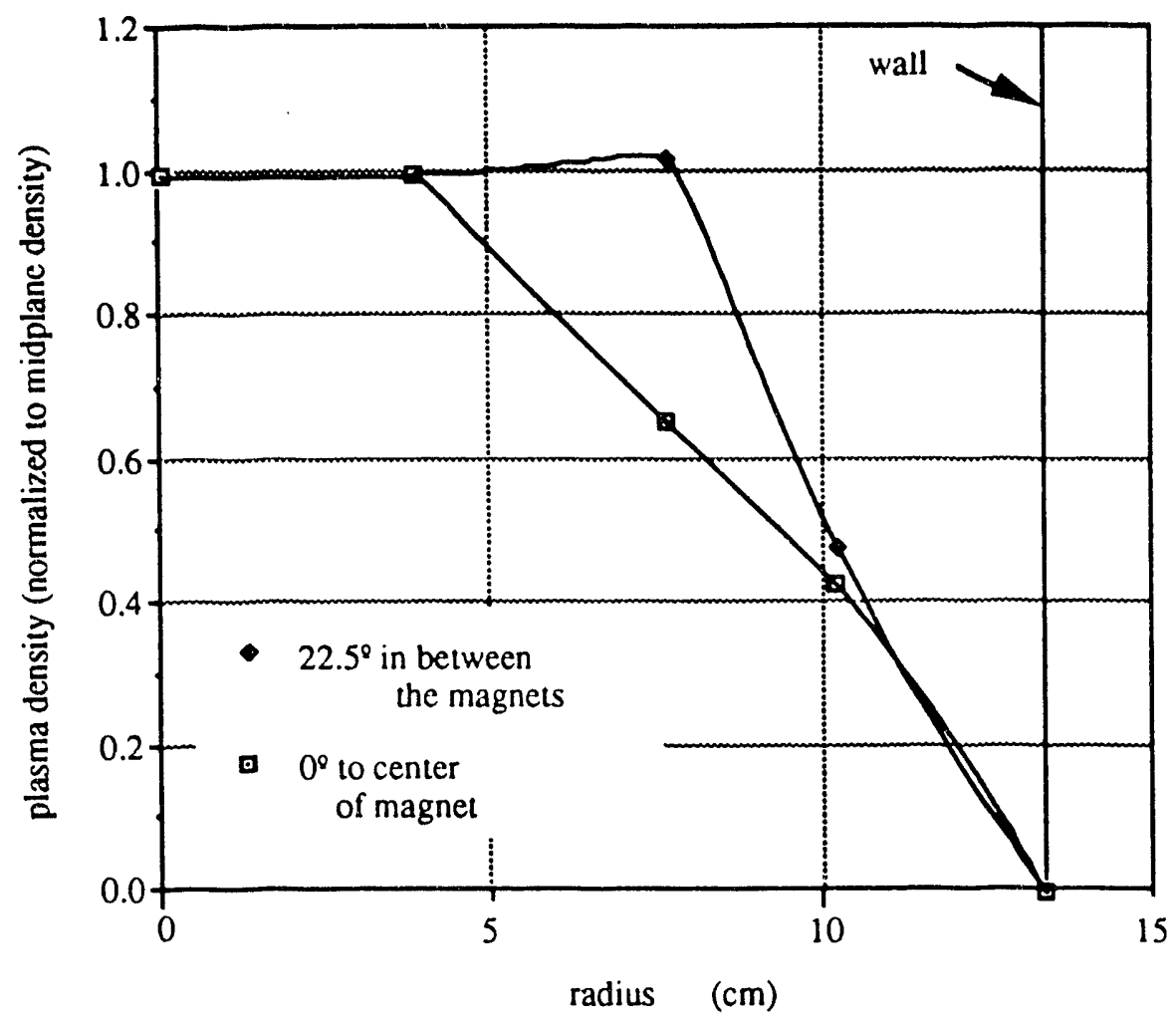

Fig. 3.11 Normalized radial plasma density profile derived from EFFI field calculations. This plot is for equal temperatures in the perpendicular and parallel directions, and for $z=0$.

\subsection{ECRH}

Theory shows that the expected potential is proportional to the electron temperature. A hot electron plasma is easily obtained with electron cyclotron heating. To increase the efficiency of our ECRH system, two sources were used, each with a different frequency. Further efficiency is achieved when each source is coupled to resonant cavity modes of the vacuum chamber. 


\subsubsection{Two Frequency Heating}

\subsubsection{Theory}

In this method of heating the first frequency couples to the electron cyclotron resonance. The gas is broken down, and the electrons are heated to good confinement. The second frequency can then transfer significant power to the plasma by second harmonic heating. Second harmonic heating means heating at a frequency $\omega=2 \omega_{c}$. Even then this only works if the electric field is non uniform over the path of the cyclotron orbit.

To demonstrate, consider a linear polarized electric field oscillating at frequency $\omega$, perpendicular to the $\mathbf{B}$ field, $\mathbf{E}_{\perp}=\mathrm{E}_{\mathrm{O} \perp} \cos (\omega t) \mathbf{x}$. The velocity of an electron in a magnetic field is $\mathbf{v}_{\perp}=v_{0 \perp}\left(\cos \left(\omega_{c} t\right) \mathbf{x}+\sin \left(\omega_{c} t\right) \mathbf{y}\right)$. The work done on the electron over one period is (using the unperturbed electron orbit)

$$
\begin{aligned}
W & =\int_{0}^{t} e E_{\perp} \cdot v_{\perp} d t^{\prime} \\
& =e E_{0 \perp} v_{0 \perp} \int_{0}^{t} \cos \left(\omega t^{\prime}\right) \cos \left(\omega_{c} t^{\prime}\right) d t^{\prime} \\
& =e E_{0 \perp} v_{0 \perp}\left(\frac{\sin \left(\omega_{c}-\omega\right) t}{2\left(\omega_{c}-\omega\right)}+\frac{\sin \left(\omega_{c}+\omega\right) t}{2\left(\omega_{c}+\omega\right)}\right)
\end{aligned}
$$

The second term is a fast oscillating term that does not contribute net energy to the electron. The same is true of the first term except in the limit as $\omega \rightarrow \omega_{c}$, in which case we have $\mathrm{W}=\mathrm{eE}_{\mathrm{O} \perp} \mathrm{v}_{\mathrm{O} \perp} \mathrm{V}$. This is just the fundamental cyclotron resonance. In the resonance case the velocity increases linearly in time so that $W$ actually increases as $t^{2}$.

Now consider an $\mathbf{E}$ field with a spatial gradient. In the reference frame of the electron it will appear that the magnitude of the electric field is varying with a frequency $\omega_{\mathrm{c}}$. Assume now that $E_{O \perp}$ becomes $E_{O \perp}\left(1-\frac{\varepsilon}{2}\left(1-\cos \left(\omega_{c} t\right)\right)\right.$ where $\varepsilon$ is the fractional variation of the $\mathbf{E}$ field over the diameter of the orbit. The work done over an orbit is then 


$$
\mathrm{W}=\mathrm{eE}_{0 \perp} \mathrm{v}_{0 \perp}\left(\left(1-\frac{\varepsilon}{2}\right) \int_{0}^{t} \cos \left(\omega t^{\prime}\right) \cos \left(\omega_{c} t^{\prime}\right) d t^{\prime}+\frac{\varepsilon}{2} \int_{0}^{t} \cos \left(\omega t^{\prime}\right) \cos ^{2}\left(\omega_{c} t^{\prime}\right) d t^{\prime}\right)
$$

The first term is just like the case discussed above. In the case of off-resonant heating, $\omega \neq$ $\omega_{c}$, and the first term contributes no net energy. Integrating the second term gives

$$
\mathrm{W}=e E_{0 \perp} v_{0 \perp} \frac{\varepsilon}{2}\left(\frac{\sin (\omega t)}{2 \omega}+\frac{\sin \left(2 \omega_{c}-\omega\right) t}{4\left(2 \omega_{c}-\omega\right)}+\frac{\sin \left(2 \omega_{c}+\omega\right) t}{4\left(2 \omega_{c}+\omega\right)}\right)
$$

In this case, the time averaged work done is zero unless $\omega=2 \omega_{\mathrm{c}}$. The middle term will contribute to give $\mathrm{W}=\mathrm{eE}_{\mathrm{O} \perp} \mathrm{v}_{\mathrm{O} \perp} \varepsilon \mathrm{E} / \mathrm{8}$. This shows that energy transfer to the electron at the second cyclotron harmonic is possible. However, it requires that the gyroradius be comparable to a scale length for electric field change.

For standing waves in a cavity this scale length might be the distance between lodes of the field. This distance is shorter for field configurations with higher mode numbers, i.e., higher frequencies. For example, if there are two radial nodes then the distance between them is $R_{\text {wall }} / 3=4.4 \mathrm{~cm}$ and the distance from peak field to node is $2.2 \mathrm{~cm}$. If the fundamental frequency can heat an electron to $10 \mathrm{keV}$ in a $700 \mathrm{~g}$ magnetic field, then its gyroradius is $r_{g}=0.48 \mathrm{~cm}$. This example indicates about a $20 \%$ variation in the electric field over the electron path, which can lead to considerable energy gain over many revolutions.

\subsubsection{ELMO Experiment at ORNL}

Dandl, et. al. [5] realized the effectiveness of non-resonant heating during experiments on high beta plasmas in simple mirror traps. They were heating a plasma with microwave fower ai $35.7 \mathrm{GHz}$. The resonance zone was near the mirror throat. They realized that relativistic electrons were still absorbing microwave power even though their mass shift 
made resonance at their gyrofrequency impossible. This led to experiments where a second independen 1 aicrowave source at a higher frequency of $55 \mathrm{GHz}$ was used to heat the plasma. The electron temperature increased only slightly, but the density increased by a factor of six for equal amounts of low and high frequency power.

\subsubsection{Microwave Sources}

\subsubsection{Origin}

For our experiment we obtained two continuous microwave sources. They were Air Force surplus, and used for electronic countermeasures. Each sol je contained a tunable, noise modulated magnetron before modifications. One magnetron is tunable from $2 \mathrm{GHz}$ to $3 \mathrm{GHz}$, and the other has a frequency range of $3.6 \mathrm{jHz}$ to $5 \mathrm{GHz}$. Each is rated at 300 watts output into a matched load.

Each : urce came with its own power supply to provide $-3 \mathrm{kV}$ to the magnetron filament. These supplies converted $400 \mathrm{~Hz}$ aircraft power. Therefore, a motor-generator was provided with the system to turn $60 \mathrm{~Hz}$ power to $400 \mathrm{~Hz}$.

\subsubsection{Modifications}

These sources were modified for our use. A steady narrow band frequency with constant output was desired. Hence the noise modulation tube was removed. Additional chokes and capacitors were added to the high voltage bias to smooth out the $400 \mathrm{~Hz}$ voltage fluctuations. Tuning was done by means of hydraulically controlled pumps. This was replaced by a mechanical tuning system for better responsiveness.

The control system was not provided with the sources. Therefore, a control unit was made to vary the output power of the magnetrons. This was accomplished by feeding the $400 \mathrm{~Hz}$ power through a variac, reducing the input power to the magnetron high voltage supply. This in turn reduced the $-3 \mathrm{kV}$, bias voltage on the magnetron cathode. This lowered the intensity of the microwaves from the magnetron. 
Because the microwaves were not being transferred to a matched load, there was certain to be considerable reflected power. Since the magnetron tubes were not designed with this in mind, isolators were placed in the cable line to the antennae. Reflected power was attenuated to a less than one watt.

\subsubsection{Antennae}

Because of the relatively low power of the sources, it was decided to increase the efficiency of heating by coupling the microwave power to cavity resonances of the vacuum chamber. The shape of the antenna and the way it is inserted into the cavity strongly excites the $\mathrm{z}$ component of the cavity electric field.

The power from the magnetron is coupled out by means of waveguide which is then adapted to RG 213/U transmission cable. The end of the cable had the shielding stripped back so that the center conductor and the surrounding dielectric were exposed. Thus, the microwaves radiate from the end of the cable like an antenna.

The radiating end of the coax cable is then inserted into ports on the end of the vacuum chamber. These ports are shown in fig. 3.2 and fig. 3.12. A hole was drilled into the end flange and a capped ceramic tube was inserted and brazed into place. There are four ports placed $90^{\circ}$ apart at a radius of $10.1 \mathrm{~cm}(4$ in.). They can be identified by their clock positions. The 12 and $60^{\prime}$. lock ports have a $.7 \mathrm{~cm}$ inner diameter and the 3 and 9 o'clock ports have a $1.1 \mathrm{~cm}$ inner diameter. It is not certain what effect the port diameter has on coupling, but two different sizes would allow a choice. It can then be experimentally determined which size is better for microwave coupling

When the antenna is inserted into this tube, the radiating electric field will extend through the ceramic to the inside surface of the end flange. This electric field will match the electric field of the cavity that radiate normally from the end flange. Hence, the microwaves will couple strongly to any cavity standing waves that have a $\mathrm{z}$ component. 
It is difficult to say that coupling strictly depends on $E_{z}$, because of the other field components from the antenna. However, coupling can be maximized for each mode coupled to by varying how far the antenna is inserted into the port. The best position will be determined experimentally by measuring the reflected power and minimizing it.

\subsubsection{Cavity Resonances}

It was decided early on to have a vacuum chamber as close to a perfect cylinder as possible. The main reason was to maximize the $Q$ factor of the cavity. As such, the $Q$ was measured to be about 8000 . Also, calculating the resonant modes and their frequencies would then be a simple task. Only the transverse magnetic modes are important since the antenna configuration will strongly excite an $E_{z}$ component that only the TM modes have.

\subsubsection{Mode Calculations}

It is important to know exactly which modes are being coupled to because only certain modes are desired. The modes to be coupled to can be determined from the frequency of the mode. The frequency of a particular mode is given by

$$
f_{m n p}=\frac{c}{2 \pi \tilde{n}} \sqrt{\left(\chi_{m n} / R\right)^{2}+(p \pi / d)^{2}}
$$

The mode numbers $\mathrm{m}, \mathrm{n}$, and $\mathrm{p}$ describe the mode configuration and its oscillation frequency. Here $c$ is the speed of light, $\tilde{n}$ is the index of refraction of the medium in the vacuum vessel, $\chi_{m n}$ is the $n$th zero of the $m$ th order Bessel function, $R$ is the cylinder radius, and $d$ is the length of the cylinder. The electric field components of the corresponding mode are

$$
E_{r}=-E_{o} \frac{p \pi}{d} \frac{R}{\chi_{m n}} J_{m}^{\prime}\left(\frac{\chi_{m n} r}{R}\right) \cos (m \theta) \sin \left(\frac{p \pi z}{d}\right)
$$




$$
\begin{gathered}
E_{\theta}=E_{o} \frac{m}{r} \frac{p \pi}{d}\left(\frac{R}{\chi_{m n}}\right)^{2} J_{m}\left(\frac{x_{m n} r}{R}\right) \sin (m \theta) \sin \left(\frac{p \pi z}{d}\right) \\
E_{z}=E_{o} J_{m}\left(\frac{x_{m r^{r}}}{R}\right) \cos (m \theta) \cos \left(\frac{p \pi z}{d}\right)
\end{gathered}
$$

\subsubsection{Mode Selection}

Several factors determine which mode should be excited. First, we would like to couple to just one mode to maximize the field strength. This means that we want a frequency that is relatively isolated from other mode frequencies. Second, the desired mode should have a strong field on axis that is transverse to the magnetic field, i.e., $r$ and $\theta$ components. This component will be the one that ionizes and heats the plasma. Ideally the plasma should be generated near the axis where the neutral gas is highly concentrated. The only $\mathrm{TM}$ resonances that have non-vanishing electric field components at $\mathrm{r}=0$ are the $\mathrm{m}=$ 1 modes.

The $\mathrm{TM}_{1,1,20}$ mode was chosen for fundamental heating at $2.37 \mathrm{GHz}$. The radial electric field on axis for this mode is $240 \mathrm{~V} / \mathrm{cm}$ if 100 watts of average power is directed into the cavity. This calculation is based on the measured $\mathrm{Q}$ factor. Cavity calculations show that it is isolated by several MHz.

The second heating frequency must be greater than $3.92 \mathrm{GHz}$, if the midplane field is $700 \mathrm{~g}$. The actual frequency chosen for the first tests was the $\mathrm{TM}_{1,2,31}$ mode at 3.912 $\mathrm{GHz}$. This choice was $\mathrm{n}$ ¿de based on this mode's frequency isolation without paying attention to the minimum value of $2 \omega_{c}$ in the cavity. The secondary heating was still accomplished, however, because the first power supplies produced only enough current to generate about $600 \mathrm{~g}$ at the midplane, thus allowing smaller heating frequencies.

\subsubsection{Measurement of Cavity Modes}

Some of the first experimental work done on the Mirrortron was measuring the resonances of the cavity. Measuring the resonances could be done in two ways. One was to 
insert a second antenna-cable into one of the other ports to sample the resonant power in the cavity. Due to the uncertainty over whether the second antenna was detecting cavity fields or direct radiation from the power antenna, the method of reflected power was preferred. This involved placing a directional coupler in line between the source and the antenna. In either case a bolometer or a crystal detector was used to measure the signal.

To probe the cavity an HP8341A frequency synthesizer was used. A narrow bandwidth and constant output over a wide frequency range allowed precise measurements of the resonances. Typically a bolometer was used with an HP432A power meter. Data was recorded on an X-Y recorder. A 0-1 volt full scale output from the power meter went to the $\mathrm{Y}$ input. A $0-10$ volt signal from the synthesizer that corresponded to a programmed frequency range went to the $\mathrm{X}$ input. In this way resonance plots were made.

By using the port's azimuthal positions it is possible to determine whether the mode number is odd or even, since the $E_{z}$ component varies as $\cos (m \theta)$. Fig. 3.12 shows the ports in reference to the azimuthal intensity pattern. If the input antenna is at 9 o'clock $(\theta=$ 0 ) then a second antenna at 12 or $6 o^{\prime} \operatorname{clock}(\theta= \pm \pi / 2)$ should measure zero if $m=o d d$ and non-zero if $m=$ even. It turns out that this method is complicated by the fact that the ports are of different sizes. The smaller ports measure weaker signals. Hence, it is unclear whether a small bump is a result of coupling to non $E_{z}$ components of an $m=$ odd mode, or if it is an attenuated non-zero measurement of an $\mathrm{m}=$ even mode.

It was decided to measure the fundamental TM modes first because it would be easy to identify them and because they could be used to determine an effective $R$ and $d$. These values might be modified because the cavity will not be a perfect cylinder. The actual values for $\mathrm{R}$ and $\mathrm{d}$ are $13.335 \mathrm{~cm}$ and $152.40 \mathrm{~cm}$, respectively. Also these modes can be used later for easy comparison of frequency shift after the pulser coil is installed. 


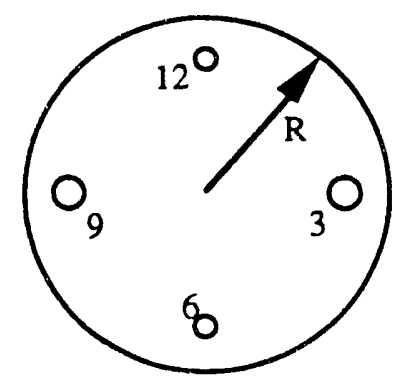

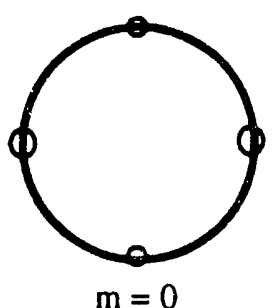

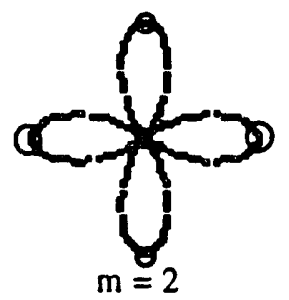

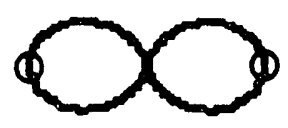

$\stackrel{0}{m=1}$

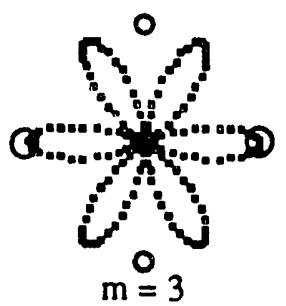

Fig. 3.12 Antenna port locations on the end flange. Also shown are the azimuthal field energy density patterns for different m modes. The microwave input is at 3 or 9 o'clock.

Fig. 3.13 shows the lowest TM modes, the $m=0, n=1, p=0,1,2 \ldots$ series. Table 3.4 lists the first three measured modes and these modes calculated from the actual value for $\mathrm{R}$ and $\mathrm{d}$. TE modes have lower frequencies but they are evidently not excited. By applying a least squares fit of the first 10 resonances to eq. 3.15 , effective values of $R$ and $\mathrm{d}$ were determined to be $13.339 \pm 0.005 \mathrm{~cm}$ and $153.29 \pm 0.1 \mathrm{~cm}$. The effective $\mathrm{R}$ matches its physical value, but the cylinder length is effectively longer, due mostly to the modifications at both ends of the cavity for gas flow and pumping.

Table 3.4

$\mathrm{TM}_{0,1}$ Modes

\begin{tabular}{||c|c|c||}
\hline \multicolumn{1}{c|}{$p$} & $\mathrm{f}$, meas. $(\mathrm{MHz})$ & $\mathrm{f}, \mathrm{calc} .(\mathrm{MHz})$ \\
\hline 0 & 860.4 & 861.0 \\
1 & 866.6 & 866.6 \\
2 & 883.1 & 883.3
\end{tabular}




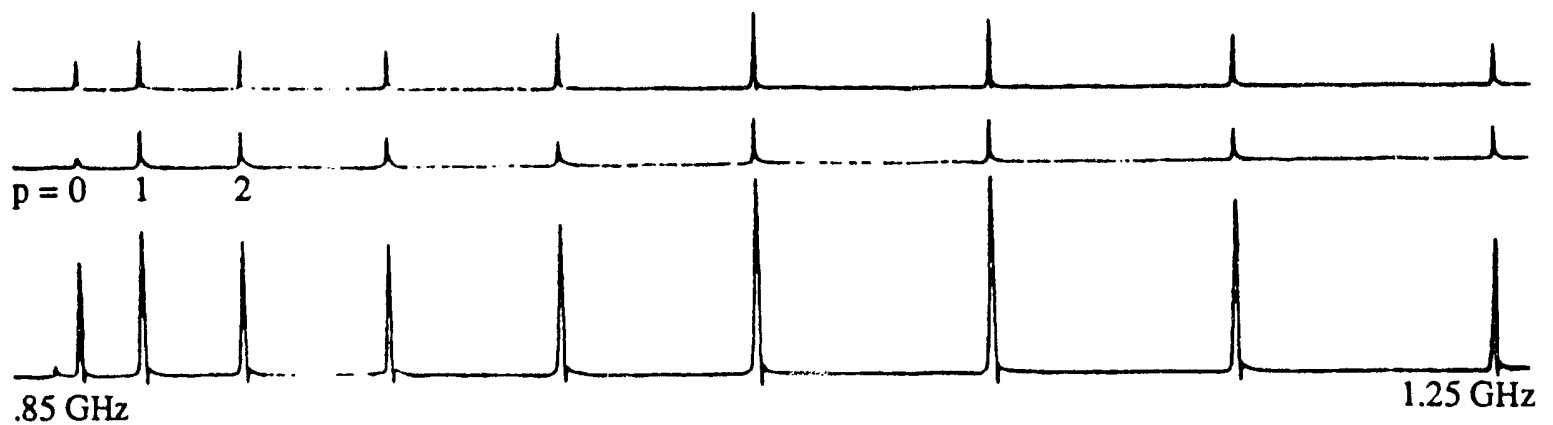

Fig. 3.13 The first TM resonances of the cavity. Microwaves were launched from the 9 o'clock port. The bottom trace is the power signal detected at the 3 o'clock port. The top two traces are the 6 and 12 o'clock ports. The smaller port size means a smaller detected signal.

Next, frequencies in the fundamental heating range were analyzed. The $m=1, n=1$ series begins at about $1.36 \mathrm{GHz}$. By doing another least squares fit on the $\mathrm{TM}_{1,1}$ series using the first 14 resonances, slightly different values for $R$ and $d$ were found; $R=13.402$ $\pm .006 \mathrm{~cm}, \mathrm{~d}=153.97 \pm .13 \mathrm{~cm}$. Using these values to calculate the rest of the $\mathrm{TM}_{1,1}$ series will give more accurate frequencies. Table 3.5 gives a list of calculated frequencies from 2.3 to $2.4 \mathrm{GHz}$. In fig. 3.14 , a resonance plot in this same range is made.

The $\mathrm{TM}_{1,1,20}$ mode is calculated to be $2.3774 \mathrm{GHz}$. In the resonance plots this seems to match best to the peak at $2.374 \mathrm{GHz}$. The calculated $\mathrm{TM}_{3,1,6}$ and $\mathrm{TM}_{3,1,7}$ modes do not appear to be excited at all. Hence the $\mathrm{TM}_{1,1,20}$ mode is even better isolated. The width of the magnetron output is about 20 to $30 \mathrm{MHz}$. Hence, the microwaves will couple to this one mode quite well. 
Table 3.5

TM Modes Between 2.3 and $2.4 \mathrm{GHz}$

\begin{tabular}{|c|c|c|c||}
\hline $\mathrm{m}$ & $\mathrm{n}$ & $\mathrm{p}$ & freq $(\mathrm{GHz})$ \\
3 & 1 & 4 & 2.30458 \\
0 & 1 & 22 & 2.30657 \\
3 & 1 & 5 & 2.32302 \\
0 & 2 & 13 & 2.33750 \\
2 & 1 & 15 & 2.33996 \\
3 & 1 & 6 & 2.34535 \\
3 & 1 & 7 & 2.37147 \\
1 & 1 & 20 & 2.37740 \\
0 & 2 & 14 & 2.39161 \\
0 & 1 & 23 & 2.39724
\end{tabular}

The next step was to find an optimum insertion point. Looking at reflected power and cavity power using a second antenna yielded similar results. Fig. 3.15 shows several traces of reflected power corresponding to different insertion depths. The frequency shift and decrease in $\mathrm{Q}$ is evident as the antenna is pushed deeper into the cavity. The top trace is different from the others because the tip of the antenna is tourhing the metal end cap of the ceramic tube, thus creating a completely different radiation pattern. Also the waving baseline output from the directional coupler is somehow dependent on this radiation pattern. Hence, this technique is difficult to use for direct comparisons of absolute reflected power. This aside, the best position is shown to be $1.6 \mathrm{~cm}$ from maximum insertion.

Fig. 3.16 shows a mode plot in the vicinity of the second harmonic heating frequency. The first thing that one notices is that the mode density is higher. The different port sizes make mode identification for the second frequency a little more difficult. There does seem to be a pair of strong modes at $3.915 \mathrm{GHz}$. 


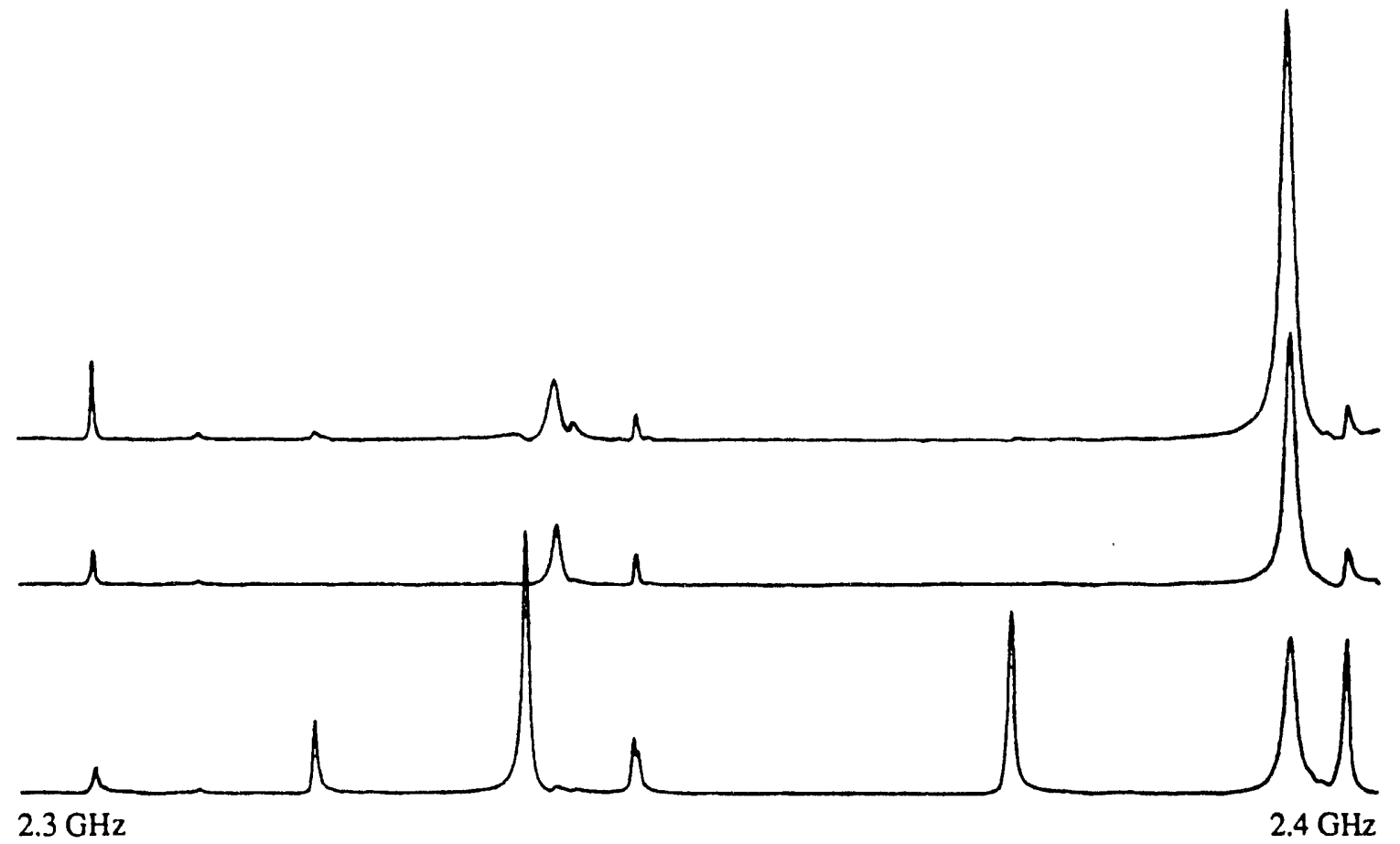

Fig. 3.14 A resonance plot of the cavity containing the $T M_{1,1,20}$ mode at $2.374 \mathrm{GHz}$. Input is at 9 o'clock. The bottom trace is the signal at 3 . The top two are the signal at 6 and 12. Even m modes have peaks in all three traces. Odd $\mathrm{m}$ modes only have peaks on the bottom trace.

It may not be of importance which mode is coupled to. For one thing the bandwidth of the second source is wider, several tens of $\mathrm{MHz}$. This guarantees that it will not be possible to couple to just one frequency alone. One mode excitation is desirable from the standpoint of having a maximum electric field strength inside the cavity. However, what is more important is maximum average power absorption. Coupling to several modes may be more efficient because of a more uniform distribution of heating zones throughout the plasma.

In any event, probably the best way to find an efficient heating frequency that works is to create a plasma using the low frequency source, then scan the high frequency source and look at the $x$-ray output of the plasma. Maximized output indicates the most efficient electron heating. 
Antenna position in the port can be optimized the same way or by the reflected power method. In any case, the "optimizing as you go" method should be the rule, because the plasma acts as a dielectric, and the cavity resonances will be broadened and shifted. This also means frequent re-tuning of the sources will be necessary.

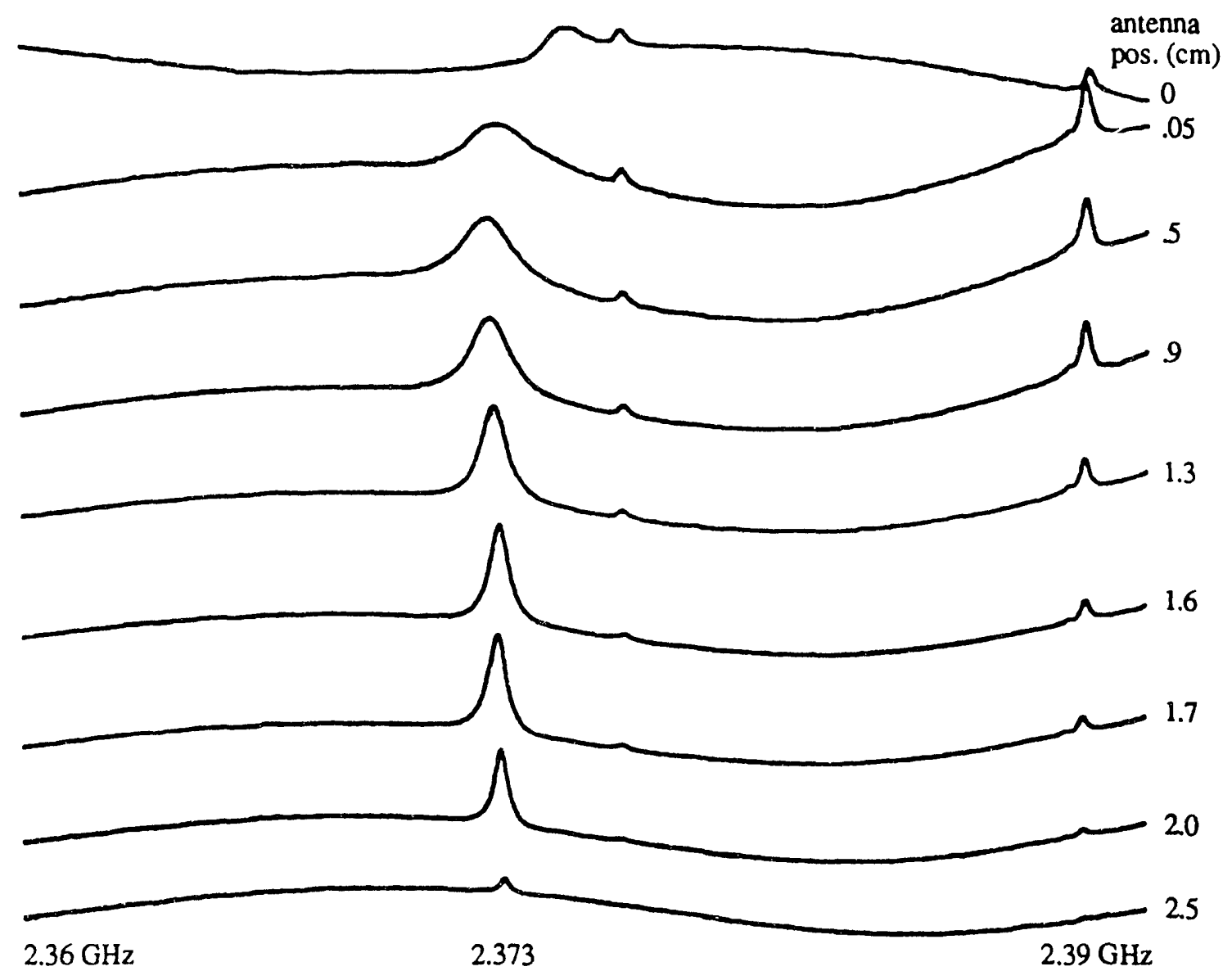

Fig. 3.15 Reflected power of the $\mathrm{TM}_{1,1,20}$ resonance. The baseline is full reflected power. The peaks are minimum in reflected power. The antenna position is the distance withdrawn from complete insertion. The frequency shift and decrease in $\mathrm{Q}$ is obvious as the antenna protrudes more into the cavity. The $Q$ ranges from 7200 to 1400 . At full insertion the antenna is touching the metal end cap of the ceramic tube. This causes a big change in the coupling. A distance of $1.6 \mathrm{~cm}$ is best in terms of minimum reflected power. 


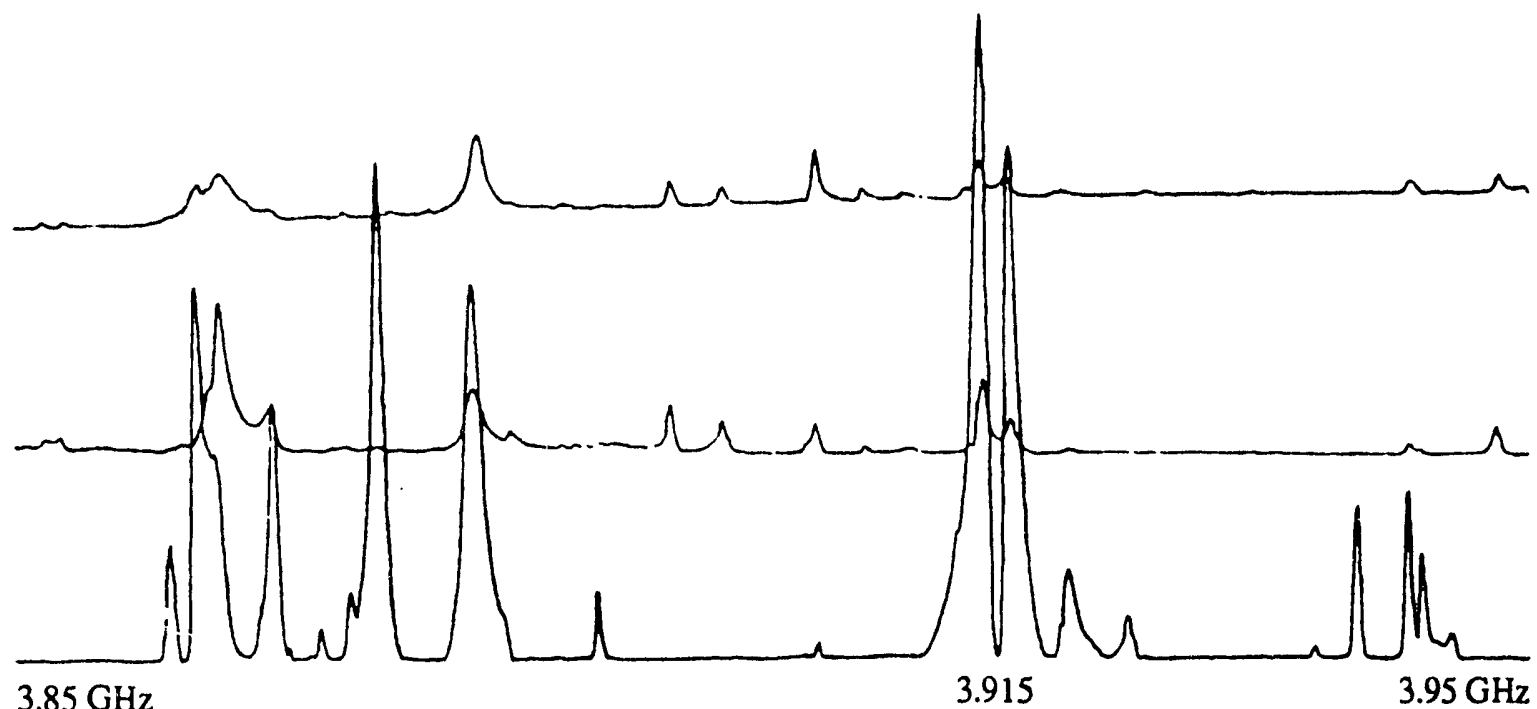

Fig. 3.16 A resonance plot of the cavity in the vicinity of the second harmonic heating frequency. Input is at 9 o'clock. The bottom trace is the signal at 3 . The top two are the signal at 6 and 12. Mode identification is confused by peaks that appear at 6 and 12 but not at 3 . The group at 3.915 $\mathrm{GHz}$ appears to be the best choice.

\subsection{Plasma Decay and Timing Control}

\subsubsection{Stopping ECRH and Gas Flow}

A hot electron plasma can be created in a steady state by use of the microwaves. However, the nexi st, $p$ is to stop the microwaves and gas feed to allow the cold electron component to escape. To this end electrically controlled switches are installed in the gas flow line and in the coax cables that carry the microwaves to the vacuum chamber.

The solenoid valve was mentioned previously. It is placed in the $1 / 4$ in. stainless steel tube from the gas feed tank to the plenum chamber. The solenoid is energized by 115 volts $\mathrm{AC}$, which holds open a valve. When the power is turned off, a spring drives the valve shut. It responds in a few milliseconds. 
The RF relay switch contains a solenoid that is activated by 28 volts DC. The solenoid drives contacts that direct the input between one of two outputs. One output is connected to the cable/antenna and the other output goes to a dummy load. The switches can handle 200 watts of power. The relays have been observed to switch in $5 \mathrm{~ms}$.

\subsubsection{Delay Box}

The delay box controls the electrical signals that operate the switches. A variable delay is possible between the stopping of the gas flow and the cutoff of the ECRH. This was done based on the theory that it is a good idea to give the background gas pumping a head start before the plasma decay begins.

Extra background gas would increase the plasma decay through increased collisions. Pumping the gas first should lengthen the decay when it begins. Pumping for too long will reduce the source of the plasma. The ionization rate will decrease and the plasma density will drop. Then the plasma will decay to zero sooner. Discovering the optimum delay will be a trial and error process.

The delay box has gone through several revisions. Fig. 3.17 gives a schematic view of how this control system operates. Initially the signal delay was a separate pulse generator with delay capability. Later, after installation of the pulser coil, the signal delay was accomplished with the use of 555 IC timer chips. The delay knob became a variable potentiometer used in an RC circuit in conjunction with the 555. Two separate signal delays were also added for the low frequency (LF) and the high frequency (HF) microwaves. This was done with the idea of continuing the HF power after the LF power was stopped. The thought was that the HF power would selectively maintain the hot electron population without having the LF heating driving up the low energy end of the distribution. Finally another signal delay and relay were added to operate the switch that initiates the pulsed magnetic field. Its variable delay would allow the cinoice of when to begin the pulsed field during the decay process. 


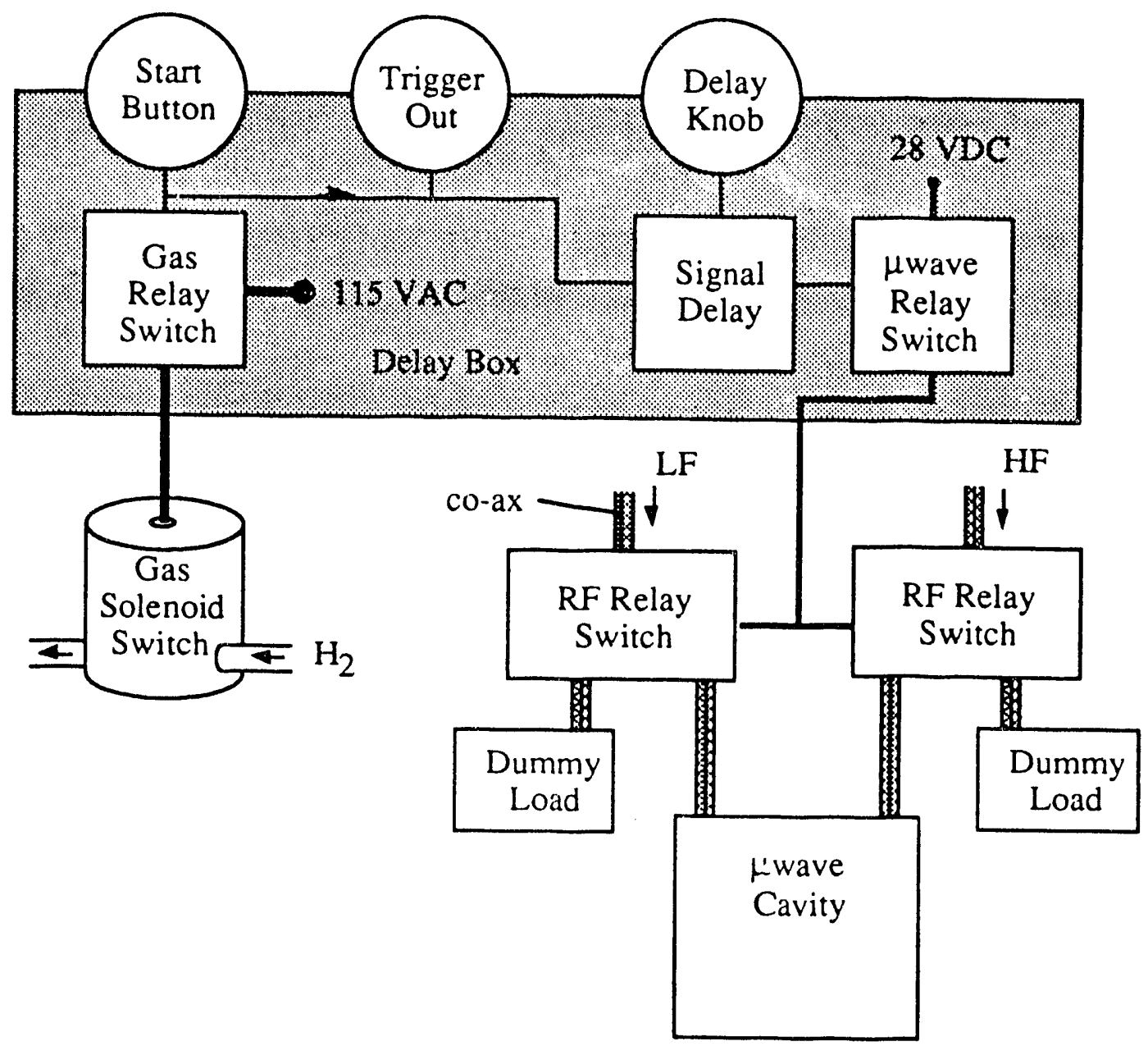

Fig. 3.17 A schematic diagram for the delay box. The trigger is used to time diagnostics. When the solenoid is energized, gas flow is stopped. When the RF relays are energized, the microwaves a:e directed into the dummy load. 


\section{Chapter IV The Hot Electron Plasma}

An evaluation is presented in this chapter of the capability of the Mirrortron machine to produce a high electron temperature plasma. This includes a study of the plasma decay. This discussion begins with a description of the two main diagnostics used to measure the plasma electron temperature and plasma density.

The $\mathrm{x}$-ray detection system measures the $\mathrm{x}$-ray emission from the plasma confinement vessel in two ways. One way is as a spectrometer. From the energy spectrum of the emitted photons, the electron temperature can be inferred. This is a simple and straightforward way to tell if we are successful in heating the plasma electrons to high temperatures.

The other way is to use the $\mathrm{x}$-ray detector as a total photon counter. This is a basic way of determining the relative intensity of $\mathrm{x}$-ray emission. An increase in intensity indicates an increase in the hot electron density or an increase in the electron temperature or both. In either case, such an indication means a move towards more desirable plasma conditions.

Apart from $x$-ray intensity in steady state, photon counting can be used during the decay of the plasma. The rate of decay of $x$-ray intensity will show how long the hot 
electron component remains trapped, which is more important than knowing the characteristic decay time for the plasma as a whole.

To estimate the density, the difference is measured between the vacuum resonant frequency and the resonant frequency in the presence of the plasma. The resonant frequencies depend on the dielectric constant of the plasma medium, which in turn depend on the plasma density. For this experiment, the frequency shift has a linear dependence on the plasma density. A steady-state density or a point on a decay curve (density vs. time) can be measured.

\subsection{Electron Temperature}

\subsubsection{X-ray Detection System}

The $\mathrm{x}$-ray detection system consists of a NaI scintillation crystal connected to a photomultiplier tube (PMT). The crystal and the PMT are combined in one package. The crystal dimensions are $4 \mathrm{~cm}$ long and $5 \mathrm{~cm}$ in diameter. The crystal detector works together with a Canberra multichannel analyzer (MCA). The MCA provides high voltage to the PM tube dynode chain, as well as power for a pre-amplifier mounted near the detector. Signal pulses from the detector are sent to the pre-amplifier, then to the MCA where they are processed. Energy distributions or counting statistics can be done.

Since the tube will be placed close to the vacuum vessel, it will be located in a high magnetic field. This will adversely affect the operation of the dynode multiplier. For this reason an attempt to shield the detector was made. The shield is a $39 \mathrm{~cm}$ long tube consisting of three layers. There is a $0.32 \mathrm{~cm}$ thick outer copper shield, a $0.64 \mathrm{~cm}$ thick soft iron layer, and a $0.08 \mathrm{~cm}$ thick inner cylinder of $\mu$ metal. This configuration is shown in fig. 4.1. Over the course of the experiment it was determined that the shielding is better when the detector and shield are oriented perpendicular to the field, as one might expect, even when placed between the main mirror field coils. However, the strongest $x$-ray emission is directed along the axis. 

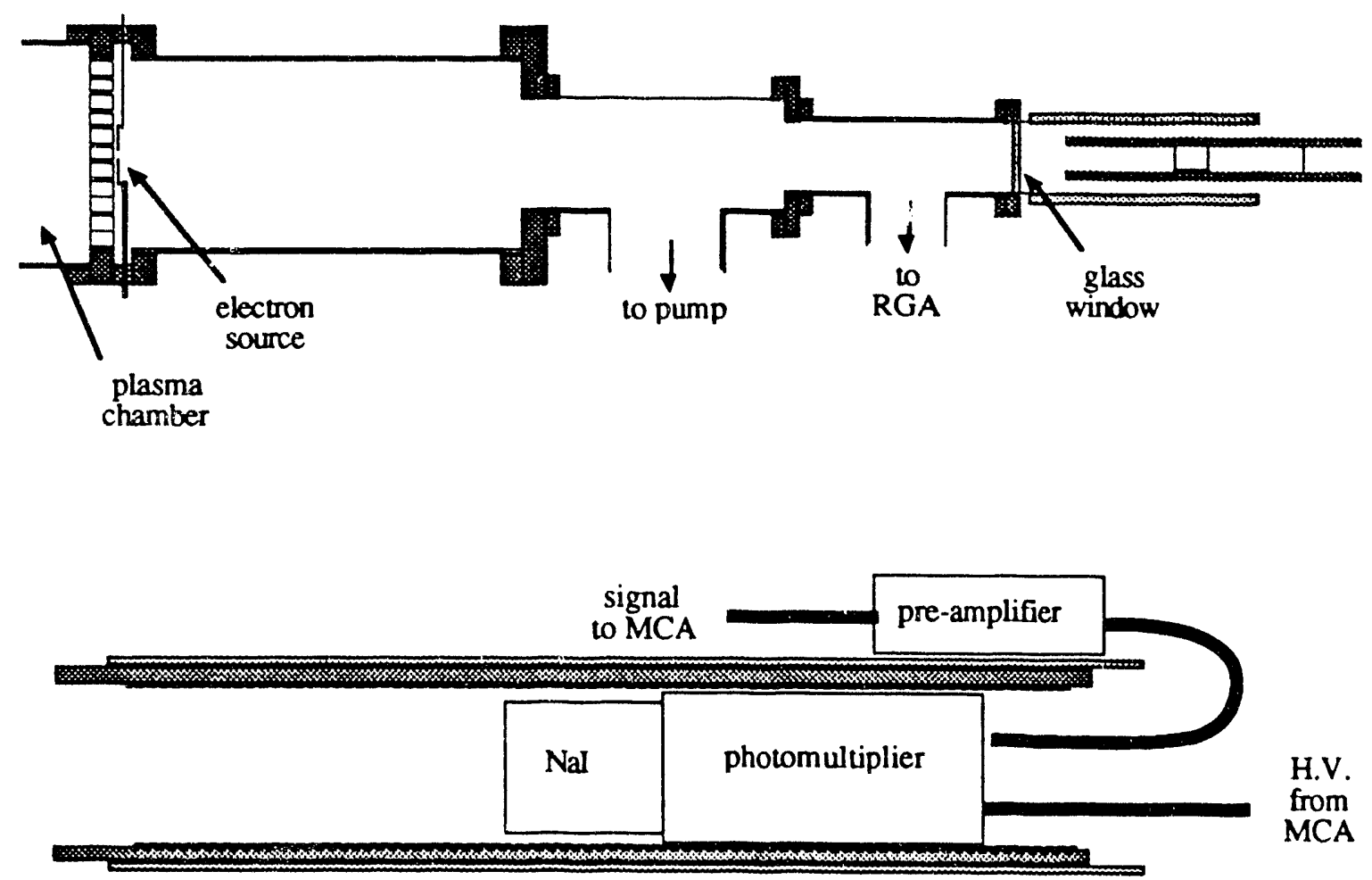

Fig. 4.1 The Nal crystal detector and its final position with respect to the vacuum vessel. Here the detector is $215 \mathrm{~cm}$ away from the center of the plasma. Also shown is the magnetic shielding protecting the photomultiplier tube.

\subsubsection{X-ray Emission}

\subsubsection{Free Electron-Ion Bremsstrahlung}

The purpose of the $\mathrm{x}$-ray detector is to determine the hot electron temperature. This was to be done by examining the bremsstrahlung radiation from free electron-ion collisions. To understand how the measurement is done, first note that the power emitted from a unit volume per unit energy interval is[13]

$$
\frac{d P}{d \varepsilon}=K \sqrt{\frac{\chi_{H}}{k T_{e}}} e^{-\varepsilon / k T_{e}}
$$


$\mathrm{K}$ is a constant independent of the electron temperature and $\mathcal{E}=\mathrm{hv} . \chi_{\mathrm{H}}$ is the hydrogen ionization potential. This suggests that the temperature can be discovered by doing an energy spectrum analysis of the emitted x-rays. We need only examine the slope when plotted on a log scale.

When implementing this procedure in the experiment, it was necessary to restrict the line of sight of the detector to prevent the electron-wall radiation from confusing the spectrum. This radiation was expected to be at least an order of magnitude more intense. The free electron bremsstrahlung radiation was collimated by a small hole in a lead block placed behind the collimation tubes. This allowed a view of an area just smaller than the center hole in the ventilation flange on the far side of the vacuum chambe::

The $\mathrm{x}$-ray detector was first placed on-axis next to the gas feed section, detecting $\mathrm{x}$ rays that passed through a thin aluminum flange. There was not a good signal from the detector because the collimation was too severe, and the magnetic shielding was not sufficient to keep the PM tube unaffected.

Geiger counters were used to detect the first x-rays emanating from the sides of the vacuum vessel. This came from the energetic electrons striking the wall. When the scintillation detector was used to examine these $\mathrm{x}$-rays, a near linear, high energy tail was seen, when plotted on a log scale. These $\mathrm{x}$-rays were a few hundred $\mathrm{keV}$ in energy.

\subsubsection{Electron-Wall Bremsstrahlung}

At this point I decided to use the electron-wall bremsstrahlung to analyze the electron temperature. Kulenkampff [14] gives an empirical formula for the intensity of $\mathrm{x}$-radiation at a certain frequency hv from a monoenergetic electron beam of energy $\varepsilon$ as a function of beam current.

$$
\begin{aligned}
\mathrm{I}(\mathrm{hv}) & =\mathrm{B}(\mathcal{E}-\mathrm{hv}) \mathrm{i} & & \mathrm{hv}<\mathcal{E} \\
& =0 & & h v>0
\end{aligned}
$$


$B$ is an empirical constant and $i$ is the beam current. To find the radiation from a plasma with a spectrum of energies, it it necessary to substitute an equation for the current, where the current is a function of electron energy.

$$
\begin{aligned}
\mathrm{i}(\mathcal{E}) & =\mathrm{j}(\boldsymbol{\varepsilon}) \mathrm{A} \\
& =\mathrm{n}(\boldsymbol{\mathcal { E }}) \mathrm{qv} \mathrm{A}
\end{aligned}
$$

$A$ is a unit area of the vacuum vessel struck by an element of the plasma that has energy $\mathcal{E}$ and with density $n(\mathcal{E})$. The velocity of these particles is $v=\sqrt{2 \varepsilon / m}$ in the non-relativistic limit. The density $n(\mathcal{E})$ is just the electron distribution function. A suitable loss-cone distribution model (after integrating over angles) that will be used in the calculation is

$$
\mathrm{n}(\boldsymbol{E})=\alpha \mathrm{n}_{0} \varepsilon^{-\mathcal{E}}
$$

Here, the electron energy is made dimensionless by normalizing with the electron temperature $\mathrm{kT}$, i.e., $\varepsilon=\mathrm{mv}^{2} / 2 \mathrm{kT}$. $\alpha$ is a normalization constant. Hence, by combining eq. 4.2-4.4, the intensity of radiation from a unit area is proportional to

$$
\begin{aligned}
\mathrm{I}(\mathrm{hv} v) & \propto \int_{\mathrm{h} v / \mathrm{kT}}^{\infty}(\varepsilon-h v / \mathrm{kT}) \varepsilon^{3 / 2} \mathrm{e}^{-\varepsilon} \mathrm{d} \varepsilon \\
& \equiv \mathrm{I}_{\mathrm{o}}\left\{\left(\frac{\mathrm{h} v}{\mathrm{kT}}\right)^{5 / 2} \mathrm{e}^{-\mathrm{h} v / \mathrm{k} T}+\frac{3}{2} \Gamma\left(\frac{5}{2}, \frac{\mathrm{h} v}{\mathrm{kT}}\right)\right\}
\end{aligned}
$$

where $\mathrm{I}_{\mathrm{o}}$ is a proportionality constant. We are interested in how the intensity will be displayed on a log scale on an MCA, especially in the high photon energy range. The expression for $\mathrm{I}(\mathrm{h} v)$ can be simplified for the case $\mathrm{h} v$ » $\mathrm{kT}$. An asymptotic expansion can be made for the incomplete gamma function in eq. 4.5. In addition, when the log of $I(h v)$ is 
taken, further expansions can be made based on hv $" k T$. Let $u=h v / k T$. Eq. 4.5 is expanded and the natural $\log$ is taken.

$$
\begin{aligned}
I(u) & =I_{0}\left\{u^{5 / 2} e^{-u}+\frac{3}{2} u^{3 / 2} e^{-u}\left(1-\frac{3}{2 u}\right)\right\} \\
\ln (I(u)) & =-u+\frac{5}{2} \ln (u)+\left(\frac{3}{2 u}-\frac{9}{4 u^{2}}\right)+\ln \left(I_{0}\right)
\end{aligned}
$$

In the energetic tail of the x-ray distribution the linear behavior with hv (as displayed on a log scale) should be dominant. By determining the slope in the high energy, linear portion of the spectrum, a reasonable estimate of the electron temperature can be made.

\subsection{Plasma Density}

\subsubsection{Frequency Shift Measurement}

The method of density measurement used in this experiment depends on measuring the frequency shifts of cavity resonances. In eq. 3.15 it is seen how the frequency depends on the index of refraction of the filling medium. The index $\tilde{n}$ is less than one for a plasma and hence the frequency will be shifted up.

The set up to measure the frequency shift is shown in fig. 4.2. The procedure is as follows. A separate microwave oscillator with a co-ax cable/antenna placed in one of the ports is used to excite one of the fundamental TM resonances, $m=0, n=1$, and $p=0,1$ or 2 . These resonances are chosen so that they can be distinguished from the higher ECRH frequencies and to allow a separation band in which a low pass filter can let the probing microwaves through and block the heating power from returning to the oscillator. The first TM vacuum resonance, $f_{0,1,0}=860 \mathrm{MHz}$, is not easily excited. In the presence of the plasma, the peak is broadened and becomes virtually impossible to detect. The second TM vacuum resonance was typically used; $\mathrm{f}_{0,1,1}=866 \mathrm{MHz}$. These resonances would vary by about $1 \mathrm{MHz}$ because of temperature variations of the vessel dimensions due to bake out. 


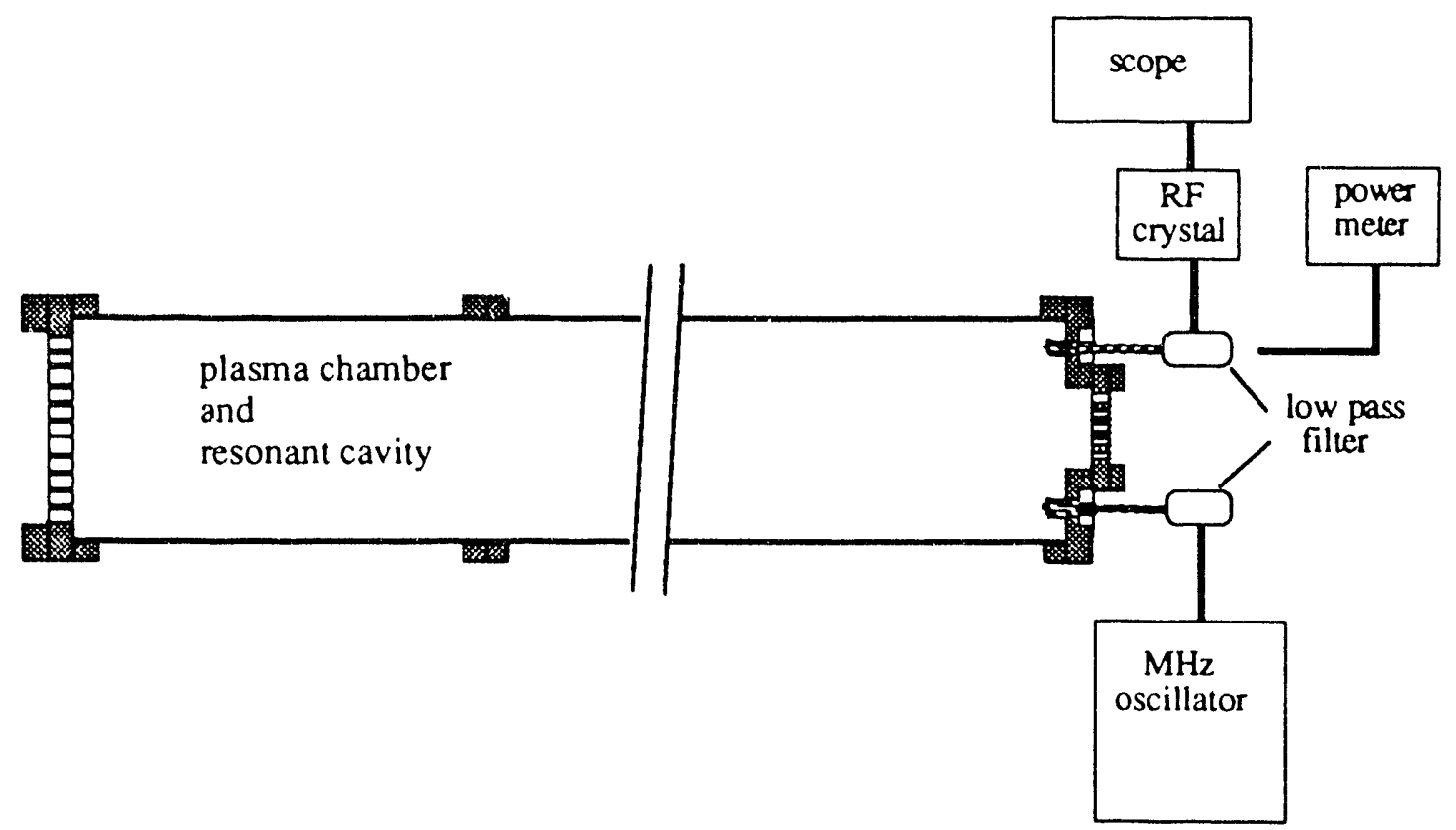

Fig. 4.2 This is the set-up to measure the frequency shift of cavity resonances caused by the plasma. The oscillator is tunable and is used to search for the shifted resonance.

A second antenna is in the last port and connects to a power meter or a crystal which puts out a voltage proportional to the electric field traveling in the cable. Either path may be used to detect the excited mode. A filter is placed in-line on this antenna so that the high power ECRH frequencies do not swamp the detector. The oscillator is then tuned until the second antenna detects a peak in the cavity energy density. The oscillator frequency is then equal to the resonant frequency.

The density decay can be estimated if the oscillator is set at or near the vacuum resonance. The RF crystal is used because of its fast response, and it can be connected to an oscilloscope. Once the RF is shutoff then the decay time is roughly the time it takes for the oscillator energy within the cavity to peak. This is not a plot of density versus time, however. 
The time decay of the plasma density can be measured but is a little more difficult because only a single data point can be taken per decay. The plasma must be reformed after each shot with conditions nearly identical to the previous plasma. In this method the oscillator is preset to a certain frequency corresponding to a certain density. The cavity energy density is then monitored as the plasma decays. The signal will pass through a peak and the time to the peak is the time to reach that density. For each shot the oscillator frequency is changed. Over several shots the density decay behavior can be determined

There are some problems to be concerned with. For one thing, the frequency shifts can be larger than the difference between frequencies of different modes. So one must be careful of which mode is being measured. The best way to proceed is to start with the oscillator tuned to the vacuum resonance. The first measurement will be the time to decay to vacuum. Then by tuning the oscillator up in small increments, the shifted resonance peak can be followed up in frequency.

Another problem might be fluctuating density. Several peaks in the power level vs. time trace might not mean several resonances shifting past the oscillator frequency, but rather, one resonance shifting back and forth across the oscillator frequency. Detecting the particle end-loses (see sect. 4.5.1) should help identify fluctuations in density.

\subsubsection{Derivation of Shift Formula}

Buchsbaum et. al. [15] describes a perturbation method in which the frequency shift can be calculated. Here, this method is applied to a cylindrical plasma column in which a magnetic field is oriented along the length of the plasma. The limits of validity for this method are determined by the requirement that the plasma skin depth is greater than the plasma radius. This simply states that if the plasma is dense enough to shield out the cavity fields from its interior, then no information can be gathered about its interior. This requirement is stated as 


$$
\frac{\omega_{p}^{2}}{\omega^{2}}<1+\left(\frac{c}{\omega a}\right)^{2}
$$

where $a$ is the plasma radius. If $a=10 \mathrm{~cm}$ and $f=860 \mathrm{MHz}$ then we must have $\mathrm{n}_{\mathrm{c}}<1.2$ $10^{10} \mathrm{~cm}^{-3}$. The expression for the shift $\Delta \omega$ is given by

$$
\frac{\Delta \omega}{\omega}=\frac{i \int E_{0} \cdot\left(\sigma \cdot \mathbf{E}_{0}\right) d V}{2 \omega \varepsilon_{0}} \frac{\int E_{0}^{2} d V}{d V}
$$

where $\mathbf{E}_{\mathbf{0}}$ is the vacuum cavity field and $\sigma$ is the conductivity tensor. The integration is over the volume of the cavity. Using the conductivity tensor for a magnetized plasma with the magnetic field along the $z$ direction, and using the TM modes where $m=0, n=1\left(E_{\theta}=\right.$ ()) the equation above reduces to

$$
\frac{\Delta \omega}{\omega}=\frac{i}{2 \omega \varepsilon_{0}} \frac{\int \sigma_{r r} E_{r}^{2}+\sigma_{z z} E_{z}^{2} d V}{\int E_{o}^{2} d V}
$$

where the cold plasma conductivity tensor elements are $\sigma_{Z Z}=\frac{-i \varepsilon_{Q} \omega_{D}{ }^{2}}{\omega}$ and $\sigma_{r r}=\frac{-i \varepsilon_{0} \omega_{p}^{2} \omega}{\omega_{c}^{2}-\omega^{2}}$. Collisions can be important but are neglected here because the density is low enough that the collision frequency is very small compared to the resonant frequencies.

The radial portion of the interaction integral (eq. 4.9) will be neglected in the subsequent derivation. This is easily done for the fundamental $\mathrm{p}=0$ mode because $\mathrm{E}_{\mathrm{r}}=0$. To justify this for the $\mathrm{p}=1,2$ modes, consider the ratio $\mathrm{E}_{\mathrm{r}} / \mathrm{E}_{\mathrm{z}}$. Their relative scales (see eq. 3.16) are determined by the factor $p \pi R / d \chi_{01}=.11 \mathrm{p}$. In addition, the transverse conductivity will be constrained by the magnetic field. Therefore the ratio of conductivities, $\begin{aligned} & \sigma_{\pi}=\omega^{2} \\ & \sigma_{x z}\end{aligned} \omega_{c^{2}}^{2}-\omega^{2}$, is approximately 0.2 . Another argument is that the radial field is weakest 
in the near axis region where the plasma is concentrated. Hence, the first term in eq. 4.9 will contribute very little.

To solve eq. 4.9 for the $p=0$ mode, a uniform density column of radius a is assumed. Eq. 4.9 becomes

$$
\begin{aligned}
\frac{\Delta \omega}{\omega} & =\frac{\mathrm{i}}{2 \omega \varepsilon_{0}} \frac{\mathrm{a}^{2}}{\mathrm{R}^{2}} \frac{\sigma_{\mathrm{zz}}\left(\mathrm{J}_{0}^{2}(\xi)+\mathrm{J}_{1}^{2}(\xi)\right)}{\mathrm{J}_{1}^{2}\left(\chi_{01}\right)} \\
& =\frac{\omega_{p}^{2}}{2 \omega^{2}} \frac{\mathrm{a}^{2}}{\mathrm{R}^{2}} \frac{\mathrm{J}_{0}^{2}(\xi)+\mathrm{J}_{1}^{2}(\xi)}{\mathrm{J}_{1}^{2}\left(\chi_{01}\right)}
\end{aligned}
$$

The Bessel function argument is $\xi=\chi_{01}$ a/R. The uniform plasma density, $n_{0}$, is contained in $\omega_{\mathrm{p}}{ }^{2}$. If $10 \mathrm{~cm}$ is used for the plasma radius then eq. 4.10 reduces to,

$$
\mathrm{n}_{\mathrm{o}}=2.310^{7} \Delta \mathrm{f}
$$

The density has units of $\mathrm{cm}^{-3}$ and $\Delta \mathrm{f}$ is in $\mathrm{MHz}$. The resonant frequency for the $\mathrm{p}=0$ mode was used for $f=\omega / 2 \pi=860 \mathrm{MHz}$.

In cases where the plasma is hot enough that its temperature can not be ignored, the conductivity tensor must be modified. Drummond [16] gives $\sigma$ for a hot plasma. The hot plasma theory allows the cold tensor element, $\sigma_{\mathrm{zz}}$, to be modified by including a normalized function that depends on the electron temperature and the relevant frequencies, i.e., $\sigma_{z z} \rightarrow \sigma_{z z} F\left(\omega, \omega_{p}, k T\right)$

$$
F\left(\omega, \omega_{p}, k T\right)=\frac{1+\frac{\langle v\rangle_{\text {th }}^{2}}{c^{2}}\left(\frac{\omega^{2}}{\omega^{2}-\omega_{c}^{2}}\right)}{1+2 \frac{\omega_{p}^{2}}{\omega_{c}^{2}} \frac{\langle v\rangle_{\text {th }}^{2}}{c^{2}}}
$$


The squared average thermal velocity is $\langle v\rangle_{\text {th }}^{2}=k T / m$. In the case that $\omega<\omega_{c}$, the plasma conductivity is reduced. This reduces the interaction between the plasma and the probing fields. For the range of frequencies an ${ }^{2}$. densities in the Mirrortron, $F$ is about 0.92 to 0.96 . In the case of relativistic temperatures, the thermal velocities will not be as great as determined in eq. 4.12. However, the effect on F is small, and it tends to move the value of F closer to one. Hence, a high electron temperature will result in only a few perceni difference in the frequency shift coefficient.

When the $p=1,2$ modes are used, there is a $3 \%$ increase in the frequency shift coefficient if the same uniform plasma column is assumed. When different spatial distributions are assumed, there are bigger increases. For example, if an axial density profile like the ones shown in fig. 3.10 are used, then increases of 10 to $90 \%$ are obtained. The biggest increase comes from the $T_{\perp}=T_{\|}$distribution and the $p=1$ mode. This mode has an axial electric field node at the midplane, resulting in a smaller plasma-field interaction. If a parabolic radial density profile is assumed, $n_{e}=n_{0}\left(1-r^{2} / a^{2}\right)$, then there is a factor of two increase in the shift coefficient. However, the Mirrortron plasma will have a more flat distribution than this. There will be increases if the plasma has a smaller radius, for example, approximately $25 \%$ if the radius $\mathrm{a}=7.5 \mathrm{~cm}$ instead of $10 \mathrm{~cm}$.

Clearly the most important effect in this theory is the plasma distribution. Details of the exact electric field in the cavity or the plasma temperature are unimportant. A smaller plasma means a smaller interaction with the field. This means that a greater plasma density is necessary to cause the same frequency shift. Considering the actual spatial distribution of the plasma, there is approximately a factor of two increase over the coefficient in eq. 4.11 . In light of this, an order of magnitude formula will be used:

$$
\mathrm{n}_{\mathrm{o}}=510^{7} \Delta \mathrm{f}
$$




\subsection{Hot Plasina Development}

\subsubsection{Magnetron Performance}

The first thing that was done was to check the performance of the magnetrons and test their ionizing capability by trying to break down the gas and produce a plasma. The low frequency (LF) magnetron was operated at $2373 \mathrm{MHz}$. This worked well in terms of low reflected power. Breakdown was achieved easily with this frequency. The high frequency (HF) magnetron was operated at $3912 \mathrm{MHz}$. A limited frequency scan around the HF value showed that it was best in terms of low reflected power. Both sources were adjusted to send 30 to 50 watts of power into the cavity.

One noticable problem was that the magnetrons tended to drift in frequency. The magnetron tubes took a long time to warm up, and they had to come to a new equilibrium every time the cathode voltage was changed. The frequency and bandwidth of the output were also a function of the cathode voltage. In addition, when the HF power was increased, a fraction of this power was coupled back to the LF magnetron and changed its operating mode. In general, frequent re-tuning was necessary.

\subsubsection{First X-rays}

Argon was first used as a supply gas so that a heavy ion plasma could be created. A visible discharge was achieved with argon, however, no x-rays were detected. Geiger counters were used in addition to the $\mathrm{NaI}$ detector. The suspected problem was radiation damping, i.e., the microwave field excited several atomic levels of argon and other complex atoms, and these atoms re-radiated the energy.

For this reason we switched to hydrogen. Hydrogen is a simple atom that ionizes easily. The first $x$-rays detected were the result of electron-wall collisions. The photons were energetic but low intensity. There was no visible discharge. The $\mathrm{NaI}$ detector analysis of this radiation produced a spectrum similar to fig. 4.3. The maximum photon energies were 200 to $300 \mathrm{keV}$, and the electron temperature was 30 to $45 \mathrm{keV}$. 
The background gas pressure was $110^{-7}$ torr and the $x$-ray level began to increase when $210^{-7}$ torr of $\mathrm{H}_{2}$ was supplied to the vacuum. However, the density was negligible, causing barely discernible frequency shifts. As the $\mathrm{H}_{2}$ pressure increased above $410^{-7}$ torr, a visible discharge would begin to form. In this stage the frequency shift was 10 to 20 $\mathrm{MHz}\left(0.5\right.$ to $\left.110^{9} \mathrm{~cm}^{-3}\right)$. The plasma density increased with the gas pressure until $1.210^{-6}$ torr then the density was constant. Here it was noticed that the density could be increased when the LF frequency was increased by a few $\mathrm{M}$ Hz. The plasma was shifting the resonant heating frequen:y as well. The LF frequency was increased $4 \mathrm{MHz}$ to $2377 \mathrm{MHz}$.

There were only minimal $x$-rays during the glow discharge, however. It was thought that the discharge knocked impurity gasses off the walls which increased radiation damping. Raising the gas pressure to $1.810^{-6}$ tor and above as a means of discharge cleaning improved plasma radiation levels when the gas pressure was again lowered. Also, experimentation with the magnetic field indicated that a midplane field of 420 to 480 gauss $\left(R_{m}=2.2\right.$ to 2.5$)$ increased the radiation levels as well. This is to be expected, because of improved confinement for $h:$ oh perpendicular energy electrons.

At this time heat tapes were added to bake out the walls, and an electron source was added to make breakdown easier and to increase plasma density without increasing the background gas pressure. Also a residual gas analyzer (RGA) was added to detect impurity gasses.

\subsubsection{First Plasma with X-rays}

The RGA showed that the discharge produced many impurities, especially water. Several hours of this type of discharge cleaning, as well as baking out the vessel, reduced the impurities to an acceptable level. Clean hydrogen gas made it possible to create $\mathrm{x}$-rays during a glow discharge. Purer gas meant that the initial pressure required to start a discharge was increased to $1.610^{-6}$ torr. The discharge could be maintained at lower pressures, but it was unstable and could easily disappear. 
Eventually the hot plasmas were reproduced regularly with 50 watts while increasing the heating frequency to account for plasma shifts. Frequencies of 2377 and $3916 \mathrm{MHz}$ were typical. The $\mathrm{Nal}$ detector measured an $\mathrm{x}$-ray flux of $8 \mathrm{~K}$ counts/sec from the side of the vacuum vessel. About this time it was moved to its position shown in fig. 4.1. Even though the detector was further away from the plasma, the $\mathrm{Nal}$ count rate went up to $70 \mathrm{~K}$ $\mathrm{cts} / \mathrm{sec}$, an increase by a factor of 9 . Clearly more photons were directed along the axis. Temperatures measured from this position reached $90 \mathrm{keV}$.

The highest density plasma was achieved with $\mathrm{H}_{2}$ gas pressures of up to $310^{-6}$ torr. Frequency shifts for this density were up to $40 \mathrm{MHz}$, meaning a density of $210^{9} \mathrm{~cm}^{-3}$. The $x$-ray flux damped out at higher gas pressures. The greatest $x$-ray flux was emitted from plasmas with minimum $\mathrm{H}_{2}$ gas flow. Such plasmas were not practical because they were unstable and disappeared with any variation in the microwave source frequency.

Once the plasma reached the high density, high temperature stage, niether the $x$-ray flux nor the density changed noticeably when the frequency of the HF microwaves changed by a few $\mathrm{MHz}$. This was because the wide HF bandwidth and the many plasma-broadened resonance frequencies allowed a continuous coupling between modes. In addition, the plasma was not much affected by the exact value of the magnetic central field. Since the plasma temperature and density were high enough now, it seemed worthwhile to sacrifice a little on the high temperature by increasing the central magnetic field to reduce the mirror ratio. The maximum current output of the central magnet power supply resulted in a midplane field of about 620 gauss and a mirror ratio of 1.7 .

At this time, a more extensive search of the frequency range of the HF magnetron led to a discovery of a more efficient frequency with which to heat the plasma. A frequency of $3644 \mathrm{MHz}$ was used, and plasma temperatures of over $100 \mathrm{keV}$ could now be measured. The HF frequency corresponds to a resonance at 650 gauss, so the resonance was near the midplane. 
At this stage of the experiment, the electron temperature reached $300 \mathrm{keV}$ on two occasions. This was accomplished using a relatively low gas pressure of $1.010^{-6}$ torr. Also higher power was used, about 80 watts. This power level could not be maintained for very long because the cables and antennae were not designed to dissipate this much power. In general, plasmas with temperatures of 80 to $100 \mathrm{keV}$ were typical because they were more stable, and were maintained with moderate power.

Fig. 4.3 is a typical $\mathrm{x}$-ray spectrum taken with the $\mathrm{NaI}$ detector. The high energy tail from about $600 \mathrm{keV}$ up is linear on a log scale. The temperature calculated from the slope in this energy range is $80 \mathrm{keV}$. The $\mathrm{H}_{2}$ gas pressure in this case is about $110^{-6}$ torr. The plasma density is about $10^{9} \mathrm{~cm}^{-3}$.

\section{Mirrortron X-ray Emission}

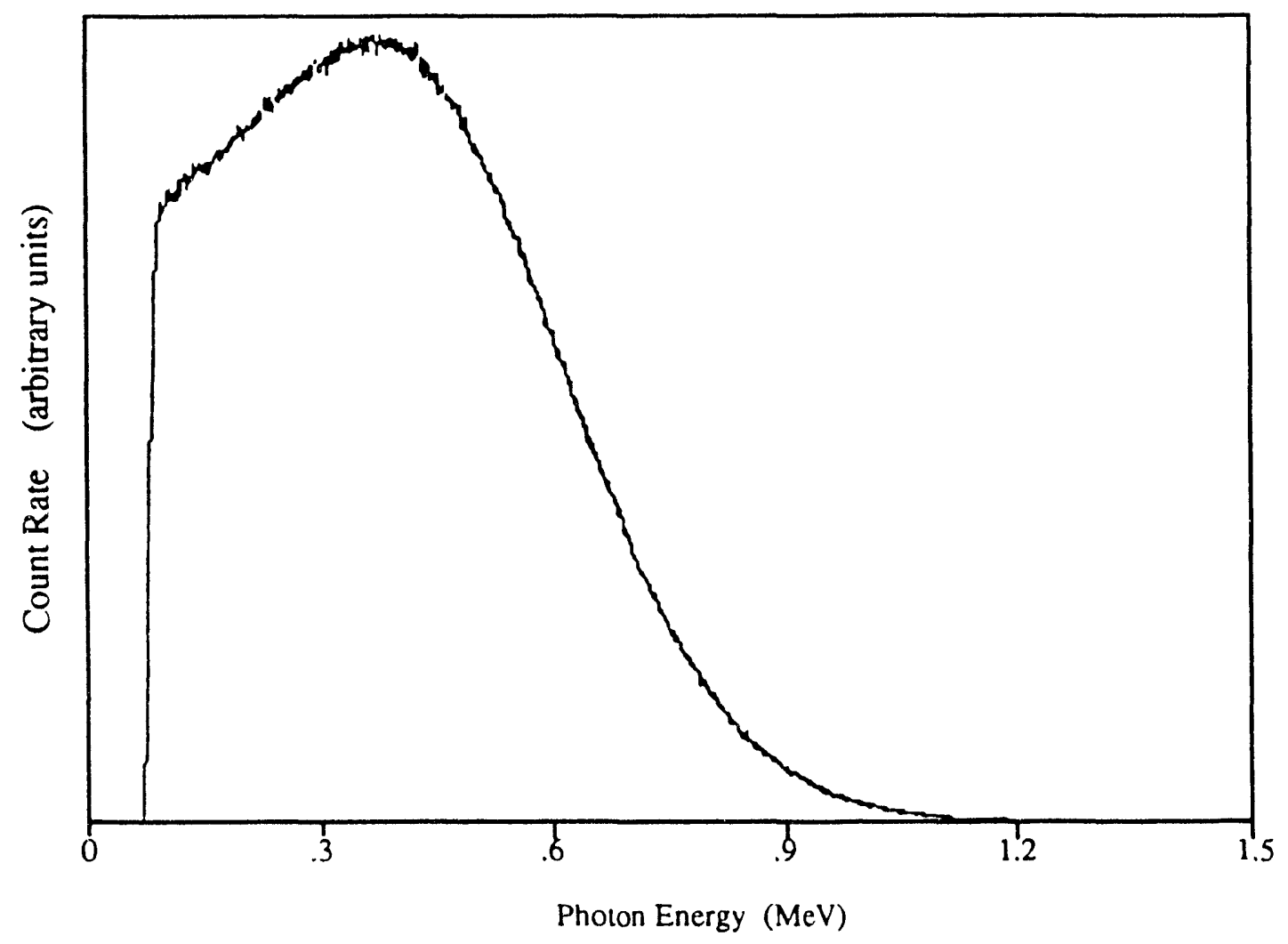

Fig. 4.3 The $x$-ray spectrum measured by the Nal scintillator. The plasma is in steady state. The gas pressure is $1.010^{-6}$ torr. The plasma density is near $10^{9} \mathrm{~cm}^{-3}$. The characteristic temperature is $\mathrm{T}_{\mathrm{e}}=80 \mathrm{keV}$. 


\subsection{Electron Source}

A high temperature plasma can be produced without the help of an electron source.

However, it was tested to see if the temperature or density could be increased further. Fig. 4.4 shows the source in its position just outside the resonant cavity behind the ventilation flange. Electrons with an energy of a few hundred $\mathrm{eV}$ were launched into the cavity.

During discharge operation the $\mathrm{x}$-ray level responded more to the voltage on the anode plate than from electron emission. What response there was to the plate voltage was small and unpredictable. However, it did suggest that there was good conductivity between the plasma and the anode plate, hence, there was good conductivity to the ventilation flange as well. This means that line tying is a good method of stabilization.

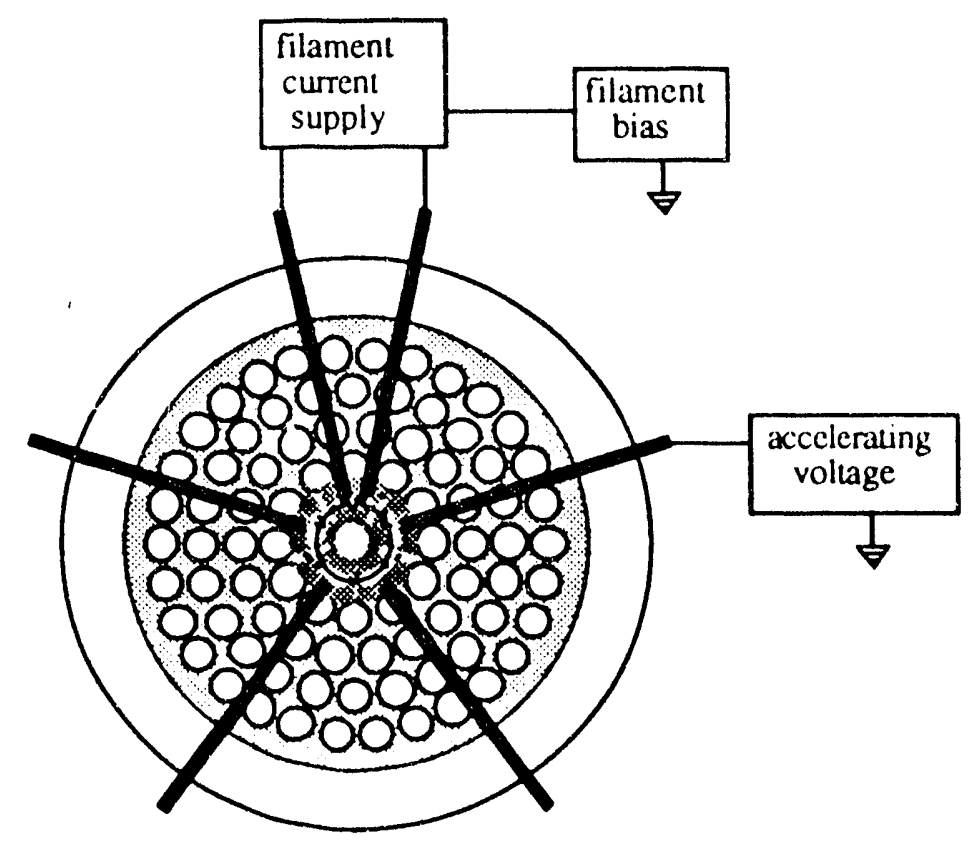

Fig. 4.4 The electron source and its position in front of the ventilation flange. Electrons are accelerated by the positive voltage on the anode plate and are launched into the microwave cavity through the six holes about the center hole of the ventilation flange. The plate could also be used to collect plasma particles. 
By collecting current as a function of the voltage on the plate, data could be taken similar to that of a Langmuir probe. Since the plate is outside the plasma boundary, the curve will not give any information on the plasma potential. All the electrons in the distribution will be shifted in energy by the same amount equal to the plasma potential as they pass through the ventilation flange. Hence, the knee of the I-V curve will correspond to zero bias on the anode plate.

Fig. 4.5 shows two I-V curves. The left curve indicates better confinement because of the smaller escaping electron flux and the higher temperature. With all other measured parameters the same, the plasma would often switch between these modes of confinement. These traces have been corrected for the ion current, which has been subtracted out. Near zero volts the electron current rises exponentially as the voltage on the plate increases to zero and becomes positive. The behavior is $\mathrm{e}^{-\Phi / \mathrm{kT}}$ and from this the temperature of the escaping distribution is found to be $9 \mathrm{eV}$ in both cases. The slope of the log curve at larger negative voltages indicates that there are smaller density components of higher temperatures. Hotter electrons do not follow a Maxwellian distribution in a RF piasma. Therefore, the slope is expected to gently roll over to zero as the plate bias moves to voltages comparable to the electron energies. The ion current for the left trace is $0.19 \mathrm{~mA}$ and $0.89 \mathrm{~mA}$ for the right trace. Electron saturation currents are 2 and $10 \mathrm{~mA}$ respectively.

\subsection{Decay Measurements}

The purpose of this experiment is to provide a hot electron loss-cone distribution, nominally oy allowing the plasma to decay for a shor time to allow the cold electron component to escape. The purpose of the decay measurements is to characterize this behavior. A control box is used to investigate the effect of varying the time between the stopping of the gas flow and the stopping of RF heating. 
Collected Electron Current vs Plate Volta
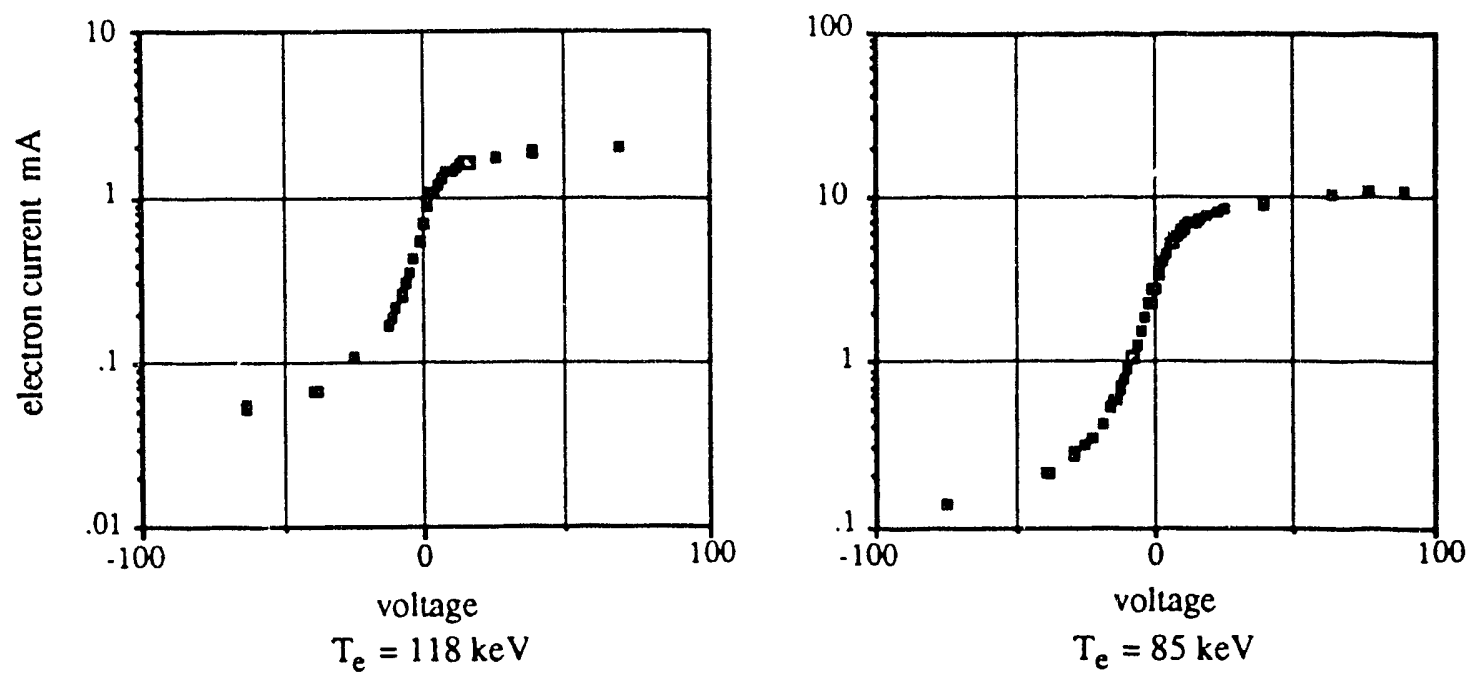

Fig. 4.5 Two current-voitage characteristics using the electron source anode plate. They suggest two different levels of plasma confinement. On the left is a plasma with smaller end losses but a higher electron temperature than the plasma on the right. $\mathrm{H}_{2}$ gas pressure is $910^{-7}$ torr for both cases.

\subsubsection{Measurement Using Electron Source}

The floating potential on the anode plate was used as an indication of the electron and ion end-loss current. Typical voltages were -10 to -15 volts, showing that the electron current was greater. The measurement was done by connecting the anode plate directly to an oscilloscope. The current was discharged through the high input impedance of the scope. These measurements were most effectively used for plasma decay measurements. The decrease in the anode plate voltage described the decrease in escaping plasma particles and thus the decay of the plasma itself. This measurement was only able to characterize the decay of the cold component.

When the gas flow was stopped, the plate voltage would begin to decrease to zero in a time that would last a significant fraction of a second. This indicated the fact that the ECR heating was still producing plasma, but the ionization rate was decreasing. Often during 
this stage there would be oscillations and sudden decreases in the signal level, showing that the plasma was reaching an unstable condition where the ionization rate jumped between high and low levels. Once the RF was shut off there was always a sharp drop in the signal to near zero followed by a decay that never lasted more than a few milliseconds, $20 \mathrm{~ms}$ at the most. The longer the neutral gas decay lasted, the shorter the decay was after RF shutoff. The fast drop corresponds to the fact that the RF heating is not driving the electrons out of confinement and into the loss-cone by diffusion. The $9 \mathrm{eV}$ electrons then escape within microseconds. The subsequent decay is the result of continuing ionization of neutral gas by warm electrons. When the gas pumping lead time is long, the neutral gas pressure will be low and the ionization rate will be small.

\subsubsection{X-ray Intensity Decay}

Once the NaI detector was moved to the on-axis position, the large $\mathrm{x}$-ray fiux now available made it possible to look at the $x$-ray decay. The MCA was set to count the photons every $10 \mathrm{~ms}$ and there were a few hundred counts per time bin. It was expected that this should be a better measure of the hot electron component decay because only the hot electrons could produce $x$-rays.

When the gas was shutoff there was usually a rise in the $x$-ray level. The initial background gas pressure determined how much the intensity increased and how long it stayed at an increased level. The $\mathrm{x}$-ray flux would increase at gas shut-off even for the lowest $\mathrm{H}_{2}$ pressures. As the chance of collision with a neutral decreased then the electrons could be heated to higher energies. This shows that the minimum pressure needed to have a discharge is best in terms of electron temperature. If the gas pressure was initially high then the $\mathrm{x}$-ray level would rise at gas shutoff, but then it would reach a plateau and stay there for a time on the order of a second. As the gas continued to be pumped with the RF on, eventually there would be a drop to zero in intensity that would correspond to characteristic gas pumping times. 
When the RF heating was stopped there was an instant drop in the $x$-ray level (within the 10 ms resolution) then a longer exponential decay. The decrease was by a factor of 10 to 20. Again, the fast drop corresponds to the fact that the RF heating is not driving the electrons out of confinement and to the wall by diffusion. The longer decay represents hot electrons that escape confinement by a collisional process. This decay would last about 200 to $500 \mathrm{~ms}$ typically and appears linear on a log scale. It is good evidence that a population of hot electrons lasts for a significant time beyond cold plasma decay.

\subsubsection{Growth of Oscillator Signal}

The first method of looking at plasma density decay was by exciting the vacuum resonance of the cavity and watching the signal grow as the plasma returned to vacuum.

\section{Gas-RF Delay Time}

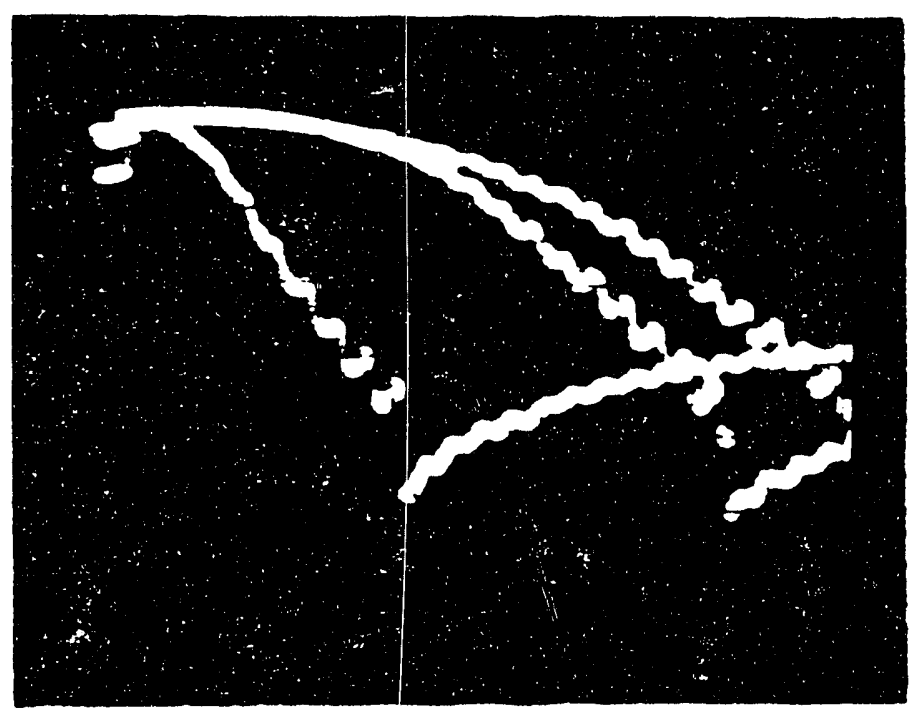

Fig. 4.6 Here are three traces measuring the plasma decay by the growth of resonance method. The RF crystal output is negative. The total scale length is $200 \mathrm{~ms}$. The traces are for a $10 \mathrm{~ms}, 250 \mathrm{~ms}$ and $1 \mathrm{sec}$ delay between the gas shutoff and the RF shutoff. One second results in the fastest decay and $250 \mathrm{~ms}$ is the longest. The initial hydrogen gas pressure was $2.410^{-6}$ torr. 
Using this method, decays of about $200 \mathrm{~ms}$ were seen. Experiments to determine the best choice for the gas-RF delay time were done using this method of density decay. Fig. 4.6 shows some typical signals. Here the oscillator signal was set $1 \mathrm{MHz}$ above the $\mathrm{p}=2$ vacuum resonance. The peaks at the bottom of the traces correspond to a plasma density that shifts the vacuum resonance by exactly $1 \mathrm{MHz}$. The three traces correspond to three gas-RF delay times: $10 \mathrm{~ms}, 250 \mathrm{~ms}$, and $1 \mathrm{sec}$. I looked for the longest decay to vacuum as the best indication of the gas-RF delay time. A quirter second delay produced the longest decay of over $200 \mathrm{~ms}$ for an initial gas pressure of $2.410^{-6}$ torr. If the initial pressure was $2.210^{-6}$ then the longest decay, $110 \mathrm{~ms}$, was for a $10 \mathrm{~ms}$ delay. If the initial pressure was $2.810^{-6}$ then the longest decay, $160 \mathrm{~ms}$, was for a $1 \mathrm{sec}$ delay.

We see that if the neutral pressure is too high when the RF is stopped, then a quicker decay results, most likely from scattering off of the neutrals. This occurs for pressures greater than $2.410^{-6}$ torr and delay times less than $250 \mathrm{~ms}$. If the neutral pressure is too low when the RF is shutoff, then there is a quicker decay, due to decreased ionization rate, which means a lower initial plasma density after the RF is stopped.

In fig. 4.7 a general correlation is shown between the gas-RF delay time and the characteristic decay time for a number of plasma shots. These are the times to decay to a 1 $\mathrm{MHz}$ frequency shift. These shots are taken with a starting $\mathrm{H}_{2}$ pressure of $9.010^{-7}$ tor, and we can see the trend towards faster plasma decay for a longer delay time. In this case the neutral density is low to begin with so the decay times do not decrease with short delay times. The overall decay times are much smaller as a result of the low initial pressure.

The only other investigation using this method was the effect of electrons emitted from the electron source on the plasma decay. The electrons caused the plasma to decay faster but there was a lingering signal from the RF-crystal that was most likely the result of the very low plasma density of the emitted electrons. 


\section{Gas-RF Delay and Plasma Decay Time Correlation}

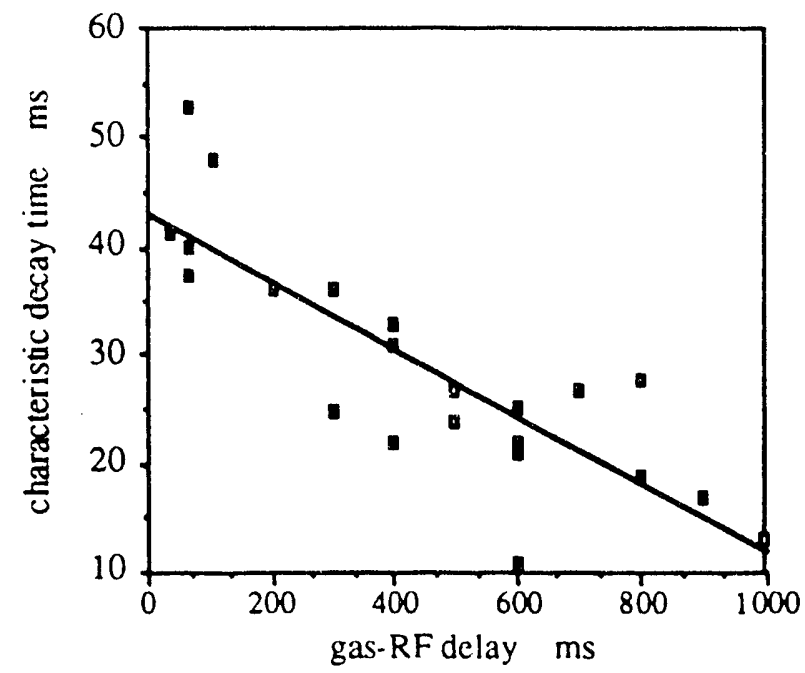

Fig. 4.7 The correlation between the gas-RF delay and the characteristic decay time of the plasma. The decay is to the $p=0$ mode. Gas pressure is in the $910^{-7}$ torr range.

\subsubsection{Density versus Time}

Density versus time plots were measured when the plasma was stable and reproducible. Fig. 4.8 shows data from five runs. All times were measured from the RF shutoff. Electron temperatures were 80 to $100 \mathrm{keV}$. What the plots all have in common is an initial fast decay then a slower decay. The fast decay is the cold electron component escaping. This occurs in 10 to $20 \mathrm{~ms}$. The slower decay is clearly the hot electrons slowly leaking out. The density of the hot electrons is in the $10^{7} \mathrm{~cm}^{-3}$ range. The best results are from run a. in which a high initial gas pressure is used. However, the $100 \mathrm{~ms}$ plus decay to the hot electron slow decay may be a result of the continued ionization of the still decreasing $\mathrm{H}_{2}$ gas. 

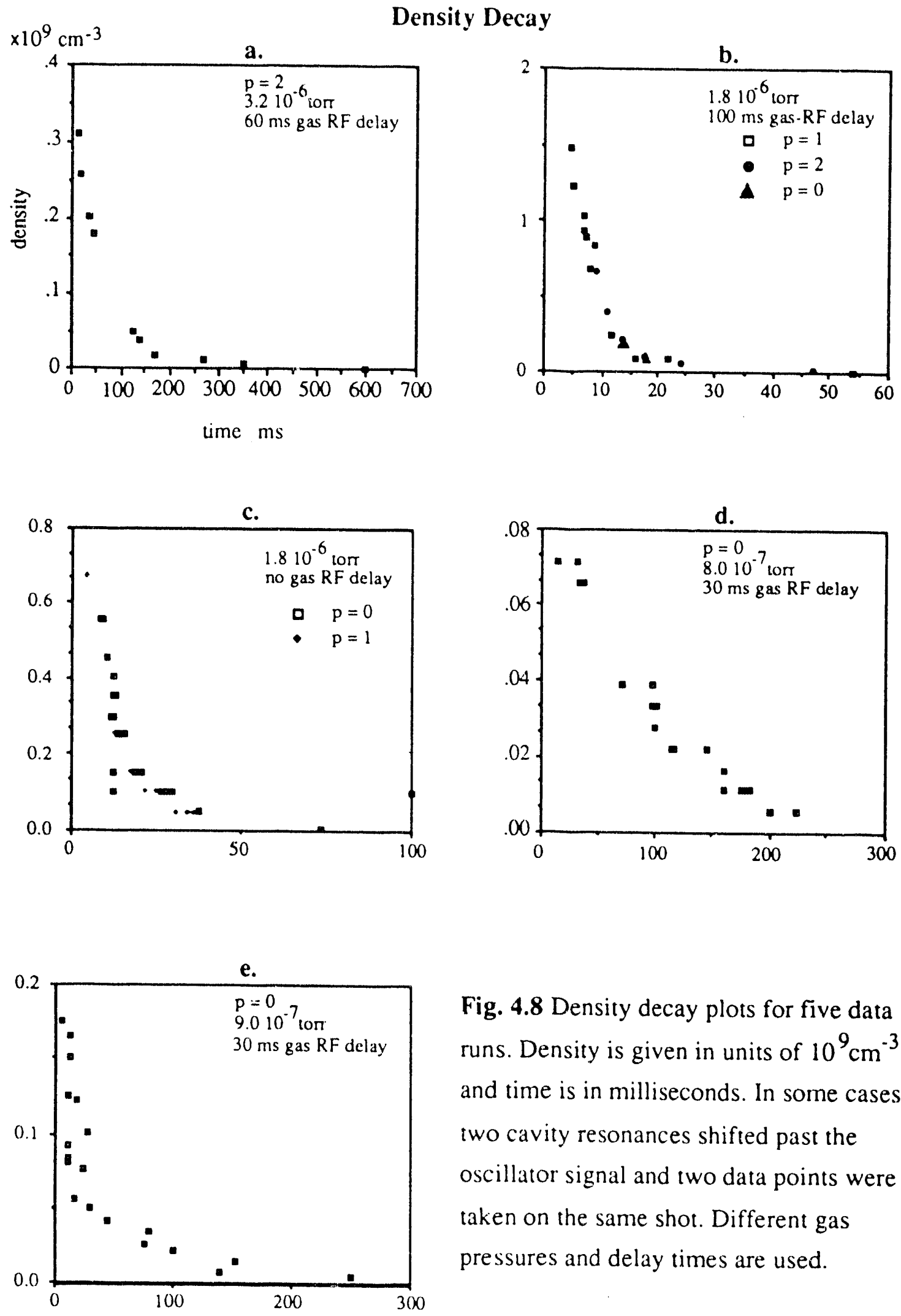

Fig. 4.8 Density decay plots for five data runs. Density is given in units of $10^{9} \mathrm{~cm}^{-3}$ and time is in milliseconds. In some cases two cavity resonances shifted past the oscillator signal and two data points were taken on the same shot. Different gas pressures and delay times are used. 
For runs $d$. and e. $x$-ray decay data were taken. The time scale of the x-ray decay appeared random during the data taking, ranging from 200 to $500 \mathrm{~ms}$. It is possible that there were some variations in the initial plasma conditions that led to random decay rates of the hot electron component. By assuming an exponential decay and the same initial density for each shot, then a 1/e decay time could be calculated from the one data point from each shot. In general the $1 / \mathrm{e}$ times became longer for data points taken later in the decay. This suggests a plasma with many temperature components that decay on different time scales. It was noticed that the variations in the $x$-ray decay time weakly matched the variations in the 1/e time.

Variations in the plasma decay could possibly relate to changes in the microwave power or frequency or changes in gas pressure. There appeared to be no correlation between the variation in gas pressure and $1 / \mathrm{e}$ time for shots in $\mathbf{d}$., but there was some for shots in e. When the pressure went above $9.210^{-7}$ torr for shots in e., there was a sharp rise in the $1 / \mathrm{e}$ time.

\subsection{Conclusions}

By this point I have learned how to create a hot electron plasma regularly in steady state. Hot distributions with temperatures of 80 to $100 \mathrm{keV}$ are typical with electrons in the tail reaching up to $1 \mathrm{MeV}$ in energy. These temperatures were obtained with only two channels of 50 watt microwave power. It still appears that the hot component is dominated by the cold component by approximately two orders of magnitude, i.e., $10^{9}$ compared with $10^{7} \mathrm{~cm}^{-3}$. These cold electrons typically have a temperature of $9 \mathrm{eV}$. The relatively low hot electron densities were not realized at the time, however, due to incomplete shift calculations.

Once the microwave heating is turned off the cold component disappears within a few tens of milliseconds. To obtain the highest hot electron density possible it is best to use a relatively high hydrogen gas pressure of 2.5 to $310^{-6}$ torr to make certain that the plasma 
remains stable during the steady-state condition before $\mathrm{RF}$ and $\mathrm{H}_{2}$ are stopped. Decreasing gas supply and drifting magnetron output resulted in varied initial plasma conditions. Allowing the gas to be pumped for 60 to $250 \mathrm{~ms}$ before the RF is stopped will allow the hot component to build up, as evidenced by the increase in $x$-ray output. In addition, it also minimizes the scattering of the hot electrons in the ensuing decay.

The best time to pulse a localized magnetic field at the midplane would be about 20 to $100 \mathrm{~ms}$ after the RF is stopped, depending on the cold electron density from continuing background ionization. This is when the bulk of the cold electrons has left and the hot electron density is at its highest. At this point, efforts were now redirected to creating the pulsed coil and its pulse generator. 


\section{Chapter V Phase Two Design}

The next stage of the experiment called for development of equipment to be used for novel purposes, but these components are based on familiar technologies. The first is a current loop to produce a fast rising magnetic field. The second is an electron beam to measure a transient potential.

\subsection{Pulsed Magnetic Field Technology}

The next step in the experiment was to design, build and test a system that could produce a fast localized magnetic field at the midplane of the mirror confined plasma. A peak magnetic field that could rise a few hundred gauss in only a few tens of nanoseconds was the goal. The requirements were stringent.

The pulsed coil was envisioned as a single turn loop placed at the midplane of the magnetic mirror. The loop would be driven by a slow charge, fast discharge pulse generator. Such a generator would be a flat transmission line with lo'v output impedance. 
The voltage pulse would last the length of time it takes the puise to propagate the length of the line. This would be a few tens of nanoseconds.

\subsubsection{Requirements}

The first step in the design is determining specific requirements. Two requirements for the pulsed coil are that it have a fast rise time and that it produce a steep magnetic gradient, which generally means a large peak magnetic field. The values for these quantities come from the theory and the configuration of the mirror confinement field. From the discussion in chapter two, a rise time of 10 gauss/ns would be appropriate. If the peak field is such that the combined field strength is equal to the mirror field, then this would mean an exclusion of all plasma electrons. Hence, the peak field should be about 400 gauss.

\subsubsection{Fast Rise Time}

To produce a fast rising magnetic field, high current must be driven through an inductive load very quickly. The field rise time is equal to the current rise time. (Retarded fields can be neglected here since characteristic dimensions, $10 \mathrm{~cm}$, divided by characteristic times in which the physical situation changes, $1 \mathrm{~ns}$, is still less than the speed of light.) In such a situation the current rise time is determined by $V / L, V$ is the voltage across the pulser coil and $\mathrm{L}$ is the inductance of the coil. This is true as long as $\mathrm{L}$ is much larger than the voltage source inciuctance. The aim then is to increase $\mathrm{V}$ and reduce $\mathrm{L}$.

Of course, the pulser coil must be placed inside the vacuum chamber, because such a fast rising pulse would not be able to propagate through the $1 / 8$ in. stainless steel wall of the vacuum chamber quickly enough. The implications this has for the $Q$ of the cavity will be discusses later.

The approximate necessary voltage around a strap loop can be calculated if the field rise time is to be 10 gauss per nanosecond. First, the current rise time is calculated. The field at the center of a loop of radius $r_{0}$ is 


$$
\mathrm{B}_{\mathrm{O}}=\frac{\mu_{0} I}{2 \mathrm{r}_{0}}
$$

An upper limit on the current needed is obtained by using the vessel radius, $r_{0}=13.3 \mathrm{~cm}$. A current rise rate of $212 \mathrm{amps} / \mathrm{ns}$ is required. The necessary voltage is $\mathrm{V}=\mathrm{L}(\mathrm{dl} / \mathrm{dt})$. Now an approximate inductive load is needed.

A simple formula to calculate the inductance of a single turn loop of width $w$ and radius $r_{0}$ is

$$
\mathrm{L}=.004 \pi \mathrm{r}_{\mathrm{o}}\left(\ln \left(\frac{8 \mathrm{r}_{\mathrm{o}}}{\mathrm{w}}\right)-\frac{1}{2}\right)
$$

where distances are in $\mathrm{cm}$ and $\mathrm{L}$ is in $\mu \mathrm{H}$. The inductance is only weakly dependent on the width of the loop. The inductance for a loop is modified in the presence of a conducting cylinder, but this will be neglected for now. An upper limit on the voltage is obtained by using the maximum $\mathrm{L}$, so again use $r_{0}=13.3 \mathrm{~cm}$. Let $w=1 \mathrm{~cm}$. The inductance is then about $700 \mathrm{nH}$. Using this figure a net loop voltage of $148 \mathrm{kV}$ is needed.

Decreasing the load inductance will be of help in decreasing the field rise time. The inductance will be determined by the loop dimensions. However, the magnetic field and the effect of the conducting wall should be considered as well, when deciding on the dimensions of the strap coil.

\subsubsection{Steep B Fiel' Gradient}

The magnetic field gradient and strength depend on the loop geometry, which, according to eq. 5.1, suggests making it as close to the plasma as possible. However, eq. 5.1 will be modified by the conducting wall. There is another factor determining the peak field strength. Once the greatest possible rate of current rise is achieved, then the peak field will be determined by the length of the voltage pulse. For our requirements, this means 
about a 40 ns pulse. In addition, the current should not be limited by the output impedance, $\mathrm{Z}_{0}$, of the voltage generator.

The peak output current possible from a generator source is $V / Z_{0}$. The maximum current drawn by the load is $(\mathrm{d} / \mathrm{dt}) \Delta \mathrm{t}$. To prevent possible source limitations we require $V / Z_{o}$ " $(d / / d t) \Delta t$. The restriction on $Z_{o}$ is then $Z_{o}$ " $160 \mathrm{kV} /(212 \mathrm{~A} / \mathrm{ns})(40 \mathrm{~ns})=19 \Omega$. This means that the output impedance should be $2 \Omega$ or less.

\subsubsection{Design of Pulser Coil}

\subsubsection{Coil Voltage}

The basic limitations on providing high voltage around the loop are the peak output voltage of the charging power supplies, load matching, and the difficulty in handling high voltage without breakdowns and flashovers. The power supplies obtained for use in charging the voltage generator were $10 \mathrm{kV}$ maximum. This is as high a voltage as is desirable for voltage handling purposes. Though it is far short of what is necessary.

An efficient way to increase the effective voltage around the circular path of the current is to break the conduction path up into eight segments. Then positive and negative voltage can be applied to the opposite ends of each segment. In this way the effective driving voltage is increased to $160 \mathrm{kV}$. This will meet the voltage requirement.

\subsubsection{Coil Dimensions}

There are a number of things to consider in the design of the pulser coil, such as the width and radius of the loop, the relationship of the loop to the wall and eddy currents, and how close the loop should be to the plasma. The loop inductance increases about linearly as $r_{0}$ increases. However, this behavior is modified when $r_{0}$ approaches the conducting wall. As the strap approaches the wall the inductance will reduce to zero as image currents in the wall become equal and opposite of the strap current. The sacrifice for lower inductance is 
that a lower magnetic field is produced. This will be considered in the following calculation.

It should be noted here that the vacuum spool designated to be modified with the pulser coil was of a standard size, unlike the vacuum sections used initially to build the plasma chamber. This means that the inner wall radius is slightly smaller at $12.38 \mathrm{~cm}$ instead of $13.33 \mathrm{~cm}$. This should result in slightly shifted resonant frequencies, but it should not affect the $\mathrm{Q}$ factor at all.

To decide on the loop dimensions, consider this model of the magnetic field produced by a loop in a cylinder

$$
\mathrm{B}(\mathrm{z})=\frac{\mu_{0} \mathrm{I}}{2} \frac{\mathrm{r}_{0}^{2}}{\left(\mathrm{r}_{\mathrm{O}}^{2}+\mathrm{z}^{2}\right)^{3 / 2}}-\frac{\mu_{0} \mathrm{I}_{\mathrm{w}}}{2} \frac{\mathrm{r}_{\mathrm{w}}{ }^{2}}{\left(\mathrm{r}_{\mathrm{w}}^{2}+\mathrm{z}^{2}\right)^{3 / 2}}
$$

$I_{w}$ is the image current created in the wall and $r_{w}$ is the effective radius of the image current. As $r_{o}$ approaches $R$, the wall radius, then $I_{w} \rightarrow I$ and $r_{w} \rightarrow R$. To approximate this behavior we can make these substitutions: $I_{w}=\frac{r_{0}}{R} I$ and $r_{w}=\frac{R^{2}}{r_{0}}$. This results in a modification of eq. 5.1

$$
\mathrm{B}(\mathrm{z}=0)=\frac{\mu_{\mathrm{O}} \mathrm{I}}{2 \mathrm{r}_{\mathrm{O}}}\left(1-\left(\frac{\mathrm{r}_{0}}{\mathrm{R}}\right)^{3}\right)
$$

As a double-check that this choice of $I_{w}$ and $r_{w}$ were acceptable, a computer code, XANTENA, was used to calculate the central field of a current loop in a conducting cylinder. The purpose of this code is to determine the parameters of antennae used for radiating power into plasmas. In fig. 5.1.a, the $1-\left(\frac{r_{0}}{R}\right)^{3}$ factor is plotted with some points from XANTENA. The match is reasonable. The slight offset could be due to the finite width of $1.27 \mathrm{~cm}(0.5 \mathrm{in}$.) used in the code, whereas eq. 5.3 is for filamentary current loops. 
The real interest is in the field gradient. The gradient of the unperturbed field is maximum at $z=r_{o} / 2$. Evaluating the gradient of eq. 5.3 at this maximum point gives

$$
\nabla \mathrm{B}_{\max }=\frac{3 \mu_{0} \mathrm{I}}{4 \mathrm{r}_{\mathrm{o}}^{2}}\left(\frac{4}{5}\right)^{5 / 2}\left(1-\frac{\left(\mathrm{R} / \mathrm{r}_{\mathrm{O}}\right)^{3}}{\left(\frac{1}{5}+\frac{4}{5}\left(\frac{\mathrm{R}}{\mathrm{r}_{\mathrm{O}}}\right)^{4}\right)^{5 / 2}}\right)
$$

The field depends on the current and the current depends on the inductance. Hence, the rate of rise of this gradient, $\frac{d}{d t}\left(\nabla B_{\max }\right)$, is determined by replacing I with $V / L\left(r_{o}\right)$. This behavior is shown in fig. 5.1.b where $\mathrm{L}$, and hence $\mathrm{I}$, is constant. Also, shown for comparison is $\frac{d}{d t}\left(\nabla B_{\max }\right)$ without the effect of the wall. The difference is the factor in large parenthesis in eq. 5.5

Now an expression for $L\left(r_{0}\right)$ is needed. Eq. 5.2 can be used for small $r_{0}$. For $r_{0}$ close to the wall radius $\mathrm{R}$, we can take a hint from the extra factor in eq. 5.5 and multiply eq. 5.2 by $1-\left(\frac{r_{0}}{R}\right)^{7}$ which is very similar to the factor in eq. 5.5. In fig. 5.1.c the loop inductance eq. 5.2 is shown with its modified version. In addition points calculated using XANTENA are shown. For both curves a width of $1.27 \mathrm{~cm}$ was used. There is reasonable agreement between the code calculation and this simple model.

Using this inductance in eq. 5.4 gives the rate of rise of the field gradient described in fig. 5.1.d. Here we can see that the gradient is dominated by the $1 / r_{0}{ }^{2}$ factor. Hence, the best possible choice for the loop is to make it as small in radius as possible.

As the loop radius moves in it begins to act like a limiter and starts to scrape off the outer plasma layer. Fig. 3.11 can be used as a guide for choosing a minimum pulser coil radius. At $r_{0}=10 \mathrm{~cm}$ the plasma density is half its peak value on axis. To make the coil much smaller would compromise the stability of the plasma and its ability to shield out external potentials. In this case the coil inductance is $350 \mathrm{nH}$. The rise rate of the magnetic gradient is about 1.5 gauss/(cm ns). The current rise time is 457 amps/ns. The magnetic field rise time is 11 gauss/ns. 
a. Loop Magnetic Field Inside a Cylinder

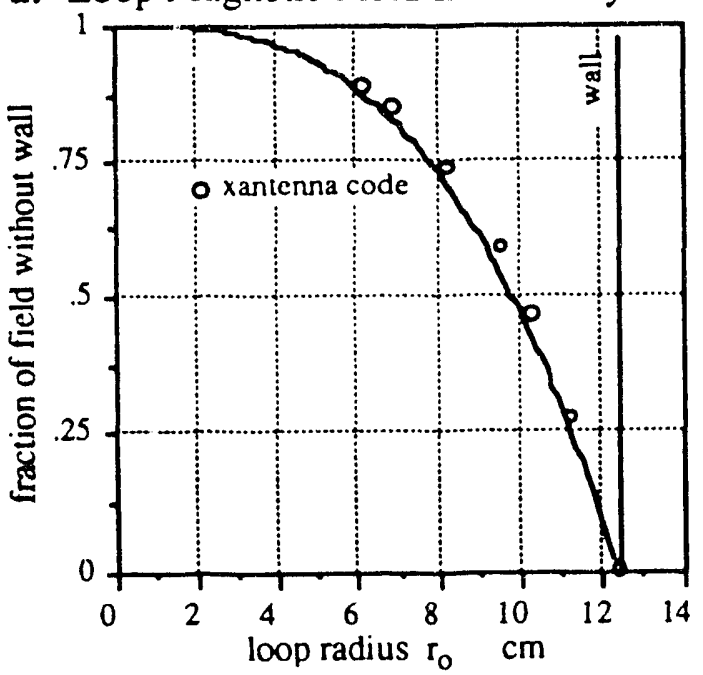

c. Inductance of Coil Inside a Cylinder

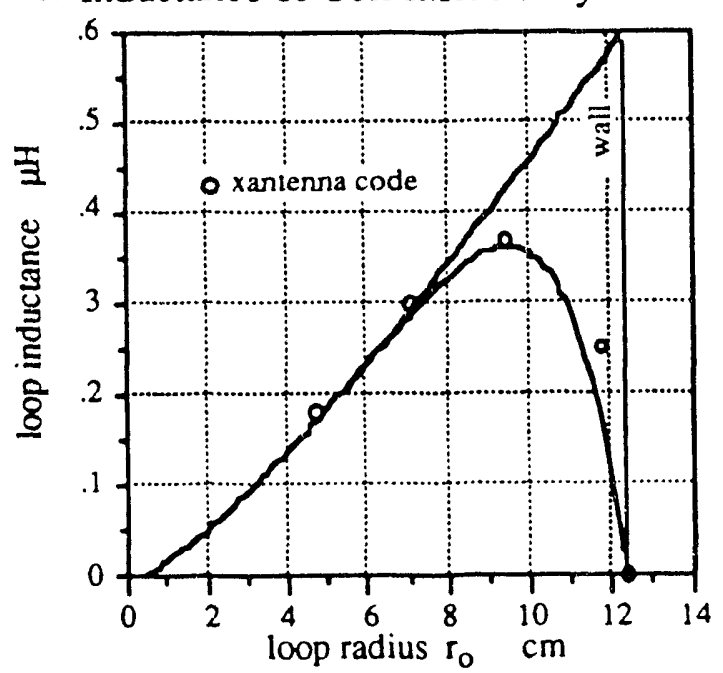

b. Magnetic Field Gradient vs. Loop Size

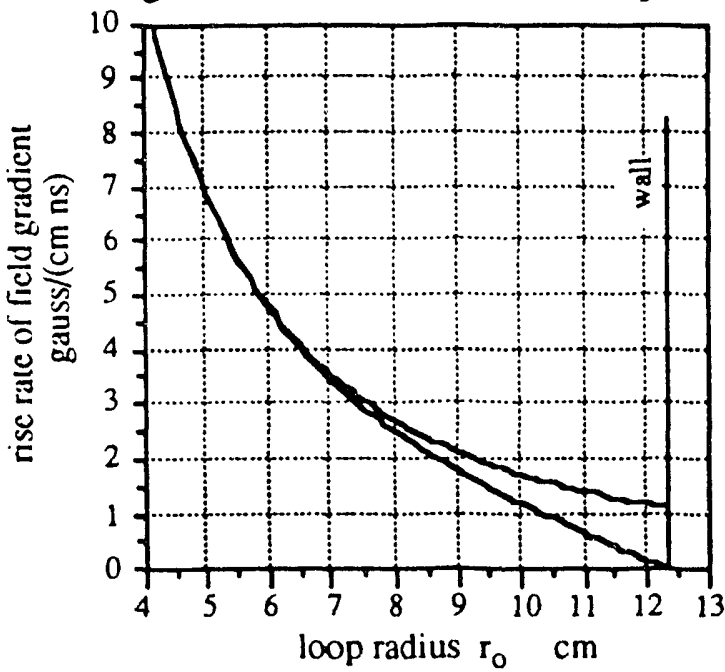

d. Magnetic Field Gradient vs. Loop Size

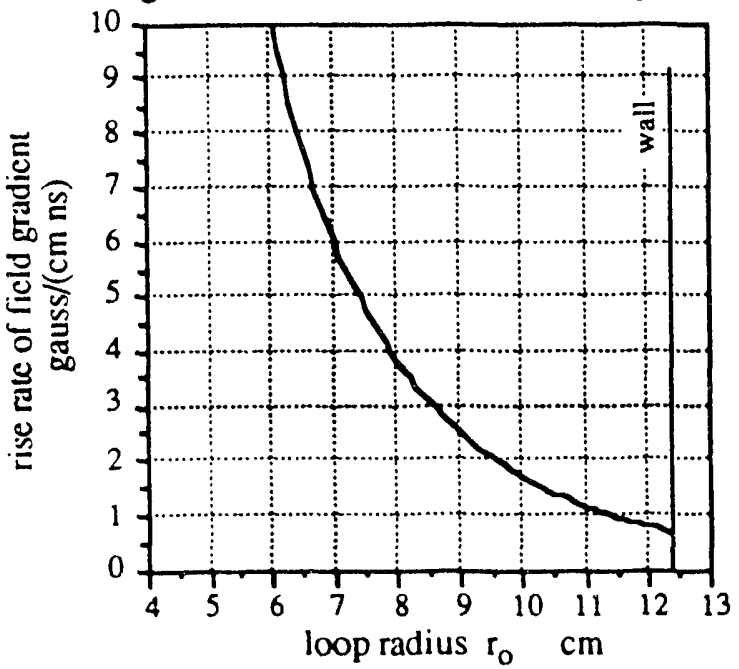

Fig. 5.1 Calculation of the linearly rising magnetic field gradient produced by a strap coil of width $1 \mathrm{~cm}$ inside a metal cylinder of radius $12.38 \mathrm{~cm}$. The gradient is determined for different radii of the strap coil. a. The magnetic field from a loop in a cylinder is plotted as a fraction of the field produced without the presence of the metal cylinder. $\mathbf{b}$. The field gradient is determined for a fixed current in the loop independent of loop size. An inductive load of $0.5 \mu \mathrm{H}$ is used. The upper curve is uncorrected for the wall currents. $\mathbf{c}$. This is the model of the inductance of a loop in a metal cylinder. d. Here the field gradient includes the effect of variable inductance on the loop current. 
The only other concern not considered so far is the effect of the added inductance due to the leads to each segment of the loop. The smaller the coil the longer these leads are. This may be significant in this case because the opposite polarity leads could not be placed close to each other. Due to allowances for the RF choke design in the feedthroughs, the ports were large enough that they had to be placed $3.8 \mathrm{~cm}$ (1.5 in.) on either side of the midplane so that they could fit in-line between the magnet rows. Nevertheless, a close in position of $r_{0}=10 \mathrm{~cm}$ was chosen.

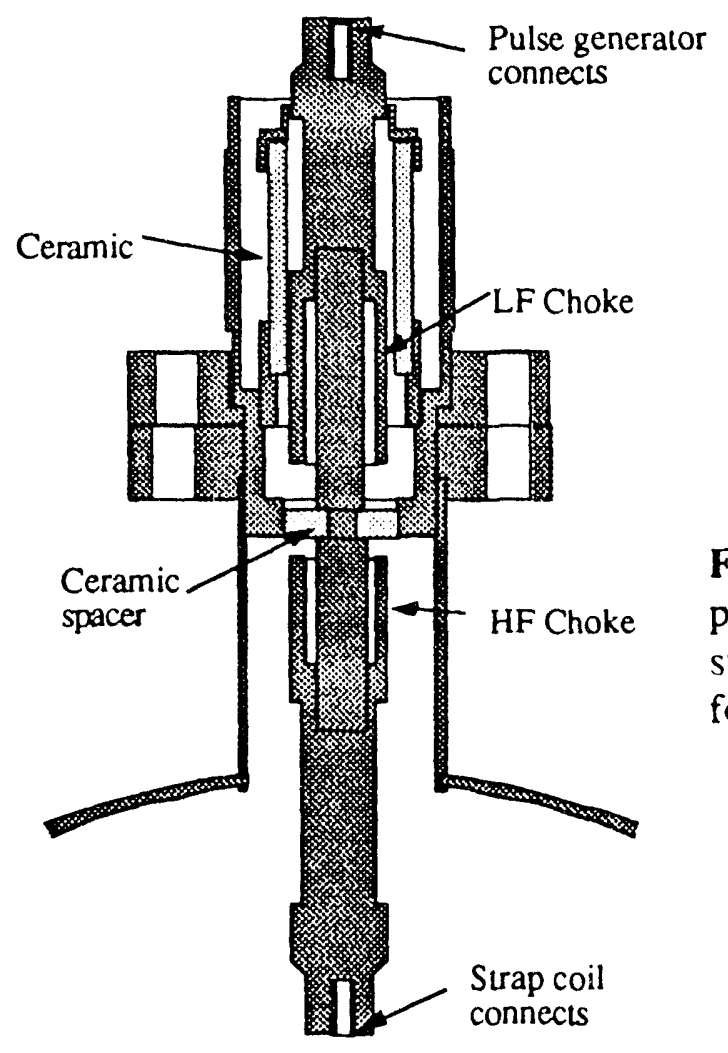

Fig. 5.2 A feedthrough port for the pulser coil showing the double choke structure that forms a high impedance for the heating microwave power.

\subsubsection{Feedthrough Design}

The need to reduce the microwave power conducted out of the cavity has led to an incorporation of a "choke" design into the strap coil feedthroughs. Fig. 5.2 shows the feedthrough design. The center conductor is a shaft and the choke is a cylinder of a certain length which surrounds the center shaft and is attached at one end. The cylinder length is equal to a quarter wavelength of the radiation to be stopped. The traveling field on the 
conductor surfaces inside the cylinder will be $\pi / 2$ out of phase at the end of the cylinder extension. This insures that there will always be a null field there. Hence, the microwave field will not be able to propagate out along the conductor. The cylinder extension length is $1.90 \mathrm{~cm}$ for the HF power $(3912 \mathrm{MHz}$ ) and $3.14 \mathrm{~cm}$ for the LF power $(2373 \mathrm{MHz})$.

The pulser coil and its feedthroughs are shown in fig. 5.3. The port flange size is $23 / 4$ in. Each pair of feedthroughs was placed 3 in. apart axially, each pair spaced every $45^{\circ}$ azimuthally. Each strap segment is in the shape of a "Z". It extends for 1.5 in. to the midplane, then arcs for $45^{\circ}$, reaching 1.5 in. over to the next feedthrough post. The separation of the posts and the extra axial stretch will add an extra inductance to the system. This extra inductance can be estimated from inductance formulae. An inductive impedance for the pulser coil is approximately $1 \mu \mathrm{H}$. This does not include the choke feedthroughs; however, they should have a small inductance. Considering this adjusted inductance, the maximum current rise rate is $160 \mathrm{amps} / \mathrm{ns}$, and the magnetic field rise rate is 3.8 gauss/ns.

An important question to consider once the pulser coil was installed: what is the effect on the $\mathrm{Q}$ of the cavity as well as the shifts in resonant frequencies? The smaller diameter of the center vacuum section should also affect the resonant shifts but not necessarily the $Q$. In both cases the resonance shifts were expected to be small because the volume of the additions is relatively small compared to the total volume of the cavity. From Hansen the frequency shift due to cavity perturbations is given by [17]

$$
\frac{\Delta \omega}{\omega}=\frac{1}{2} \frac{\int V}{\int B^{2} d V}
$$

where the integral over $\Delta \mathrm{V}$ is over the volume where there is now metal and the denominator is integrated over the entire cavity. E and B are the unperturbed fields. 


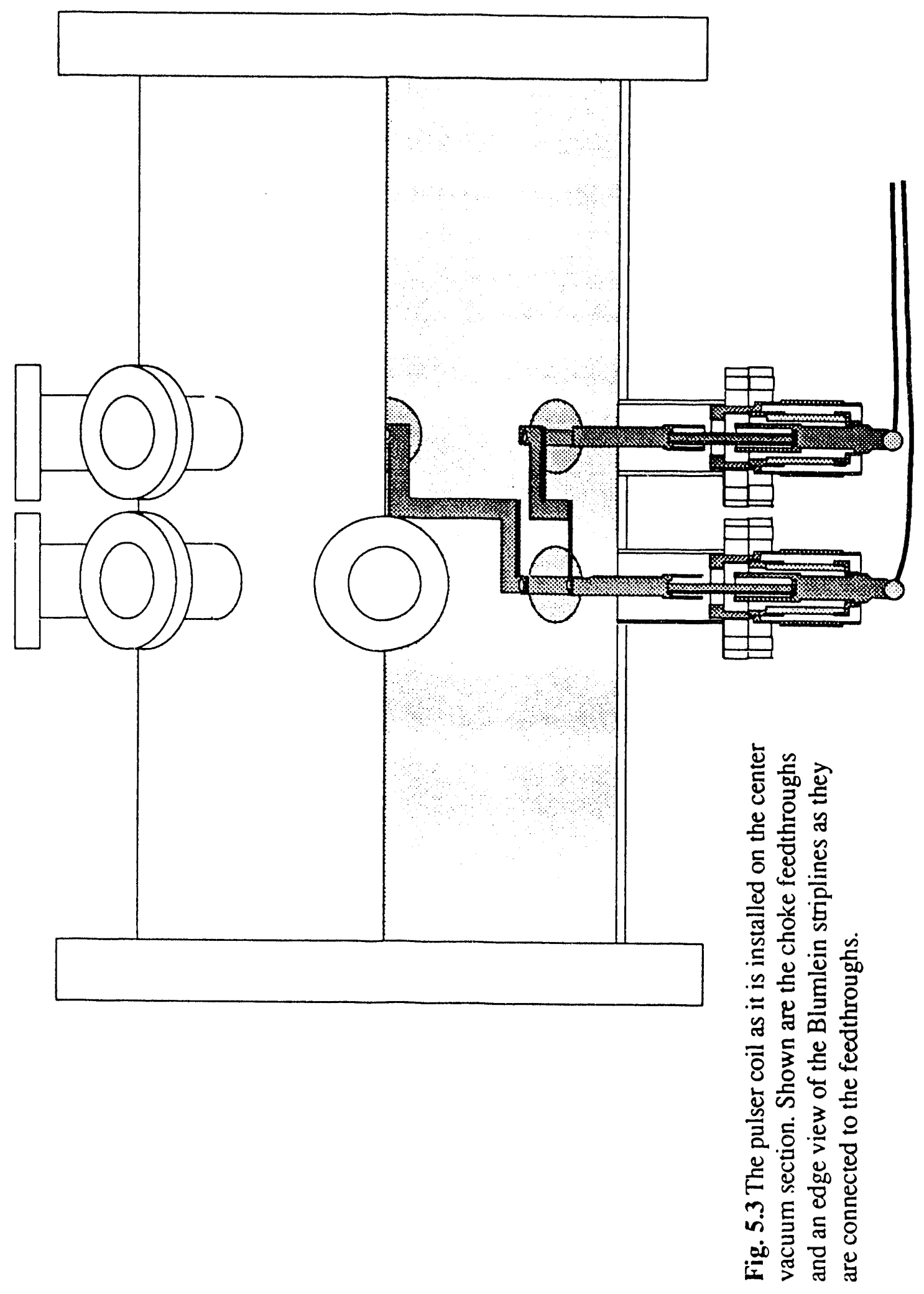


Performing this calculation for the strap coil the frequency shift is about $1 / 2 \mathrm{MHz}$ or less for the fundamental and the LF microwave frequencies. The effect of the smaller radius vacuum section leads to large shifts for even p modes. The shift is 39 and $28 \mathrm{MHz}$ for $\mathrm{p}=$ 0 and 2. It is only $3 \mathrm{MHz}$ for $\mathrm{p}=1$ because of its node at the midplane. For the $\mathrm{TM}_{1,1,20}$ mode the shift is calculated to be $38 \mathrm{MHz}$.

Once the pulser coil was installed in the vacuum chamber, tests were done to check the effect on the cavity $Q$ and resonances. The large expected shifts might have made the mode determination difficult. However, this time the tests took advantage of the 91 hole ventilation flange at the end of the cavity. These tests were done in air when the cavity was disconnected from the pump. A pick up antenna was then placed in any one of the holes, and by using many different holes, an idea of the $r-\theta$ dependence was deduced. This can determine the $\mathrm{m}$ and $\mathrm{n}$ mode numbers completely.

Fig. 5.4 gives a demonstration of the ease of mode identification by measuring the fields at the hole plate. In this figure the first two resonance modes were scanned. The lower six traces were taken from holes in a line, leading radially outward. The decrease in the peak amplitude agrees with the Bessel function $\mathrm{J}_{0}\left(\chi_{01} \mathrm{r} / \mathrm{R}\right)$ behavior. The top four traces were taken azimuthally at intervals of $20^{\circ}$ and at a radius of $6.7 \mathrm{~cm}$. They show that the fundamental modes are independent of azimuthal variation.

The first resonance plot was done at the fundamental frequencies. Fig. 5.5 shows this plot. The cavity field signals were detected from an antenna placed in the center hole of the end flange. Table 5.1 gives the frequencies, frequency shifts due to the modifications and measured $Q$ values of each of the first four peaks. The $p=1$ mode is the least affected because it has a field node at the midplane. The $\mathrm{p}=2$ mode is the most affected because it has a radial field at the midplane where the effective cavity radius is significantly smaller due to the pulser coil. The $Q$ values have been reduced by a factor of 2 to 3 . Considering that this reduction factor is for frequencies that the feedthroughs were not designed to choke out, it is acceptable. 


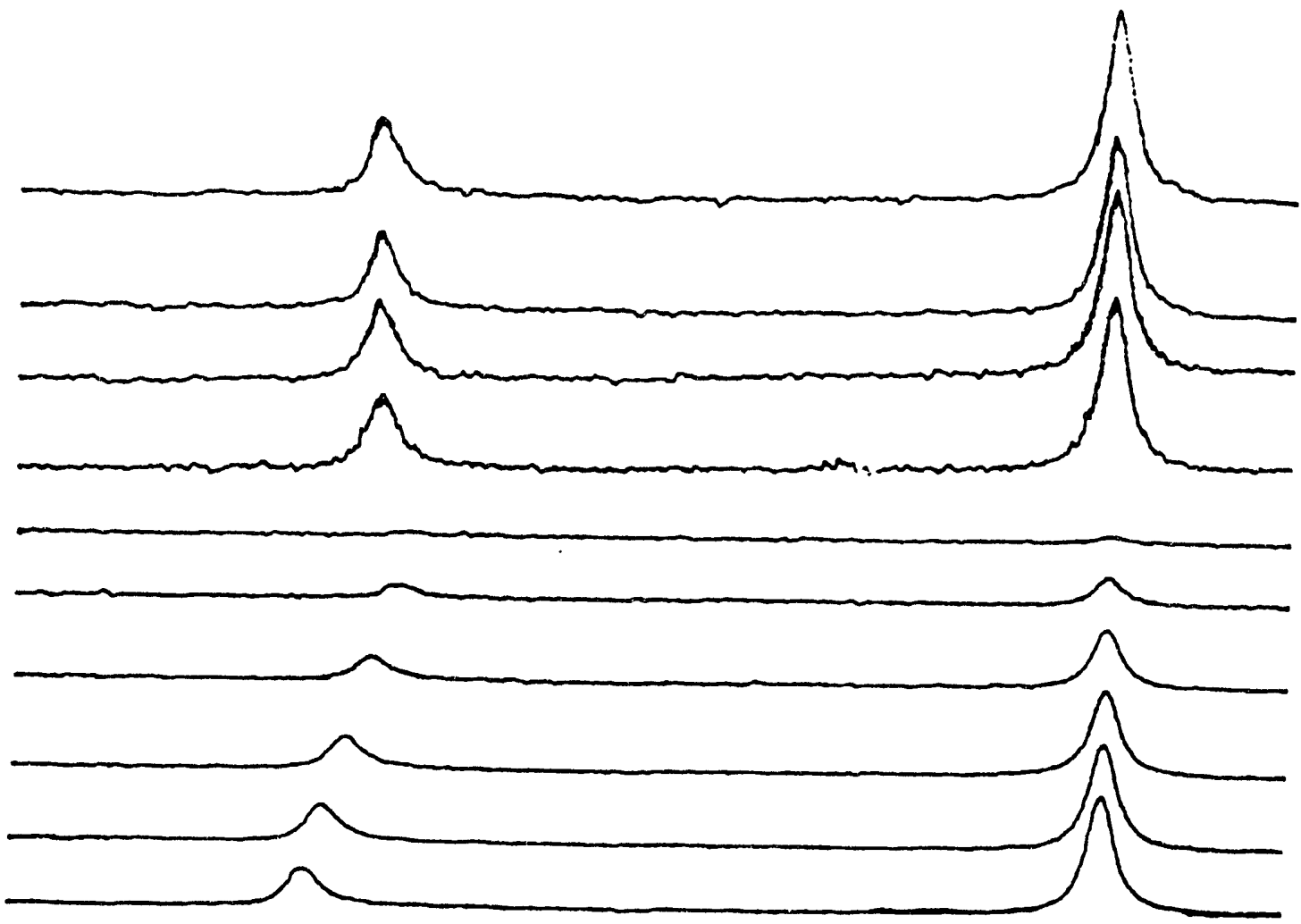

Fig. 5.4 A frequency scan from 864 to $871 \mathrm{MHz}$ showing the first two cavity resonances. The antenna that sensed the cavity fields was moved to different positions to determine an $\mathrm{r}-\theta$ dependence. Both resonances are $\mathrm{m}=0, \mathrm{n}=1$ modes.

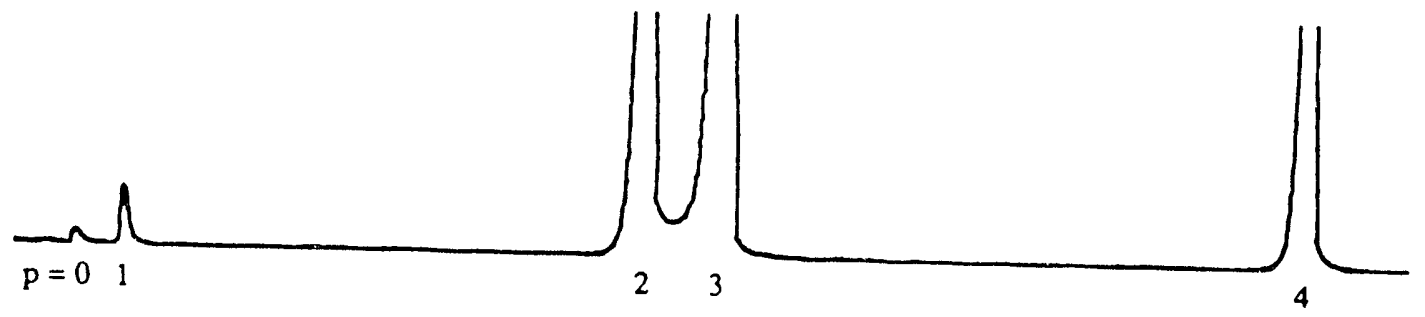

Fig. 5.5 A frequency scan of the first fundamental $T M m=0, n=1$ modes with the pulser coil included in the cavity. The frequency range is from $861 \mathrm{MHz}$ to $984 \mathrm{MHz}$. 
Table 5.1

Effect of Modifications on Resonances

\begin{tabular}{||c|c|c|c||}
\multicolumn{1}{c}{$\mathrm{p}$} & freq $(\mathrm{MHz})$ & $\Delta \mathrm{f}(\mathrm{MHz})$ & $\mathrm{Q}$ \\
0 & 865.5 & 5.1 & 3900 \\
1 & 869.8 & 3.2 & 4200 \\
2 & 915.7 & 32.6 & 2700 \\
3 & 922.4 & 13.8 & 2700
\end{tabular}

Unfortunately at higher frequencies the modifications introduce degeneracies in many resonances and increase the number of resonances. Identification by three simple mode numbers is not really possible. Fig. 5.6 shows the resonances in the range 2.3 to $2.4 \mathrm{GHz}$. Using the azimuthal traces as well, it appears that the most likely candidate for the LF resonance that was used previously has shifted by $6 \mathrm{MHz}$ to $2379 \mathrm{MHz}$. In such a situation when the mode density has increased and modes have shifted, the best way of finding a good heating frequency is by the trial and error method, as was done for the HF microwaves. As will be seen in the next chapter, the frequency $2424 \mathrm{MHz}$ works best for heating. From fig. 5.6 it looks like this mode may be an $m=0$ mode. Measurement of the $\mathrm{Q}$ values in this frequency range are from 2000 to 3000 . This is a factor of 2 to 3 less than in the unmodified chamber. As will be seen in the next chapter, this corresponds to a factor of 2 decrease in the overall emitted $x$-ray flux.

\subsubsection{Design of Voltage Generator}

The voltage generator is a stripline transmission line in which energy is stored capacitively and switched out with the low impedance characteristic of stripline cables. The length of the pulse will be determined by the length of the line. In standard lines and for matched loads, the load voltage is half the charging voltage. Blumlein transmission lines are an easy way of doubling the voltage across the load for the same charge on the line. Of 


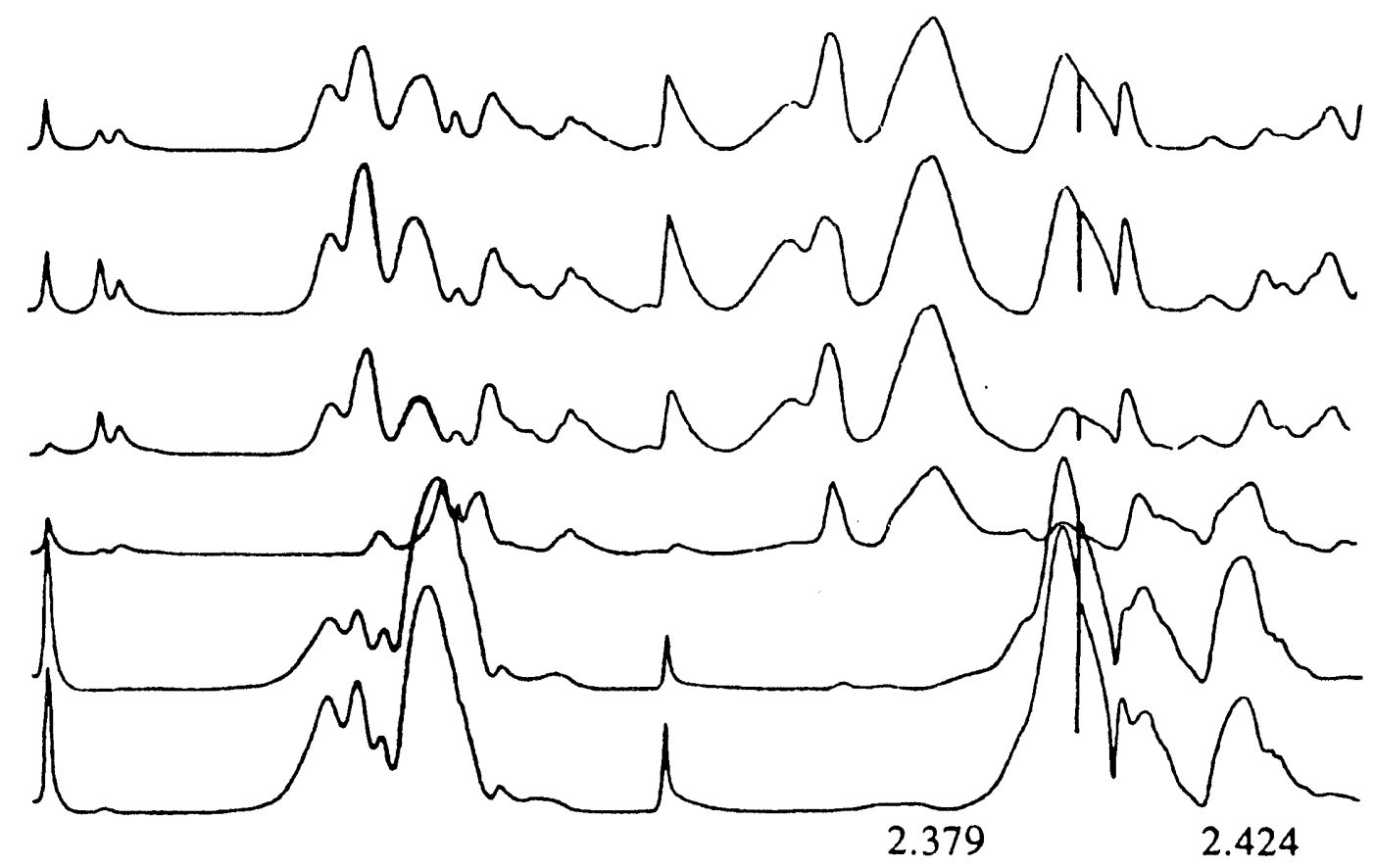

Fig. 5.6 A frequency scan from 2.25 to $2.45 \mathrm{GHz}$. The different traces are taken at increasing radial positions. Mode numbers can be inferred from the radial behavior of the various modes. $r=0$ is at the bottom.

course, since the load is an inductive one, the entire voltage will appear at the feedthroughs until the current begins to flow. However, the Blumlein design should still improve the pulse characteristics overall. In addition, there is the advantage of doubling the pulse length without doubling the physical length of the line.

\subsubsection{Blumlein Transmission Line}

The basic materials gathered to construct the striplines consisted of copper sheets $0.015 \mathrm{~cm}(0.006$ in.) thick and mylar insulating sheets that are $0.0076 \mathrm{~cm}(0.003 \mathrm{in}$.) thick. The copper sheets are in three widths. The center conductor is $5 \mathrm{~cm}(2 \mathrm{in}$. $)$ wide. The surrounding conductor is $10 \mathrm{~cm}$ (4 in.) wide. This conductor is charged. The outer grounded shielding conductor is $15 \mathrm{~cm}(6 \mathrm{in}$.$) wide.$ 
The hold off voltage of mylar is $5 \mathrm{kV}$ per $0.0025 \mathrm{~cm}(0.001 \mathrm{in}$.) of thickness. The mylar sheets are $0.0075 \mathrm{~cm}(0.003$ in. $)$ thick. It was decided that two sheets should be the minimum used to prevent burn-throughs at weak spots in the mylar. The Blumleins could then be expected to hold off $30 \mathrm{kV}$ which provides a safety margin if the charge is to be only $10 \mathrm{kV}$.

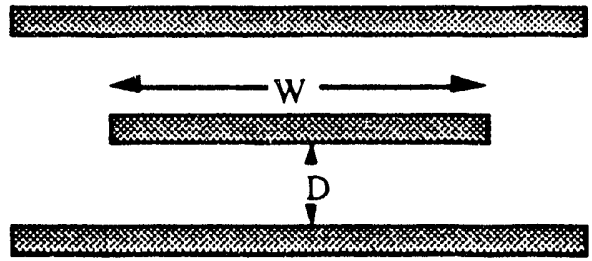

Fig. 5.7 Dimensions of a standard strip transmission line.

To calculate the characteristic impedance of such a voltage generator, consider the basic stripline transmission line shown in fig. 5.7. The capacitance per unit length and inductance per unit length of this line are

$$
\begin{gathered}
C=2 K \varepsilon_{\circ} \frac{W}{D} \\
L=\frac{\mu_{0}}{2} \frac{D}{W}
\end{gathered}
$$

$\mathrm{K}$ is the dielectric constant of the medium between the conductors. For mylar $\mathrm{K}=2.5$. The impedance per unit length is then

$$
\mathrm{Z}_{\mathrm{o}}=\sqrt{\frac{\mathrm{L}}{\mathrm{C}}}=\frac{1}{2 \sqrt{\mathrm{K}}} \frac{\mathrm{D}}{\mathrm{W}} \mathrm{Z}_{\mathrm{f}}
$$

where $Z_{f}$ is the impedance of free space, $377 \Omega$. To make a Blumlein generator, the center conductor separated into two sheets and another conducting strip is placed between them. The center strip becomes two intermediate strips so that now there is a transmission line within a transmission line. The load is attached between the inner and outer strips. 
To minimize power reflections it is best to have the inner line impedance equal to the outer line impedance. This is easily done by using the same spacing to width ratio in both parts of the line. The inner line then determines how low the impedance will be because the rninimum insulation that is necessary is used in this section. With two sheets of mylar ( 0.003 in. each) and a two incn wide inner conductor the line impedance is about $0.36 \Omega$. The Blumlein impedance is the sum of the inner and outer line impedances. Hence, $Z_{0}=$ $0.72 \Omega$. This certainly mects the requirement that $Z_{0}$ be less than $2 \Omega$ derived earlier.

Operation of the generator begins by charging up the $10 \mathrm{~cm}$ wide conductor. The pulse to the load is then delivered by shorting the charged stripline to the outer conductor which is grounded. In our case, the charge s.tip will be shorted to the charge strip of the oppositely charged pair. Both lines will $L_{2}$ connected to a virtual ground. The pulse propagates duwn the outer line to the luad, and the pulse on the load begins. The voltage on the load remains high until the pulse front propagates down the inner line, reflects at the end of the center strip and returns to the load. In a matched load case, the voltage on the load would be over by the time the last voltage front travels along the outer line to the starting point. Then the shot is over.

The speed of the pulse front in the transmission line is $\mathrm{c} / \sqrt{\mathrm{K}}=19 \mathrm{~cm} / \mathrm{ns}$ for mylar. Our Blumlein transmission lines were made three meters long, so the propagation time is $15.8 \mathrm{~ns}$, and the entire pulse will be $32 \mathrm{~ns}$. A $15 \mathrm{~cm}$ wide fiat line that is much longer than three meters would be unmanageable.

Two sets of Blumleins were eventually made. The first set was used in the bench test set-up. The second set implemented improvements over the first set. This set was used in the actual Mirrortron experiment. In both cases, the lines were designed to lead from their connections at the midplane feedthroughs along the length of the vacuum vessel between the permanent magnets and the electromagnets. The lines were stacked together in oppositely charged pairs. When the eight line pairs emerged from inside the end 
electromagnet, they entered a support structure that carried them to a merging point where a tack switch was located.

There were two concerns about the performance of the Blumleins. The first concern was maintaining the minimum spacing between the conducting sheets. Any stress on the lines would cause the sheets to buckle and separate, resulting in locally high impedance. The second was the possibility of voltage breakdown.

There were two solutions to the problem of the separation of layers. The solution to the problem of buckling was to press the line between two thick boards or plates where the lines lay flat. On the first set of lines, this was implemented on the section of line between the electromagnets and the switch. The support structure served this purpose. It consisted of one half inch thick phenolic boards that were bolted together. Each Blumlein pair was sandwiched between a set of boards. This solution was only partially implemented inside the magnets toward the feedthroughs. In addition, the feedthroughs were $7.5 \mathrm{~cm}$ apart, however, the Blumleins were all the same length. This meant that the bottom line in each stacked pair made a loop so that it would be effectively shortened. This was a good spot for buckling.

These problems were solved in the second set of lines first by making the bottom line $7.5 \mathrm{~cm}$ shorter than the top one. This meant that the negative pulse would arrive at one end of each strap coil segment about 0.4 ns before the positive pulse arrived at the other end. It was thought that this would not result in any serious problems. Also, a rack was made of quarter inch aluminum plates for the purpose of supporting and pressing the lines inside the electromagnets.

The second solution to layer separation was to use double sided tape between each layer of the Blumlein along its entire length. Double sided tape was used only sparingly in the first set of lines with the idea of making them more flexible. The addition of the tape added extra thickness to the Blumleins so that its effective impedance would be increased. The thickness of the tape was determined to be $0.005 \mathrm{~cm}$. The inner line impedance was 
calculated to be $0.71 \Omega$. In the outer line, a mylar sheet of $0.019 \mathrm{~cm}$ was used instead of two sheets of $0.008 \mathrm{~cm}$, as in the first set of lines. The outer line impedance was then 0.64 $\Omega$, and the total impedance $1.35 \Omega$.

The possibility for voltage breakdown is greatest at the edge of the charged strips, especially when the lines buckle and create air gaps in which a corona discharge could form. The cross-section of the final Blumlein design is shown in fig. 5.8. This shows that there is a $3 / 4$ in. wide band of mylar insulation on either side of the 2 in. center conductor, and again for the 4 in. charged conductor. In air, a $3 / 4$ in. gap could hold off over $25 \mathrm{kV}$, however, we have field enhancement effects from the foil edge, and insulator surface flashover effects. The use of double sided tape was helpful in that it eliminated air gaps. In addition, a half inch strip of Kraft paper was placed along the conductor edges. Kraft paper is not as insulating so that it tended to disperse any charge buildup before it led to a flashover.

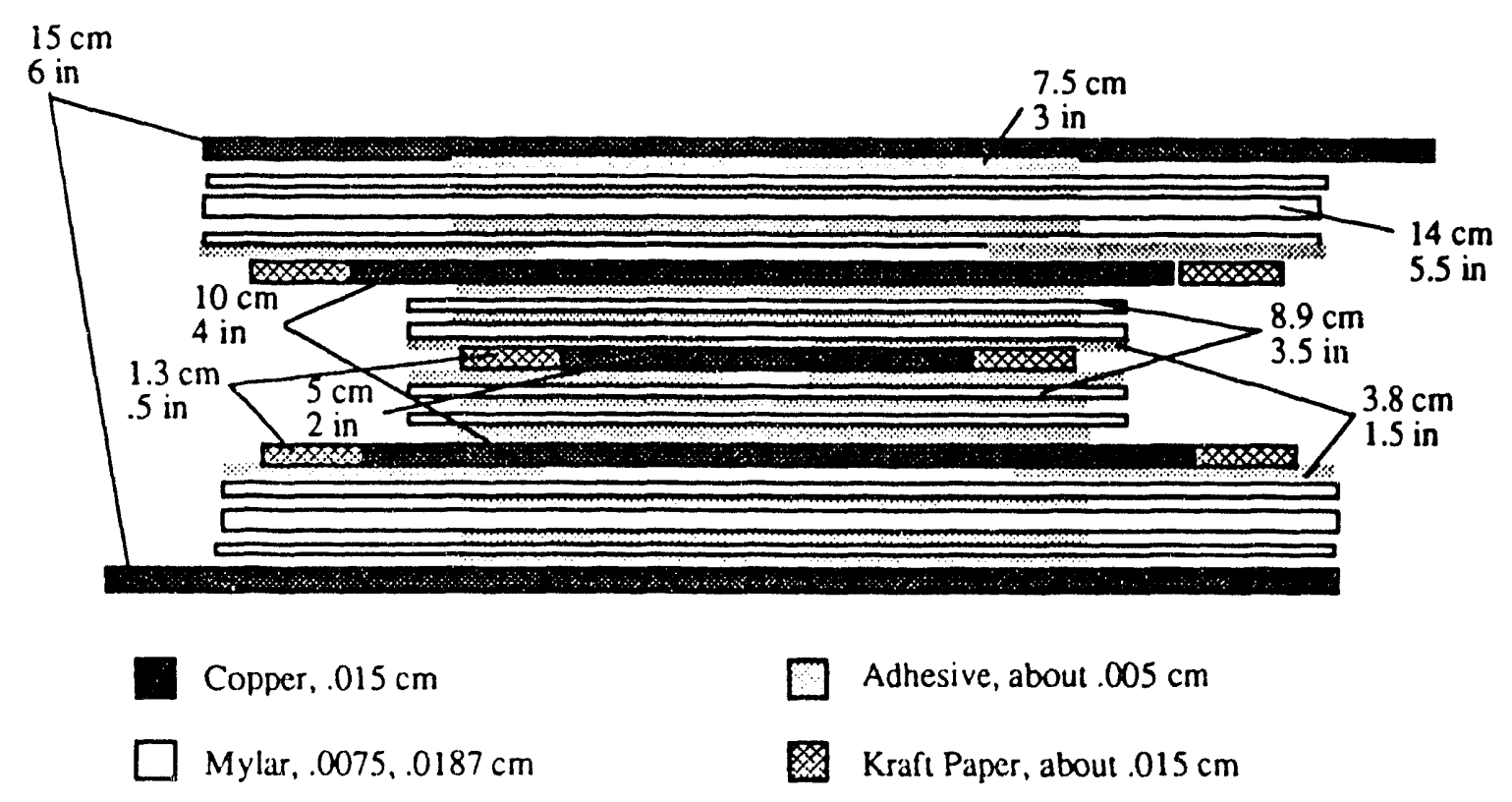

Fig. 5.8 Detail of the cross section of the Blumlein transmission line. The half inch tabs on the 6 inch, $15 \mathrm{~cm}$ wide outer copper sheet are to be folded over. 
An estimate of the energy stored in this voltage generator is obtained from eq. 5.7.a.

The stored energy is

$$
\varepsilon=\frac{1}{2} C V^{2} \text { (length) }
$$

This means that 2.3 Joules are stored in each Blumlein generator with $3.7 \mathrm{~J}$ in the switch region. The total stored energy is 41 Joules. Fig. 5.9 gives a schematic view of how this voltage generator will deliver this energy to the pulser coil.

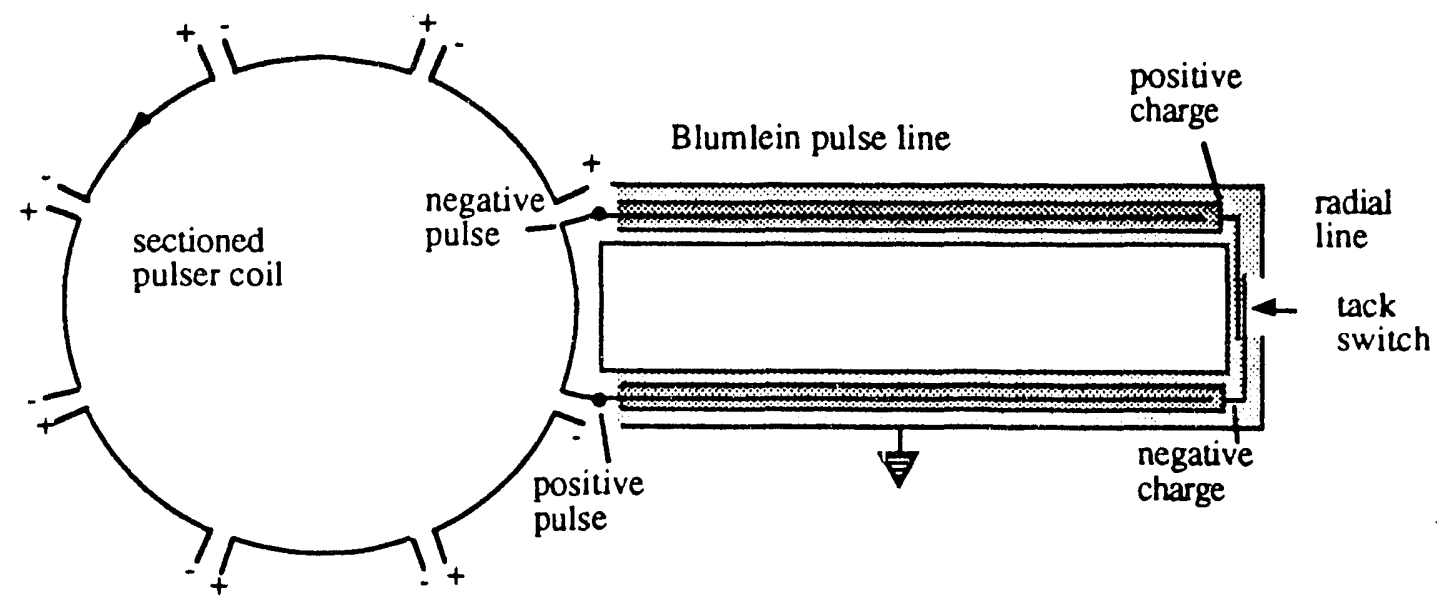

Fig. 5.9 Schematic view of the pulser coil and the Blumlein generator.

\subsubsection{Switch}

The purpose of the switch is to initiate the voltage pulses to the leads on the strap coil segments. It must have an impedance small compared to $125 \mathrm{nH}$, which is the portion of the strap coil load driven by one Blumlein pair. It must simultaneously start a pulse in each of the 16 Blumlein pulse generators. If the switch is to be mechanical, this means that there can be only one switch and that the 16 lines must be tied together. Otherwise the timing of pulses would have presented an impractical problem. The best design for this is where the lines converge radially and the switch is located at the center. Of course, radial lines have 
different impedance characteristics from a straight transmission line, and the implications of this will be discussed later.

Since the impedance of the switch must be low, a tack was utilized. The tack switch is a sharpened rod that is driven through a thin insulator between positive and negative charge sheets to close the gap and begin the current flow. It is a reliable and consistent switch that works for $10 \mathrm{kV}$ and has an inductance of less than $1 \mathrm{nH}$ [18]. In addition, this type of switch is attractive because of its simple design and it is easy to use. One disadvantage is that the insulation is destroyed and must be replaced for every shot. The repetition rate is not important here, so this does not present a problem.

Fig. 5.10 describes the design of the radial transmission line and the tack switch at its center. The radial line and the Blumleins could not be made out of one single piece, so the ends of the straight lines are sandwiched into the edges of the radial line. Near the end of the Blumlein transmission line, the $5 \mathrm{~cm}$ ( 2 in.) center conductor ends, then the charged strips become pressed together. A standard stripline, as in fig. 5.7, is then formed. These lines are then bent inward $90^{\circ}$ after emerging from between the phenolic boards. The ends of these lines interweave with the layers of the radial transmission line. The edge of this line is a circle of $20 \mathrm{~cm}$ in radius. The outer grounded strips of the Blumlein stop at this point, and the charge strips of each Blumlein pair continue inward to overlap two foil discs.

The circular radial line is pressed between two $3 / 4$ inch Plexiglas plates. The top Plexiglas pressure plate and the top ground plate have a $12.5 \mathrm{~cm}$ ( 5 in.) diameter hole at their centers where a removable tack driver, shown in fig. 5.11 , could be placed. This tack driver is mounted on a phenolic base that fits inside the hole in the pressure plate. Clamps mounted on the pressure plate could then be tightened to push the tack driver into place. A replaceable sandwich is made by layering a $12.5 \mathrm{~cm}$ (5 in.) mylar disc, a $10 \mathrm{~cm}(4 \mathrm{in}$.) copper disc, and a $7.5 \mathrm{~cm}$ ( $3 \mathrm{in}$.) mylar disc. This disc is then placed into the hole and the tack driver clamped into place over it. When the tack was launched, it pierced these three layers. 


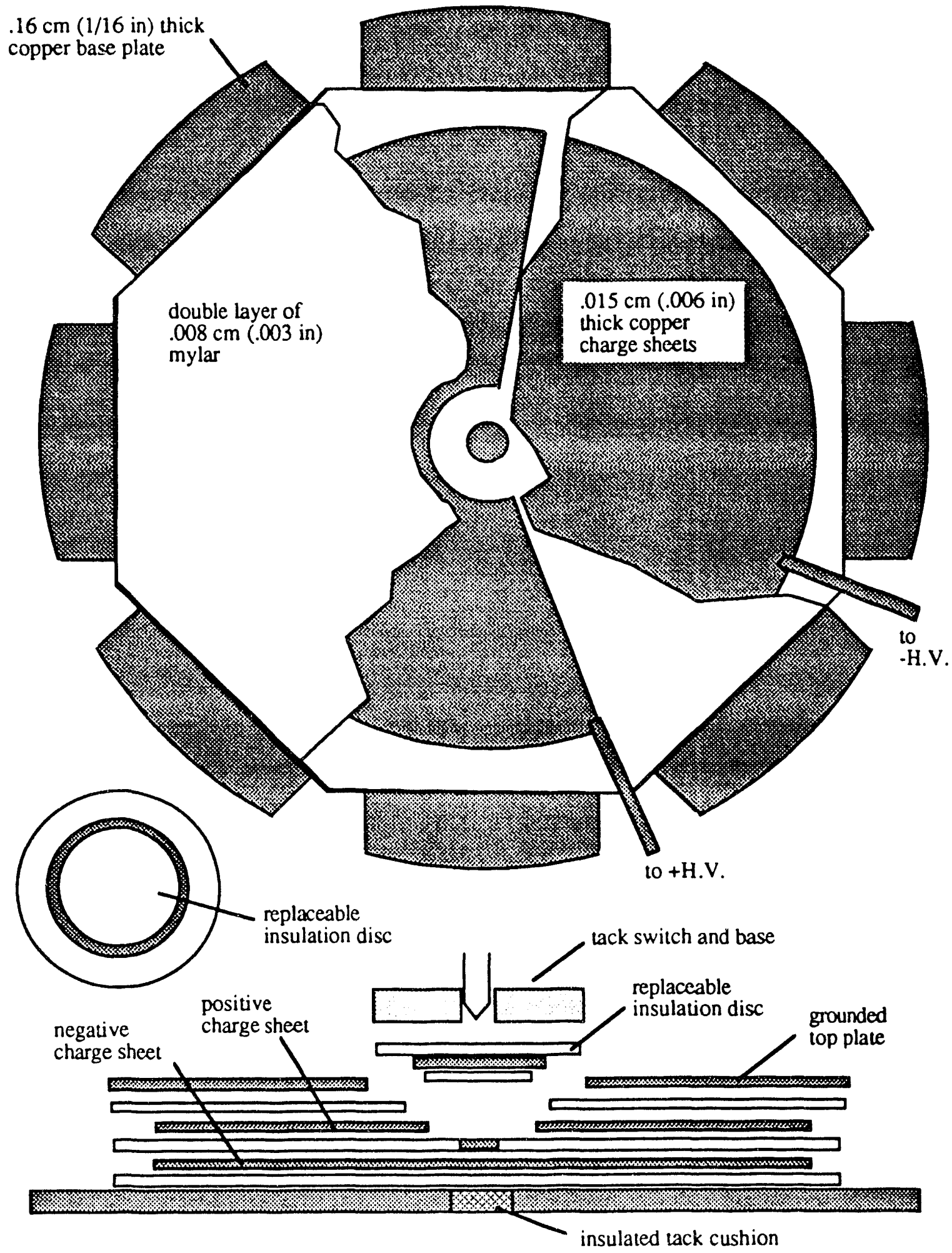

Fig. 5.10 The tack switch and the radial transmission line portion of the pulse generator. Eight pairs of Blumlein transmission lines converge from the outer edges and connect to the charge sheets. 
There was a concern that the replaceable section might compromise the voltage holdoff capability of the pulse generator system. One solution to this problem came from the use of silicon grease placed on the insulation of the disc. This increased the hold-off capability in this portion of the switch. It also helped to hold the replaceable disc in position until the tack driver was clamped in place.

The insulation between the two charged discs as well as between these discs and the ground plates consists of two sheets of $0.0075 \mathrm{~cm}$ thick mylar. The maximum voltage difference between the charged discs would be $20 \mathrm{kV}$ and the hold off voltage for the insulation is about $30 \mathrm{kV}$. This represents a decreased safety factor over that of the Blumleins. However, should there be a burn-through or flashover, a new radial switch line could be built and replaced easily, unlike the Blumleins.

The tack iself is made of tungsten welding rod. It is mounted in an insulating block on the plunger so that a voltage pulse is not transferred to the driver assembly. The plunger is driven forcefully by high air pressure behind the plunger. The plunger is locked in place by three ball bearings. The ball bearings are held in place by the sleeve. The sleeve position is controlled by a solenoid. When the solenoid is momentarily energized, the sleeve is pulled back, and the ball bearings move out releasing the plunger, which is then driven forward. A control box containing air pressure valves is manually operated to redirect the pressure flow to push the plunger back and reset it in a locked position so to recharge it.

During bench testing, the tack was fired manually. However, during plasma operations, control over tack firing was more precise. The control box of fig. 3.17 was modified by adding one more potentiometer and delay unit with control relay to operate the tack driver solenoid. It was then a matter of trial and error to find out what time scales the mechanical tack operated on. 


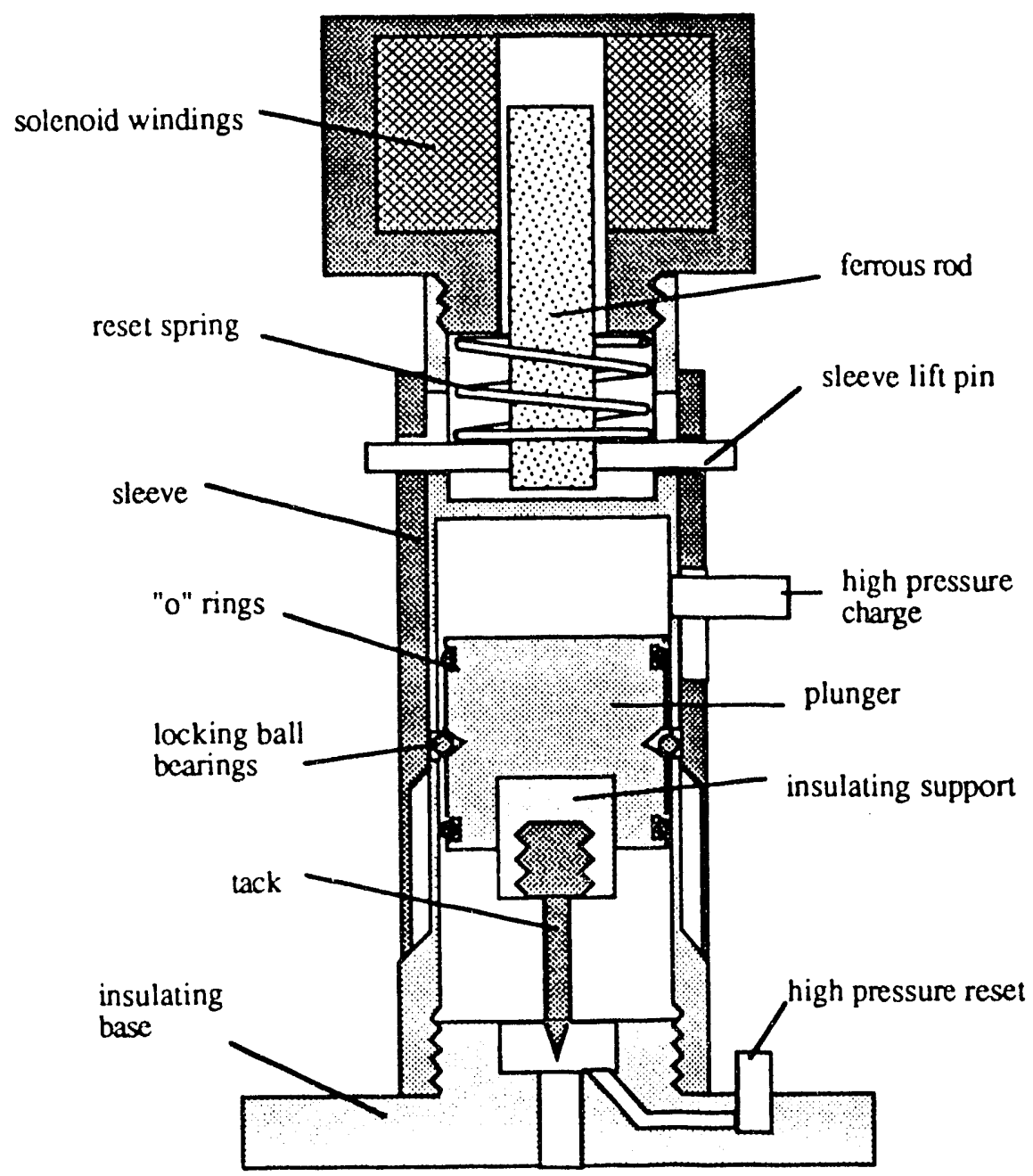

Fig. 5.11 This is the tack driver used to close the connection between two oppositely charged copper sheets, and thus begin a voltage pulse.

The charged discs in the switch section are charged by tabs that lead out between the pressure plates where they connect to a cable going to a resistor box. This box contains a $15 \mathrm{M} \Omega$ charging resistor that prevents an overload on the supplies when the charged sheets are shorted together. This results in a charge up time of about one minute. Also in this resistor box is a pair of voltage dividers that indicate the actual voltage on the charged sheets. The total resistance in each divider is $1000 \mathrm{M} \Omega$.

The switch section of the pulse generator is essentially a radial transmission line. Radial transmission lines have a characteristic impedance given by 


$$
Z_{r}(r)=\frac{Z_{0}}{2 \pi \sqrt{K}} \frac{D}{r}
$$

The impedance varies inversely with distance from the center. Hence, the impedance at the tack location can be very high. At some point, as the tack pierces the insulation, there will be a spark. All the charge stored in the transmission lines will travel in this current arc. The outer radius of this arc will define the peak impedance in the radial line. A lower limit may be $r \approx D=0.018 \mathrm{~cm}(0.007 \mathrm{in}$. $)$. In this case $Z_{r}=38 \Omega$. An upper limit may be the radius of the tack at $r=0.079 \mathrm{~cm}(0.031$ in. $)$ so that $Z_{r}=8.5 \Omega$.

To understand what effect this behavior might have on the voltage pulse propagating in the line, a computer code was written to simulate a pulse front heading outward from the center of a radial line. The radial distance out to $20 \mathrm{~cm}$ was divided into 100 zones. At the interface between each zone transmission and reflection coefficients were calculated for outward and inward propagating wave fronts. Eq. 5.10 was used to calculate these coefficients except in the last centimeter, where a linear interpolation to $0.4 \Omega$ at the edge was used since this is where the radial line joins the straight transmission lines. An initial normalized wave front was placed at the center, which was then free to propagate outward. The result is shown in fig. 5.12.

The effect of the radial switch section on the voltage generator is to blunt the square voltage front and to give it a damped ringing. The oscillation period is about $4.5 \mathrm{~ns}$ and the oscillation is significant for mast of the length of the pulse. Hence, the current in the pulser coil and so the magnetic field will not necessarily grow linearly but it will still increase positively.

\subsubsection{Bench Test}

The purpose of the bench test was to measure the magnetic field produced as a function of time, and to compare it to the expected field. To make this comparison an 
The Front of the Voltage Pulse

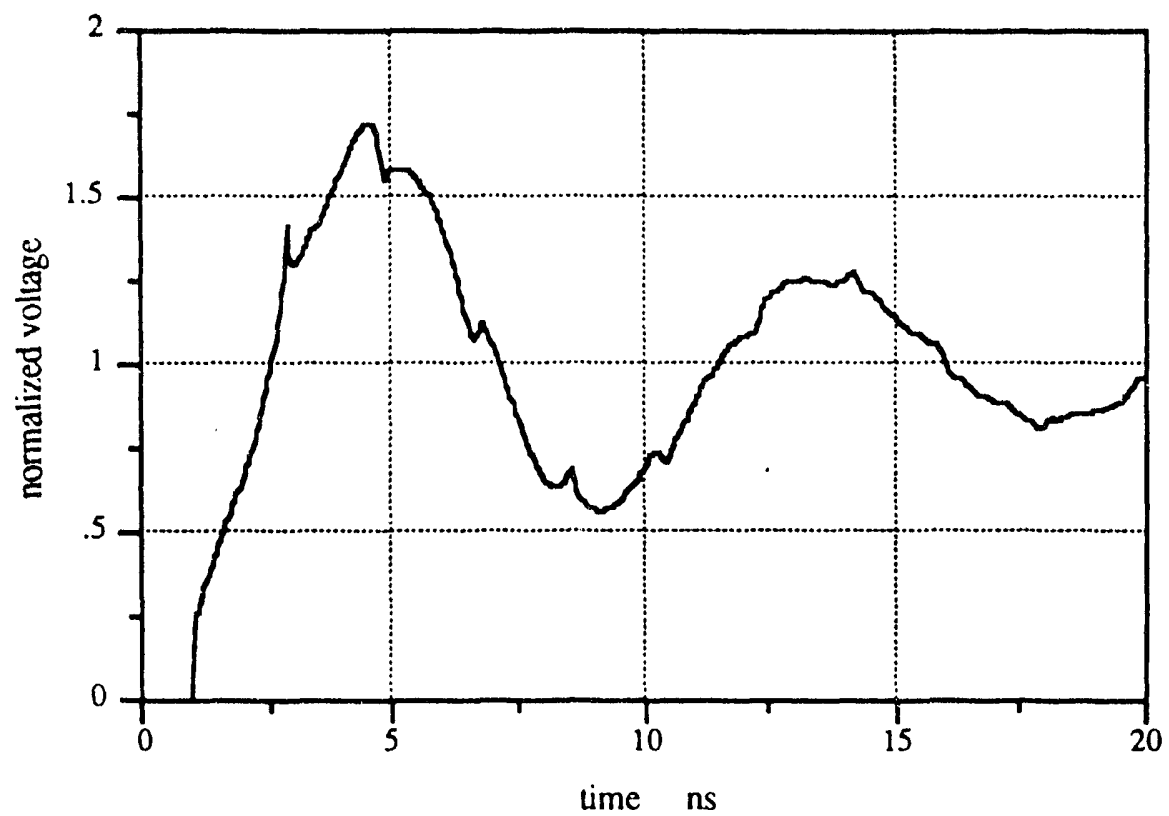

Fig. 5.12 A computer simulation of the voltage front as it emerges from the radial switch region. At $t=0$ the tack switch closes. The pulse takes about a nanosecond to reach the edge of the radial line.

experimental measurement of the coil inductance was made. Another reason for this test was to check the general operation of the pulse generator and make improvements where possible. Such improvements included grounding the floating pulser coil through an impedance large compared to $\mathrm{Z}_{0}$, and signal noise reduction. The noise radiation from the tack switch spark was a serious problem. In addition, these tests helped improve the second version of the Blumleins. Variations on the radial switch design were possible because of the ease with which it could be accessed.

\subsubsection{Pick-Up Loop}

The most important measurement was the field rise time. This measurement was made with a pick-up loop placed at the center of the pulser coil. If the area surrounded by the 
loop is small then the magnetic field can be considered consiant across the area and the induced signal at the ends of the loop is

$$
V=-\frac{d \Phi}{d t}=-A \frac{d B}{d t}
$$

To know the limits on this approximation, a cubic variation for $\mathrm{B}(\mathrm{r})$ was assumed, $\mathrm{B}(\mathrm{r}) \approx$ $B_{O}\left(1+C\left(\frac{r}{r_{0}}\right)^{3}\right), B_{O}$ is the field at the center of the pulser coil, and $r_{O}=10 \mathrm{~cm}$ is the coil radius. $\mathrm{C}$ is a constant determined from the field near a straight line filamentary current. The magnetic field is constant inside the pick-up loop radius, $r_{p u}$, if $r_{p u} \ll 3 \mathrm{~cm}$.

One must also consider the frequency response of the pick up loop. The response time of such a loop is $\tau=\mathrm{L}_{\mathrm{pu}} / \mathrm{R}_{\mathrm{o}}$ where $\mathrm{L}_{\mathrm{pu}}$ is the inductance of the pick-up loop and $\mathrm{R}_{\mathrm{o}}$ is the terminating resistance of the loop, $50 \Omega . R_{0}$ is equal to the characteristic impedance of the co-ax cable used and the termination of the fast scope. The time $\tau$ should be less than any time scale in which significant change occurs.

As an example, a loop with a $5 \mathrm{~cm}^{2}$ area and width of $1 \mathrm{~cm}$ would have $L_{\mathrm{pu}}=29 \mathrm{nH}$ and a response time of $0.58 \mathrm{~ns}$, which is perfectly adecquate for this measurement. If the field rise time is 1.5 gauss/ns then the signal strength should be about 75 volts.

The voltage induced on the pick-up loop is equivalent to $\mathrm{Mdl}_{\mathrm{pc}} / \mathrm{dt}=(\mathrm{M} / \mathrm{L}) \mathrm{V}_{\mathrm{pc}}, \mathrm{M}$ being the mutual inductance between the pick-up and the pulser coil and $V_{p c}$ is the voltage applied around the pulser coil. Therefore, apart from a constant of proportionality, the applied voltage can be determined.

As was mentioned in section 5.1.2.3, the coil inductance was calculated from formulae to be $1 \mu \mathrm{H}$. An experimental measurement of the inductance yielded $2.9 \mu \mathrm{H}$. This measurement included extra inductance from the leads used to connect the segments, and from the choke feedthroughs. Hence, this is an upper limit on the Blumlein load. A lower limit on the field rise time is expected to be 1.3 gauss $/ \mathrm{ns}$ for a full $\pm 10 \mathrm{kV}$ charge on the Blumleins. 


\subsubsection{Results}

The set-up for the field rise time measurement was as follows. One of several pick-up loops that were made was positioned at the center of the pulser coil, axially and radially. The signal cable(s) to the scope were $50 \Omega$ impedance and greater than $50 \mathrm{~ns}$ propagation time in length. The signal was transmitted to a 7904 Tektronix $500 \mathrm{MHz}$ scope with $50 \Omega$ termination. Typically some attenuation was used in the signal line to reduce the signal magnitude for the scope. The scope was triggered by a pulse from a copper strip placed underneath the tack driver base. The strip coupled capacitively to the charged sheet. A signal goes out when the sheet discharges. This signal is a transient spike equal in magnitude to the charge voltage, and so it is attenuated before it is sent to the scope. The voltage signal from the pick-up loop was captured with a Polaroid camera using fast film.

Triggering the scope with the proper lead time was largely a matter of adjusting the cable lengths. While making these adjustments, as well as noise reduction adjustments, it was desirable to have a fast repetition rate. This was accomplished by not replacing the punctured insulating disc. The pulse generator was continuously being charged, and when the voltage reached a certain point, there was a spontaneous spark through the pre-punched hole and a pulse began. The breakdown regularly occurred at a charge of \pm 300 to 700 volts. The power supplies were usually set to $1 \mathrm{kV}$.

The first pick-up loop was made by wrapping some extra Blumlein foil around a cardboard core for the mylar strips. It was a single turn loop $1.8 \mathrm{in.}$ in radius and $5.5 \mathrm{in}$. long. Fig. 5.13.a contains the scope picture and 5.13.b shows the actual loop voltage and the magnetic field as a function of time derived from this. This shot was taken during a spontaneous discharge but the signal shape is typical. 


\section{a. Scope Trace of Pick-up Loop}

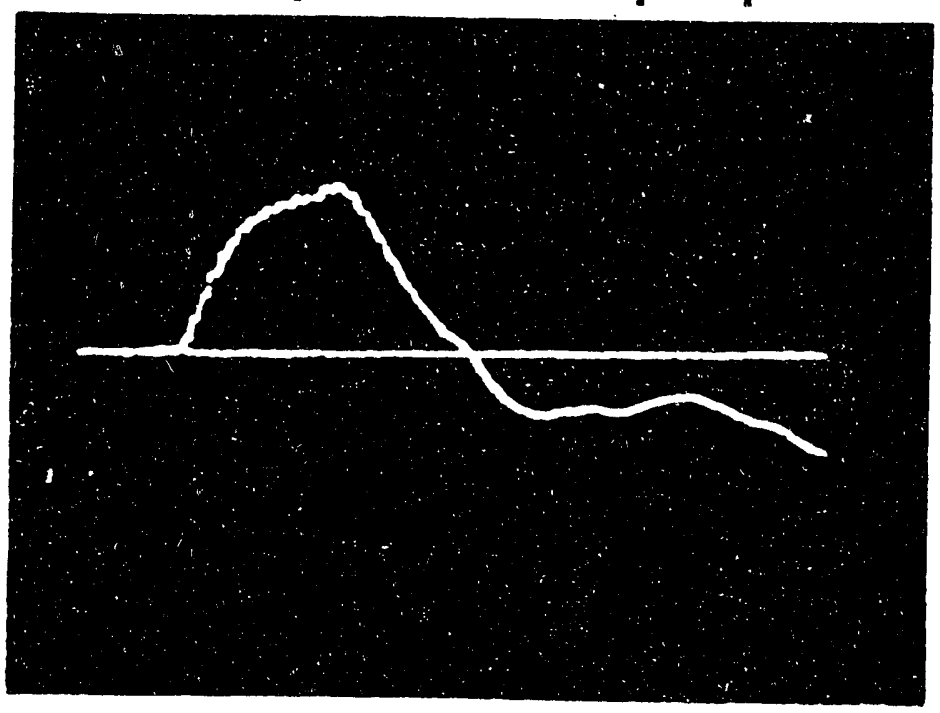

b. Pick-up Loop Signal and B(t)

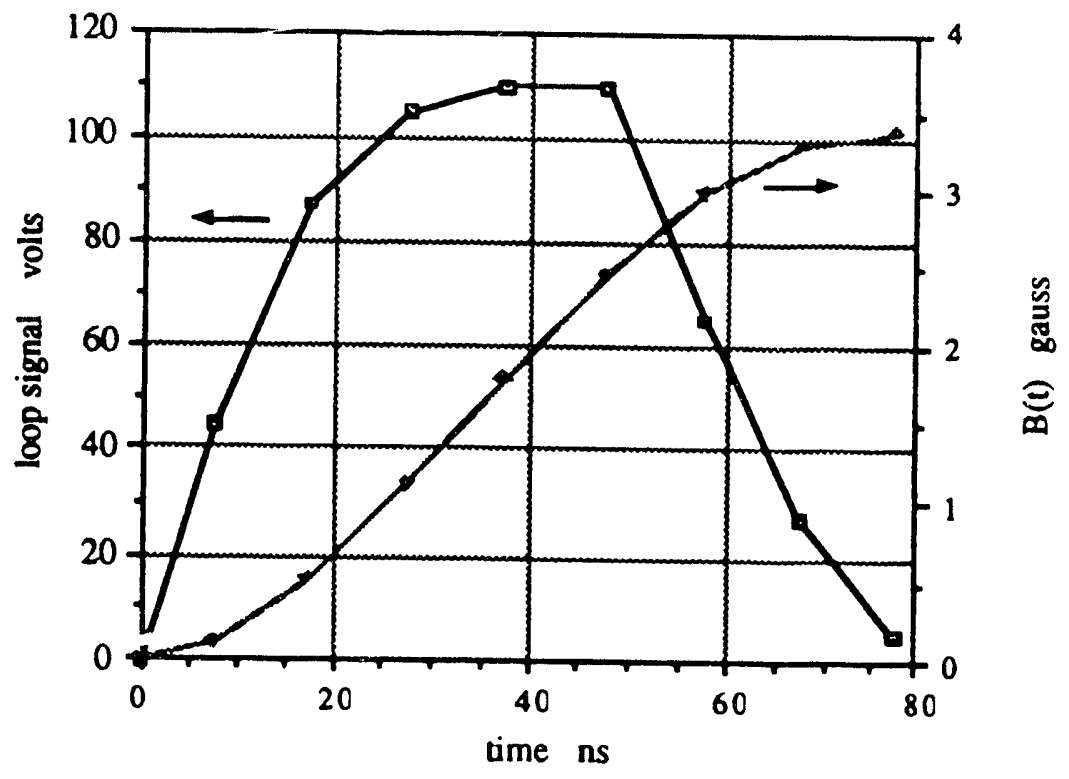

Fig. 5.13 a. The first scope picture of the first pick-up loop measuring the field rise time. The charge on the Blumlein lines was between 300 and $700 \mathrm{~V}$. b. This plot shows the measured loop signal and the field rise time calculated from it. 
One thing to note about this trace is that the voltage rises steeply for about $20 \mathrm{~ns}$ then continues to rise slowly until about $42 \mathrm{~ns}$ when it begins to drop. The voltage rise time is much slower than calculated from a simple model of a pulse propagating through a radial transmission line. However, the first switch made was not like that used in the simulation. In the first switch, the idea was to try to keep the switch impedance as constant as possible as a function of radius. So additional mylar sheets were added in between the copper sheets at larger radii. This may account for some of the slow rise time. Another factor that could result in a slower rise time might be local regions of high impedance in the lines, such as air gaps between the copper sheets. These areas tend to blunt a sharp pulse front. The accumulated effect of these high impedance regions could account for the observed voltage rise time. That the pulse lasts for $42 \mathrm{~ns}$ when it was designed for $32 \mathrm{~ns}$ is a bit puzzling but may be due to charge, trapped because of reflections, eventually discharging through the pulser coil.

In experimenting with loop parameters, one loop that was made attempted to reduce the noise by increasing the loop area to a $2.7 \mathrm{in}$. radius, with a $0.25 \mathrm{in}$. width. Thus, the signal magnitude was on the order of 700 volts for a $\pm 1 \mathrm{kV}$ charge. Another loop was made that was 1 in. $\left(\mathrm{r}_{\mathrm{pu}}=1.3 \mathrm{~cm}\right)$ in diameter and $0.5 \mathrm{in}$. in width. It was divided in half, and each half provided differential inputs to the scope. Typical signal levels were 10 volts for a $\pm 1 \mathrm{kV}$ charge. The measured response times for these pick-up loops were $21 \mathrm{~ns}$ for the large loop, $10 \mathrm{~ns}$ for the first cardboard core loop, and $1.2 \mathrm{~ns}$ for the small split area loop. Fig. 5.14 shows $B(t)$ derived from each of these $k$ iops for a pulse from a $\pm 1 \mathrm{kV}$ charge. The larger loop signals were corrected using a cubic variation for $\mathrm{B}(\mathrm{r})$. As expected, the smaller the loop the better it performed. The field rise time was 0.18 gauss $/ \mathrm{ns}$. This was determined from the split loop signal during the linear portion between 20 and $40 \mathrm{~ns}$.

Three more radial line switches were made after the first one. The second and fourth switches were essentially the same as shown in fig. 5.10. They both had the minimum two 
Fieid kise Times for the First Switch

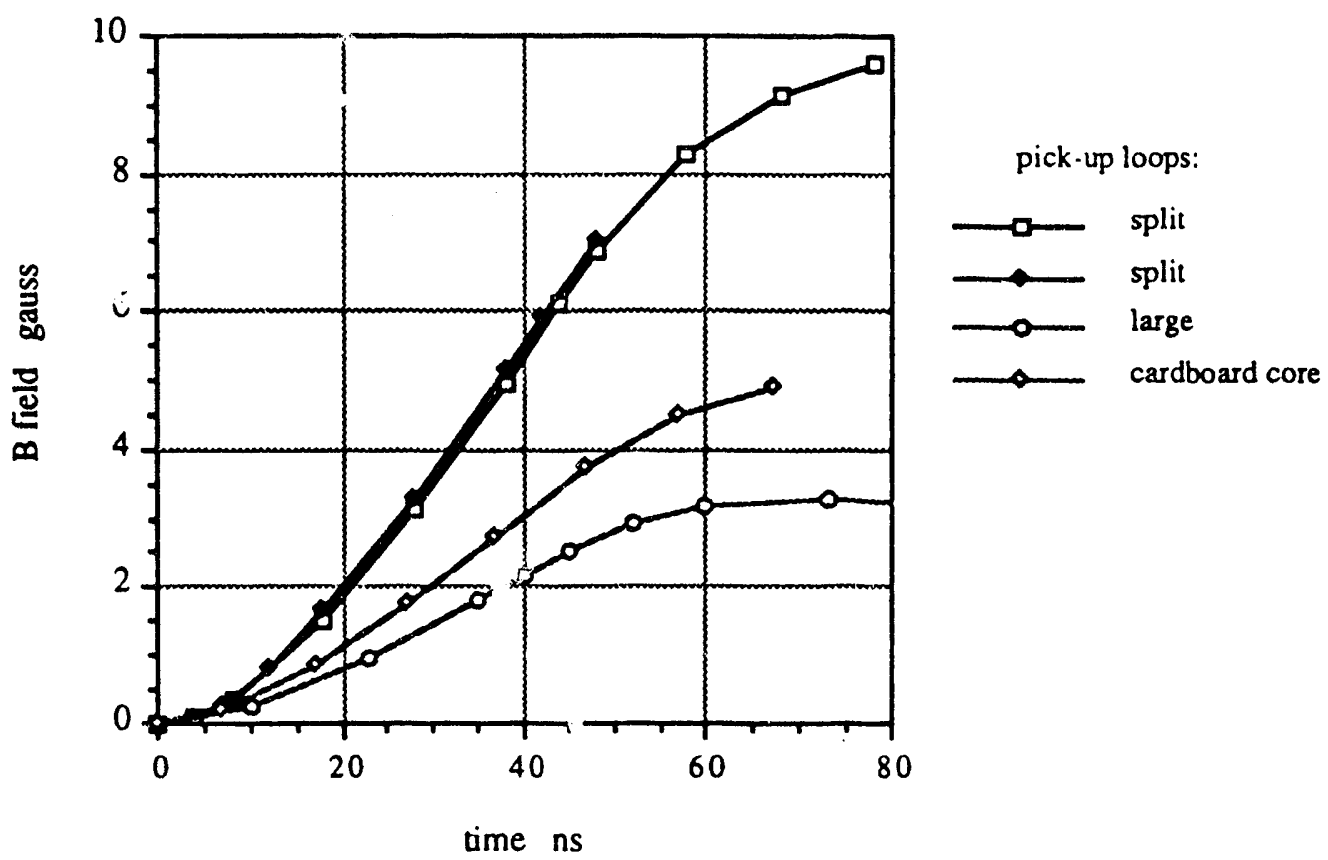

Fig. 5.14 Different loops were used to detect the rising magnetic field. The split loop pick-up has the best frequency response. All shots are done with a $\pm 1 \mathrm{kV}$ charge on the pulse generator. The best field rise time for the first ? witch is .18 gauss/ns.

sheets of $0.0075 \mathrm{~cm}$ mylar insulation between all the copper planes. The fourth radial line was made during the full charge tests. It was a redesign to prevent arcing under the replaceable disc.

The third radial line was a brief experiment and similar to fig. 5.10 but had a real copper ground plane that extended in to a $2.75 \mathrm{in}$. radius. Of course an additional two sheets of mylar were needed to insulate the ground plane.

After the first switch was replaced another even smaller pick-up loop was made. It was a thin strip of copper wrapped around a $3 / 16 \mathrm{in}$. $\left(\mathrm{r}_{\mathrm{pu}}=0.24 \mathrm{~cm}\right)$ diarn:ter insulating core. It was center grounded with two outputs. Its response time was not meisured but calculated to be $0.06 \mathrm{~ns}$. Its use was mixed with the split loop pick-up. Fig. 5.15.a shows the 
improvement from switch 1 to switch 3 using the split loop pick-up. Fig. 5.15.b shows the improvement from switch 3 to 2 and 4 using the little pick-up loop.

There is about a factor of two rise rate improvement in using the later switches. Fig.

5.15.b shows that the later switches are about the same in terms of rise rate, but the magnetic field begins to rise sooner in the second and fourth switches. Also noticeable is an increase of about 1.7 in the magnitude of the derived $B(t)$ function. The split loop gives a field rise of 0.38 gauss/ns and the little loop gives 0.63 gauss/ns. This demonstrates that the split loop pick-up is still not ideal. However, both loops were still used during the subsequent full charge tests.

In fig. 5.16.a, the little pick-up loop is used to compare a $1 \mathrm{kV}$ charge with a 4 and 5 $k V$ charge pulse. It is expected that $B(t)$ will scale linearly with the charge voltage so $B(t)$ is normalized by its charge to a $1 \mathrm{kV}$ pulse. This should yield an easy comparison. It seems that the increase in the magnetic field is less than expected. There is a $63 \%$ reduction in the rise rate per $\mathrm{kV}(0.40$ gauss/ns per $\mathrm{kV})$. Fig. 5.16.b gives the same sort of data using the split loop. $B(t)$ for charges of 3,7 and $10 \mathrm{kV}$ are shown. For the first $40 \mathrm{~ns} B(t)$ per $\mathrm{kV}$ is essentially the same, but there is still a decrease from the $1 \mathrm{kV}$ case. Similarly it is a $66 \%$ decrease $(0.25$ gauss/ns per $\mathrm{kV})$. Perhaps an expianation is that at higher charges the initial breakdown occurs sooner during the process of the tack piercing the insulation. It could be that the arc between the charge sheets is stretched over a greater distance so that there is a higher inductance in the switch.

In any event, if the split loop signal is used as an indication of the field rise, then it can be expected that there will be 150 gauss at the midplane by $70 \mathrm{~ns}$ after the start of the pulse, with a peak rise rate of about 2.5 gauss/ns when the full $\pm 10 \mathrm{kV}$ charge is used. This is greater than the expected minimum, but less than the 3.8 gauss/ns calculated in section 5.1.2.3. Hence, the measurement is in good agreement with our expectations. 

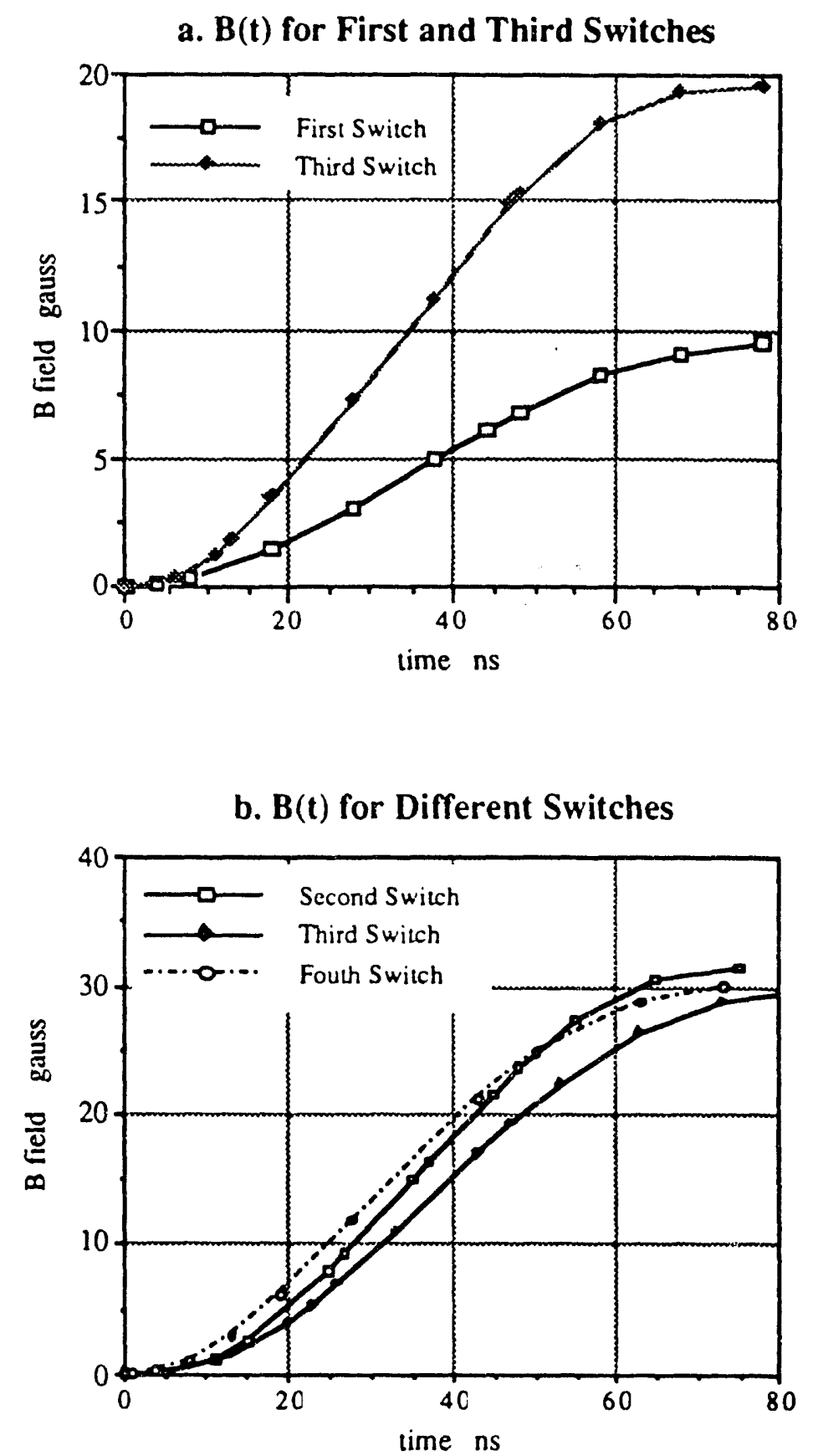

Fig. 5.15 $\mathrm{B}(\mathrm{t})$ for different radial switch line designs. The charge on the pulse generator is fixed at $\pm 1 \mathrm{kV}$. a. $\mathrm{B}(\mathrm{t})$ using a split pick-up loop. The third version is a big improvement over the first one. $\mathbf{b}$. $B(t)$ using a little pick-up loop. The second and fourth versions are a slight improvement over the third one. 
a. Normalized $\mathbf{B}(t)$ for Different Charges

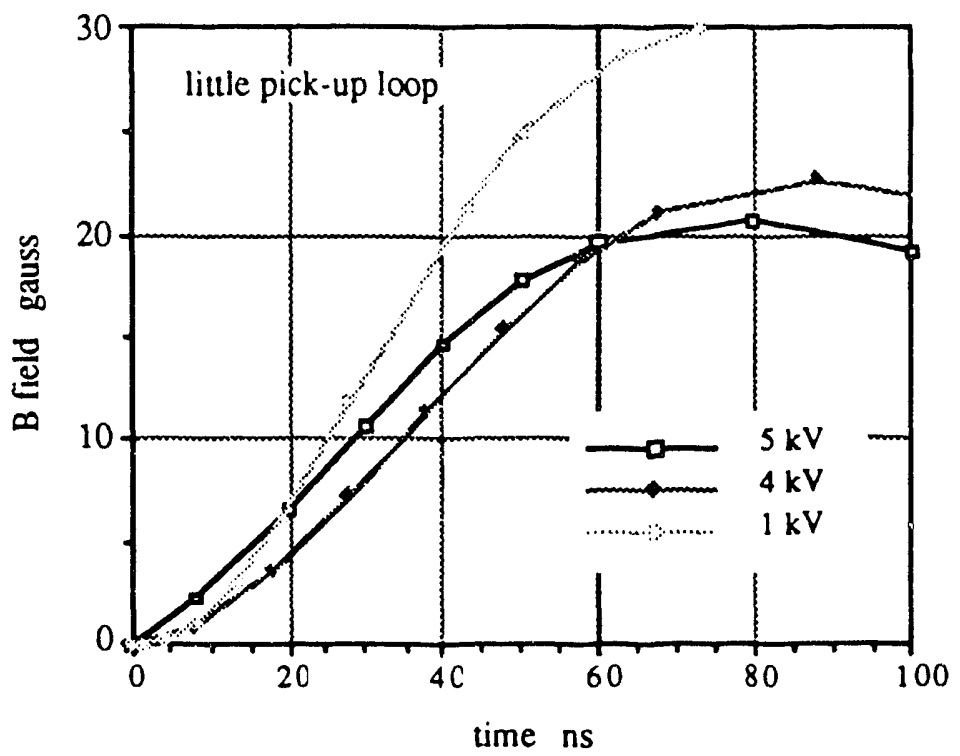

b. Normalized $B(t)$ for Different Charges

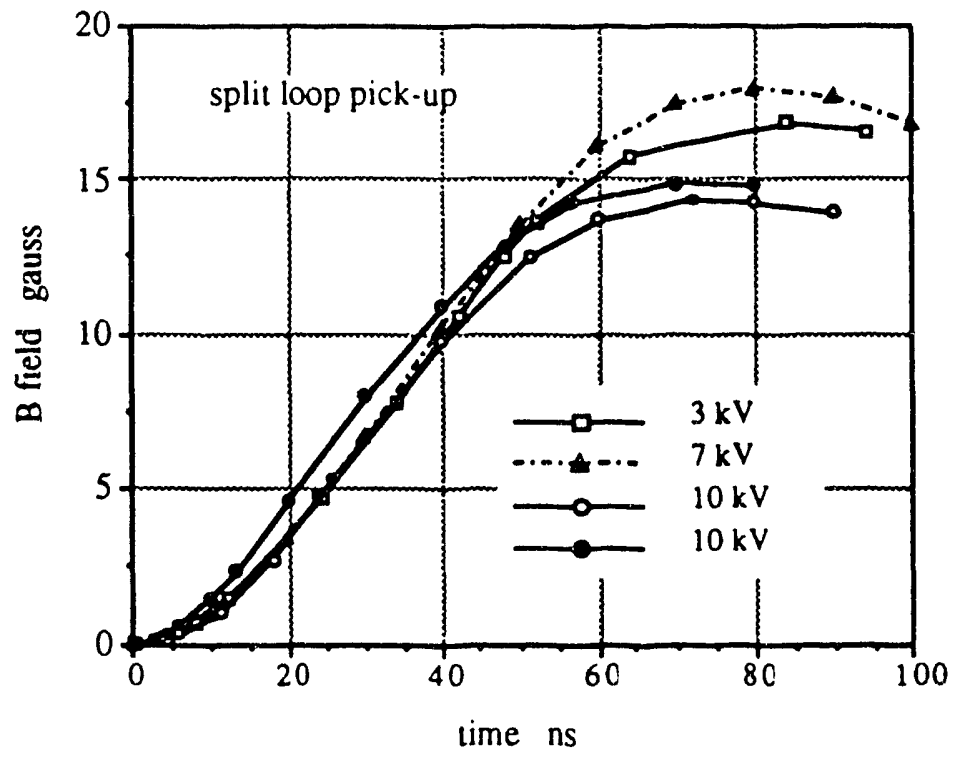

Fig. 5.16 $\mathrm{B}(\mathrm{t})$ is normalized to $1 \mathrm{kV}$ by dividing by the charge on the Blumlein lines. a. Shots using the little pick-up loop. For charges greater than $1 \mathrm{kV}$ the rate of field gain is less. b. Shots using the split loop pick-up. The field rise rates are linear functions of the charge. 


\subsection{Electron Beam Diagnostic}

The purpose of this electron beam diagnostic is to collect some information on the Mirrortron space potential. As the space potential rises, there will be an interaction with the beam that will slow down or accelerate the electrons. The bunching effects will cause an increase or decrease in the collected beam current. From the variation in current something about the potential can be said through comparisons with computer simulations.

At first, it was thought that an ion beam should be used because the energy of the beam that is cut off by the potential would correspond to the peak potential. However, the interaction is a little more complicated than that. An electron beam was used because it was simpler and easier to construct an electron source.

\subsubsection{Electron Gun}

The electron gun was designed to produce a few $\mathrm{mA}$ of beam current. Electrons were emitted from a hot wire and were accelerated by an electric field and launched through the center gas collimation tube down the axis of the main vacuum chamber. The beam then passed through the center $3 / 4$ in. hole and out of the plasma/cavity region. The beam was then collected at a detector.

Originally the gun contained two additional electrostatic plates behind the furst accelerating gap that were designed to focus the electron beam electrostatically. Because the gun was placed on axis in a strong magnetic field, the beam was strongly collimated by the magnetic field, and the extra two plates were not necessary. Visual inspection of the beam in a high density background gas near the detector indicated that the beam was approximately $0.5 \mathrm{~cm}$ in diameter. Fig. 5.17 shows the gun design and placement in the plenum chamber.

A power supply was designed to provide a high filament current to the gun. The filament was biased negative, up to $-10 \mathrm{kV}$ if necessary. By means of a variac the filament 
current could be controlled, up to 20 amps. Usually 15 amps was the threshold for electron emission from the wire. Up to 5 or $6 \mathrm{~mA}$ of current could be collected at the detector.

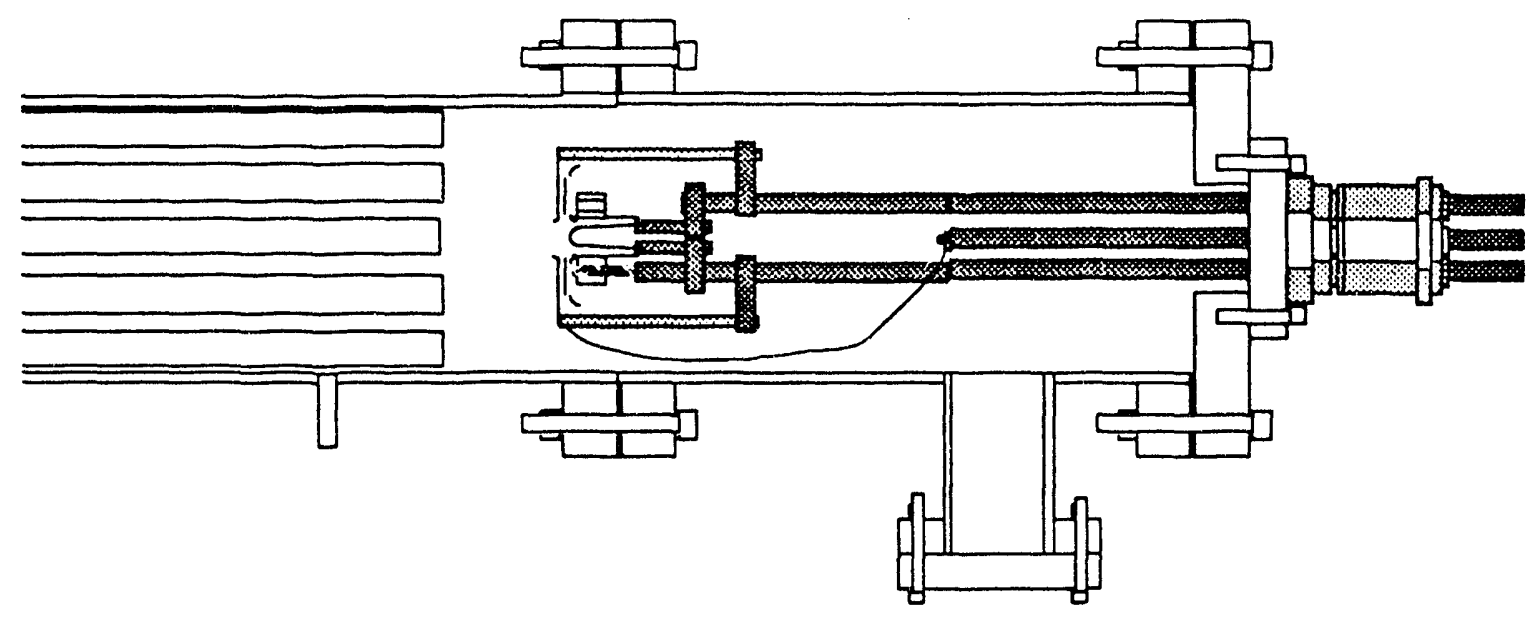

Fig. 5.17 The electron gun. The gun is mounted on the end of the plenum chamber. Electrons are emitted from a regatively biased hot filament and accelerated through the center of the gas collimation array.

\subsubsection{Beam Detector}

The beam detector consisted of a grounded cup that shielded two collection plates. The opening was covered by a screen mounted on the cup. The inside of the cup was then electrostatically shielded. Of the two plates inside, the smaller one was a $2.5 \mathrm{~cm}$ ( $1 \mathrm{in}$.) round disc mounted on the center line of the detector and Mirrortron. The second disc was $2.5 \mathrm{in}$. in diameter and located behind the first plate on axis. This arrangement helped to locate the beam and position and center the experiment with respect to the magnetic field.

Also located in the cup were two independently biasable screens for energy analysis. The idea was that one could be biased positive to keep out plasma ions while the second was varied negatively to collect different energy electrons, either from the plasma or beam.

The detector collecting plates were located $93 \mathrm{~cm}$ from the midplane. This distance allowed bunching effects to occur in the train of electrons as they traveled to the collector. The detector is shown in fig. 5.18. 


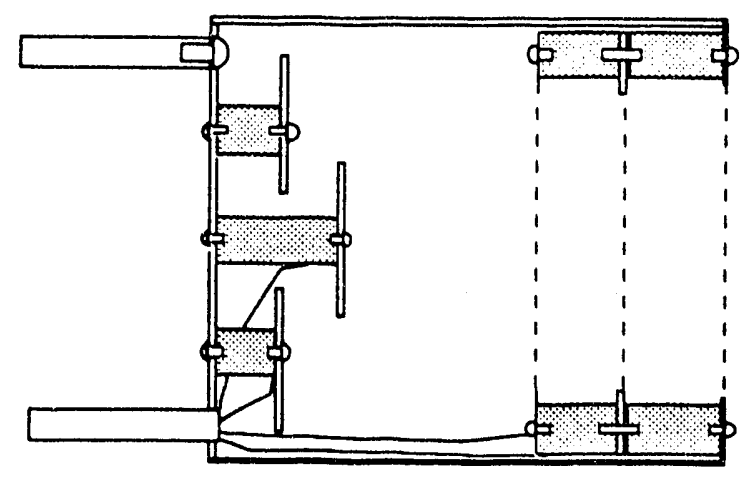

Fig. 5.18 The electron beam detector. There is a center and an outer collecting surface and two independent grids, all in a grounded shield cup.

\subsubsection{Interaction Modeling}

The best way to proceed in analyzing what can be gained from the electron beam diagnostic was to model the interaction by computer simulation. What I was looking for, ultimately, was a prediction of the electron current versus time. Hence, I began with an analysis of the current.

Current is just the amount of charge collected on a conducting surface per unit time. In a monoenergetic, steady-state, the beam density is constant (no bunching or rarefaction) and electrons arrive at regular intervals. During bunching electrons are collected faster and hence the current goes up. The velocity of the electrons does not affect the magnitude of the current increase, but merely where in the beam train bunching occurs, consequentlt, where in time the current increases. The question is then how to calculate the current given an array of times of arrival at a detector for a series of electrons passing through the potential region, and corresponding array containing the velocity of these electrons.

Once the beam is collected the current profile remains constant because now all the electrons travel at the same velocity: the drift velocity of electrons through metal. The current collected at the detector is

$$
I(t)=e n(t) v(t)
$$


where $n(t)$ is the electron density per unit length at the detector at time $t$ and $v(t)$ is the velocity of the local electrons at this time. For discrete positions and times, the time of arrival of the ith electron at the detector is $t_{i}$, while the $i+1$ electron is still a distance $x_{i+1}$ from the detector. The effective electron density is just the inverse of the electron spacing, i.e., $n_{i+1}=1 / \Delta x_{i+1}$. The velocity of the $i+1$ electron is

$$
v_{i+1}=\frac{\Delta x_{i+1}}{t_{i+1}-t_{i}}=\frac{\text { distance to detector from } x_{i+1}}{\text { time to travel to detector from } x_{i+1}}
$$

When electron $i+1$ strikes the collector, the current is

$$
\mathrm{I}_{\mathrm{i}+1}=\frac{\mathrm{e}}{\mathrm{t}_{\mathrm{i}+1}-\mathrm{t}_{\mathrm{i}}}
$$

Hence, to calculate the detector current we need only take a sequential difference of the time of arrival array and then invert it. The time of arrival array must be in chronological order. If not, it is a simple matter to sort the array.

The problem now becomes how to calculate a time of arrival array for a train of electrons. The equations to solve for an electron traveling through a varying space potential $\Phi(\mathbf{x}, \mathrm{t})$ are

$$
\begin{aligned}
\frac{d \mathcal{E}}{\mathrm{dt}} & =v \frac{\partial \phi}{\partial \mathrm{x}} \\
v & =\sqrt{\frac{2 \mathcal{E}}{\mathrm{m}}} \\
\frac{\mathrm{dx}}{\mathrm{dt}} & =v
\end{aligned}
$$

$\mathcal{E}$ is the kinetic energy of the electron, and $\phi=\mathrm{e} \Phi$ which is the potential energy of the electron. A second order differential scheme from these equations gives the following set of equations 


$$
\begin{aligned}
& \mathcal{E}(\mathrm{t}+\Delta \mathrm{t})=\mathcal{E}(\mathrm{t}-\Delta \mathrm{t})+2 \Delta \mathrm{t}(\mathrm{t}) \frac{\partial \phi}{\partial x}(v(t) \Delta t, t)+O\left(\Delta \mathrm{t}^{3}\right) \\
& v(\mathrm{t}+\Delta \mathrm{t})=\sqrt{\frac{2 \mathcal{2}(\mathrm{t}+\Delta \mathrm{t})}{\mathrm{m}}}+O\left(\Delta \mathrm{t}^{3}\right) \\
& x(\mathrm{t}+\Delta \mathrm{t})=x(\mathrm{t}-\Delta \mathrm{t})+2 \Delta \mathrm{t} v(\mathrm{t})+O\left(\Delta \mathrm{t}^{3}\right)
\end{aligned}
$$

The error terms are on the order of $\Delta t^{3}$.

The procedure for solving the problem then is to launch an electron at an initial time $T_{0}$ and then advance its position at intervals of $\Delta t$ until it reaches the detector at time $\mathrm{T}$. The time is then recorded in a time of arrival array. The next electron is then launched at a time $T_{0}+\delta t$. The procedure is repeated until the transient potential is over. The current is then calculated from this array as mentioned above. $\Delta t$ should be made small because it determines the accuracy of the equations. $\delta$ thas only to do with the resolution of the current and can be made as large as looks acceptable. $\Delta t$ should be much less than the time scale on which the potential changes, i.e., 30 ns. Trial values of $\Delta t$ of $0.1,0.05$, and 0.025 $\mathrm{ns}$ were used. They all produced the same result, so $0.1 \mathrm{~ns}$ was used for $\Delta \mathrm{t}$. Typically $1 \mathrm{~ns}$ was used for $\delta$ t.

To use equations 5.16 an expression for $\partial \phi / \partial x$ is needed. An analytical expression can be used in most cases. A gaussian approximation was sometimes used, in which case $\partial \phi / \partial x$ is simple. A more realistic expression for $\partial \phi / \partial x$ can be obtained combining eq. 2.23, eq. 5.3, and an analytic approximation (usually parabolic) for the static background magnetic field. This becomes a rather complicated expression so a difference method was used in the computer code.

The potential is a function of the normalized magnetic field, $\underline{B}(x, t)$

$$
\underline{B}(x, t)=\frac{B_{\text {stal }}(x)+B_{\text {pulse }}(x, t)}{B_{\text {stat }}(x)} .
$$


For the static magnetic field $\mathrm{B}_{\text {stat }}(\mathrm{x})$, a parabolic function is used to approximate the field in the vicinity of the midplane.

$$
B_{\text {stat }}(x)=B_{m p}\left(1+\left(R_{m}-1\right)\left(x / x_{1}\right)^{2}\right)
$$

The appropriate value for the midplane field is used for $B_{m p} . R_{m}$ is the mirror ratio and $x_{1}$ is the scale length, in this case, $68 \mathrm{~cm}$. For $B_{\text {pulse }}(x, t)$ eq. 5.3 is used, except that it is multiplied by a normalized, time dependent function derived from the bench tests. A fourth order polynomial approximates the function and has the following characteristics: the field rate of rise is initially zero, the field rise rate is maximum at $30 \mathrm{~ns}$, and the field peaks at 150 gauss at $70 \mathrm{~ns}$. This polynomial goes to zero at $125 \mathrm{~ns}$, and afterwards is set to zero.

When $\underline{B}(x, t)$ is used in equation 2.23 we can get an idea of the expected space potential as a function of time. Fig. 5.19 shows the potential at different times. Here a midplane field of $700 \mathrm{~g}$ and a mirror ratio of $1.5 \mathrm{is} \mathrm{used.} \mathrm{If} \mathrm{the} \mathrm{midplane} \mathrm{field} \mathrm{is} \mathrm{reduced} \mathrm{to}$ $600 \mathrm{~g}$ (resulting in an increased mirror ratio of 1.75) then the maximum value of $\mathrm{B}$ is increased, but the higher mirror ratio has a stronger influence and the peak value of $\frac{\mathrm{e} \Phi}{\mathrm{kT}_{\mathrm{e}}}$ decreases to 0.2 . Otherwise, the $\mathrm{x}$ and $\mathrm{t}$ dependence appears much the same.

When electrons are initially sent through a rising potential such as this, it is expected that they will first be accelerated. Then once they have crossed the midplane they will be decelerated. However, because the potential has grown, the deceleration will be greater than the initial acceleration, and there will be a net energy loss for these electrons. They will travel slower and space out leading to a decrease in current.

As the potential rises to a peak the potential variation will be slow, and there will be little net change in electron energy after passing through the midplane. The current will begin to increase. When the potential begins to decay, the reverse process will occur, and the electrons will gain energy. These electrons will travel faster and tend to bunch up with the proceeding ones. This will result in a current that is greater than the original current. 
The Theoretical Mirrortron Potential

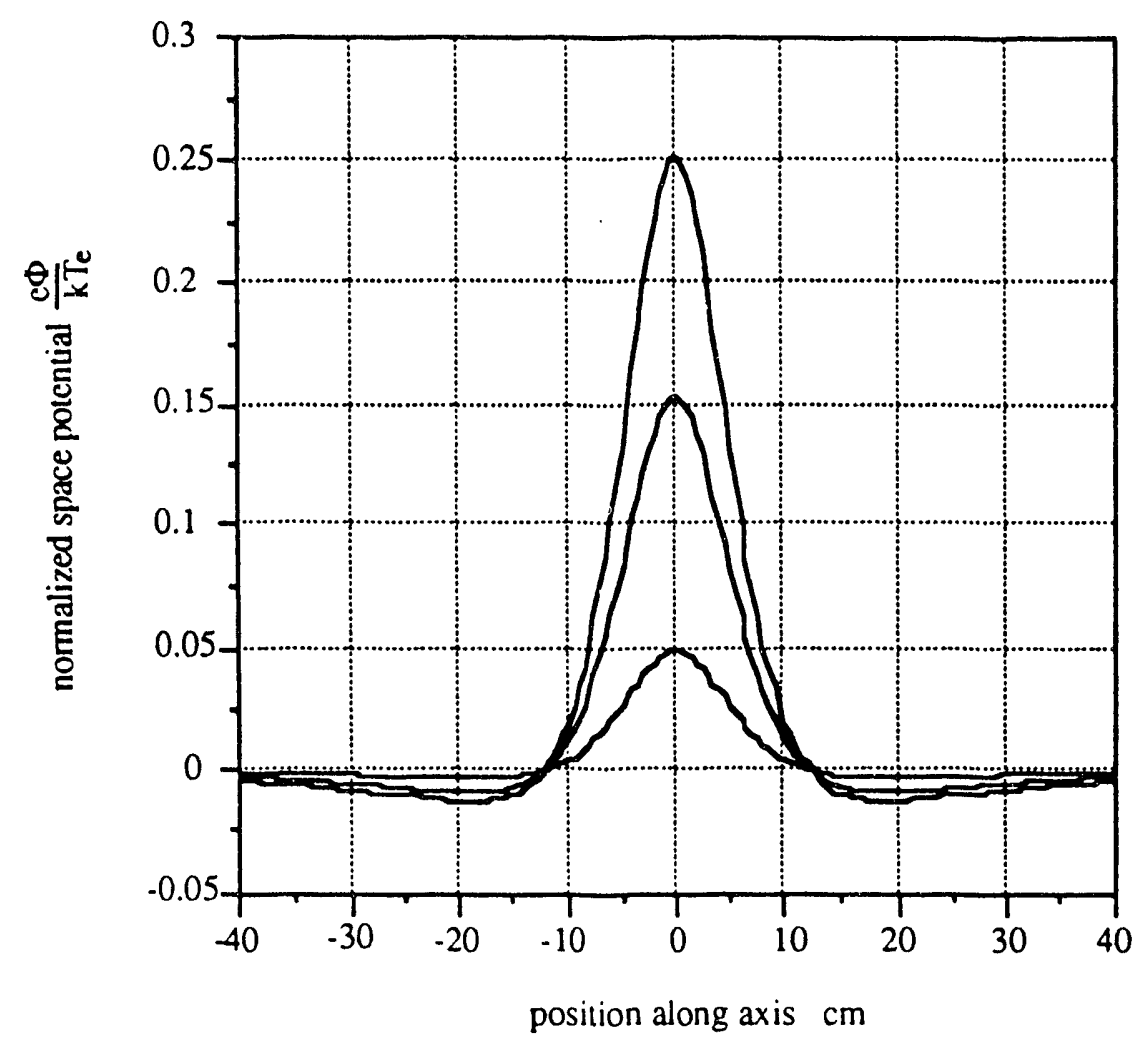

Fig. 5.19 This is the theoretically calculated Mirrortron potential at 20,40 and $70 \mathrm{~ns}$ after the start of the pulse. The potential is centered at the midplane where the field is 700 gauss with a mirror ratio of 1.5 . The peak field produced is 150 gauss. The effect of induced currents in the vacuum wall causes the potential to go negative beyond $12 \mathrm{~cm}$ from the midplane.

\subsubsection{Simulation Results}

Figs. 5.20-5.22 shows some results of this simulation. Electron energy and current at the detector are shown over time, and $t=0$ corresponds to the beginning of the pulse. There is a delay in the variation of the beam properties as the electrons travel to the detector. Each point represents an electron as detected at the collector. Fig. 5.20 shows the variation of an initial $500 \mathrm{eV}$ beam in a 100 and $10 \mathrm{keV}$ temperature plasma. In fig. 5.20.a and b many electrons were trapped or reflected because the potential energy change from the point 
of view of the electron was greater than 500 volts. This resulted in a complete cutoff of the current for about $50 \mathrm{~ns}$.

An array of electrons with more energy than they had initially is produced during the decay of the potential. They have caught up to each other and arrive at the same time at 150 ns. This results in a large current peak. From 150 to $180 \mathrm{~ns}$, the beam behavior becomes artificial as a result of abruptly setting the potential to zero at $125 \mathrm{~ns}$. Electrons after this time slow to their original velocity while the ones before them speed away resulting in another dip in current.

In the event that the potential is weaker because the plasma is not hot enough, or unable to screen out completely the ground potential of the vacuum wall, a simulation was done using $T_{e}=10 \mathrm{keV}$. The results are shown in fig. 5.20.c and $d$. The basic features are the same but less pronounced. Even in this case the variations are large enough that they can be detected easily.

The behavior generally agrees with what was expected except for the initial peak seen in 5.20.b. This is a result of the induced wall currents. The first electrons to arrive at the detector are the ones that are in the wings of the potential at its start. Here the potential becomes negative and repels the electrons out.

When beginning the electron beam tests, a 1 or $2 \mathrm{kV}$ charge on the pulse generator will be used. In this case a peak field of only 30 gauss will be produced. It will be of interest to see what kind of effect there will be on the electron beam. This effect is shown in fig. 5.21, again for two electron temperatures, 100 and $10 \mathrm{keV}$. The behavior is similar to that shown in fig. 5.20. Fig. 5.21.a appears to be an intermediate stage between fig.5.20.c and 5.20.a. Fig. 5.21.c is the beginning stage in which there is only a ripple on the beam energy and current . 
a. Electron Energy

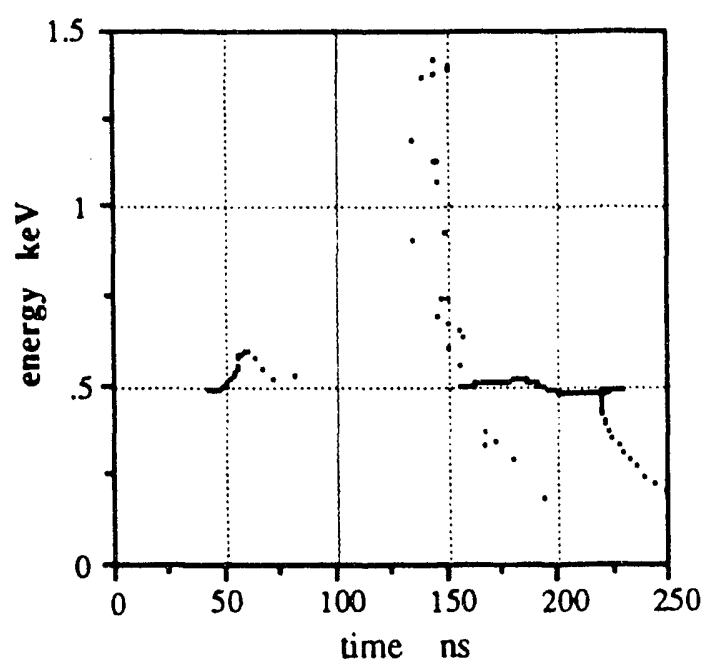

c. Electron Energy

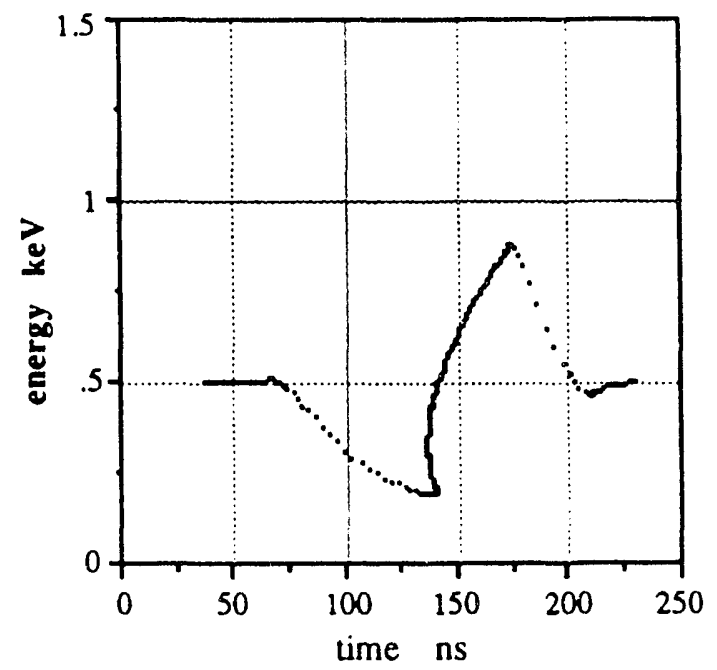

b. Electron Current

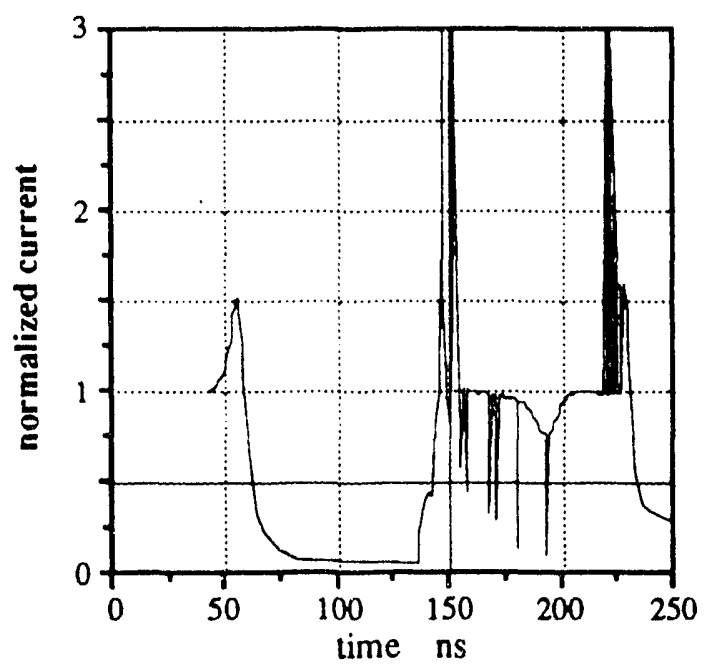

d. Electron Current

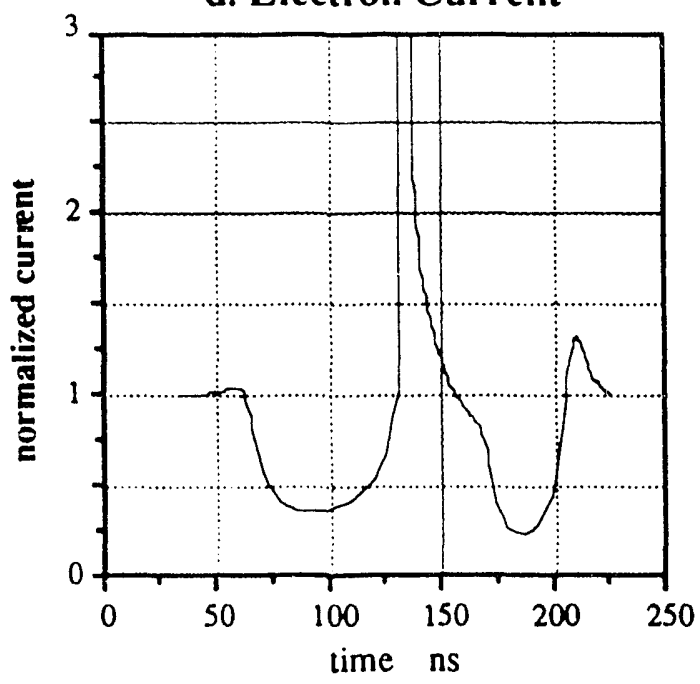

Fig. 5.20 Analysis of a $500 \mathrm{eV}$ electron beam passing through the Mirrortron potential. For $\mathbf{a}$. and $\mathbf{b}$. the beam passes through a potential formed in a $100 \mathrm{keV}$ electron plasma. For $\mathbf{c}$. and d. the potential is formed in a $10 \mathrm{keV}$ plasma. 

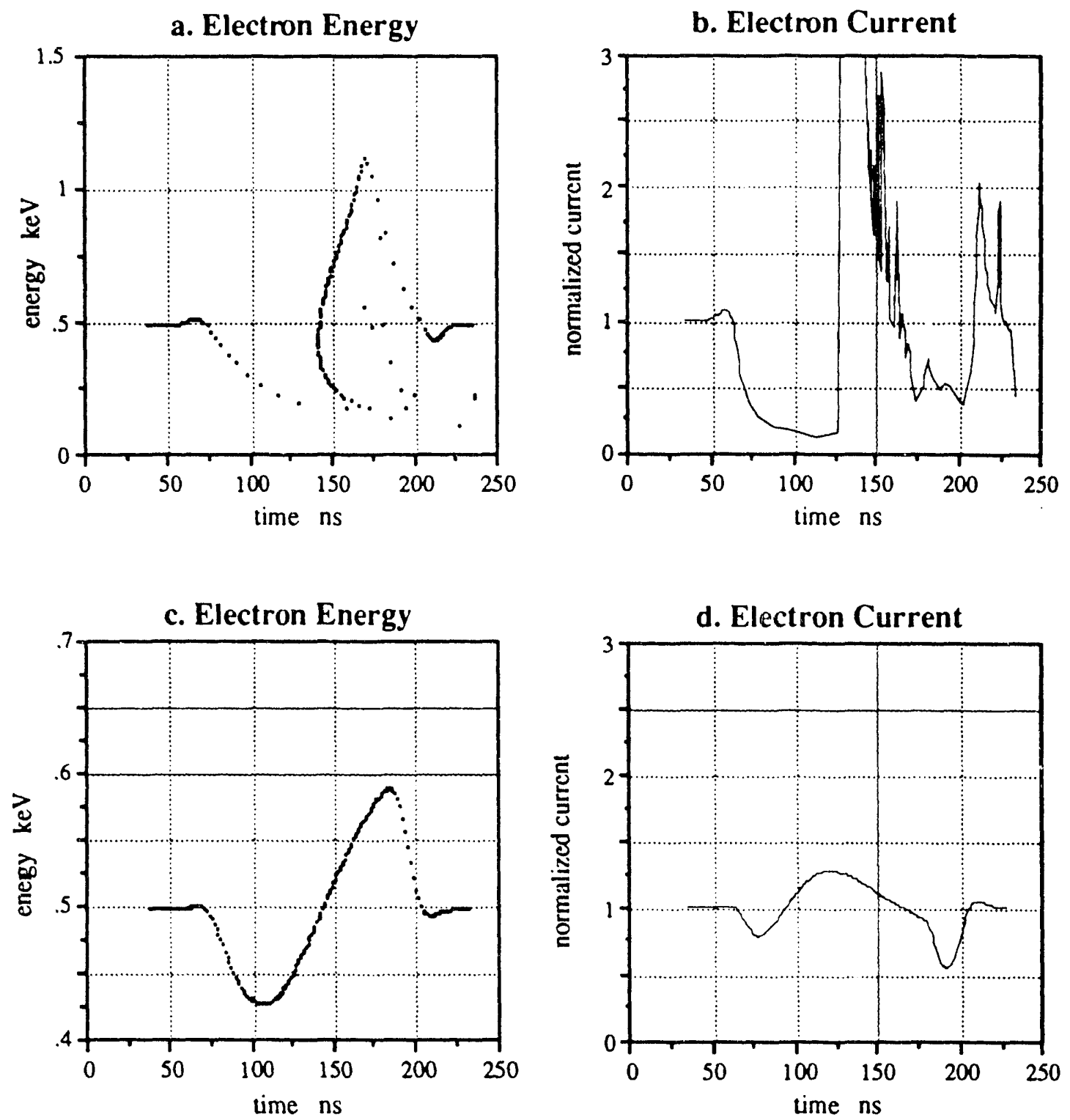

Fig. 5.21 Analysis of a $500 \mathrm{eV}$ beam passing through the Mirrortron potential. In this case the peak magnetic field is only 30 gauss. a. and $\mathbf{b}$. $100 \mathrm{keV}$ electron temperature plasma. c. and d. $10 \mathrm{keV}$ electron temperature plasma. 
The potential is linear with electron temperature and approximately linear with B (especially for smaller mirror ratios). The spatial behavior of the Mirrortron potential will not change as a function of $T_{e}$ and only slightly with $B$. Hence, the current variation approximately reduces to only a function of one parameter, the peak potential.

The peak potential in 5.21.c, 5.20.c, 5.21.a, and 5.20.a is $1 / 2,2.5,5$, and $25 \mathrm{kV}$ respectively. A direct rieasurement of the peak potential from the current is not possible. How zere, it can 'ie done by comparison with these simulations. Some help might come from energy analysis of the beam. By comparing the minimum energy of the electrons in fig. 5.21.c and 5.20.c, it appears that there is almost a factor of five in the energy decrease. This corresponds to the factor of five increase in the peak potential from $1 / 2$ to $2.5 \mathrm{kV}$. The potential could be determined by measuring the maximum energy decrease of the beam.

This can be done by varying the bias on the screens in front of the collector. When the bias screen is set at $450 \mathrm{~V}$, for example, there will be a sharp cutoff of the current when the energ: dips below $450 \mathrm{eV}$. Varying the bias on successive shots and looking for the cutoff will reveal the minimum energy point.

As the peak poteniual increases, the minimum energy electrons will arrive behind a front of more energetic electrons and become undetectable, as seen in fig. 5.21.a. If a more energetic electro ' beam is used, then the effect on the beam should not be as dramatic. In fig. 5.22 a $2 \mathrm{keV}$ electron beam is used. The result is that the electrons arrive in order but there is still enough of a variation in the current to be able to detect it.

From this computer simulation it looks as though the electron beam-potential interaction diagnı,stic will be effective as a means of determining the peak potential. In the next section this technique is applied to the experiment. Some significant current fluctuations were observed, but as a result of someihing unexpected. 

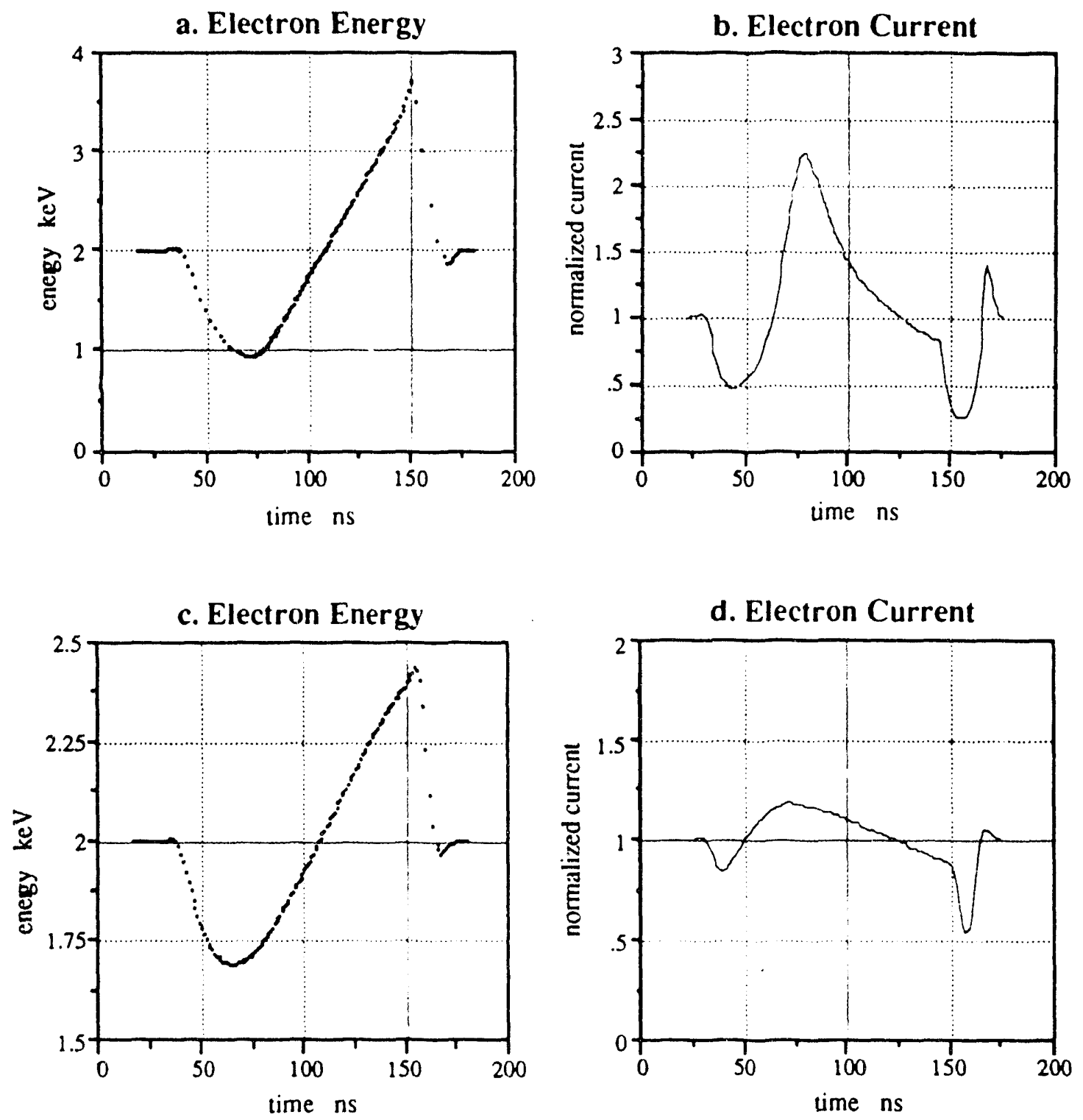

Fig. 5.22 Analysis of a $2 \mathrm{keV}$ beam passing through a $100 \mathrm{keV}$ electron plasma in the Mirrortron. $\mathbf{a}$. and $\mathbf{b}$. The peak magnetic field is 150 gauss. c. and $d$. The peak field is 30 gauss. 


\section{Chapter VI Results of Phase Two}

This phase consisted of plasma operation with the pulser coil installed and experiments using the electron beam. The first step that was taken was re-establishing the plasma operation of the Mirrortron. Equipment and instrumentation changes resulted in slightly different modes of operation. However, the plasma temperatures achieved were satisfactory. Experiments with the pulsed coil and the electron beam revealed some areas for improvement in the coil design. Extra electric fields associated with pulser coil feedthroughs permeated the plasma and affected the electron beam. However, this demonstrated the sensitivity of the electron beam. Also, these experiments indicated that the hot electron density after initial plasma decay may be too low.

The progress of the second phase can be divided into three parts. The first part was an attempt to reproduce the previous hot plasma results after the pulser coil was installed. In the second part, the $\mathrm{x}$-ray detector was moved back to its original position on axis from which it had been moved. In the third part, the electron gun and detector were installed and 
the second version of the Blumlein lines were connected to the pulser coil. In addition, the power supplies for the electromagnets that produce the mirror field were replaced.

\subsection{Machine Performance in Second Phase}

After the pulser coil was installed additional preparations were made to do experiments on the machine. The NaI x-ray detector was moved, and a plasma collection disc on a traveling arm was positioned behind the ventilation flange. The electron beam gun and detector cup were not installed during the first part of this experimental phase. However, the detector cup would eventually be in a place that would partially obstruct the $x$-ray flux to the $\mathrm{Nal}$ detector. Hence, the detector was then moved to a position closer to the vessel, but in an off-axis location.

The plasma collection disc was a spin-off of the electron source anode plate. The idea behind placing it on a linear motion feedthrough was two-fold. One purpose was to collect. escaping plasma along different flux lines. The other was to provide a movable target for the hot electrons, to be moved in and out of the line of sight of the $\mathrm{x}$-ray detector. Hence, there would be a means of calibration of the detector. In addition, the pulser coil was used to collect plasma current.

Part way through the second phase, the first power supply for the outer coils began to fail. Arc welder power supplies were found as replacements for both of the original supplies. These supplies had no trouble providing the full current necessary to produce the magnetic field called for by the design. Based on the current in the coils and on field calculations, the midplane field was determined to be 700 gauss, which is what the design originally called for.

\subsubsection{Comparison of X-rays Levels}

A new port was installed on the top of the extension vacuum section between the microwave cavity and the pump. The port was angled at $45^{\circ}$ and the line of sight through 
the port looked down toward the center of the ventilation flange. The $x$-ray intensity was expected to be higher at this closer position, hence, the signal would have been stronger. In addition, the angled position of the detector $\left(45^{\circ}\right.$ to match the centerline of the port) was expected to improve the magnetic shielding.

Despite the supposed advantages of the new position for the $x$-ray detector, the $x$-rays were very directional along the axis. Also, the photomultiplier was still in a region of high magnetic field which diminished its response. Hence, a smaller signal was detected. The difference was about a factor of a thousand. When the detector was in the on-axis position, typical $\mathrm{x}$-ray levels were up to $70 \mathrm{~K}$ counts/sec. When it was in the angled position on top the typical count rate was only a few hundred counts/sec.

In the detector's new position, the signal was small enough that the collection disc made no significant difference. The disc still had use as a particle collector. In the detector's old axis location, the $x$-ray intensity did not suffer measurably when the collector cup was installed. Even though the cup was in the axial line of sight, the field of view from the NaI position included the entire ventilation flange. And the energy of the $x$-rays that were being detected was large enough that the obstructions provided little attenuation. Therefore, the detector remained in that position.

The electron temperature for the hot component was measured to be about 30 to 60 $\mathrm{keV}$. This was satisfactory for the experiment. This later improved to $100 \mathrm{keV}$ when the new magnet supplies produced higher fields and when new microwave frequencies were used.

\subsubsection{Frequency Shift Measurement Problem}

Measurement of the frequency shift proved a bit more difficult for this phase of the experiment. The first problem was the response of the oscillator that was used to excite the resonant modes. A Microdot oscillator with a range of 500 to $1000 \mathrm{MHz}$ and maximum power of about 10 watts was used. Since the lowest cavity resonance was at $860 \mathrm{MHz}$, 
only the lowest resonances could be measured and only if their shifts were on the order of a few tens of $\mathrm{MHz}$. In the first phase of the experiment, it was noticed that there were gaps in the operating band (860-1000 MHz) where the oscillator would not operate. These bands did not occur when the output was into a matched dummy load. They were associated with the reflected power from the antenna and, perhaps, from a fraction of the high power heating frequencies that passed through the low pass filter. The first gap was from 870 to $879 \mathrm{MHz}$. The reflected power was reduced by placing isolators and attenuators in the coax cable. This was only partially successful. This was at the cost of reducing the input power to excite the shifted resonances. In the presence of a plasma the $Q$ factor of the cavity was reduced, and the resonances were harder to excite.

In the second phase, there were more, and wider band gaps. Isolators and attenuators did not seem to help. In the working bands it was not possible to find an excited resonance. This was due to modes being shifted into band gaps, or the decreased $Q$ factor from the pulser coil made the resonances undetectable. The density measured in phase one was assumed here while efforts to bring the Mirrortron into operating mode continued.

\subsubsection{Plasma Particle Collecting}

The movable tantalum disc and the pulser coil straps were used to collect plasma particles. The voltage on these particle collectors was a relative indicator of the plasma density. The floating potential of the disc was determined by connecting a digital voltmeter to the disc. The collected charge dissipated slowly through the meter's high internal impedance. It would almost always float negative at a few volts indicating that mostly electrons were escaping out the ends.

The pulser coil straps were almost always positive, indicating that the plasma ions were collected on the straps due to their larger gyroradius. The straps were grounded through $100 \Omega$ to prevent charge buildup. The measured voltage across this resistor indicated a few milliamps of current was collected. In certain instances during the electron 
beam experiments, the pulser coil collected negative current. This was perhaps due to an increased electron population that was trapped by the microwaves from the electron beam.

\subsubsection{Frequency Hunting}

In the first part of this phase, when the $x$-ray detector was counting 50 to 200 photons per second, $2355 \mathrm{MHz}$ was chosen for the fundamental (LF) heating power. This was the result of examining the 2300 to $2400 \mathrm{MHz}$ frequency range, and looking at the reflected power and the collected particle current. The second harmonic (HF) heating frequency was initially picked near 3860 because of strong coupling indicated here by resonance scans. Once the HF was applied, however, the plasma temperature was not very sensitive to frequency change. The typical electron temperatures were 30 to $40 \mathrm{keV}$. It was felt that it was necessary to run the magnetrons at a higher power than in phase one to make up for the decrease in the $Q$ factor, i.e., up to 100 watts. Sometimes the cables and antennae tended to overheat.

In the second part, after the $\mathrm{x}$-ray detector was moved to its original axial position, the count rate was immediately observed to increase to $60 \mathrm{~K}$ counts per second. This is about half of the maximum rate seen in phase one. An extended LF frequency search indicated that $2477 \mathrm{MHz}$ was optimized. The electron temperature was $35 \mathrm{keV}$ with LF power alone at this frequency, but improved to $55 \mathrm{keV}$ with $\mathrm{HF}$ power (at $3865 \mathrm{MHz}$ ). The forward power was 50 to 80 watts, which was perhaps higher than appropriate for comfortable continuous operation. At this point, we were satisfied that the presence of the pulser coil was not a serious detriment to plasma operation.

At this point, a break was taken in plasma operation to install the electron beam diagnostic and the Blumlein pulse generator. The electromagnet power supplies were replaced and the machine was now running with a smaller mirror ratio and a higher midplane field. 
During plasma operation in this third step, lower power was used to prevent the antennae from melting. With proper coupling it first appeared that little was sacrificed in terms of $\mathrm{x}$-ray output. The gas pressure was run around $210^{-6}$ torr. Another LF frequency $(2424 \mathrm{MHz})$ looked promising based on low reflected power and increased $\mathrm{x}$-ray flux.

There were some interesting curiosities noticed about the operation of the HF heating source. Once, after the plasma discharge had extinguished, I began to turn the HF power off. As I was turning it down, the glow discharge re-established itself. The magnetron output power was less than 10 watts at this point. Increasing the power from this point always led to a decrease in $\mathrm{x}$-ray flux until a higher power of about 50 watts was reached. Somehow improved coupling must have been achieved, apart from what can be obtained by tuning, because even at this low power state the $\mathrm{x}$-ray output did not seem very frequency dependent.

Also, for a time during these tests, it was not realized that the magnetic field was now operating at a high magnitude that did not allow second harmonic coupling to any microwave frequency less than $3920 \mathrm{MHz}$. Yet there was an observed interaction with the plasma that boosted the temperature of the hot component by 20 to $30 \mathrm{keV}$. Nevertheless, the $\mathrm{HF}$ frequency was shifted to a new resonance at $4008 \mathrm{MHz}$.

By this point repeatable plasma conditions were achieved with $\mathrm{x}$-ray fluxes of $55 \mathrm{~K}$ to $70 \mathrm{~K}$ per second and temperatures of 70 to $90 \mathrm{keV}$ with $\mathrm{H}_{2}$ and gas pressures of $1.610^{-6}$ to $2.010^{-6}$ torr using less than 10 watts of HF power at a plasma shifted frequency of 4015 $\mathrm{MHz}$ and about 50 to 60 watts of $\mathrm{LF}$ power at plasma-shifted frequencies up to $2430 \mathrm{MHz}$.

\subsection{Experiments with the Electron Beam}

The electron beam was first used as a method of centering the vacuum vessel with respect to the magnetic field. The electron gun and the collector cup were positioned on axis, but the beam followed the field lines. Initially, the vacuum vessel was so far off center that the bearn did not even pass through the center hole of the ventilation flange. The 
vessel position was adjusted. When the beam passed the ventilation flange, it entered the collector cup. The vessel was more finely centered by using the two concentric collecting surfaces at the back of the cup.

The first magnetic puise shots were calibration shots with no plasma. Their purpose was to see if the beam was deflected by the magnetic pulse, which would have implied that the beam was not centered with respect to the pulser coil. Also, the first shots were with less than the full charge on the transmission lines. It was felt that a full charge was unnecessary at first and would only increase chances for a breakdown within the line.

During these shots a fluctuation was noticed that appeared similar to what was expected when a plasma was present. Unfortunately, since this was a calibration shot there was no plasma, so this variation could not be due to plasma phenomenon. Fig. 6.1 shows the scope trace of the collected current.

The set-up for this measurement was exactly the same as if for a plasma experiment except that there was no microwave power sent into the cavity. Hence, there was no plasma during this shot except for any that might have been produced by the beam itself. The energy of the beam was $500 \mathrm{eV}$. There was a potential of $-100 \mathrm{~V}$ on the grid in front of the collector. The gas flow was the same as during plasma operation. The background pressure was $2.010^{-6}$ torr. During the steady-state gas flow the electron gun emission was adjusted so that $1 \mathrm{~mA}$ of current was collected. The shot began when the gas flow was stopped. Pumping continued for $1 \mathrm{sec}$, then the RF relay switches were activated. The microwave power would have been redirected into a dummy load had the sources been turned on. Approximately $100 \mathrm{~ms}$ later the tack switch was closed and the magnetic pulse began.

The charge on the Blumleins was $\pm 3 \mathrm{kV}$. This corresponds roughly to a 45 gauss peak field produced on top of the 700 gauss static field. From the trace it is observed that the steady-state current had increased to $1.8 \mathrm{~mA}$ due to the decrease in gas pressure before the pulse. The current decreased for 20 to $30 \mathrm{~ns}$, then began to flatten out for $50 \mathrm{~ns}$. This was followed by a sharp current peak, which lasted for $20 \mathrm{~ns}$. This behavior looks very similar 


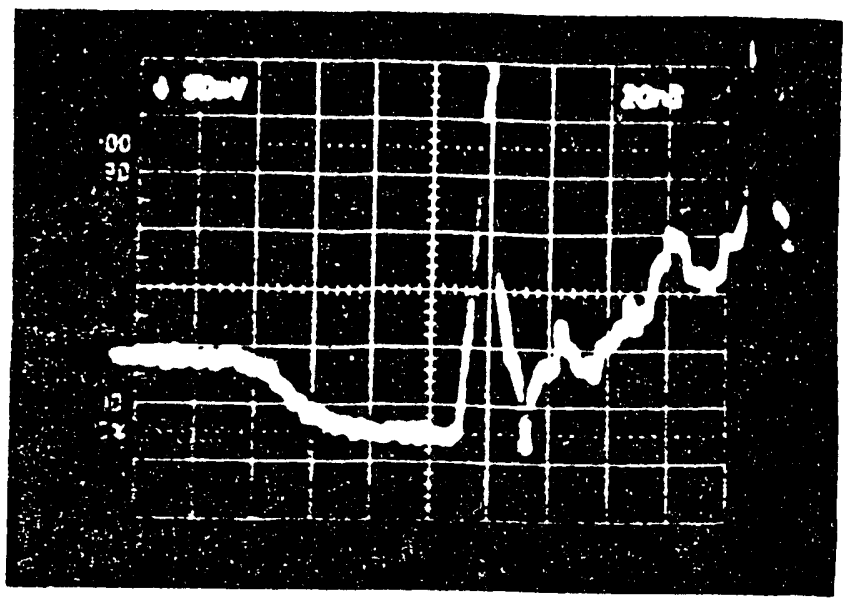

Fig. 6.1 A calibration shot showing fluctuations in the electron beam current during a magnetic pulse. The scale is $50 \mathrm{mV}$ per division into a $50 \Omega$ input impedance. This corresponds to $1 \mathrm{~mA}$ per division. The baseline is one division above the bottom. The time scale is $20 \mathrm{~ns}$ per division.

to that described in fig. 5.20.d or 5.21.b, both of which simulate a $500 \mathrm{eV}$ electron beam in a plasma. In both cases the time from the knee of the current decrease to the peak was about $80 \mathrm{~ns}$, just as in the measured current fluctuation above.

For a direct comparison with plasma operation, the same procedure was used but with the microwave power on. A steady-state plasma was created that produced an x-ray flux of $66 \mathrm{~K}$ counts per sec. on the detector. This is typical of a $70 \mathrm{keV}$ hot component plasma. The detector was set to count in $10 \mathrm{~ms}$ intervals. Once the gas was shut off the $\mathrm{x}$-ray level increased by about $10 \%$. Once the microwaves were stopped the $x$-ray level dropped by $90 \%$. The remaining $x$-ray flux decayed to zero over a period of $500 \mathrm{~ms}$. The tack switch closed $100 \mathrm{~ms}$ after the microwave shut-off. The results are shown in fig. 6.2.

The only significant difference occurs $100 \mathrm{~ns}$ after the fluctuation begins. However, this is not an important part of the pulse for comparison purposes. This is because the ions have moved enough by this point to make the plasma behavior unpredictable. The steadystate current before the pulse begins is about $1.7 \mathrm{~mA}$, a slight difference with the calibration shot and probably due to the plasma. The fact that there is little difference between the two 


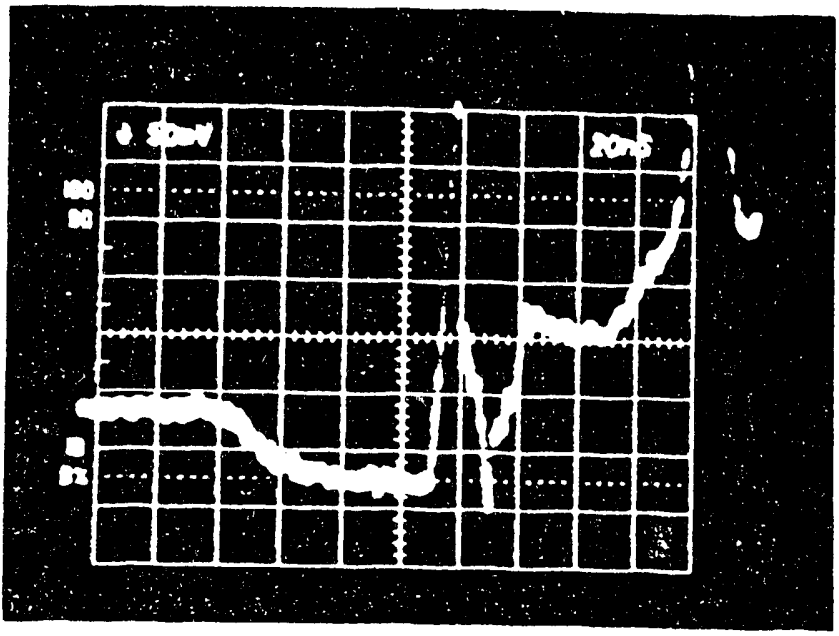

Fig. 6.2 The current fluctuation of an electron beam passing through a plasma during a magnetic pulse. The time and vertical scales are the same as in fig. 6.1.

shots indicates a low density problem. However, the cause of the current variation in vacuum must first be determined and eliminated if possible.

Further characterization was done to help determine the nature of the fluctuation. First, the beam energy was increased to see what effect there was on the detected current signal. A $1.5 \mathrm{~mA}$ current beam was used without a plasma or any feed gas. $A \pm 4 \mathrm{kV}$ charge pulse was used. Beam energies from 1 to $5 \mathrm{keV}$ were used. The result is shown in fig. 6.3. The initial dip and the following spike were decreased in magnitude as the energy increased. This confirms the idea that more energetic electrons have a weaker interaction with the potential pulse. What is interesting to note is that the broader current peak behind the initial current variation is unaffected. This suggests that this peak is not associated with the beam directly, but its source is probably from plasma produced by beam ionization.

Studies were done without the electron beam but with a plasma. A steady-state plasma, continually fed by gas and microwaves, as well as a plasma in decay mode were analyzed. What was revealed was that plasma particles were pushed out and formed a broad current peak at the same location in time as in the electron beam shots. These shots are shown in fig. 6.4. 
When a plasma was maintained in steady-state and without any bias on the grid of the detector cup, an electron current was collected; $0.4 \mathrm{~mA}$ on the center disc and $1 \mathrm{~mA}$ on the outer ring plate. When $-100 \mathrm{~V}$ was applied to the grid all plasma current was stopped. This was the operating mode of the cup when the shots in fig. 6.4 were taken. The electron temperature was $70 \mathrm{keV}$ with a gas pressure of $210^{-6}$ torr. The magnetic pulse was produced using a Blumlein charge of $\pm 4 \mathrm{kV}$. The microwave heating and gas feed were on during the magr.etic pulse in figs. 6.4.a-c. The gas and microwaves were stopped together about $40 \mathrm{~ms}$ before the tack switch closed in fig. 6.4.d,e. For these shots a Blumlein charge of $\pm 3 \mathrm{kV}$ was used.

In fig. 6.4.a the first current peak that is shown in the trace occurs at $100 \mathrm{~ns}$ on the time scale. The subsequent current rise begins at $140 \mathrm{~ns}$ on the scale and continues for over $100 \mathrm{~ns}$. These are also features in the beam current fluctuation when there is no plasma. An energy composition of the particle pulse is roughly determined by increasing the bias on the screen in front of the collector plates. Fig. 6.4.b and c show two of these shots. The first peak is determined to consist of electrons greater than $600 \mathrm{eV}$ but less that $1100 \mathrm{eV}$. The broad rise of the second peak is stopped by 600 volts but four or five constituent peaks still come through. The most energetic of the peaks in the second group is near the beginning of the group at $160 \mathrm{~ns}$, and is more than $1600 \mathrm{eV}$ and still not disappearing by 2100 volts.

Fig. 6.4.d is a shot taken with a decaying plasma. This current trace has the same features as fig. 6.4.a; there is an initial current rise then a bigger and broader one following. Of course the peaks are about half the size and not as wide, as would be expected from a smaller density plasma. The triggering has been adjusted so that the trace appears translated to the right on the scope scale, but the relative timing of the peaks is the same. In this case the current is virtually stopped when the screen voltage is increased to -600 volts. This is true even when higher charges on the Blumleins are used. 


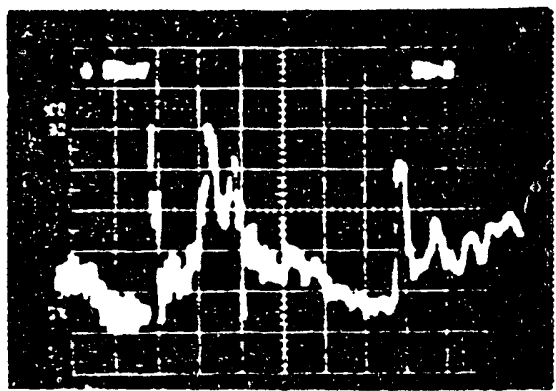

a. $1 \mathrm{keV}$

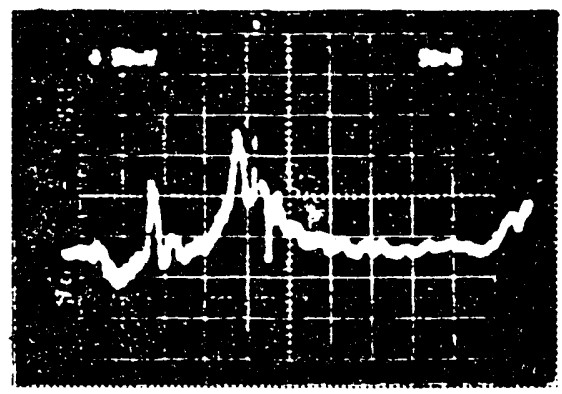

c. $3 \mathrm{keV}$

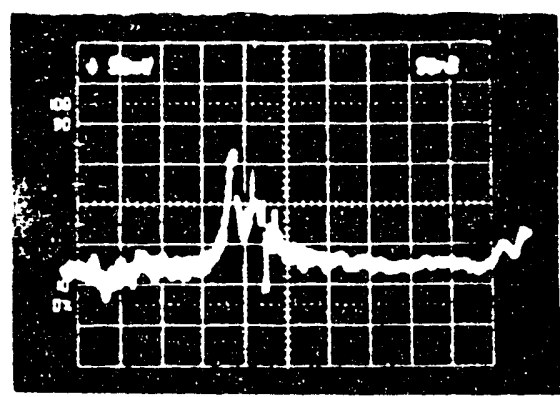

e. $5 \mathrm{keV}$

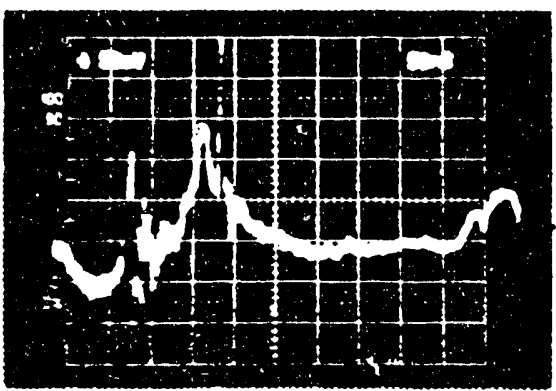

b. $2 \mathrm{kev}$

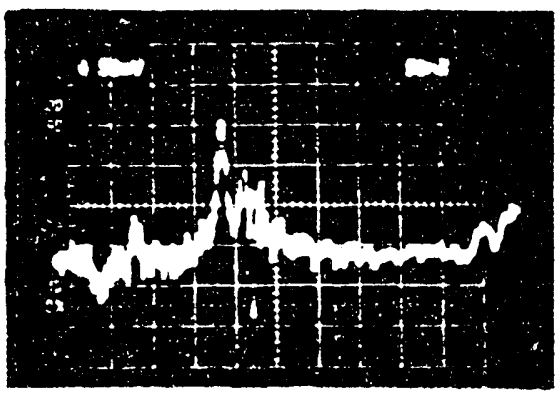

d. $4 \mathrm{keV}$

Fig. 6.3 Variation in the current fluctuation as a function of the electron beam energy. The vertical scale is $1 \mathrm{~mA}$ per division and the time scale is 50 ns per division. In c.-e. an additional $20 \mathrm{~ns}$ of lead time was added to the trace so that they appear shifted to the right compared to $\mathbf{a}$. and $\mathbf{b}$. 


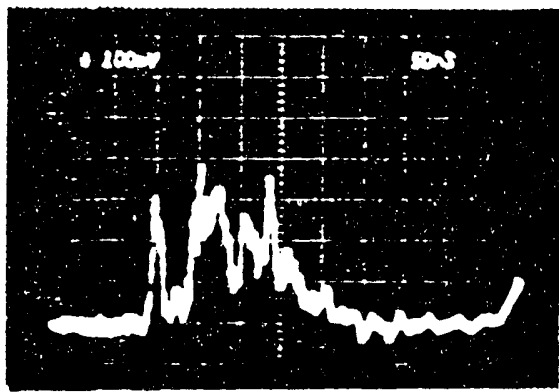

a. screen: $-100 \mathrm{~V}$

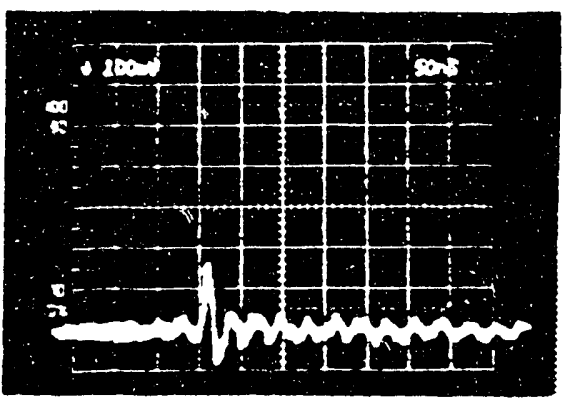

c. screen: $-1100 \mathrm{~V}$

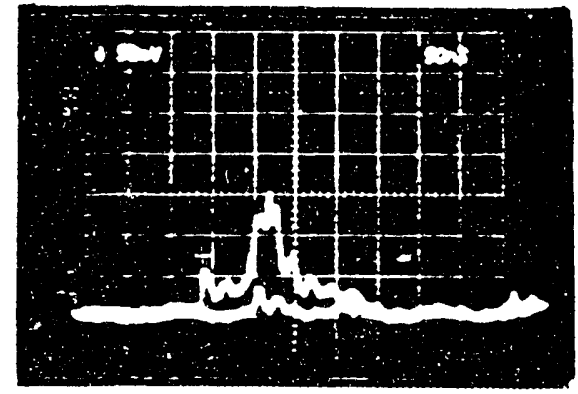

d. screen: $-100 \mathrm{~V}$

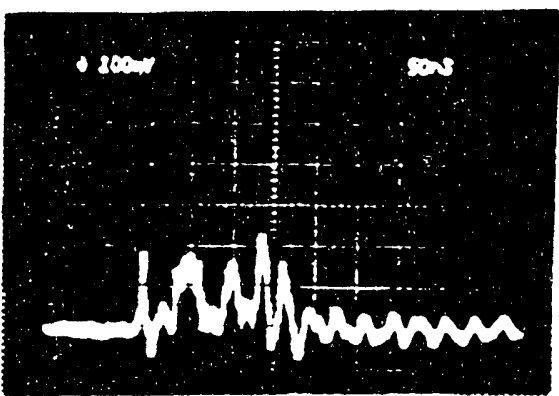

b. screen: $-600 \mathrm{~V}$

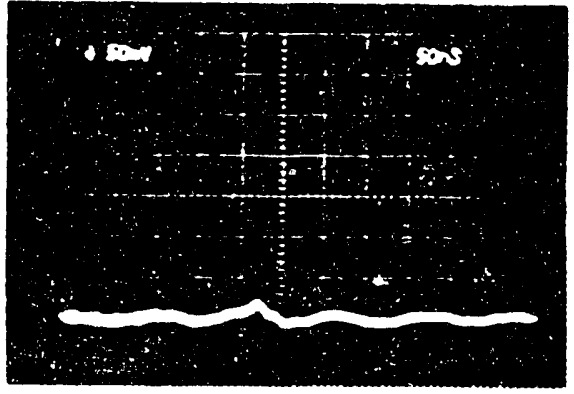

e. screen: $-600 \mathrm{~V}$

Fig. 6.4 The effect of the magnetic pulse on the mirror trapped plasma. Here there is no electron beam; only plasma current kicked out by the magnetic pulse is collected. In a.-c. the plasma is maintained continuously by gas and microwaves during the pulse. Various biases are used on the screen in front of the detector cup. Vertical scale is $2 \mathrm{~mA}$ per division. In $\mathbf{d}$. and $\mathbf{e}$. the microwaves and gas have just been turned off and the plasma is decaying. Vertical scale is $1 \mathrm{~mA}$ per division. All pictures are $50 \mathrm{~ns}$ per division. 
It is clear that after abous $150 \mathrm{~ns}$ plasma accelerated by the pulse floods into the detector and obscures the beam signal. Hence, the important part of the signal is the first $150 \mathrm{~ns}$. The first peak in the plasma-only and the first peak in the beam shots appear to occur at the same time after the pulse begins. It is not clear whether the electrons in this bunch are beam electrons or from the plasma (produced by either the beam or RF).

To identify the electrons in this bunch, an energy analysis of the electron beam in a vacuum was done. Fig. 6.5 shows the total beam current and current with energy components griater than 600,1100 , and $1600 \mathrm{eV}$. The pulse is produced with $\pm 4 \mathrm{kV}$ charge on the Blumlein generators. The first n.mow peak and the second broad peak are still the dominant features of the traces.

The microwave plasma and the plasma formed by beam ion; . ation are similar in that they both produce a peak relatively late in the pulse. The question still remains about how to explain these peaks as well as the first initial dip in beam current. One possibility might have something to do with the increased magnetic field. Magnetic compression could have increased the current density. A test of this possibilty was done where the midplane magnetic field was increased to the mirror field strength. The current variation in this case was unchanged. This also shows that this current fluctuation effect does not involve the mirror trapped portion of the beam-plasma, if any.

If this effect is some kind of interaction with the plasma associated with the beam itself, then the beam-plasma density will be an important quantity. The density of the beam electrons will be $610^{6} \mathrm{~cm}^{-3}$ if the current is $1 \mathrm{~mA}, 500 \mathrm{eV}$ energy and $0.5 \mathrm{~cm}$ diameter. The density of the associated plasma could be sornewhat highicr based on the ionization rate, of the beam. To check this idea, the background gas pressure was increased to raise the associated plasma density. The electron gun emission was reduced to lower th: density. In the first case a 500 e. $\mathrm{V}$ beam in a base pressure of $310^{-7}$ torr was changed when the $\mathrm{H}_{2}$ gas pressure increased to $2.010^{-6}$ torr. The only effects were that the steady-state current level 


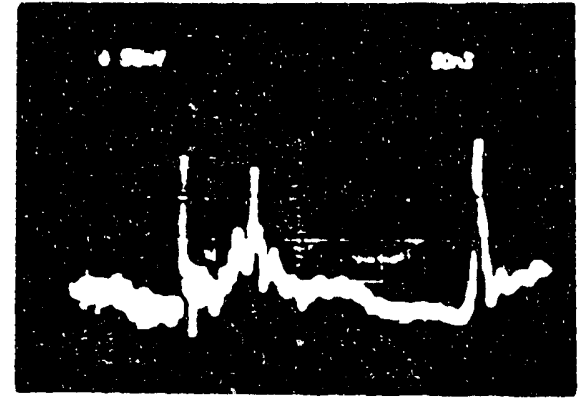

a. screen: $0 \mathrm{~V}$

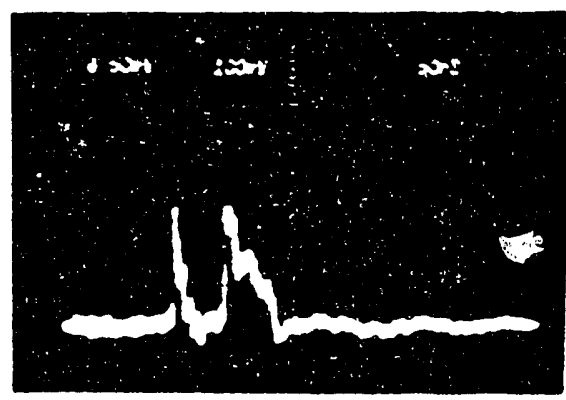

c. screen: $-1100 \mathrm{~V}$

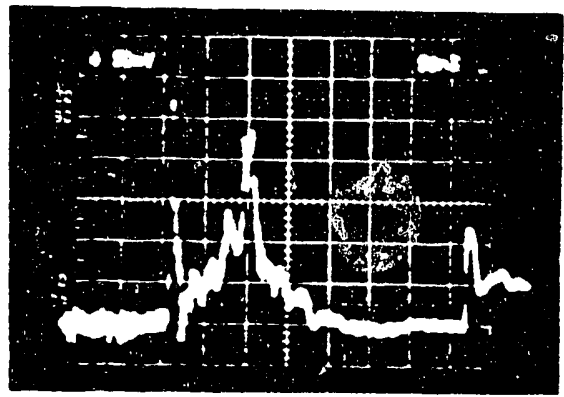

b. screen: $-600 \mathrm{~V}$

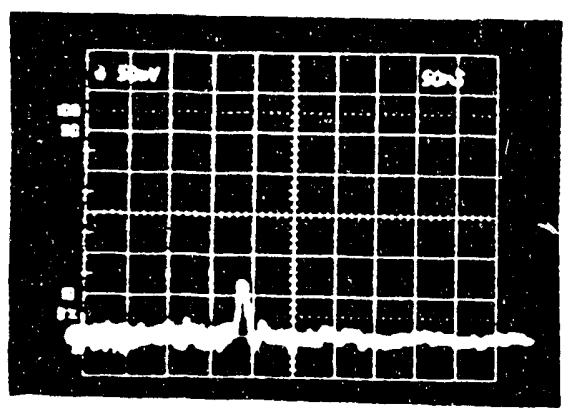

d. screen: $-1600 \mathrm{~V}$

Fig. 6.5 Energy analysis of a $500 \mathrm{eV}$ electron beam in a magnetic pulse. The eleciron beam is in a vacuum. The scales are $1 \mathrm{~mA}$ per division and 50 ns per division.

was decreased from $5.5 \mathrm{~mA}$ to $3 \mathrm{~mA}$, and she second broad peak had additional current peaks as in fig. 6...a and $b$.

In the second case the beam current was reduced to bareiy detectable levels. The results are shown in fig. 6.6. The magnitude of the current peaks are reduced in proportion to the initial current level. The only interesting change is that the first current peak is not as energetic $(<1100 \mathrm{eV})$ as is shown in fig. 6.5.c. Clearly it seems that by this point the beam electrons are not responding to the collective effects of a plasma, but rather, they are responding to externally applied forces.

Eventually it was thought that there might be some electrostatic fields associated with the pulser coil thai was interacting with the beam. In ihis case the description "electrostatic" means that the potential in the space around the pulser coil is determined by a solution to 


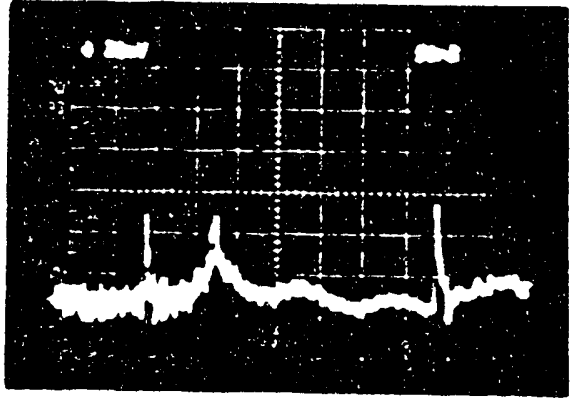

a. screen: $0 \mathrm{~V}$

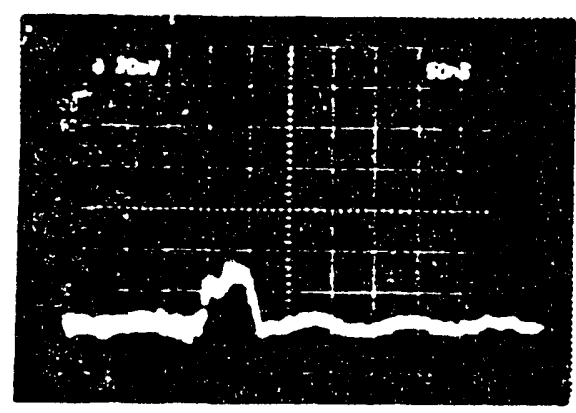

c. screen: $-1100 \mathrm{~V}$

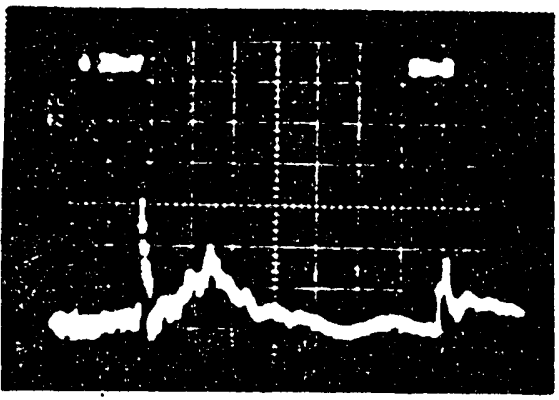

b. screen: $-600 \mathrm{~V}$

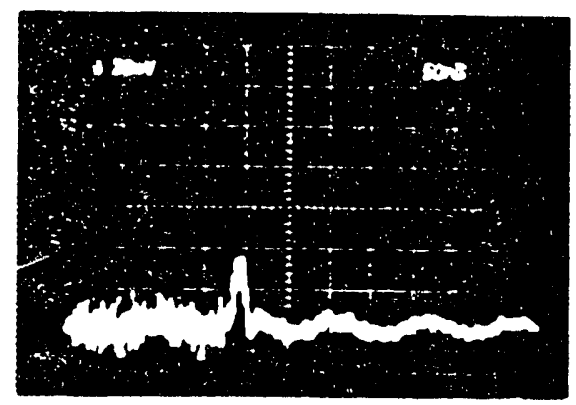

d. screen: $-1600 \mathrm{~V}$

Fig. 6.6 Energy analysis of a $500 \mathrm{eV}$ electron beam in a magnetic pulse. The electron beam is in a vacuum. The beam density is much reduced; the current is about .1 $\mathrm{mA}$ The scales are $.4 \mathrm{~mA}$ per division and $50 \mathrm{~ns}$ per division.

Poisson's equation. Boundary conditions are specified by the potential on the pulser coil. This description is justified because the characteristic time for change in the coil current is small compared to the time it takes to radiate the solution throughout the space. Each of the eight strap segments have one end attached to a feedthrough $3.8 \mathrm{~cm}$ (1.5 in.) downstream of the beam, and the other end is $3.8 \mathrm{~cm}$ upstream of the beam. The feedthroughs downstream are attached to the Blumlein generators that provide a positive voltage. Similarly the upstream feedthroughs are attached to a negative potential. Essentially an electrostatic field is formed across the midplane of the vessel. It is strongest near the wall but will nave a non-zero value on axis. This postulated potential is depicted schematically in fig. 6.7 . 


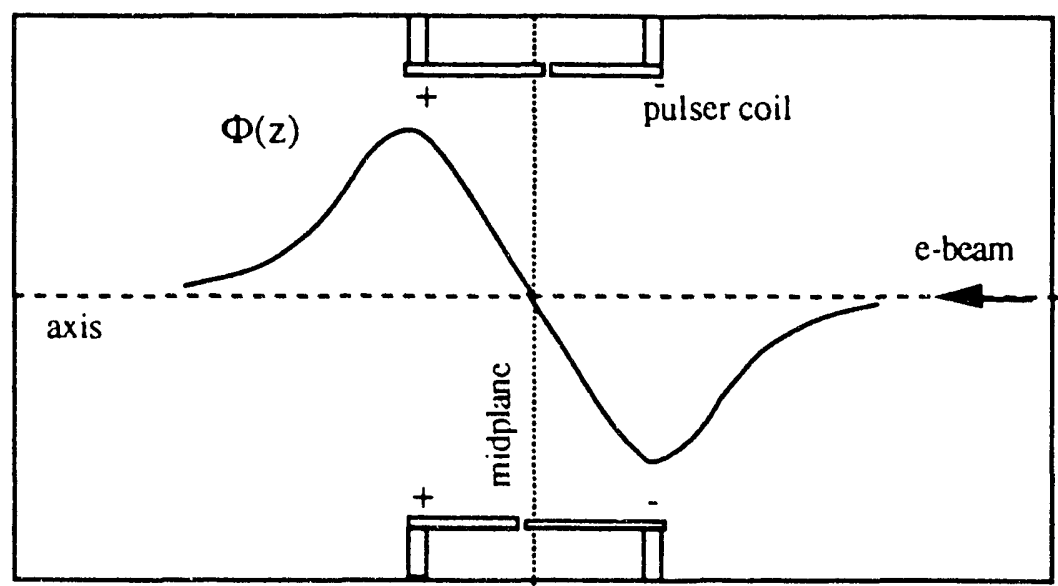

Fig. 6.7 A rough dipiction of what the electrostatic potential of the pulser coil might look like on axis

Because this potential is not symmetric with respect to the midplane, this hypothesis could be easily tested by reversing the polarity on the Blumlein generator. The background mirror field would also have to be reversed so that the pulsed field will still cause a compression of the electron beam as opposed to an expansion. This effect is then eliminated from this test. One advantage of the new arc welder supplies is that they have a polarity switch which makes this transition simple. Fig. 6.8 shows the result of this test. In this shot the beam energy is $500 \mathrm{eV}$ and the Blumlein charge is $\pm 4 \mathrm{kV}$. Here there is a change in the current waveform unlike any other that was produced after variations in the operating parameters. This clearly points to the problem. 


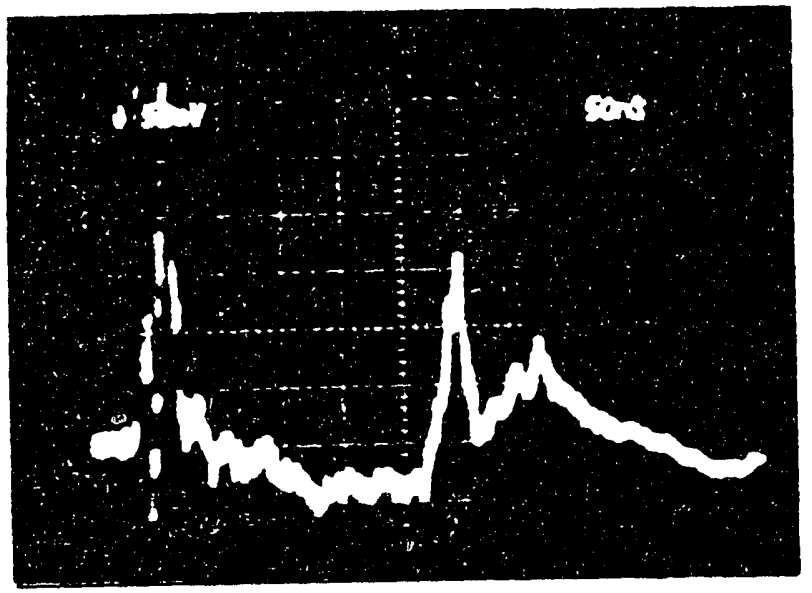

Fig. 6.8 Result of reversing the Blumlein generator polarity on the electron beam current. The vertical scale is $1 \mathrm{~mA}$ per division and the time scale is $50 \mathrm{~ns}$ per division. There is no plasma.

\subsection{Modeling the Electrostatic Field}

A variety of mathematical functions were used to simulate the potential sketched in fig. 6.7. One of the first ones used is the expression in 6.2

$$
\frac{\Phi_{0}(z / L)}{\left(\frac{(z / L)^{2}+2}{3}\right)^{3 / 2}}
$$

Eq. 6.2 is based on the form of a magnetic field produced by a current ring. In this case the potential is maximum at $\Phi_{0}$ when $z=L$. The function is shown in fig. 6.9. Also shown is a piecewise continuous model which will be discussed later.

$\Phi(z)$ is a time varying potential and will be multiplied by a function $T(t)$ that has a maximum value of one. $T(t)$ is expected to behave similar to the voltage that is applied to the feedthroughs. Fig. 6.10 is a picture of the voltage applied to the feedthrough terminal 


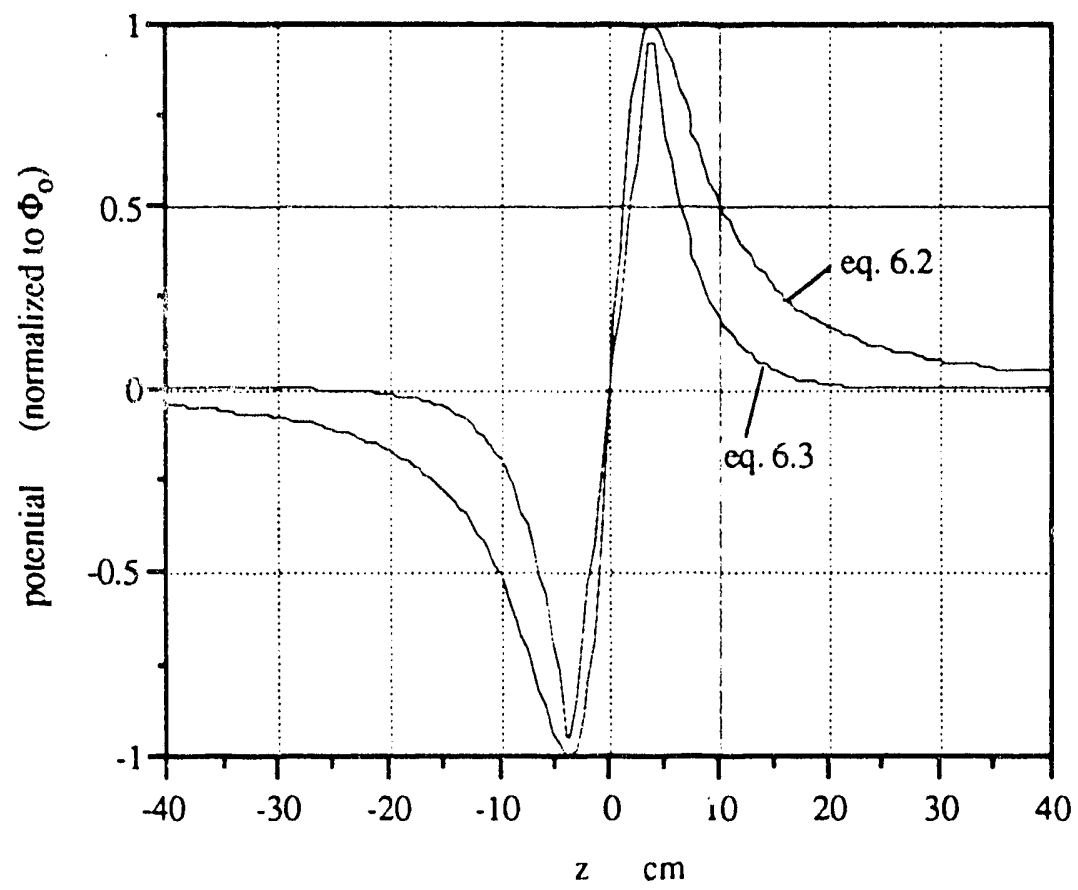

Fig. 6.9 Mathematical functions used to model the pulser coil electrostatic potential. Here the functions are plotted for $\mathrm{L}=3.8 \mathrm{~cm}$, the distance of the feedthroughs from the midplane.

from a Blumlein generator. The line was charged to $4 \mathrm{kV}$ and produced a $4 \mathrm{kV}$ pulse. A spline fit of two polynomials is used to describe the voltage form; a fourth order polynomial to describe the positive portion of the curve between 0 and $57 \mathrm{~ns}$, and a parabola to describe the negative portion of the curve between 57 and $125 \mathrm{~ns}$. The potential is set to zero after $125 \mathrm{~ns}$.

In fig. $6.11 \Phi_{0}$ is set to 500 volts and $\mathrm{L}=3.8 \mathrm{~cm}$. Also, a $500 \mathrm{eV}$ beam is used. Fig. 6.11.b and d show what kind of current fluctuation might be expected for forward and reversed polarity on the pulser coil. Comparing these plots with figs. 6.5.a and 6.7 we can immediately see the similarities. In the case of normal polarity there is a drop in the current followed by a large current spike. When the polarity is reversed, a current peak comes first followed by a drop in current. 


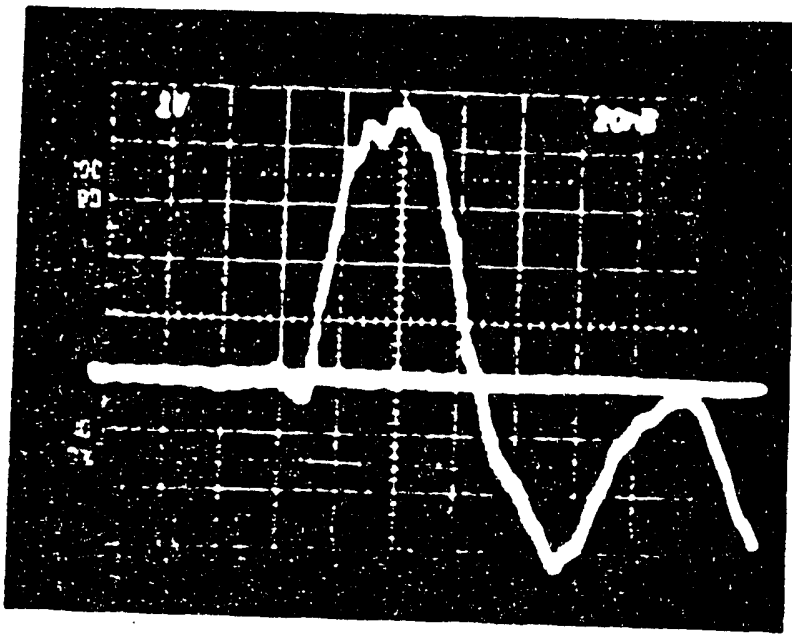

Fig. 6.10 Scope picture of the pulse generator signal applied at one external feedthrough to the pulser coil. The Blumlein was charged to $4 \mathrm{kV}$. The signal is measured using $-60 \mathrm{~dB}$ of attenuation. The vertical scale is then $1 \mathrm{kV}$ per division. The time scale is $20 \mathrm{~ns}$ per division.

There are differences too. The timing in the simulations is compressed compared to what is observed. In the actual current fluctuation the time from the knee of the current drop to the spike is about $80 \mathrm{~ns}$. In this simulation it is about $35 \mathrm{~ns}$. In the reverse polarity case the current depression after the first spike lasts about $200 \mathrm{~ns}$, but in the simulation it is only $60 \mathrm{~ns}$. It is a bit harder to make a comparison in this case because the current front that appears at $120 \mathrm{~ns}$ in fig. 6.11.d is due to behavior late in the pulse which is not modeled as accurately as the early stages.

Fig. 6.12 shows results for a weak potential on-axis. The variation in the energy of the arriving electrons is shown with a very minimal skew, i.e., the waveform is not as tilted om upper left to lower right. This results in smaller bunching effects and smaller current f ctuations. An increase in $\Phi_{0}$ will increase the magnitude of the energy variation and hence increase the skew or tilt of the electron train as seen in the energy diagrams. 

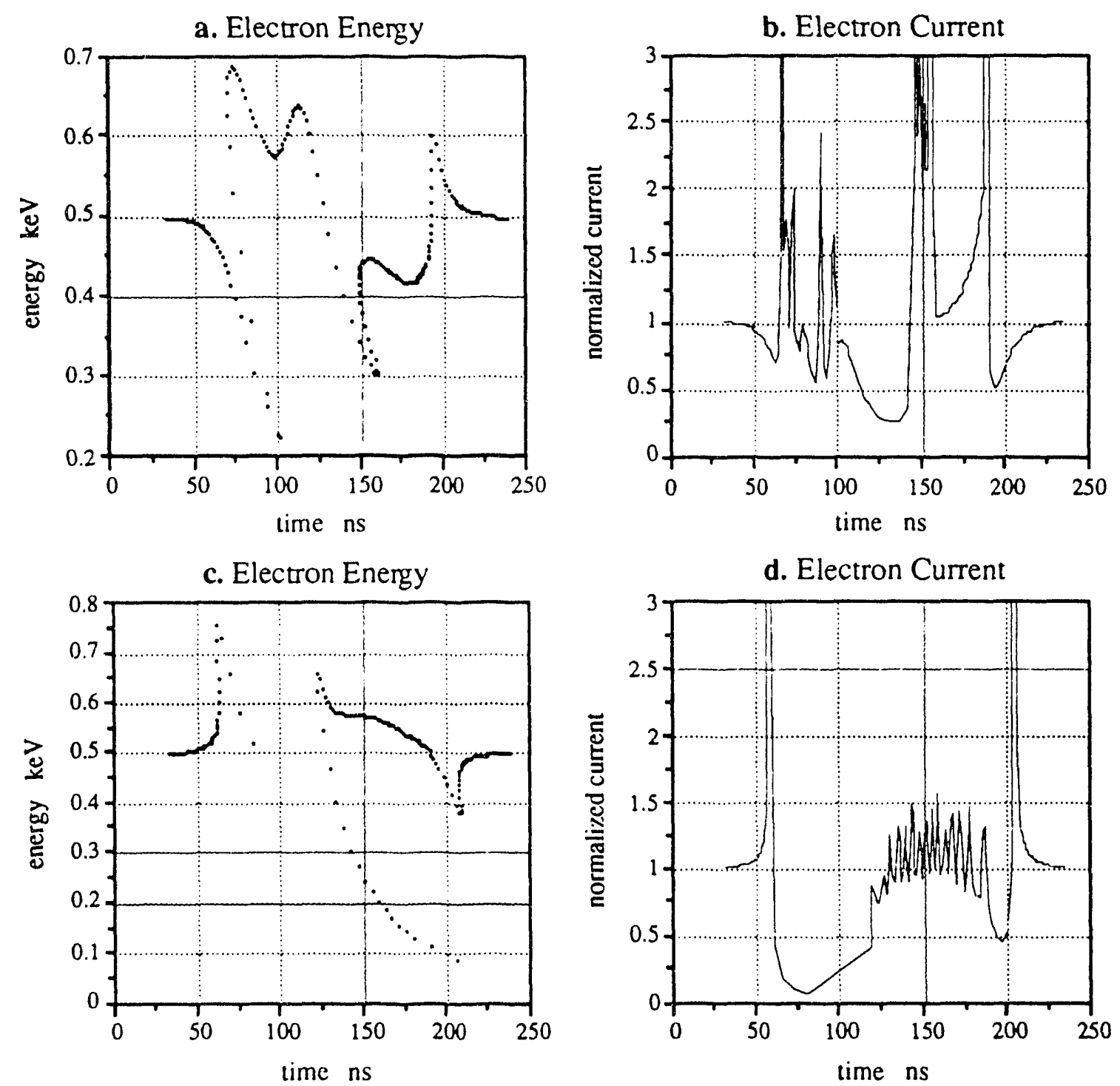

Fig. 6.11 Simulated energy and current collected in the detector of a 500 $\mathrm{eV}$ electron beam passing through the pulser coil region. Parameters used in the ring potential model are 500 volts peak and scale length of $3.8 \mathrm{~cm}$. In a. and $\mathbf{b}$. the polarity is normal and in $\mathbf{c}$. and $\mathbf{d}$. it is reversed. 

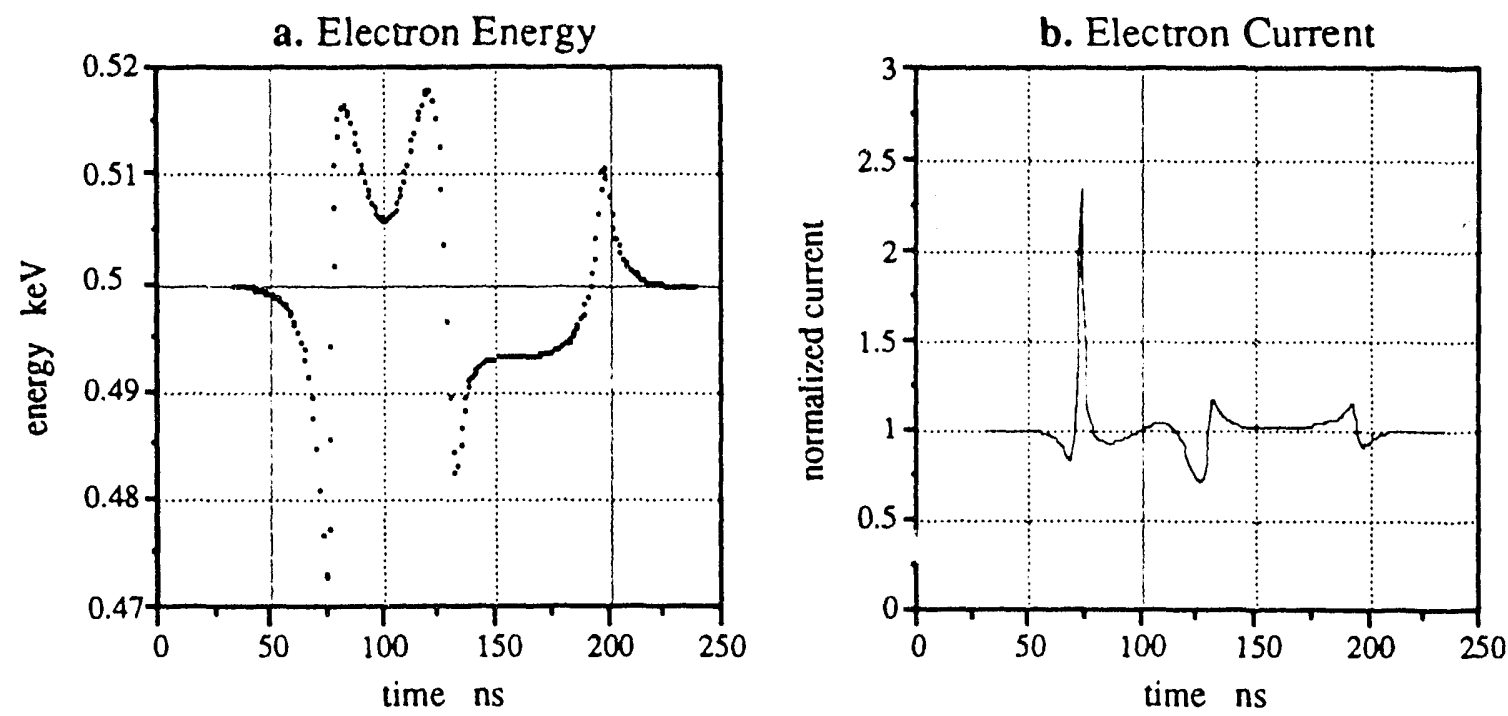

Fig. 6.12 Simulated energy and current signal for the case of a $500 \mathrm{eV}$ electron beam in a weak electrostatic pulser coil potential with a peak of 50 volts. A scale length of $3.8 \mathrm{~cm}$ is used in the ring model of the potential.

The effect of the $\mathrm{L}$ parameter is shown in fig. 6.13. Increasing $\mathrm{L}$ seems to round out the sharpness of the peaks in energy as well as add a little to the magnitude of the variation. Neither parameter seems to affect the period of the oscillations, in other words the energy of the electron train always seems to cross the steady-state energy $(500 \mathrm{eV})$ at the same points in time. But there does appear to be an increase in the length of time during which the current is depressed at the beginning of the fluctuation.

These electron beam current fluctuations can be used to help us develop a better model of the potential shown in fig. 6.7. To do this, an improved understanding of what causes the behavior observed above is necessary. This behavior is understood in the following way. The electron at the bottom of the first dip in the energy corresponds to an electron at the location of the positive peak in the potential when the pulse starts. This location is nominally the axial position of the positive feedthroughs. The electron at the minimum is most attracted to the positive region, so it is pulled back the furthest as it travels to the collector cup. 
The electron at the top of the first peak in energy corresponds to the electron located at the negative potential peak (negative feedthrough) when the pulse starts. This electron feels the full accelerating effect between the two feedthroughs before it comes into the decelerating zone as it leaves the area. This electron gains the most energy, at least in its neighborhood of electrons.
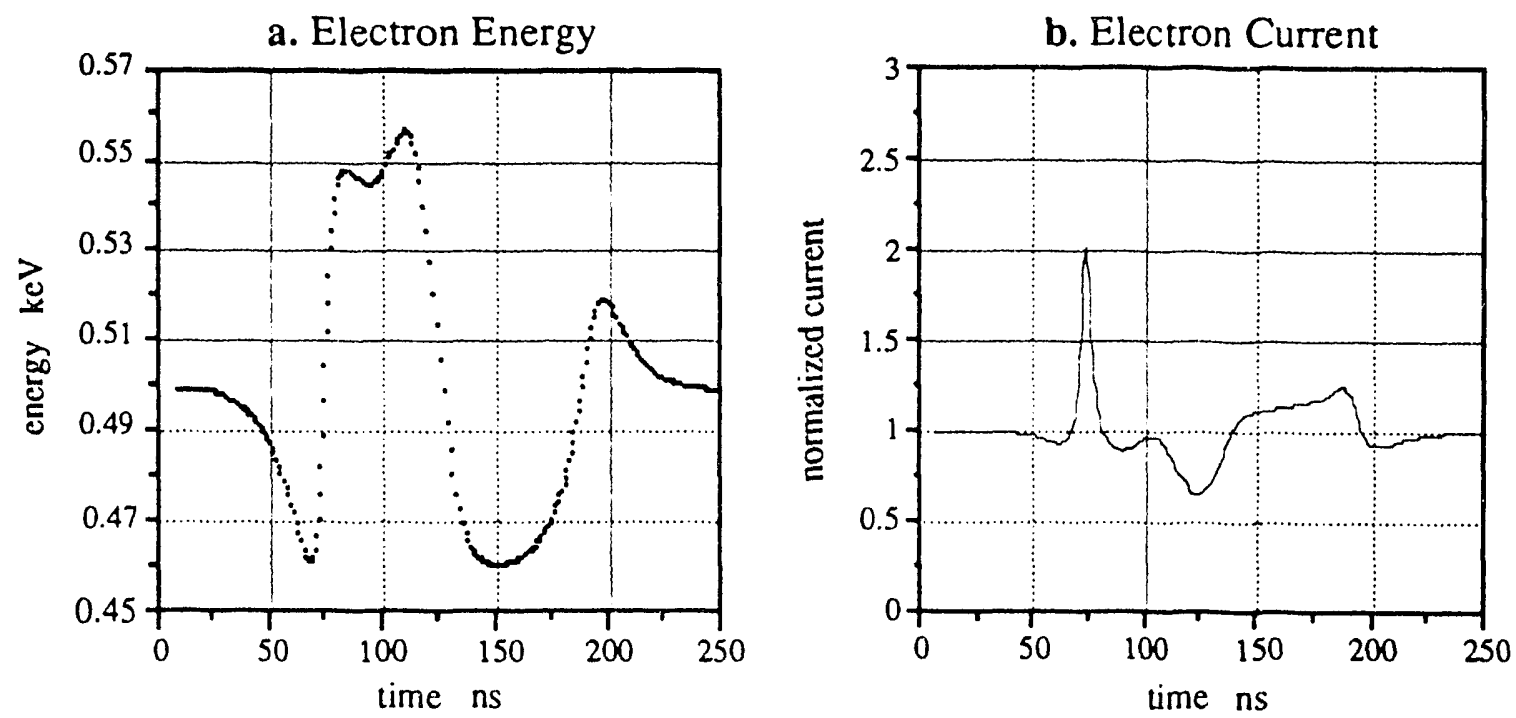

Fig. 6.13 Simulated energy and current signal for the case of a $500 \mathrm{eV}$ electron beam in a weak electrostatic pulser coil potential with a peak of 50 volts. A scale length of $10 \mathrm{~cm}$ is used in the ring model.

The remainder of the energy variation in the electron beam can be explained in terms of the electrons initial position when the pulse starts and the state of the potential when the electrons enter the midplane area. The important part to model is the initial variation, because the beam current later in time is confused by accelerated plasma electrons and a changing ion distribution.

This explanation of the electron beam behavior helps us to understand that the tine of the first beam current depression depends on the spatial extent of the potential from the feedthroughs away from the midplane. All the electrons in this current depression are ones that are pulled back as they are leaving, none of them having passed through the midplane. 
The length of time of the current depression could be shorter, depending on the acceleration of the electrons behind the slow ones and on how long it takes them to catch up. But the time of the current depression will put a lower limit on the length of the spatial extent of this electrostatic potential.

To examine this idea, a potential model is used where the potential gradient at the midplane is constant but the wings of the potential can be extended by varying the parameter L. This model is plotted in fig. 6.9 and is given below.

$$
\begin{array}{cl}
\Phi_{0} \exp \left(\frac{z+3.8}{L}\right) & z<3.8 \mathrm{~cm} \\
\Phi_{0} L & -3.8<z<3.8 \mathrm{~cm} \\
-\Phi_{0} \exp \left(-\frac{z-3.8}{L}\right) & z>3.8 \mathrm{~cm}
\end{array}
$$

A simulation using this potential is shown in fig. $6.14 . \Phi_{0}$ is set to $50 \mathrm{~V}$ and $\mathrm{L}$ is set to $25 \mathrm{~cm}$. The current profile now looks very similar to what was observed (up to the first current spike) and healthy gains in energy are seen, not to different from what was observed. In fig. 6.14.b a current depression lasting for about $80 \mathrm{~ns}$ is seen. In fig. 6.14.c and $d$ the polarity is reversed. The current distribution now resembles fig. 6.8 more. The initial spike is wider than the first simulation, and the current depression after the spike is longer, both of which come closer to the experimental results.

The value for $\Phi_{0}$ is probably lower here than it would be in reality because the average value of $\partial \Phi / \partial x$ over the midplane area is higher than it would be in any realistic model. In fact, for a model used where the potential gradient was infinite just at the midplane, enormous changes in energy were observed for $\Phi_{0}$ as low as $1 \mathrm{~V}$. 

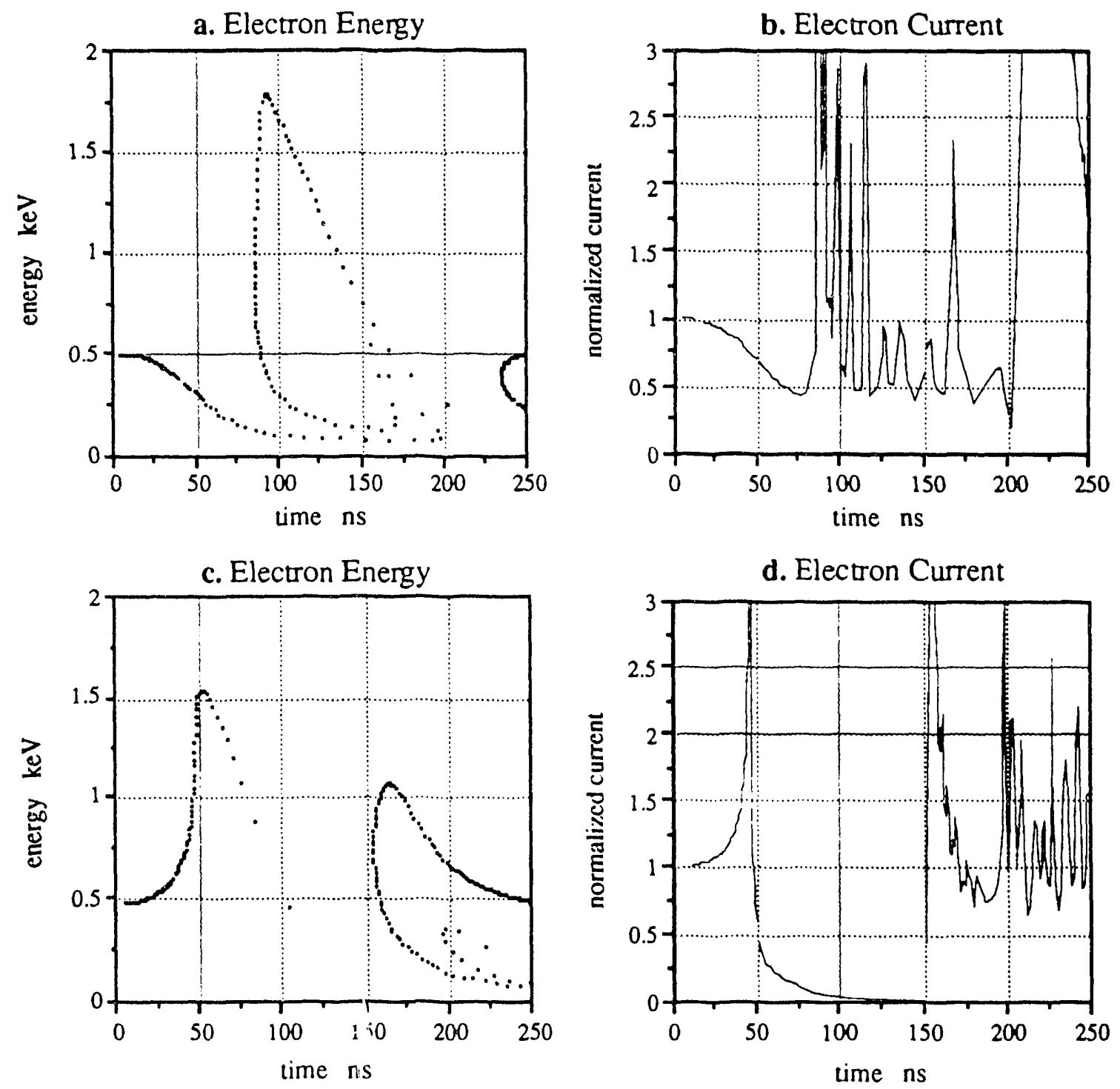

Fig. 6.14 Simulation of a $500 \mathrm{keV}$ electron beam using the potential described in eq. 6.3. Parameters are a 50 volt peak potential and a $25 \mathrm{~cm}$ scale length. In $\mathbf{c}$. and $\mathbf{d}$. the potential polarity is reversed. 


\subsection{End of Phase Two}

At this point, the initial investigation of the experimental design to produce Mirrortron type potentials had come to an end. The electron beam proved sensitive to an axial electric field, even though they were not the fields planned for. The electron beam has pointed out an area for attention in the design of the pulser coil. Due to the inductive impedance of the strap segments, very different potentials formed on each end of the segments. Electrostatic fields formed between these different potential areas. In our particular design, these electric fields had axial components. The best design is to keep the feedthroughs all in the same plane.

Another area for concern was alluded to in reference to fig. 6.2. Even with the interference from the electrostatic fields, the presence of a plasma should have influenced the current fluctuation in some fashion. Apparently though it did not. This suggests that the plasma density was too low to allow a Mirrortron type potential. An attempt to correct this problem was made by increasing the gas flow. The aim was to increase the background gas density and hopefully increase the plasma density.

The gas pressure was increased up to $1.010^{-5}$ torr. At this point the $\mathrm{x}$-ray level was too low to measure. The tack switch always closed shortly after the LF heating stopped. Sometimes the HF heating would stop with the LF or it would continue long after the plasma decayed away. The stopping of the gas flow varied up to $1 \mathrm{sec}$. before the LF stopped. In all cases the electron beam current continued to fluctuate almost unaffected. It now appears that higher power RF sources are necessary. 


\section{Chapter VII Conclusion}

In this project the study of the production of a Mirrortron type potential was begun. It began with the theory of transient potentials resulting from fast rising magnetic gradients in a plasma. The bulk of the work was on the design of the mirror confinement machine and the technology related specifically to potential production and measurement: the pulser coil and the electron beam diagnostic. This study was helpful in revealing areas for concern in the design of a puised magnetic field system.

\subsection{Summary}

\subsubsection{Theory}

One of the first things to be done was a closer analysis of the Mirrortron space potential theory. What started out as a simple non-relativistic analytic expression derived using simplifications was expanded to a more detailed analysis and a relativistic treatment. The detailed analysis indicated that a stronger space potential could be produced for the same pulsed magnetic field than originally thcught. This was 
encouraging. The relativistic analysis shows that even in the extreme relativistic limit the magnitude of the potential approaches the value in the nonrelativistic case when the pulsed magnetic field is about $40 \%$ or more of the original background field. However, when the pulsed field is first applied, the space potential can become negative, especially for the more energetic electron distributions.

An analysis of the assumptions in the derivation of the Mirrortron potential specified certain conditions that must be reproduced in the experiment. The necessity for a slow moving, cold ion background suggested that heavy ions are best suited, however, hydrogen satisfied the purposes of this experiment. The plasma criterion and the necessity of preventing the magnetic pulse from being shielded out of the plasma led to a density range of between $10^{9}$ and $10^{10} \mathrm{~cm}^{-3}$ that was expected to work best. Requirements on the pulsed magnetic field were derived as well. Only a large enough potential to produce measurable effects was required. However, by aiming to produce as strong a potential as possible, a requirement of about 10 gauss per nanosecond was developed for the pulser coil.

\subsubsection{Experiment Design}

One of the first accomplishments was designing and building a magnetic mirror confinement system for a hot electron plasma. A mirror field with a ratio of 1.5 and a midplane strength of 700 gauss was made. Other aspects of the design involved calculations on conductances, pumping speeds, gas flow, decay rates, magnetic field calculations and its effectiveness at adiabatically trapping energetic electrons. The design also included the use of permanent magnets in an octupole configuration for MHD stability. An analysis of this design showed that the plasma column trapped in this field should be very uniform axially at the midplane and drop off quickly near the mirror region. The plasma radial profile will vary azimutinally but is flat for a $5 \mathrm{~cm}$ radius around the axis. In eight radial directions the plasma density will be slightly peaked off axis. 
Part of the experiment design involved creating and heating the plasma to high temperatures using very modest microwave power. Coupling to cavity resonances and maintaining a high $\mathrm{Q}$ factor was crucial to this success. Though the mode analysis was an important start to finding a good operating frequency, eventually an empirical method seemed to work best. The complexity of modes for other than simple cylindrical cavities and the number of modes at higher frequencies made an analysis somewhat impractical. Hence, the importance of tunable sources with a wide range was demonstrated.

A pulser coil system was created for the experiment. Work began with the design of a Blumlein transmission line pulse generator. It was made to hold a $10 \mathrm{kV}$ charge. It was a flat stripline with a one ohm output impedance. Sixteen pulsers were used to drive current in parallel through eight strap coil segments. This strap coil was mounted inside the vacuum chamber with specially designed chokes to prevent the microwave fields from propagating out and reducing the power coupled to the cavity and the plasma.

The Blumlein pulse generators were able to produce a voltage equal to the charge on the line in $20 \mathrm{~ns}$ and the total width of the initial voltage pulse was 60 to $70 \mathrm{~ns}$. The pulser system produced 150 gauss in $70 \mathrm{~ns}$ when a positive and negative $10 \mathrm{kV}$ charge was placed on the pulse generators. This is about 2 gauss per ns or about a factor of five less than what was initially called for. Nevertheless, it was expected that the pulser performance would suffice.

A novel idea for measuring transient, localized space potentials was devised using a monoenergetic electron beam. The electrons passed through regions with time varying electric fields. The resulting bunching effects caused easily detectable current depressions and spikes. The measured current was roughly reproduced in simulations with the help of a simulation program. This program was used with the beam current information to develop a model for the potential field on axis. Though this was done for the unexpected electrostatic fields from the pulser coil, there is no reason that the same method can not be done for the Mirrortron potential. 


\subsubsection{Diagnostics}

The plasma diagnostics used on this experiment were few and simple. It was thought that basic plasma generation would be simple and that the difficult part would come in generating high electron temperatures. A scintillation crystal together with a pulse counting system was used to analyze the $\mathrm{x}$-ray output, and this was used as a basic indication of the electron temperature. High temperatures were easily measured so we were quickly satisfied on this aspect of the operation.

To measure the plasma density, the shift of the cavity resonant frequencies was determined. This was equivalent to a volume averaged density. The actual translation to density was complicated by the necessity of solving an integral equation that depends on the model used for the plasma profile. Uncertainty about other factors such as anisotropy, hot temperature effects on plasma conductivity, and relativistic effects made exact determination of the conversion factor between $\Delta f$ and $n_{0}$ incomplete. It was not done until after the testing in phase one was complete. Had this been done it might have been realized sooner that the plasma density was less than desired. This aspect of plasma operation could have been pursued further, but it was important to proceed to the next phase of the experiment.

Keeping the cavity free of electrical feedthroughs was important to maintain a high $\mathrm{Q}$ factor. One of the implications of this was that some plasma diagnostics, such as Langmuir probes, could not be used. Such probes could not have been used in any event because they would have destroyed the hot electron component. The pulser coil straps were used to some extent for this purpose but the design and shape were not really suited for probing the density, and data interpretation would not have been so simple. Particle collection outside the microwave cavity, on the disc and in the beam collecting cup, was a good relative gauge of the plasma density, but it was not an absolute measure of the density. 
Additional work was done on a diamagnetic loop. The purpose of this diagnostic was to measure the decay of the energy density in the plasma to help optimize the tack switch closing time. No measurements were made with it, however. Much was learned about electronics in an attempt to deal with the noise signal produced by the electromagnets, especially when the noisy arc welder supplies came on line. Ultimately the problem was that the signal was too weak because the energetic part of the plasma was too low in density and/or it decayed too slowly. A few hundred milliseconds was the observed decay time from $x$-ray data.

\subsubsection{Complications}

The design of the pulser coil feedthroughs provided some unexpected electrostatic fields that complicated the electron beam diagnostic. These fields would have masked any fields produced by Mirrortron type effects. However, this electrostatic field provided a good bench test for the electron beam diagnosic.

Positive voltage was applied to the feedthroughs on one side of the midplane and negative voltage was applied to the feedthroughs on the other side. This resulted in a strong electric field across the midplane, even on the axis of the vacuum vessel. The electron beam determined that the electric fields from the feedthrough posts (where the strap segments are anchored) to the grounded vacuum wall were just as important. This is because the potential model that best described the observed current fluctuation is one where the electrostatic potential extends for about 2 to 3 times the pulser coil radius along the axis from the midplane. Also, this model roughly predicted the gain in energy of the beam.

In spite of this effect there should still have been a noticeable difference between a shor without a plasma and a shot with plasma. The Mirrortron potential would have produced an additional current fluctuation. The ideal plasma density would have been enough to shield out the electrostatic potential formed at the feedthroughs near the wall. 
The only significant difference that was observed was that the steady-state electron current was decreased because of collisions and scattering due to the plasma, and also, the signal was a bit noisier, especially later in the shot when plasma particles are accelerated out.

In the first phase it was observed that the $\mathrm{x}$-ray flux increastei when weaker midplane fields were used, i.e. a higher mirror ratio. This suggested higher electron densities were obtained as would be expected, however, this probably would not have led to a higher space potential, as suggested by the theory. Higher mirror ratios only led to smaller $\partial \Phi / \partial B$, a smaller gain in potential per applied magnetic field. Even in the case of the relatively shallow mirror produced by the full strength electromagnet power supplies, and for a $100 \mathrm{keV}$ plasma like ours, $\Phi$ should not be expected to become positive until B increases at least $13 \%$ above its steady-state value, according to fig. 2.14. In a 700 gauss field, this requires 90 gauss, which calls for a minimum charge of $6 \mathrm{kV}$ on the Blumleins. Most shots were taken using a $4 \mathrm{kV}$ charge to protect the pulse generator from premature breakdown while the electrostatic potential problem was investigated. In light of this, the data taken is not inconsistent with the theory describing the potential behavior. Hence, further work is necessary to bear out the Mirrortron concept.

\subsection{Suggestions for Future Work}

Now that the nature of some of the previous problems are understood, the next step is to make corrections. In general, the pulser coil design must take into consideration electrostatic fields that result when the voltage pulses are applied to the feedthroughs. Also steps must be taken to increase the plasma density. The following are suggestions if work is to continue on this particular machine.

The electrostatic potential problem might be alleviated by a Faraday shield. However, a more attractive solution could be applied by reversing the polarity on every other pair of feedthroughs and changing the strap segments from " $Z$ " shapes to " $U$ " 
shapes. Current will still be driven o.e way around the axis and electrostatic fields will still exist between the feedthroughs but these fields will average out to zero on the axis where the electron beam is traveling. This will require a redesign of the pulse generator to allow positive and negative voltage tc alternate on the feedthroughs. This can most easily be done by a redesign of the radial transmission switch line. If tabs are formed in the charge discs that can be slipped past each other then, their top-bottom positions can be reversed every $45^{\circ}$. This will have consequences on the shape of the pulse front as it propagates through these convoluted layers from the tack switch at the center. However, this still may be the best alternative.

Higher plasma density must be maintained. Part of the solution to this problem is the use of higher microwave power. The magnetrons are rated for 300 watts of power but only between 50 and 100 watts were used because the sources were not designed to handle any reflected power and the concern was that the magnetrons might be damaged. In this experiment isolators were used, but more efficient means for handling high reflected power must be used, such as a circulator and dummy load. In addition, better means of transferring the power than through co-ax cables should be used. Direct waveguide connection to the cavity is probably best.

Another improvement in the density could come from better plasma confinement. This might be achieved by moving the permanent magnet octupole closer to the axis. Each permanent magnet row was placed in one long rack that sat on top of the flanges of the vacuum vessel at a distance of $17 \mathrm{~cm}$ from the axis. If the rows were divided into three shorter sections that fit between the flanges, then they could be placed on the vacuum wall at a closer distance of $13 \mathrm{~cm}$. This would move the minimum point in the magnetic field intensity closer to the axis and make a deeper magnetic well for the plasma. The sacrifice would be a discontinuity in the radial fields at the flange locations, but even this can be ameliorated by shimming magnets at these points. 
Concerning the diagnostics, a simple improvement might be the extension of the microwave choke idea to a Langmuir probe. If this can be done without seriously reducing the $\mathrm{Q}$ of the cavity, then it would be useful in measuring the plasma density, even if only data from the plasma edge can be taken. It could also possibly be used to measure quasi-electrostatic fields produced in the cavity during pulser coil operation. Another possible use might be to collect the current in the electron beam diagnostic at a different location, where the bunching effects might not be so piominent.

Turning the Mirrortron concept into a useful machine for accelerating heavy ions is an ambitious project. Significant accomr tishments have been made in the first steps toward achieving this goal. This dissertation has served as an important review of the work that has been done to date so that future work can proceed more efficiently. 


\section{Bibliography}

[1] R F Post, L A Schwager, S R Douglass, et. al., "Studies of the Mirrortron Ion Accelerator Concept and Its Application to Heavy-Ion Drivers," Particle Accelerators 37.38, p.325 (1992)

[2] V. Brady, A. Faltens, et. al., "Heavy-Ion Linear Induction Accelerators as Drivers for Inertial Fusion Power Plants," Fusion Technology, 13, p255 (1988)

[3] D.P.Grubb et al., "Thermal Barrier Production and Identification in a Tandem Mirror," Phys. Rev. Lett., 53, 783 (1984)

[4] R. Gellar, N. Hoffgarten, et. al., "Electric Fields Paralle! to the Magnetic Field in a Laboratory Plasma in a Magnetic Mirror Field," J. Plasma Physics 12, p 467

[5] R.A. Dandl, H.O. Eason, "High-Beta Relativistic Electron Plasmas in Axisymmetric and Non-Axisymmetric Mirrors," LAEA, Proceeding of the 4th Conference on Plasma Physics and Controlled Nuclear Fusion, p607 (1971)

[6] R.F. Post, "The Magnetic Mirror Approach to Fusion," Nuclear Fusion 27 p1681 (1987) 
[7] R.F. Post, "Generation and Control of High Transient Electrical Potentials within a Mirror-Confined Plasma," Phys. Rev. Letters 58 p878 (1987)

[8] L. Spitzer, Physics of Fully lonized Gases, Interscience, New York, p133, (1962)

[9] F. F. Chen, Introduction to Plasma Physics and Controlled Fusion, Plenum Press, New York, p181 (1984)

[10] J.A. Giordmaine, T.C. Wang, "Molecular Beam Formation by Long Parallel Tubes," Jour. of Applied Phys. 31 p463 (1960)

[11] R.F. Post, "The Magnetic Mirror Approach to Fusion," Nuclear Fusion 27 p1611 (1987)

[12] A. Goede, G. J. Brakenhoff, et. al., "Properties of an Extremely Quiescent Plasma Stabilized with Permanent Magnets," Plasma Physics 15 p977 (1973)

[13] L. Spitzer, Physics of Fully lonized Gases, Interscience, New York, p148, (1962)

[14] Kulenkampf, American Institute of Physics Handbook, McGraw-Hil, New York, p7-127, (1972)

[15] S. J. Buchsbaum, L. Mower, et. al., "Interaction Between Cold Plasmas and Guided Electromagnetic Waves," Physics of Fluids, 3 p806 (1960)

[16] J. E. Drummond, Phys. Rev., 110 p293 (1958)

[17] W. W. Hansen, R. F. Post, "On the Measurement of Cavity Impedance," J. Applied Physics, 19 p1059 (1948)

[18] R. Druce, G. Vogtlin, "Subnanosecond Rise Time, Low Impedance Pulse Generator," 4th IEEE Pulsed Power Conference, Albuquerque, New Mexico 

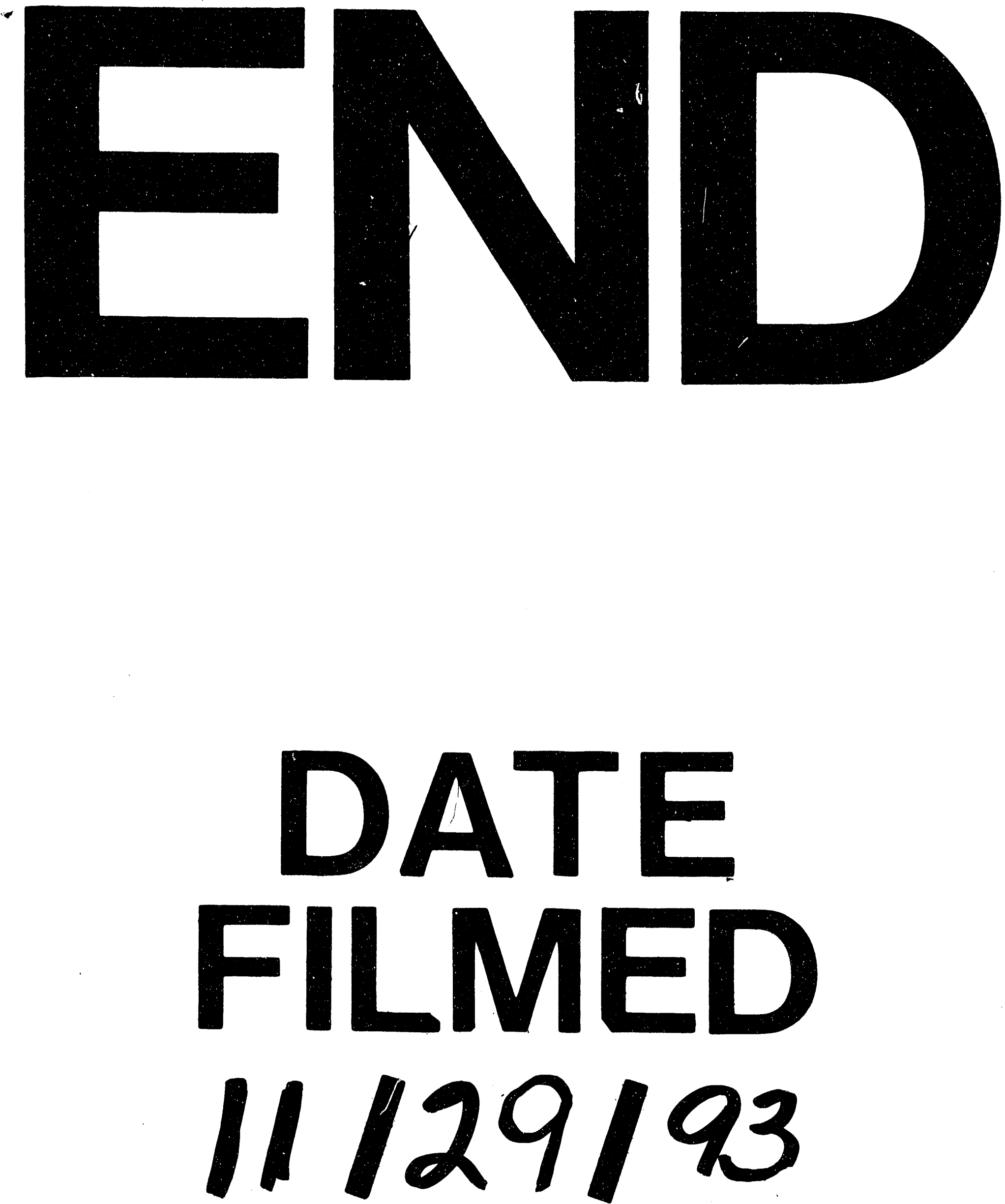

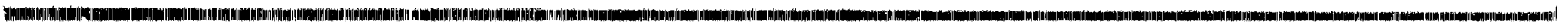


